\title{
One-Pot Synthesis of Benzene-Fused Medium-Ring Ketones: Gold Catalysis-Enabled Enolate Umpolung Reactivity
}

\author{
Zhou Xu ${ }^{a, b}$ Hongyi Chen, ${ }^{a}$ Zhixun Wang ${ }^{a}$ Anguo Ying ${ }^{\mathrm{a}}$ and Liming Zhang ${ }^{a *}$ \\ ${ }^{a}$ Department of Chemistry \& Biochemistry, University of California, Santa Barbara, CA 93106 \\ ${ }^{b}$ Department of Chemistry, Xuzhou Medical College, Xuzhou, Jiangsu,China 221004
}

\section{Supporting Information}

Table of Contents

I. General Information

S2

II. Preparation of Substrates S2-9

III. One-Pot Synthesis of Benzene-Fused Mid-Sized Cyclic Ketones S10-20

IV. Transformation of $\mathbf{3 a}$ to conjugated enone $\mathbf{5} \quad$ S21

V. References S21

VI. NMR spectra $\quad$ S22-S141 


\section{General Information}

Ethyl acetate (ACS grade), hexanes (ACS grade) were purchased from Fisher Scientific and used without further purification. Anhydrous dichloromethane (HPLC grade), 1,2-dichloroethane (HPLC grade) was purified by distillation over calcium hydride. Tetrahydrofuran was distilled over sodium/benzophenone. 1,1,1,3,3,3-hexafluoro2-propanol (HFIP) and 2,2,2-trifluoroethanol were purchased from Oakwood Products, Inc. and used without further purification. Other commercially available reagents were used without further purification. Reactions were monitored by thin layer chromatography (TLC) using Silicycle precoated silica gel plates. Flash column chromatography was performed over Silicycle silica gel (230-400 mesh). ${ }^{1} \mathrm{H}$ NMR and ${ }^{13} \mathrm{C}$ NMR spectra were recorded on Varian $400 \mathrm{MHz}$, $500 \mathrm{MHz}$ and $600 \mathrm{MHz}$ spectrometers using residue solvent peaks as internal standards $\left(\mathrm{CHCl}_{3},{ }^{1} \mathrm{H}: 7.26 \mathrm{ppm} ;{ }^{13} \mathrm{C}\right.$ : $77.00 \mathrm{ppm}) .{ }^{19} \mathrm{~F}$ NMR spectra were recorded on Varian $400 \mathrm{MHz}$ spectrometer calibrated by trifluoroacetic acid peak $\left(\mathrm{CF}_{3} \mathrm{COOH},{ }^{19} \mathrm{~F}:-76.55 \mathrm{ppm}\right)$.Infrared spectra were recorded with a Perkin Elmer FT-IR spectrum 2000 spectrometer and are reported in reciprocal centimeter $\left(\mathrm{cm}^{-1}\right)$. Mass spectra were recorded with Waters micromass ZQ detector using electrospray method (MeOH as solvent) or Waters GCT Premier time-of-flight mass spectrometer with a field ionization (FI) ion source.

\section{Preparation of Substrates}

\section{Hex-5-yn-2-ylbenzene}

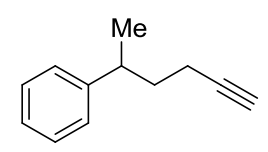

This compound was synthesized according to the literature. ${ }^{[1]}$ The spectra data were identical with those reported. ${ }^{1} \mathrm{H}$ NMR (500 MHz, $\left.\mathrm{CDCl}_{3}\right) \delta 7.33-7.27(\mathrm{~m}, 2 \mathrm{H}), 7.22-7.16(\mathrm{~m}, 3 \mathrm{H}), 2.92-2.82(\mathrm{~m}, 1 \mathrm{H}), 2.15-1.98(\mathrm{~m}, 2 \mathrm{H}), 1.95(\mathrm{t}, J$ $=2.7 \mathrm{~Hz}, 1 \mathrm{H}), 1.84-1.76(\mathrm{~m}, 2 \mathrm{H}), 1.27(\mathrm{~d}, J=7.0 \mathrm{~Hz}, 3 \mathrm{H}) .{ }^{13} \mathrm{C} \mathrm{NMR}\left(125 \mathrm{MHz}, \mathrm{CDCl}_{3}\right) \delta 146.31,128.42,127.02$, $126.14,84.37,68.34,38.71,36.93,21.91,16.67$.

\section{(3-Methoxypent-4-yn-1-yl)benzene}

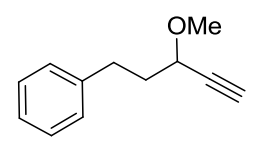

This compound was synthesized according to the literature. ${ }^{[2]}$ The spectra data were identical with those reported. ${ }^{1} \mathrm{H}$ NMR (400 MHz, $\left.\mathrm{CDCl}_{3}\right) \delta 7.29(\mathrm{t}, J=7.5 \mathrm{~Hz}, 2 \mathrm{H}), 7.21(\mathrm{~d}, J=7.3 \mathrm{~Hz}, 3 \mathrm{H}), 3.91(\mathrm{td}, J=6.6,2.0 \mathrm{~Hz}, 1 \mathrm{H}), 3.42(\mathrm{~s}$, $3 \mathrm{H}), 2.79(\mathrm{t}, J=7.8 \mathrm{~Hz}, 2 \mathrm{H}), 2.48(\mathrm{~d}, J=2.0 \mathrm{~Hz}, 1 \mathrm{H}), 2.12-1.95(\mathrm{~m}, 2 \mathrm{H}) .{ }^{13} \mathrm{C}$ NMR $\left(125 \mathrm{MHz}, \mathrm{CDCl}_{3}\right) \delta 141.29$, $128.50,125.95,82.45,74.05,70.14,56.46,37.07,31.26$. 
Preparation of sulfonyl-substituted substrates: the general procedure A:

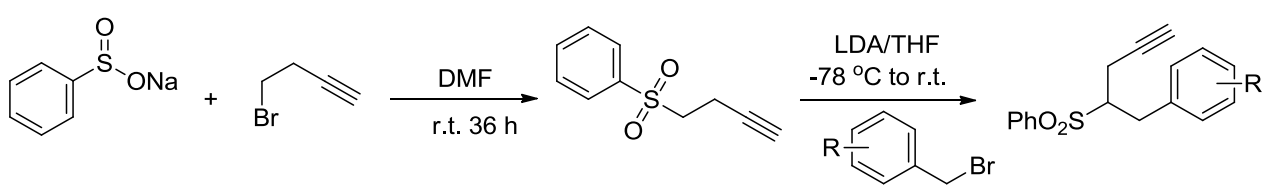

To a solution of sodium benzenesulfinate $(30 \mathrm{mmol}, 4.92 \mathrm{~g}$ ) in DMF $(30 \mathrm{ml})$ was added homopropargyl bromide $(30 \mathrm{mmol}, 3.96 \mathrm{~g})$. The resulting mixture was stirred at room temperature for $36 \mathrm{~h}$. The reaction was quenched with $\mathrm{H}_{2} \mathrm{O}(300 \mathrm{~mL})$ and extracted with $\mathrm{Et}_{2} \mathrm{O}(2 \times 300 \mathrm{~mL})$. The combined organic layers were dried over $\mathrm{Na}_{2} \mathrm{SO}_{4}$, and the solution was concentrated in vacuo to directly afford (but-3-yn-1-ylsulfonyl)benzene ( $2.13 \mathrm{~g}, 78 \%$ yield) as a solid. ${ }^{1} \mathrm{H}$ NMR (500 MHz, $\mathrm{CDCl}_{3}$ ) $\delta$ 7.94-7.89 (m, 2H), 7.70-7.66 (m, 1H), 7.62-7.55 (m, 2H), 3.33-3.25 (m, 2H), 2.67$2.53(\mathrm{~m}, 2 \mathrm{H}), 1.94(\mathrm{t}, J=2.7 \mathrm{~Hz}, 1 \mathrm{H}) .{ }^{13} \mathrm{C} \mathrm{NMR}\left(125 \mathrm{MHz}, \mathrm{CDCl}_{3}\right) \delta 138.44,134.03,129.40,128.24,79.21,70.55$, 54.53, 13.24. IR (neat, $\mathrm{cm}^{-1}$ ): 3289, 3065, 2986, 2933, 2124, 1642, 1586, 1448, 1308, 1151, 1087, 745. ESI ${ }^{+}$calculated for $\left[\mathrm{C}_{10} \mathrm{H}_{10} \mathrm{NaO}_{2} \mathrm{~S}\right]^{+}: 217.03$, found: 217.03 .

To a flame-dried and argon-protected flask were added ${ }^{i} \operatorname{Pr}_{2} \mathrm{NH}$ (4.1 mmol, 2.05 equiv, $\left.0.57 \mathrm{ml}\right)$ and THF (10 ml), and the solution was cooled to $-78^{\circ} \mathrm{C} . n$-BuLi (2.5 M, 2.1 equiv., $4.2 \mathrm{mmol}, 1.7 \mathrm{ml}$ ) was then added dropwise in 15 minutes, and the resulting solution was stirred at the same temperature for $30 \mathrm{~min}$. (But-3-yn-1-ylsulfonyl)benzene ( $2 \mathrm{mmol}, 0.39 \mathrm{~g}$ ) in THF (5 ml) was then added dropwise and the resulting solution was stirred for 30 min before the dropwise addition of benzyl bromide ( $2 \mathrm{mmol}, 1.0$ equiv.) in THF $(5 \mathrm{ml})$ during a $30 \mathrm{~min}$ period. The dry ice-acetone bath was then removed, and the reaction was allowed to warm gradually to room temperature and further stirred for 6 h. The reaction was quenched with water $(25 \mathrm{~mL})$ and extracted with ethyl acetate $(50 \mathrm{~mL})$, the combined organic layers were washed with brine $(50 \mathrm{~mL})$ and dried over $\mathrm{Na}_{2} \mathrm{SO}_{4}$. The solution was concentrated in vacuo, and the resulting crude oil was purified by flash chromatography (hexanes : ethyl acetate $=20: 1$ ), producing the desired alkynes as white solids.

\section{((1-Phenylpent-4-yn-2-yl)sulfonyl)benzene}

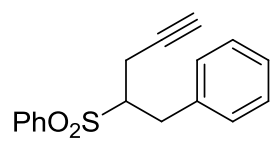

This compound was prepared in $62 \%$ overall yield according to the general procedure $\mathbf{A}$ (eluents: ethyl acetate/hexanes $=1 / 20) .{ }^{1} \mathrm{H} \mathrm{NMR}\left(400 \mathrm{MHz}, \mathrm{CDCl}_{3}\right) \delta 7.94(\mathrm{~d}, J=7.6 \mathrm{~Hz}, 2 \mathrm{H}), 7.66(\mathrm{t}, J=7.5 \mathrm{~Hz}, 1 \mathrm{H}), 7.56(\mathrm{t}, J=7.7 \mathrm{~Hz}, 2 \mathrm{H})$, 7.30-7.14 (m, 5H), 3.36 (ddt, $J=18.8,9.7,4.5 \mathrm{~Hz}, 2 \mathrm{H}), 3.07$ (dd, $J=13.3,9.3 \mathrm{~Hz}, 1 \mathrm{H}), 2.65$ (dt, $J=17.7,3.8 \mathrm{~Hz}$, 1H), 2.56-2.42 (m, 1H), 1.98-1.90 (m, 1H). ${ }^{13} \mathrm{C}$ NMR (125 MHz, $\left.\mathrm{CDCl}_{3}\right) \delta$ 137.63, 136.53, 133.93, 129.17, 129.14, 128.68, 127.03, 78.34, 72.06, 64.59, 32.74, 17.58. IR (neat, $\mathrm{cm}^{-1}$ ): 3283, 3066, 3025, 1640, 1447, 1305, 1146, 1086, 752. $\mathrm{ESI}^{+}$calculated for $\left[\mathrm{C}_{17} \mathrm{H}_{16} \mathrm{NaO}_{2} \mathrm{~S}\right]^{+}: 307.08$, found: 307.09 .

\section{1-Methyl-4-(2-(phenylsulfonyl)pent-4-yn-1-yl)benzene}




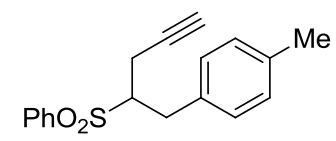

This compound was prepared in $61 \%$ overall yield according to the general procedure $\mathbf{A}$ (eluents: ethyl acetate/hexanes $=1 / 20) .{ }^{1} \mathrm{H}$ NMR $\left(400 \mathrm{MHz}, \mathrm{CDCl}_{3}\right) \delta 7.94(\mathrm{~d}, J=7.6 \mathrm{~Hz}, 2 \mathrm{H}), 7.66(\mathrm{t}, J=7.5 \mathrm{~Hz}, 1 \mathrm{H}), 7.56(\mathrm{t}, J=7.7 \mathrm{~Hz}, 2 \mathrm{H}), 7.07$ (m, 4H), 3.45-3.22 (m, 2H), 3.08-2.92 (m, 1H), 2.64 (ddd, $J=18.0,5.1,2.6 \mathrm{~Hz}, 1 \mathrm{H}), 2.48(\mathrm{ddd}, J=17.9,5.5,2.7 \mathrm{~Hz}$, 1H), 2.30 (s, 3H), 1.93 (t, $J=2.7 \mathrm{~Hz}, 1 \mathrm{H}) .{ }^{13} \mathrm{C} \mathrm{NMR}\left(125 \mathrm{MHz}, \mathrm{CDCl}_{3}\right) \delta 137.69,136.66,133.88,133.35,129.36$, 129.19, 129.11, 129.02, 78.41, 71.99, 64.68, 32.27, 21.01, 17.51. IR (neat, $\left.\mathrm{cm}^{-1}\right): 3281,2920,2854,1510,1447,1306$, 1146, 810, 731. ESI ${ }^{+}$calculated for $\left[\mathrm{C}_{18} \mathrm{H}_{18} \mathrm{NaO}_{2} \mathrm{~S}\right]^{+}: 321.09$, found: 321.09 .

\section{1-Methyl-2-(2-(phenylsulfonyl)pent-4-yn-1-yl)benzene}

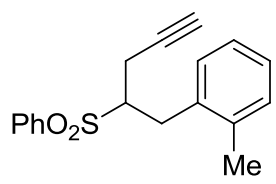

This compound was prepared in $62 \%$ overall yield according to the general procedure $\mathbf{A}$ (eluents: ethyl acetate/hexanes = 1/20). ${ }^{1} \mathrm{H}$ NMR $\left(400 \mathrm{MHz}, \mathrm{CDCl}_{3}\right) \delta 7.97(\mathrm{~d}, J=7.7 \mathrm{~Hz}, 2 \mathrm{H}), 7.67(\mathrm{t}, J=7.5 \mathrm{~Hz}, 1 \mathrm{H}), 7.57$ (t, $\left.J=7.7 \mathrm{~Hz}, 2 \mathrm{H}\right)$, 7.20-7.05 (m, 4H), 3.42 (dd, $J=13.9,3.4 \mathrm{~Hz}, 1 \mathrm{H}), 3.29(\mathrm{dq}, J=9.4,4.2 \mathrm{~Hz}, 1 \mathrm{H}), 3.04(\mathrm{dd}, J=13.9,10.8 \mathrm{~Hz}, 1 \mathrm{H})$, $2.69(\mathrm{dt}, J=17.9,3.5 \mathrm{~Hz}, 1 \mathrm{H}), 2.52(\mathrm{ddd}, J=17.9,5.8,2.8 \mathrm{~Hz}, 1 \mathrm{H}), 2.20(\mathrm{~s}, 3 \mathrm{H}), 1.95(\mathrm{t}, J=2.7 \mathrm{~Hz}, 1 \mathrm{H}) .{ }^{13} \mathrm{C}$ NMR $\left(125 \mathrm{MHz}, \mathrm{CDCl}_{3}\right) \delta 137.58,136.31,134.44,133.89,130.66,129.95,129.07,127.19,126.09,78.37,72.08,62.90$, 30.18, 18.99, 17.23. IR (neat, $\mathrm{cm}^{-1}$ ): 3281, 3069, 2931, 2124, 1631, 1447, 1306, 1147, 1086, 748, 732. ESI ${ }^{+}$calculated for $\left[\mathrm{C}_{18} \mathrm{H}_{18} \mathrm{NaO}_{2} \mathrm{~S}\right]^{+}: 321.09$, found: 321.10 .

\section{1-Methyl-3-(2-(phenylsulfonyl)pent-4-yn-1-yl)benzene}

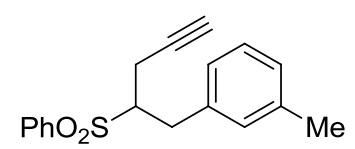

This compound was prepared in 56\% overall yield according to the general procedure A (eluents: ethyl acetate/hexanes = 1/20). ${ }^{1} \mathrm{H}$ NMR (500 MHz, $\left.\mathrm{CDCl}_{3}\right) \delta 7.93(\mathrm{~d}, J=7.9 \mathrm{~Hz}, 2 \mathrm{H}), 7.65(\mathrm{t}, J=7.5 \mathrm{~Hz}, 1 \mathrm{H}), 7.55(\mathrm{t}, J=7.7 \mathrm{~Hz}, 2 \mathrm{H}), 7.15$ $(\mathrm{t}, J=7.5 \mathrm{~Hz}, 1 \mathrm{H}), 7.02(\mathrm{~d}, J=7.7 \mathrm{~Hz}, 1 \mathrm{H}), 6.98-6.96(\mathrm{~m}, 2 \mathrm{H}), 3.34(\mathrm{td}, J=10.2,4.5 \mathrm{~Hz}, 2 \mathrm{H}), 3.02(\mathrm{dd}, J=14.8$, $10.8 \mathrm{~Hz}, 1 \mathrm{H}), 2.64(\mathrm{ddd}, J=17.8,4.9,2.6 \mathrm{~Hz}, 1 \mathrm{H}), 2.52(\mathrm{ddd}, J=17.7,5.5,2.8 \mathrm{~Hz}, 1 \mathrm{H}), 2.29(\mathrm{~s}, 3 \mathrm{H}), 1.94(\mathrm{t}, J=2.7$ $\mathrm{Hz}, 1 \mathrm{H}) .{ }^{13} \mathrm{C}$ NMR $\left(125 \mathrm{MHz}, \mathrm{CDCl}_{3}\right) \delta 138.32,137.71,136.44,133.88,129.83,129.18,129.09,128.56,127.76$, 126.13, 78.43, 71.98, 64.64, 32.69, 21.31, 17.59. IR (neat, $\mathrm{cm}^{-1}$ ): 3282, 2922, 2124, 1608, 1587, 1447, 1306, 1086, 790, 730. ESI ${ }^{+}$calculated for $\left[\mathrm{C}_{18} \mathrm{H}_{18} \mathrm{NaO}_{2} \mathrm{~S}\right]^{+}: 321.09$, found: 321.08 .

\section{1-Chloro-4-(2-(phenylsulfonyl)pent-4-yn-1-yl)benzene}<smiles>C#CCC(Cc1ccc(Cl)cc1)C(=O)Oc1ccccc1</smiles> 
This compound was prepared in $60 \%$ overall yield according to the general procedure A (eluents: ethyl acetate/hexanes $=1 / 20) .{ }^{1} \mathrm{H}$ NMR $\left(500 \mathrm{MHz}, \mathrm{CDCl}_{3}\right) \delta 7.91(\mathrm{dd}, J=7.4,1.6 \mathrm{~Hz}, 2 \mathrm{H}), 7.70-7.64(\mathrm{~m}, 1 \mathrm{H}), 7.59-7.51(\mathrm{~m}, 2 \mathrm{H}), 7.25-$ $7.21(\mathrm{~m}, 2 \mathrm{H}), 7.16-7.11(\mathrm{~m}, 2 \mathrm{H}), 3.38-3.26(\mathrm{~m}, 2 \mathrm{H}), 3.09(\mathrm{dd}, J=13.6,8.6 \mathrm{~Hz}, 1 \mathrm{H}), 2.63(\mathrm{ddd}, J=17.6,5.7,2.7 \mathrm{~Hz}$, 1H), 2.51 (ddd, $J=17.6,5.1,2.8 \mathrm{~Hz}, 1 \mathrm{H}), 1.96(\mathrm{t}, J=2.7 \mathrm{~Hz}, 1 \mathrm{H}) .{ }^{13} \mathrm{C} \mathrm{NMR}\left(125 \mathrm{MHz}, \mathrm{CDCl}_{3}\right) \delta 137.51,135.04$, 134.03, 132.97, 130.57, 129.23, 129.12, 128.81, 78.20, 72.29, 64.38, 32.17, 17.77. IR (neat, $\mathrm{cm}^{-1}$ ): 3300, 3066, 2920, $2850,2120,1493,1447,1306,1147,1086,1016,839,735$. $\mathrm{ESI}^{+}$calculated for $\left[\mathrm{C}_{17} \mathrm{H}_{15} \mathrm{ClNaO}_{2} \mathrm{~S}^{+}: 341.04\right.$, found: 341.03.

\section{Diethyl 2-benzyl-2-(prop-2-yn-1-yl)malonate}

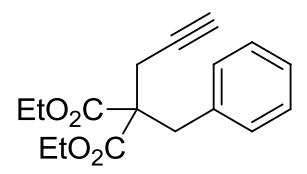

This compound was synthesized according to the literature. ${ }^{[3]}$ The spectra data were identical with those reported. ${ }^{1} \mathrm{H}$ NMR (400 MHz, $\left.\mathrm{CDCl}_{3}\right) \delta 7.29-7.23(\mathrm{~m}, 3 \mathrm{H}), 7.17(\mathrm{~d}, J=7.0 \mathrm{~Hz}, 2 \mathrm{H}), 4.22(\mathrm{~m}, 4 \mathrm{H}), 3.40(\mathrm{~s}, 2 \mathrm{H}), 2.68(\mathrm{~d}, J=2.6$ $\mathrm{Hz}, 2 \mathrm{H}), 2.14(\mathrm{t}, J=2.7 \mathrm{~Hz}, 1 \mathrm{H}), 1.26(\mathrm{t}, J=7.1 \mathrm{~Hz}, 6 \mathrm{H}) .{ }^{13} \mathrm{C} \mathrm{NMR}\left(125 \mathrm{MHz}, \mathrm{CDCl}_{3}\right) \delta 169.61,135.58,129.86$, 128.36, 127.12, 79.36, 72.08, 61.68, 58.10, 37.26, 22.12, 14.01. IR (neat, $\mathrm{cm}^{-1}$ ): 3293, 3033, 2983, 2934, 2123, 1736, $1643,1497,1445,1368,1280,1242,1183,1086,1062,1012,862,704 . \mathrm{ESI}^{+}$calculated for $\left[\mathrm{C}_{17} \mathrm{H}_{20} \mathrm{NaO}_{4}\right]^{+}: 311.13$, found: 311.12 .

\section{$N$-Benzyl-4-methyl-N-(prop-2-yn-1-yl)benzenesulfonamide}

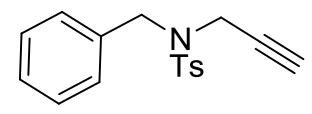

This compound was synthesized according to the literature ${ }^{[3]}$ The spectra data were identical with those reported. ${ }^{1} \mathrm{H}$ NMR $\left(500 \mathrm{MHz}, \mathrm{CDCl}_{3}\right) \delta 7.80(\mathrm{~d}, J=7.7 \mathrm{~Hz}, 2 \mathrm{H}), 7.37-7.29(\mathrm{~m}, 7 \mathrm{H}), 4.36(\mathrm{~s}, 2 \mathrm{H}), 3.95(\mathrm{~d}, J=2.1 \mathrm{~Hz}, 2 \mathrm{H}), 2.45$ (s, 3H), $2.01(\mathrm{~d}, J=1.9 \mathrm{~Hz}, 1 \mathrm{H}) .{ }^{13} \mathrm{C}$ NMR $\left(125 \mathrm{MHz}, \mathrm{CDCl}_{3}\right) \delta 143.58,136.05,134.89,129.49,128.77,128.68$, 128.10, 127.85, 76.29, 74.03, 49.78, 35.52, 21.56. IR (neat, $\mathrm{cm}^{-1}$ ): 3283, 3040, 2923, 2120, 1598, 1496, 1456, 1348, $1161,1095,896,815,768,735$. ESI ${ }^{+}$calculated for $\left[\mathrm{C}_{17} \mathrm{H}_{17} \mathrm{NaO}_{2} \mathrm{~S}\right]^{+}: 322.09$, found: 322.09 .

\section{((But-3-yn-2-yloxy)methyl)benzene}

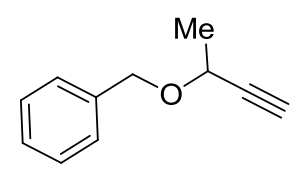

This compound was synthesized according to the literature. ${ }^{[4]}$ The spectra data were identical with those reported. ${ }^{1} \mathrm{H}$ NMR (500 MHz, $\left.\mathrm{CDCl}_{3}\right) \delta$ 7.41-7.32 (m, 4H), 7.32-7.27 (m, 1H), $4.80(\mathrm{~d}, J=11.7 \mathrm{~Hz}, 1 \mathrm{H}), 4.51(\mathrm{~d}, J=11.7 \mathrm{~Hz}$, $1 \mathrm{H}), 4.22(\mathrm{qd}, J=6.6,2.1 \mathrm{~Hz}, 1 \mathrm{H}), 2.47(\mathrm{~d}, J=2.2 \mathrm{~Hz}, 1 \mathrm{H}), 1.48(\mathrm{~d}, J=6.6 \mathrm{~Hz}, 3 \mathrm{H}) .{ }^{13} \mathrm{C} \mathrm{NMR}\left(125 \mathrm{MHz}, \mathrm{CDCl}_{3}\right) \delta$ $137.81,128.39,128.01,127.71,83.71,73.11,70.50,64.20,22.04$. 
The preparation of propargyl ether substrates: the general procedure B:

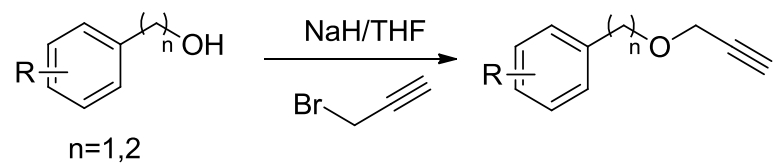

To a solution of the alcohol $(2 \mathrm{mmol})$ in anhydrous THF $(10 \mathrm{ml})$ was added $\mathrm{NaH}$ (1.2 equiv.) in one portion at 0 ${ }^{\circ} \mathrm{C}$. The resulting mixture was stirred for $30 \mathrm{~min}$. A solution of propargyl bromide ( $2 \mathrm{mmol}, 80 \%$ in toluene) was then added dropwise. The reaction was monitored by TLC. Upon completion, the reaction was quenched with water and extracted with ethyl ether $(10 \mathrm{ml} \mathrm{X} 3)$. The combined organic layers were dried over $\mathrm{Na}_{2} \mathrm{SO}_{4}$, filtered and concentrated. The residue was purified by chromatography on silica gel (eluent: hexanes/ethyl acetate) to afford the desired alkynes.

\section{((Prop-2-yn-1-yloxy)methyl)benzene}

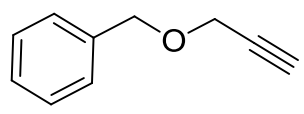

This compound was prepared in 59\% yield according to the general procedure $\mathbf{B}$. The spectra data were identical with those reported. ${ }^{[3]}{ }^{1} \mathrm{H}$ NMR $\left(400 \mathrm{MHz}, \mathrm{CDCl}_{3}\right) \delta$ 7.39-7.27 (m, 5H), $4.62(\mathrm{~s}, 2 \mathrm{H}), 4.18(\mathrm{~d}, J=2.4 \mathrm{~Hz}, 2 \mathrm{H}), 2.47(\mathrm{t}, J=$ $2.5 \mathrm{~Hz}, 1 \mathrm{H}) .{ }^{13} \mathrm{C} \mathrm{NMR}\left(125 \mathrm{MHz}, \mathrm{CDCl}_{3}\right) \delta 137.19,128.40,128.07,127.86,79.60,74.59,71.48,57.01$.

\section{1-Methyl-4-((prop-2-yn-1-yloxy)methyl)benzene}<smiles>C#CCOCc1ccc(C)cc1</smiles>

This compound was prepared in 59\% yield according to the general procedure $\mathbf{B}$. The spectra data were identical with those reported. ${ }^{[5]}{ }^{1} \mathrm{H}$ NMR $\left(500 \mathrm{MHz}, \mathrm{CDCl}_{3}\right) \delta 7.25(\mathrm{~d}, J=7.6 \mathrm{~Hz}, 2 \mathrm{H}), 7.17(\mathrm{~d}, J=7.8 \mathrm{~Hz}, 2 \mathrm{H}), 4.58(\mathrm{~s}, 2 \mathrm{H}), 4.16$ $(\mathrm{d}, J=2.4 \mathrm{~Hz}, 2 \mathrm{H}), 2.46(\mathrm{t}, J=2.3 \mathrm{~Hz}, 1 \mathrm{H}), 2.35(\mathrm{~s}, 3 \mathrm{H}) .{ }^{13} \mathrm{C} \mathrm{NMR}\left(125 \mathrm{MHz}, \mathrm{CDCl}_{3}\right) \delta 137.63,134.17,129.10$, $128.22,79.74,74.46,71.37,56.82,21.16$.

\section{4-((Prop-2-yn-1-yloxy)methyl)phenyl pivalate}<smiles>C#CCOCc1ccc(OP)cc1</smiles>

This compound was prepared in 59\% yield according to the general procedure $\mathbf{B}$ (eluents: ethyl acetate/hexanes = 1/20). ${ }^{1} \mathrm{H}$ NMR $\left(500 \mathrm{MHz}, \mathrm{CDCl}_{3}\right) \delta 7.37(\mathrm{~d}, J=7.8 \mathrm{~Hz}, 2 \mathrm{H}), 7.05(\mathrm{~d}, J=7.8 \mathrm{~Hz}, 2 \mathrm{H}), 4.61(\mathrm{~s}, 2 \mathrm{H}), 4.17(\mathrm{~d}, J=2.4$ $\mathrm{Hz}, 2 \mathrm{H}), 2.47$ (t, $J=2.4 \mathrm{~Hz}, 1 \mathrm{H}), 1.36(\mathrm{~s}, 9 \mathrm{H}) .{ }^{13} \mathrm{C} \mathrm{NMR}\left(126 \mathrm{MHz}, \mathrm{CDCl}_{3}\right) \delta 177.00,150.77,134.63,129.09,121.48$, 79.53, 74.68, 70.86, 57.01, 39.07, 27.12. IR (neat, $\left.\mathrm{cm}^{-1}\right)$ : 2976, 2874, 2117, 1751, 1508, 1279, 1199, 1166, 1120, 896. $\mathrm{ESI}^{+}$calculated for $\left[\mathrm{C}_{15} \mathrm{H}_{18} \mathrm{NaO}_{3}\right]^{+}: 269.13$, found: 269.11 . 


\section{1-((Benzyloxy)methyl)-4-((prop-2-yn-1-yloxy)methyl)benzene}

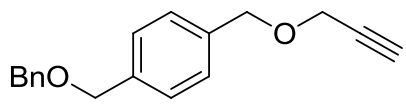

This compound was prepared in $47 \%$ yield according to the general procedure $\mathbf{B}$ (eluents: ethyl acetate/hexanes $=$ 1/50). ${ }^{1} \mathrm{H}$ NMR (500 MHz, $\left.\mathrm{CDCl}_{3}\right) \delta$ 7.41- $7.32(\mathrm{~m}, 8 \mathrm{H}), 7.31-7.27(\mathrm{~m}, l \mathrm{H}), 4.61(\mathrm{~s}, 2 \mathrm{H}), 4.58-4.53(\mathrm{~m}, 4 \mathrm{H}), 4.17$ $(\mathrm{d}, J=2.4 \mathrm{~Hz}, 2 \mathrm{H}), 2.46$ (t, $J=2.4 \mathrm{~Hz}, 1 \mathrm{H}) .{ }^{13} \mathrm{C} \mathrm{NMR}(126 \mathrm{MHz}, \mathrm{CDCl} 3) \delta 138.21,138.03,136.67,128.40,128.22$, 127.89, 127.77, 127.64, 79.63, 74.60, 72.10, 71.82, 71.29, 57.02. IR (neat, $\left.\mathrm{cm}^{-1}\right)$ : 3089, 2857, 2116, 1497, 1454, 1359, $1213,1088,1021,811,739$. ESI ${ }^{+}$calculated for $\left[\mathrm{C}_{18} \mathrm{H}_{18} \mathrm{NaO}_{2}\right]^{+}: 289.13$, found: 289.12 .

\section{2-Bromo-1-methoxy-3-((prop-2-yn-1-yloxy)methyl)benzene}

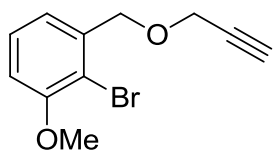

This compound was prepared in $83 \%$ yield according to the general procedure $\mathbf{B}$ (eluents: ethyl acetate/hexanes = 1/50). ${ }^{1} \mathrm{H}$ NMR (500 MHz, $\left.\mathrm{CDCl}_{3}\right) \delta 7.28(\mathrm{t}, \mathrm{J}=8.0 \mathrm{~Hz}, 1 \mathrm{H}), 7.11(\mathrm{~d}, \mathrm{~J}=7.5 \mathrm{~Hz}, 1 \mathrm{H}), 6.85(\mathrm{~d}, \mathrm{~J}=8.1 \mathrm{~Hz}, 1 \mathrm{H}), 4.70$ (s, 2H), $4.26(\mathrm{~d}, \mathrm{~J}=2.3 \mathrm{~Hz}, 2 \mathrm{H}), 3.90$ (s, 3H), $2.48(\mathrm{t}, \mathrm{J}=2.5 \mathrm{~Hz}, 1 \mathrm{H}) .{ }^{13} \mathrm{C}$ NMR (126 MHz, CDCl3) $\delta$ 155.86, 138.56, 127.95, 121.12, 112.20, 111.07, 79.48, 74.81, 71.22, 57.83, 56.38. IR (neat, $\mathrm{cm}^{-1}$ ): 3079, 2941, 2841, 2116, 1574, $1472,1434,1350,1275,1068,1029,775 . \mathrm{ESI}^{+}$calculated for $\left[\mathrm{C}_{11} \mathrm{H}_{11} \mathrm{BrNaO}_{2}\right]^{+}: 276.98,278.98$ found: 276.99, 276.99.

\section{(2-(Prop-2-yn-1-yloxy)ethyl)benzene}

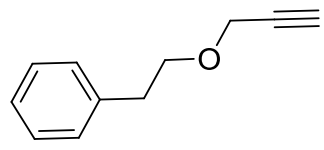

This compound was synthesized according to the general procedure $\mathbf{B}$ (eluents: ethyl acetate/hexanes $=1 / 50$ ). The spectra data were identical with those reported ${ }^{[6]}{ }^{1} \mathrm{H}$ NMR (500 MHz, $\left.\mathrm{CDCl}_{3}\right) \delta$ 7.34-7.27 (m, 2H), 7.25-7.20 (m, $3 \mathrm{H}), 4.17(\mathrm{~d}, J=1.8 \mathrm{~Hz}, 2 \mathrm{H}), 3.76(\mathrm{td}, J=7.6,2.3 \mathrm{~Hz}, 2 \mathrm{H}), 2.93(\mathrm{t}, J=7.0 \mathrm{~Hz}, 2 \mathrm{H}), 2.46-2.37(\mathrm{~m}, 1 \mathrm{H}) .{ }^{13} \mathrm{C} \mathrm{NMR}$ $\left(125 \mathrm{MHz}, \mathrm{CDCl}_{3}\right) \delta 138.59,128.84,128.37,126.27,79.76,74.30,70.84,58.11,36.05$.

\section{1-Methyl-4-(2-(prop-2-yn-1-yloxy)ethyl)benzene}

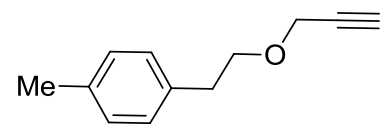

This compound was prepared in $72 \%$ yield according to the general procedure $\mathbf{B}$ (eluents: ethyl acetate/hexanes = 1/50). ${ }^{1} \mathrm{H}$ NMR (500 MHz, $\left.\mathrm{CDCl}_{3}\right) \delta 7.15-7.08(\mathrm{~m}, 4 \mathrm{H}), 4.15(\mathrm{~d}, J=2.4 \mathrm{~Hz}, 2 \mathrm{H}), 3.73(\mathrm{t}, J=7.2 \mathrm{~Hz}, 2 \mathrm{H}), 2.88(\mathrm{t}, J$ $=7.2 \mathrm{~Hz}, 2 \mathrm{H}), 2.42(\mathrm{t}, J=2.4 \mathrm{~Hz}, 1 \mathrm{H}), 2.32(\mathrm{~s}, 3 \mathrm{H}) .{ }^{13} \mathrm{C} \mathrm{NMR}\left(125 \mathrm{MHz}, \mathrm{CDCl}_{3}\right) \delta 135.77,135.46,129.07,128.72$, 79.81, 74.27, 71.01, 58.10, 35.60, 21.01. IR (neat, $\mathrm{cm}^{-1}$ ): 3293, 3018, 2927, 2862, 2120, 1643, 1516, 1442, 1357, 1099, 810. $\mathrm{ESI}^{+}$calculated for $\left[\mathrm{C}_{12} \mathrm{H}_{14} \mathrm{NaO}+\mathrm{MeOH}\right]^{+}: 229.12$, found: 229.08 . 


\section{1,3-Dimethyl-5-(2-(prop-2-yn-1-yloxy)ethyl)benzene}

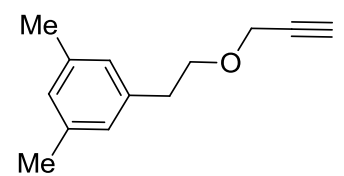

This compound was prepared in $60 \%$ yield according to the general procedure $\mathbf{B}$ (eluents: ethyl acetate/hexanes = 1/50). ${ }^{1} \mathrm{H}$ NMR (500 MHz, $\left.\mathrm{CDCl}_{3}\right) \delta 6.85(\mathrm{~m}, 3 \mathrm{H}), 4.16(\mathrm{~d}, J=2.4 \mathrm{~Hz}, 2 \mathrm{H}), 3.73(\mathrm{t}, J=7.3 \mathrm{~Hz}, 2 \mathrm{H}), 2.85(\mathrm{t}, J=7.3$ $\mathrm{Hz}, 2 \mathrm{H}), 2.42$ (t, $J=2.4 \mathrm{~Hz}, 1 \mathrm{H}), 2.29$ (s, 6H). ${ }^{13} \mathrm{C} \mathrm{NMR}\left(125 \mathrm{MHz}, \mathrm{CDCl}_{3}\right) \delta 138.36,137.86,127.95,126.66,79.84$, 74.25, 70.99, 58.10, 35.88, 21.24. IR (neat, $\mathrm{cm}^{-1}$ ): 3292, 3016, 2919, 2882, 2117, 1607, 1475, 1357, 1099, 847. GCMS: $m / z=188\left[\mathrm{M}^{+}\right], 159,119,103,91$.

\section{1-Methoxy-3-(2-(prop-2-yn-1-yloxy)ethyl)benzene}

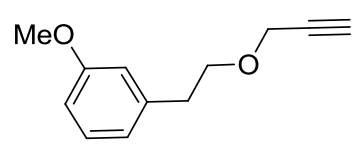

This compound was prepared in $94 \%$ yield according to the general procedure $\mathbf{B}$ (eluents: ethyl acetate/hexanes = 1/50). ${ }^{1} \mathrm{H}$ NMR $\left(500 \mathrm{MHz}, \mathrm{CDCl}_{3}\right) \delta 7.21(\mathrm{t}, J=7.8 \mathrm{~Hz}, 1 \mathrm{H}), 6.83(\mathrm{dt}, J=7.5,1.2 \mathrm{~Hz}, 1 \mathrm{H}), 6.80(\mathrm{t}, J=2.1 \mathrm{~Hz}, 1 \mathrm{H})$, $6.77(\mathrm{ddd}, J=8.2,2.7,0.9 \mathrm{~Hz}, 1 \mathrm{H}), 4.16(\mathrm{~d}, J=2.4 \mathrm{~Hz}, 2 \mathrm{H}), 3.80(\mathrm{~s}, 3 \mathrm{H}), 3.76(\mathrm{t}, J=7.1 \mathrm{~Hz}, 2 \mathrm{H}), 2.90(\mathrm{t}, J=7.1 \mathrm{~Hz}$, 2H), $2.42(\mathrm{t}, J=2.4 \mathrm{~Hz}, 1 \mathrm{H}) .{ }^{13} \mathrm{C} \mathrm{NMR}\left(125 \mathrm{MHz}, \mathrm{CDCl}_{3}\right) \delta 159.62,140.20,129.33,121.21,114.62,111.61,79.75$, 74.32, 70.72, 58.11, 55.13, 36.06. IR (neat, $\mathrm{cm}^{-1}$ ): 3288, 2943, 2861, 2120, 1603, 1585, 1490, 1455, 1260, 1153, 1098, 1042, 779. $\mathrm{ESI}^{+}$calculated for $\left[\mathrm{C}_{12} \mathrm{H}_{14} \mathrm{NaO}_{2}+\mathrm{MeOH}\right]^{+}: 245.12$, found: 245.08 .

\section{1-Methyl-2-(2-(prop-2-yn-1-yloxy)ethyl)benzene}

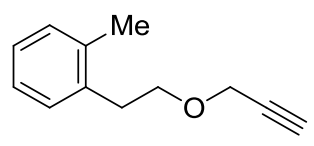

This compound was prepared in $72 \%$ yield according to the general procedure $\mathbf{B}$ (eluents: ethyl acetate/hexanes = 1/50). ${ }^{1} \mathrm{H}$ NMR (500 MHz, $\left.\mathrm{CDCl}_{3}\right) \delta 7.21-7.10(\mathrm{~m}, 4 \mathrm{H}), 4.18(\mathrm{~d}, J=2.3 \mathrm{~Hz}, 2 \mathrm{H}), 3.73(\mathrm{t}, J=7.5 \mathrm{~Hz}, 2 \mathrm{H}), 2.94(\mathrm{t}, J$ $=7.5 \mathrm{~Hz}, 2 \mathrm{H}), 2.43(\mathrm{t}, J=2.4 \mathrm{~Hz}, 1 \mathrm{H}), 2.35(\mathrm{~s}, 3 \mathrm{H}) .{ }^{13} \mathrm{C} \mathrm{NMR}\left(125 \mathrm{MHz}, \mathrm{CDCl}_{3}\right) \delta 136.53,136.35,130.19,129.32$, 126.44, 125.97, 79.76, 74.30, 69.87, 58.09, 33.29, 19.39. IR (neat, $\mathrm{cm}^{-1}$ ): 3293, 2956, 2863, 2118, 1493, 1459, 1357, 1096, 745. ESI ${ }^{+}$calculated for $\left[\mathrm{C}_{12} \mathrm{H}_{14} \mathrm{NaO}\right]^{+}: 197.09$, found: 197.09 .

\section{1-(2-(Prop-2-yn-1-yloxy)ethyl)naphthalene}

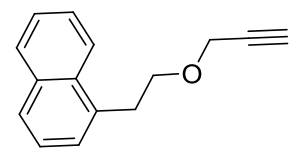

This compound was prepared in $84 \%$ yield according to the general procedure $\mathbf{B}$ (eluents: ethyl acetate/hexanes = 1/50). ${ }^{1} \mathrm{H}$ NMR (500 MHz, $\left.\mathrm{CDCl}_{3}\right) \delta 8.08(\mathrm{~d}, J=8.4 \mathrm{~Hz}, 1 \mathrm{H}), 7.87(\mathrm{dd}, J=8.0,1.4 \mathrm{~Hz}, 1 \mathrm{H}), 7.75(\mathrm{dd}, J=7.6,1.8 \mathrm{~Hz}$, 1H), 7.55-7.47 (m, 2H), 7.44-7.38 (m, 2H), $4.20(\mathrm{~d}, J=2.5 \mathrm{~Hz}, 2 \mathrm{H}), 3.90(\mathrm{t}, J=7.5 \mathrm{~Hz}, 2 \mathrm{H}), 3.42(\mathrm{t}, J=7.4 \mathrm{~Hz}, 2 \mathrm{H})$, 
$2.44(\mathrm{t}, J=2.4 \mathrm{~Hz}, 1 \mathrm{H}) .{ }^{13} \mathrm{C}$ NMR $\left(125 \mathrm{MHz}, \mathrm{CDCl}_{3}\right) \delta 134.42,133.83,132.05,128.77,127.12,126.78,125.94$, 125.50, 125.49, 123.59, 79.75, 74.39, 70.25, 58.16, 33.08. IR (neat, $\mathrm{cm}^{-1}$ ): 3292, 3048, 2947, 2862, 2117, 1597, 1511, $1397,1357,1097,798,778 . \mathrm{ESI}^{+}$calculated for $\left[\mathrm{C}_{15} \mathrm{H}_{14} \mathrm{NaO}+\mathrm{MeOH}\right]^{+}: 265.12$, found: 265.11 .

2-(2-(Prop-2-yn-1-yloxy)ethyl)naphthalene

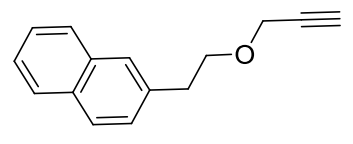

This compound was prepared in $84 \%$ yield according to the general procedure $\mathbf{B}$ (eluents: ethyl acetate/hexanes = 1/50). ${ }^{1} \mathrm{H}$ NMR (500 MHz, $\mathrm{CDCl}_{3}$ ) $\delta 7.84-7.76(\mathrm{~m}, 3 \mathrm{H}), 7.69$ (s, $\left.1 \mathrm{H}\right), 7.44(\mathrm{pd}, J=6.9,1.5 \mathrm{~Hz}, 2 \mathrm{H}), 7.38$ (dd, $J=8.4$, $1.8 \mathrm{~Hz}, 1 \mathrm{H}), 4.18(\mathrm{~d}, J=2.4 \mathrm{~Hz}, 2 \mathrm{H}), 3.85(\mathrm{t}, J=7.1 \mathrm{~Hz}, 2 \mathrm{H}), 3.09(\mathrm{t}, J=7.1 \mathrm{~Hz}, 2 \mathrm{H}), 2.43(\mathrm{t}, J=2.4 \mathrm{~Hz}, 1 \mathrm{H}) .{ }^{13} \mathrm{C}$ NMR $\left(125 \mathrm{MHz}, \mathrm{CDCl}_{3}\right) \delta 136.17,133.56,132.19,127.92,127.59,127.48,127.43,127.14,125.92,125.31,79.76$, 74.36, 70.76, 58.18, 36.21. IR (neat, $\mathrm{cm}^{-1}$ ): 3291, 3054, 2862, 2949, 2120, 1601, 1508, 1356, 1271, 1098, 856, 819, 748. $\mathrm{ESI}^{+}$calculated for $\left[\mathrm{C}_{15} \mathrm{H}_{14} \mathrm{NaO}\right]^{+}: 233.09$, found: 233.09 .

Synthesis of 4-methyl-N-phenethyl-N-(prop-2-yn-1-yl)benzenesulfonamide:

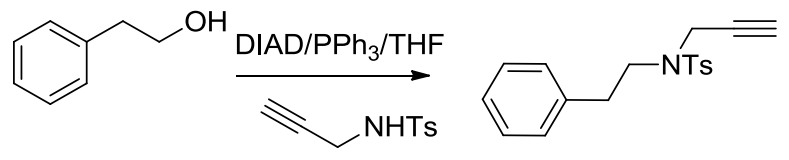

To a solution of 2-phenylethanol (4 mmol) in THF (40 ml) were added $\mathrm{PPh}_{3}(6 \mathrm{mmol}, 1.57 \mathrm{~g})$, 4-methyl- $\mathrm{N}$-(prop-2yn-1-yl)benzenesulfonamide ( $6 \mathrm{mmol}$ ), and diethyl azodicarboxylate (DIAD) (945 uL, $6 \mathrm{mmol}$ ). After being stirred for $40 \mathrm{~h}$ at rt, the reaction was concentrated in vacuo. The crude residue was purified by silica gel flash column chromatography (eluents: ethyl acetate/hexanes $=1 / 5)$ to afford the desired alkyne in $72 \%$ yield. ${ }^{1} \mathrm{H} \mathrm{NMR}(500 \mathrm{MHz}$, $\left.\mathrm{CDCl}_{3}\right) \delta 7.71(\mathrm{~d}, J=8.3 \mathrm{~Hz}, 2 \mathrm{H}), 7.33-7.25(\mathrm{~m}, 4 \mathrm{H}), 7.25-7.18(\mathrm{~m}, 3 \mathrm{H}), 4.10(\mathrm{~d}, J=2.5 \mathrm{~Hz}, 2 \mathrm{H}), 3.47-3.38(\mathrm{~m}$, 2H), 2.94-2.85 (m, 2H), $2.41(\mathrm{~s}, 3 \mathrm{H}), 2.06(\mathrm{t}, J=2.5 \mathrm{~Hz}, 1 \mathrm{H}) .{ }^{13} \mathrm{C} \mathrm{NMR}\left(125 \mathrm{MHz}, \mathrm{CDCl}_{3}\right) \delta 143.47,138.19,135.89$, 129.46, 128.78, 128.57, 127.64, 126.57, 76.70, 73.73, 47.97, 36.85, 34.75, 21.49. IR (neat, $\mathrm{cm}^{-1}$ ): 3282, 3029, 2926, $2872,2120,1598,1497,1455,1348,1160,1097,991,902,815,756 . \mathrm{ESI}^{+}$calculated for $\left[\mathrm{C}_{18} \mathrm{H}_{19} \mathrm{NaO}_{2} \mathrm{~S}\right]^{+}: 336.10$, found: 336.09 .

\section{(Pent-4-yn-1-yloxy)benzene}

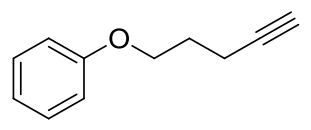

To a mixture of phenol (0.94 g, $10 \mathrm{mmol}), \mathrm{PPh}_{3}(2.62 \mathrm{~g}, 10 \mathrm{mmol})$, and 4-pentyn-1-ol (1.01g, $\left.12 \mathrm{mmol}\right)$ in THF (25 $\mathrm{mL}$ ) was added DEAD $(10 \mathrm{mmol})$ at $0{ }^{\circ} \mathrm{C}$. The mixture was stirred at reflux for $6 \mathrm{~h}$ and cooled to room temperature. After THF was removed under reduced pressure, the residue was chromatographed on silica gel (eluents : hexanes) to afford (pent-4-yn-1-yloxy)benzene (1.34 g, 84\%). ${ }^{1} \mathrm{H}$ NMR (500 MHz, $\left.\mathrm{CDCl}_{3}\right) \delta 7.29$ (t, J = 7.5 Hz, 2H), $6.98-6.89$ $(\mathrm{m}, 3 \mathrm{H}), 4.08(\mathrm{t}, \mathrm{J}=6.1 \mathrm{~Hz}, 2 \mathrm{H}), 2.42(\mathrm{td}, \mathrm{J}=7.0,2.7 \mathrm{~Hz}, 2 \mathrm{H}), 2.05-1.96(\mathrm{~m}, 3 \mathrm{H}) .{ }^{13} \mathrm{C} \mathrm{NMR}(126 \mathrm{MHz}, \mathrm{CDCl} 3) \delta$ 
158.86, 129.43, 120.70, 114.51, 83.51, 68.82, 66.01, 28.22, 15.20. IR (neat, $\mathrm{cm}^{-1}$ ): 3042, 2942, 2877, 2119, 1600, $1498,1471,1246,1052,755$. $\mathrm{ESI}^{+}$calculated for $\left[\mathrm{C}_{11} \mathrm{H}_{12} \mathrm{NaO}^{+}: 183.08\right.$ found: 183.06 .

\section{One-Pot Synthesis of Benzene-Fused Medium-Ring Ketones.}

\section{(1) A representative procedure for the synthesis of benzene-fused 7-membered ketones}

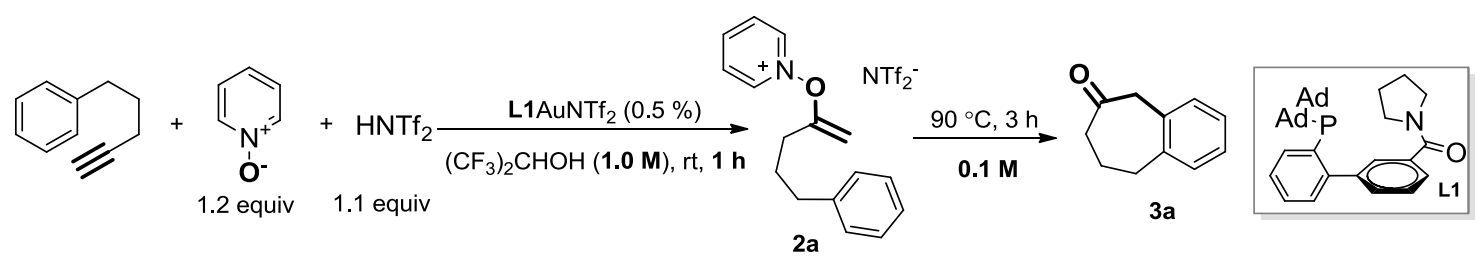

Pyridine $\mathrm{N}$-oxide and $\mathrm{Tf}_{2} \mathrm{NH}$ were premixed in a molar ratio of 1.2/1.1 which was then stored in a vial and used for reaction directly.

$\mathrm{L}_{1} \mathrm{AuNTf}_{2}(1.5 \mathrm{mg}, 0.005$ equiv.) was added into a mixture of pent-4-yn-1-ylbenzene ( $43.8 \mathrm{mg}, 0.3 \mathrm{mmol}$ ), the above premixed salt $(126.9 \mathrm{mg}, 1.2$ equiv. in the $N$-oxide) and HFIP $(0.3 \mathrm{~mL})$ in a vial at room temperature. The reaction mixture was then stirred at room temperature, and the progress of the reaction was monitored by TLC (hexanes/ethyl acetate $=10 / 1)$. After the reaction was completed, the reaction mixture was diluted with HFIP (2.7 ml) and the reaction vial was sealed and heated to $90{ }^{\circ} \mathrm{C}$. The reaction was monitored by TLC (DCM/MeOH $\left.=10 / 1\right)$. Upon completion, the reaction mixture was concentrated, and the resulting residue was purified by flash chromatography on silica gel (eluent: hexanes/ethyl acetate $=10 / 1$ ) to afford the desired product $\mathbf{3 a}$.

\section{1-((5-Phenylpent-1-en-2-yl)oxy)pyridinium triflimide (2a)}

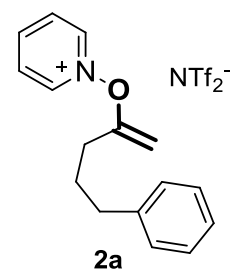

2a can be isolated with flash chromatography on silica gel (eluent: hexanes/ethyl acetate 10/1) with $88 \%$ isolated yield. ${ }^{1} \mathrm{H}$ NMR $\left(500 \mathrm{MHz}, \mathrm{CDCl}_{3}\right) \delta 8.77-8.69(\mathrm{~m}, 2 \mathrm{H}), 8.62(\mathrm{tt}, J=8.2,1.5 \mathrm{~Hz}, 1 \mathrm{H}), 8.27-8.16(\mathrm{~m}, 2 \mathrm{H}), 7.30$ (dd, $J$ $=8.6,6.4 \mathrm{~Hz}, 2 \mathrm{H}), 7.24-7.17(\mathrm{~m}, 3 \mathrm{H}), 4.54(\mathrm{~d}, J=5.6 \mathrm{~Hz}, 1 \mathrm{H}), 3.73(\mathrm{~d}, J=5.5 \mathrm{~Hz}, 1 \mathrm{H}), 2.73(\mathrm{t}, J=7.4 \mathrm{~Hz}, 2 \mathrm{H})$, 2.48-2.35 (m, 2H), 1.99 (p, $J=7.6 \mathrm{~Hz}, 2 \mathrm{H}) .{ }^{13} \mathrm{C}$ NMR (125 MHz, $\left.\mathrm{CDCl}_{3}\right) \delta 165.62,146.73,141.90,140.97,130.31$, 128.56, 126.20, 121.03, 118.48, 89.70, 34.83, 30.49, 27.83. $\mathrm{F}^{19} \mathrm{NMR}\left(\mathrm{CDCl}_{3}, 376 \mathrm{MHz}\right) \delta:-77.66$. IR (neat, $\left.\mathrm{cm}^{-1}\right)$ : $3058,3029,2941,2876,1715,1492,1453,1273,1125,1053,759$. ESI ${ }^{+}$calculated for $\left[\mathrm{C}_{16} \mathrm{H}_{18} \mathrm{NO}\right]^{+}: 240.14$, found: 240.14 . 


\section{8,9-Dihydro-5H-benzo[7]annulen-6(7H)-one (3a)}

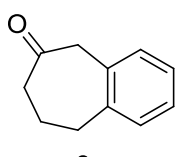

$3 a$

Compound 3a was prepared in $70 \%$ yield according to the above representative procedure (eluents: ethyl acetate/hexanes = 1/10). ${ }^{1} \mathrm{H}$ NMR $\left(500 \mathrm{MHz}, \mathrm{CDCl}_{3}\right) \delta 7.23-7.13(\mathrm{~m}, 4 \mathrm{H}), 3.73(\mathrm{~s}, 2 \mathrm{H}), 3.02-2.86(\mathrm{~m}, 2 \mathrm{H}), 2.57(\mathrm{t}$, $J=6.9 \mathrm{~Hz}, 2 \mathrm{H}), 2.04-1.96(\mathrm{~m}, 2 \mathrm{H}) .{ }^{13} \mathrm{C} \mathrm{NMR}\left(125 \mathrm{MHz}, \mathrm{CDCl}_{3}\right) \delta 208.70,140.42,133.56,129.54,129.18,127.54$, 127.09, 50.14, 43.64, 32.98, 26.24. IR (neat, $\mathrm{cm}^{-1}$ ): 3022, 2933, 2863, 1708, 1491, 1451, 1274, 1222, 949, 755. ESI calculated for $\left[\mathrm{C}_{11} \mathrm{H}_{13} \mathrm{O}\right]^{+}: 161.10$, found: 161.08 .

\section{9-Methyl-8,9-dihydro-5H-benzo[7]annulen-6(7H)-one $(3 \mathrm{~b})$}

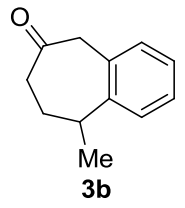

Compound 3b was prepared in $77 \%$ yield according to the above representative procedure (eluents: ethyl acetate/hexanes = 1/10). ${ }^{1} \mathrm{H}$ NMR $\left(500 \mathrm{MHz}, \mathrm{CDCl}_{3}\right) \delta$ 7.33-7.27 (m, 2H), $7.20(\mathrm{td}, J=6.9,2.3 \mathrm{~Hz}, 1 \mathrm{H}), 7.13(\mathrm{~d}, J=$ $7.4 \mathrm{~Hz}, 1 \mathrm{H}), 3.88(\mathrm{~d}, J=17.5 \mathrm{~Hz}, 1 \mathrm{H}), 3.54(\mathrm{~d}, J=17.6 \mathrm{~Hz}, 1 \mathrm{H}), 3.18(\mathrm{ddd}, J=12.1,7.0,5.4 \mathrm{~Hz}, 1 \mathrm{H}), 2.51(\mathrm{ddd}, J=$ 12.5, 10.0, 7.2 Hz, 1H), 2.36 (ddd, $J=12.5,6.2,4.5 \mathrm{~Hz}, 1 \mathrm{H}), 2.12$ (ddt, $J=13.3,11.2,5.8 \mathrm{~Hz}, 1 \mathrm{H}$ ), 1.65-1.53 (m, $1 \mathrm{H}), 1.42(\mathrm{~d}, J=6.8 \mathrm{~Hz}, 3 \mathrm{H}) .{ }^{13} \mathrm{C}$ NMR $\left(125 \mathrm{MHz}, \mathrm{CDCl}_{3}\right) \delta 210.28,143.05,134.06,129.54,127.71,126.61,125.17$, 49.53, 41.21, 34.44, 33.96, 19.39. IR (neat, $\mathrm{cm}^{-1}$ ): 2963, 2927, 2876, 1706, 1452, 1288, 1280, 1256, 761. ESI calculated for $\left[\mathrm{C}_{12} \mathrm{H}_{15} \mathrm{O}\right]^{+}: 175.11$, found: 175.09 .

7-Methoxy-8,9-dihydro-5H-benzo[7]annulen-6(7H)-one (3c)

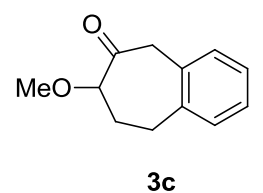

Compound 3c was prepared in $67 \%$ yield according to the above representative procedure (eluents: ethyl acetate/hexanes = 1/10). ${ }^{1} \mathrm{H}$ NMR $\left(500 \mathrm{MHz}, \mathrm{CDCl}_{3}\right) \delta$ 7.20-7.18 (m, 3H), 7.16-7.12 (m, 1H), $4.06(\mathrm{~d}, J=13.5 \mathrm{~Hz}$, 1H), 3.90 (ddd, $J=7.9,5.2,0.9 \mathrm{~Hz}, 1 \mathrm{H}$ ), 3.49 (dd, $J=13.5,0.9 \mathrm{~Hz}, 1 \mathrm{H}$ ), 3.38 (s, 3H), 3.15 (ddd, $J=14.3,11.1,3.0$ $\mathrm{Hz}, 1 \mathrm{H}), 2.78(\mathrm{ddd}, J=14.6,7.3,3.0 \mathrm{~Hz}, 1 \mathrm{H}), 2.14(\mathrm{tdd}, J=11.0,5.2,2.6 \mathrm{~Hz}, 1 \mathrm{H}), 2.01(\mathrm{dtd}, J=13.8,7.5,3.0 \mathrm{~Hz}$, 1H). ${ }^{13} \mathrm{C}$ NMR $\left(125 \mathrm{MHz}, \mathrm{CDCl}_{3}\right) \delta 206.87,140.64,132.66,129.55,129.20,127.58,127.35,86.44,57.75,46.18$, 33.70, 29.22. IR (neat, $\mathrm{cm}^{-1}$ ): 2932, 2827, 1720, 1643, 1490, 1453, 1277, 1196, 1117, 1059, 759. ESI ${ }^{+}$calculated for $\left[\mathrm{C}_{12} \mathrm{H}_{14} \mathrm{NaO}_{2}\right]^{+}: 213.09$, found: 213.09 . 
8-(Phenylsulfonyl)-8,9-dihydro-5H-benzo[7]annulen-6(7H)-one (3d)

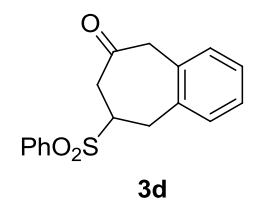

Compound 3d was prepared in $62 \%$ yield according to the above representative procedure (eluents: ethyl acetate/hexanes = 1/3). ${ }^{1} \mathrm{H}$ NMR $\left(500 \mathrm{MHz}, \mathrm{CDCl}_{3}\right) \delta 7.91(\mathrm{~d}, J=7.5 \mathrm{~Hz}, 2 \mathrm{H}), 7.70(\mathrm{t}, J=7.4 \mathrm{~Hz}, 1 \mathrm{H}), 7.60(\mathrm{t}, J=$ $7.8 \mathrm{~Hz}, 2 \mathrm{H}), 7.29-7.20(\mathrm{~m}, 3 \mathrm{H}), 7.17-7.10(\mathrm{~m}, 1 \mathrm{H}), 3.98(\mathrm{~d}, J=15.2 \mathrm{~Hz}, 1 \mathrm{H}), 3.55(\mathrm{~d}, J=15.3 \mathrm{~Hz}, 1 \mathrm{H}), 3.51-3.39$ $(\mathrm{m}, 3 \mathrm{H}), 2.88(\mathrm{dd}, J=13.3,9.0 \mathrm{~Hz}, 1 \mathrm{H}), 2.63(\mathrm{dd}, J=13.3,4.9 \mathrm{~Hz}, 1 \mathrm{H}) .{ }^{13} \mathrm{C}$ NMR $\left(125 \mathrm{MHz}, \mathrm{CDCl}_{3}\right) \delta 202.82$, 136.58, 135.33, 134.26, 133.36, 130.16, 129.71, 129.47, 129.04, 128.35, 128.18, 61.39, 49.54, 42.56, 31.19. IR (neat, $\left.\mathrm{cm}^{-1}\right)$ : 3066, 3025, 2967, 1713, 1491, 1447, 1302, 1146, 1084, 753, 729. ESI calculated for $\left[\mathrm{C}_{17} \mathrm{H}_{16} \mathrm{NaO}_{3} \mathrm{~S}\right]^{+}: 323.07$, found: 323.08 .

\section{3-Methyl-8-(phenylsulfonyl)-8,9-dihydro-5H-benzo[7]annulen-6(7H)-one (3e)}

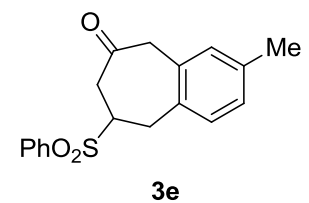

Compound 3e was prepared in $78 \%$ yield according to the above representative procedure (eluents: ethyl acetate/hexanes = 1/3). ${ }^{1} \mathrm{H}$ NMR $\left(500 \mathrm{MHz}, \mathrm{CDCl}_{3}\right) \delta 7.92-7.87(\mathrm{~m}, 2 \mathrm{H}), 7.70(\mathrm{t}, J=7.4 \mathrm{~Hz}, 1 \mathrm{H}), 7.60(\mathrm{t}, J=7.8 \mathrm{~Hz}$, 2H), $7.10(\mathrm{~d}, J=7.6 \mathrm{~Hz}, 1 \mathrm{H}), 7.04(\mathrm{dd}, J=7.7,1.7 \mathrm{~Hz}, 1 \mathrm{H}), 6.95(\mathrm{~s}, 1 \mathrm{H}), 3.94(\mathrm{~d}, J=15.0 \mathrm{~Hz}, 1 \mathrm{H}), 3.49$ (d, $J=15.1$ $\mathrm{Hz}, 1 \mathrm{H}), 3.47-3.35(\mathrm{~m}, 3 \mathrm{H}), 2.87(\mathrm{dd}, J=13.2,8.9 \mathrm{~Hz}, 1 \mathrm{H}), 2.63(\mathrm{dd}, J=13.2,4.7 \mathrm{~Hz}, 1 \mathrm{H}), 2.30(\mathrm{~s}, 3 \mathrm{H}) .{ }^{13} \mathrm{C}$ NMR $\left(125 \mathrm{MHz}, \mathrm{CDCl}_{3}\right) \delta 203.04,138.18,136.64,134.22,133.06,132.22,130.48,130.05,129.45,129.02,128.70,61.62$, 49.55, 42.68, 30.83, 20.94. IR (neat, $\mathrm{cm}^{-1}$ ): 3066, 2921, 2858, 1712, 1504, 1447, 1304, 1146, 1084, 834, 763, 739. $\mathrm{ESI}^{+}$calculated for $\left[\mathrm{C}_{18} \mathrm{H}_{18} \mathrm{NaO}_{3} \mathrm{~S}\right]^{+}: 337.09$, found: 337.07 .

\section{1-Methyl-8-(phenylsulfonyl)-8,9-dihydro-5H-benzo[7]annulen-6(7H)-one (3f)}
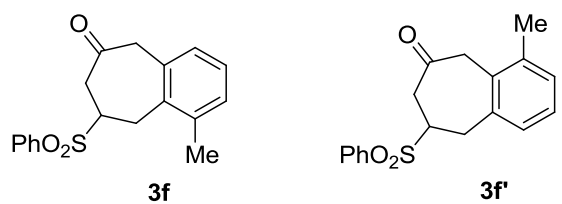

Compounds 3f/3f' were prepared in $70 \%$ total yield according to the above representative procedure (eluents: ethyl acetate/hexanes $=1 / 3)$. They exist as an inseparable mixture $\left(\mathbf{3 f} / \mathbf{3 f}{ }^{\prime}=3.9 / 1\right.$, determined by the crude $\left.{ }^{1} \mathrm{H} N \mathrm{NR}\right)$. After recrystallization from ethyl acetate/hexane, the ratio was improved to $>7 / 1$. The combined chemical shifts are reported, and the integration of the methyl peak from $3 \mathrm{f}$ is set to $3 .{ }^{1} \mathrm{H} \mathrm{NMR}\left(500 \mathrm{MHz}, \mathrm{CDCl}_{3}\right) \delta 7.97-7.88(\mathrm{~m}, 2.77 \mathrm{H}), 7.74-$ $7.66(\mathrm{~m}, 1.39 \mathrm{H}), 7.65-7.55(\mathrm{~m}, 2.76 \mathrm{H}), 7.14-7.08(\mathrm{~m}, 2.65 \mathrm{H}), 7.05-7.04(\mathrm{~m}, 0.35 \mathrm{H}), 6.98(\mathrm{t}, J=4.2 \mathrm{~Hz}, 1 \mathrm{H}), 4.00$ $(\mathrm{d}, J=15.0 \mathrm{~Hz}, 1 \mathrm{H}), 3.85(\mathrm{~d}, J=15.2 \mathrm{~Hz}, 0.27 \mathrm{H}), 3.76(\mathrm{~d}, J=15.3 \mathrm{~Hz}, 0.3 \mathrm{H}), 3.65-3.55(\mathrm{~m}, 1.05 \mathrm{H}), 3.50(\mathrm{~d}, J=15.1$ $\mathrm{Hz}, 1 \mathrm{H}), 3.45-3.28(\mathrm{~m}, 2.79 \mathrm{H}), 2.91-2.84(\mathrm{~m}, 1.35 \mathrm{H}), 2.63(\mathrm{ddd}, J=31.9,13.8,5.2 \mathrm{~Hz}, 1 \mathrm{H}), 2.60(\mathrm{dd}, J=13.4,5.5$ 
$\mathrm{Hz}, 0.27 \mathrm{H}), 2.33(\mathrm{~s}, 0.76 \mathrm{H}), 2.24(\mathrm{~s}, 3 \mathrm{H}) .{ }^{13} \mathrm{C} \mathrm{NMR}\left(125 \mathrm{MHz}, \mathrm{CDCl}_{3}\right) \delta 202.96,202.71,136.66,136.59,135.71$, $134.26,134.22$, 133.89, 133.70, 131.87, 130.24, 130.13, 129.48, 129.46, 129.45, 129.08, 129.03, 129.01, 127.97, 127.77, 127.65, 127.61, 77.25, 77.00, 76.75, 61.40, 60.88, 49.87, 44.60, 42.58, 42.36, 31.38, 26.32, 20.30, 19.72. IR (neat, $\mathrm{cm}^{-1}$ ): 3058, 2923, 1713, 1583, 1447, 1306, 1146, 1306, 1084, 781. ESI ${ }^{+}$calculated for $\left[\mathrm{C}_{18} \mathrm{H}_{18} \mathrm{NaO}_{3} \mathrm{~S}^{+}: 337.09\right.$, found: 337.08 .

4-Methyl-8-(phenylsulfonyl)-8,9-dihydro-5H-benzo[7]annulen-6(7H)-one (3g)

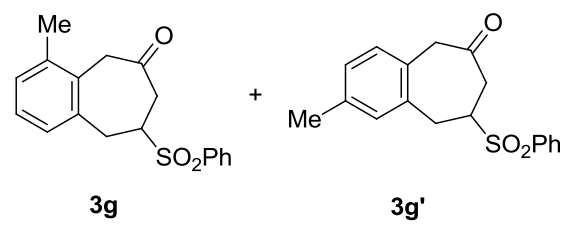

Compounds $\mathbf{3 g} / \mathbf{3 g}$ ' were prepared in $78 \%$ total yield according to the above representative procedure (eluents: ethyl acetate/hexanes $=1 / 3)$. They exist as an inseparable mixture $(\mathbf{3 g} / \mathbf{3 g} \mathbf{g}=2.5 / 3)$. The combined chemical shifts are reported, and the integration of the methyl peak from $3 \mathrm{~g}$ is set to $3 .{ }^{1} \mathrm{H} \mathrm{NMR}\left(500 \mathrm{MHz}, \mathrm{CDCl}_{3}\right) \delta 7.96-7.86(\mathrm{~m}$, $3.88 \mathrm{H}), 7.73-7.66(\mathrm{~m}, 1.95 \mathrm{H}), 7.64-7.56(\mathrm{~m}, 3.89 \mathrm{H}), 7.11(\mathrm{~d}, J=4.6 \mathrm{~Hz}, 1.86 \mathrm{H}), 7.08-6.99(\mathrm{~m}, 3.96 \mathrm{H}), 3.92(\mathrm{~d}, J=$ $15.2 \mathrm{~Hz}, 0.96 \mathrm{H}), 3.85(\mathrm{~d}, J=15.2 \mathrm{~Hz}, 0.89 \mathrm{H}), 3.76(\mathrm{~d}, J=15.2 \mathrm{~Hz}, 0.85 \mathrm{H}), 3.55-3.32(\mathrm{~m}, 6.54 \mathrm{H}), 2.89-2.84(\mathrm{~m}$, 1.89H), 2.65-2.56 (m, 1.91H), 2.33 (s, 2.5H), 2.31 (s, 3H). $\left.{ }^{13} \mathrm{C} \mathrm{NMR} \mathrm{(125} \mathrm{MHz,} \mathrm{CDCl}_{3}\right) \delta 203.11,202.97,137.98$, $136.65,136.59,136.53,135.71,135.13,134.24,134.22,131.87,130.91,130.25,130.18,129.60,129.45,129.04$, 129.01, 128.87, 127.97, 127.61, 61.54, 61.41, 49.11, 44.60, 42.68, 42.37, 31.38, 31.19, 20.92, 20.30. IR (neat, $\left.\mathrm{cm}^{-1}\right)$ : 2960, 2927, 2850, 1709, 1639, 1447, 1306, 1146, 1087. ESI calculated for $\left[\mathrm{C}_{18} \mathrm{H}_{18} \mathrm{NaO}_{3} \mathrm{~S}\right]^{+}: 337.09$, found: 337.09 .

\section{3-Chloro-8-(phenylsulfonyl)-8,9-dihydro-5H-benzo[7]annulen-6(7H)-one (3h)}
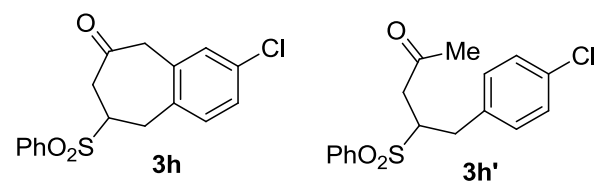

Compounds $\mathbf{3 h} / \mathbf{3 h}$ ' were prepared according to the above representative procedure (eluents: ethyl acetate/hexanes $=$ 1/3). 3h/3h' were separated by preparative TLC. 3h was isolated in $19 \%$ yield. ${ }^{1} \mathrm{H} \mathrm{NMR}\left(500 \mathrm{MHz}, \mathrm{CDCl}_{3}\right) \delta 7.89(\mathrm{~d}$, $J=7.8 \mathrm{~Hz}, 2 \mathrm{H}), 7.71(\mathrm{t}, J=7.4 \mathrm{~Hz}, 1 \mathrm{H}), 7.60(\mathrm{t}, J=7.7 \mathrm{~Hz}, 2 \mathrm{H}), 7.25-7.11(\mathrm{~m}, 3 \mathrm{H}), 3.91(\mathrm{~d}, J=15.7 \mathrm{~Hz}, 1 \mathrm{H}), 3.53$ $(\mathrm{d}, J=15.7 \mathrm{~Hz}, 1 \mathrm{H}), 3.48-3.33(\mathrm{~m}, 3 \mathrm{H}), 2.91-2.79(\mathrm{~m}, 1 \mathrm{H}), 2.60(\mathrm{dd}, J=13.3,4.9 \mathrm{~Hz}, 1 \mathrm{H}) .{ }^{13} \mathrm{C} \mathrm{NMR}(125 \mathrm{MHz}$, $\left.\mathrm{CDCl}_{3}\right) \delta 202.07,136.45,135.17,134.37,133.91,133.69,131.53,129.65,129.53,129.01,128.14,61.02,49.10,42.25$, 30.46. IR (neat, $\mathrm{cm}^{-1}$ ): 3066, 2971, 2923, 2847, 1713, 1642, 1487, 1448, 1305, 1146, 1084, 904, 830, 732. ESI calculated for $\left[\mathrm{C}_{17} \mathrm{H}_{15} \mathrm{ClNaO}_{3} \mathrm{~S}^{+}:\right.$357.03, found: 357.05 . 3h' was isolated in $36 \%$ yield. ${ }^{1} \mathrm{H} \mathrm{NMR}\left(500 \mathrm{MHz}, \mathrm{CDCl}_{3}\right)$

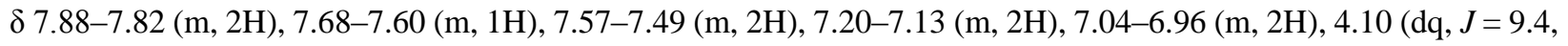
$5.7 \mathrm{~Hz}, 1 \mathrm{H}), 3.22-3.02(\mathrm{~m}, 2 \mathrm{H}), 2.78(\mathrm{dd}, J=14.3,9.5 \mathrm{~Hz}, 1 \mathrm{H}), 2.49(\mathrm{dd}, J=18.2,5.5 \mathrm{~Hz}, 1 \mathrm{H}), 2.02(\mathrm{~s}, 3 \mathrm{H}) .{ }^{13} \mathrm{C}$ $\operatorname{NMR}\left(125 \mathrm{MHz}, \mathrm{CDCl}_{3}\right) \delta 203.51,137.73,134.50,133.90,133.01,130.54,129.26,128.77,128.66,60.40,40.31$, 
33.87, 30.01. IR (neat, $\mathrm{cm}^{-1}$ ): 3069, 2926, 1719, 1643, 1493, 1447, 1411, 1305, 1087, 1016, 735. ESI ${ }^{+}$calculated for $\left[\mathrm{C}_{17} \mathrm{H}_{17} \mathrm{ClNaO}_{3} \mathrm{~S}\right]^{+}: 359.05$, found: 359.04 .

Diethyl 8-oxo-8,9-dihydro-5H-benzo[7]annulene-6,6(7H)-dicarboxylate (3i)

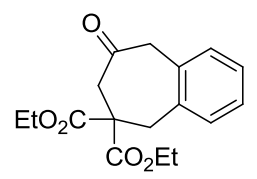

3i

Compound $3 \mathbf{i}$ was prepared in $78 \%$ yield according to the above representative procedure with $2.5 \%$ gold catalyst (eluents: ethyl acetate/hexanes = 1/10). ${ }^{1} \mathrm{H}$ NMR $\left(500 \mathrm{MHz}, \mathrm{CDCl}_{3}\right) \delta 7.25-7.19(\mathrm{~m}, 2 \mathrm{H}), 7.19-7.12(\mathrm{~m}, 2 \mathrm{H}), 4.18$ (q, $J=7.1 \mathrm{~Hz}, 4 \mathrm{H}), 3.74(\mathrm{~s}, 2 \mathrm{H}), 3.50(\mathrm{~s}, 2 \mathrm{H}), 2.89(\mathrm{~s}, 2 \mathrm{H}), 1.23(\mathrm{t}, J=7.1 \mathrm{~Hz}, 6 \mathrm{H}) \cdot{ }^{13} \mathrm{C} \mathrm{NMR}\left(125 \mathrm{MHz}, \mathrm{CDCl}_{3}\right) \delta 204.57$, 169.90, 135.05, 134.34, 130.37, 129.41, 127.99, 127.69, 62.07, 56.87, 49.40, 46.25, 37.32, 13.95. IR (neat, $\mathrm{cm}^{-1}$ ): 2983, 2931, 1733, 1602, 1448, 1368, 1193, 864. $\mathrm{ESI}^{+}$calculated for $\left[\mathrm{C}_{17} \mathrm{H}_{20} \mathrm{NaO}_{5}\right]^{+}: 327.12$, found: 327.11 .

\section{2-Tosyl-2,3-dihydro-1H-benzo[c]azepin-4(5H)-one (3j)}

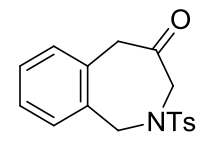

3j

Compound $\mathbf{3} \mathbf{j}$ was prepared in $57 \%$ yield according to the above representative procedure with the second step reaction temperature raised to $100^{\circ} \mathrm{C}$ (eluents: ethyl acetate/hexanes $=1 / 10$ ). $\mathbf{3 j}$ was further purified by recrystallized from ethyl acetate/hexanes. ${ }^{1} \mathrm{H}$ NMR (500 MHz, $\left.\mathrm{CDCl}_{3}\right) \delta 7.70(\mathrm{~d}, J=8.2 \mathrm{~Hz}, 2 \mathrm{H}), 7.34(\mathrm{~d}, J=8.0 \mathrm{~Hz}, 2 \mathrm{H}), 7.25-7.18$ (m, 2H), 7.12-7.05 (m, 2H), 4.67 (s, 2H), 3.97 (s, 2H), 3.96 (s, 2H), 2.44 (s, 3H). ${ }^{13} \mathrm{C}$ NMR (125 MHz, CDCl $) \delta 204.38$, 144.09, 135.30, 133.07, 131.17, 130.31, 130.01, 128.19, 128.03, 127.72, 127.08, 57.05, 52.70, 48.35, 21.55. IR (neat, $\mathrm{cm}^{-1}$ ): 3066, 2960, 2903, 1728, 1597, 1496, 1437, 1345, 1259, 1160, 1092, 928, 817, 757. ESI ${ }^{+}$calculated for $\left[\mathrm{C}_{17} \mathrm{H}_{17} \mathrm{NNaO}_{3} \mathrm{~S}+\mathrm{MeOH}\right]^{+}: 370.11$, found: 370.09 .

\section{3,5-Dihydrobenzo[c]oxepin-4(1H)-one (3k)}

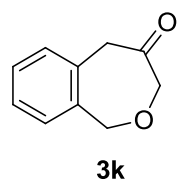

Compound 3k was prepared in 52\% yield according to the above representative procedure with the second step reaction temperature raised to $100^{\circ} \mathrm{C}$ (eluents: ethyl acetate/hexanes $\left.=1 / 10\right) .{ }^{1} \mathrm{H}$ NMR $\left(500 \mathrm{MHz}, \mathrm{CDCl}_{3}\right) \delta 7.25-7.18$ (m, 2H), $7.11(\mathrm{~d}, J=7.4 \mathrm{~Hz}, 1 \mathrm{H}), 7.03(\mathrm{~d}, J=7.4 \mathrm{~Hz}, 1 \mathrm{H}), 5.11(\mathrm{~s}, 2 \mathrm{H}), 4.19(\mathrm{~s}, 2 \mathrm{H}), 4.08(\mathrm{~s}, 2 \mathrm{H}) .{ }^{13} \mathrm{C}$ NMR $(125$ $\left.\mathrm{MHz}, \mathrm{CDCl}_{3}\right) \delta$ 209.07, 135.77, 130.94, 129.25, 127.70, 127.37, 125.92, 76.09, 74.90, 48.50. IR (neat, $\mathrm{cm}^{-1}$ ): 2925, $2852,1725,1496,1447,1263,1116,955,752$. ESI ${ }^{+}$calculated for $\left[\mathrm{C}_{10} \mathrm{H}_{10} \mathrm{NaO}_{2}+\mathrm{MeOH}\right]^{+}: 217.08$, found: 217.09 . 


\section{8-((Benzyloxy)methyl)-3,5-dihydrobenzo[c]oxepin-4(1H)-one (3n)}

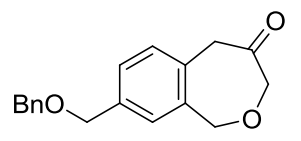

$3 n$

Compound 3n was prepared in $48 \%$ yield according to the above representative procedure with the second step reaction temperature raised to $100^{\circ} \mathrm{C}$ (eluents: ethyl acetate/hexanes $\left.=1 / 10\right) .{ }^{1} \mathrm{H} \mathrm{NMR}\left(500 \mathrm{MHz}, \mathrm{CDCl}_{3}\right) \delta 7.38-7.34$ (m, 4H), 7.32- $7.28(\mathrm{~m}, 1 \mathrm{H}), 7.25(\mathrm{~d}, \mathrm{~J}=7.9 \mathrm{~Hz}, 1 \mathrm{H}), 7.12(\mathrm{~s}, 1 \mathrm{H}), 7.02(\mathrm{~d}, \mathrm{~J}=7.9 \mathrm{~Hz}, 1 \mathrm{H}), 5.11(\mathrm{~s}, 2 \mathrm{H}), 4.55(\mathrm{~s}, 2 \mathrm{H})$, 4.50 (s, 2H), 4.18 (s, 2H), 4.08 (s, 2H). ${ }^{13} \mathrm{C}$ NMR (126 MHz, CDCl3) $\delta 208.96,138.05,137.95,135.13,130.26,129.34$, 128.43, 127.77, 127.70, 126.80, 126.11, 76.09, 74.78, 72.32, 71.45, 48.47. IR (neat, $\mathrm{cm}^{-1}$ ): 3064, 2926, 1721, 1614, 1452, 1385, 1272, 1120, 1038, 945, 846. ESI $^{+}$calculated for $\left[\mathrm{C}_{18} \mathrm{H}_{18} \mathrm{NaO}_{3}+\mathrm{MeOH}\right]^{+}: 337.14$ found: 337.10 .

\section{9-Bromo-8-methoxy-3,5-dihydrobenzo[c]oxepin-4(1H)-one (3o)}

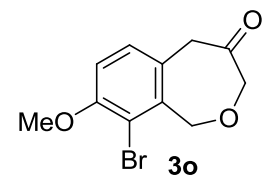

Compound 30 was prepared in 57\% yield according to the above representative procedure with the second step reaction temperature raised to $100^{\circ} \mathrm{C}$ (eluents: ethyl acetate/hexanes $\left.=1 / 10\right) .{ }^{1} \mathrm{H} \mathrm{NMR}\left(500 \mathrm{MHz}, \mathrm{CDCl}_{3}\right) \delta 7.04(\mathrm{~d}, \mathrm{~J}$ $=8.5 \mathrm{~Hz}, 1 \mathrm{H}), 6.78(\mathrm{~d}, \mathrm{~J}=8.5 \mathrm{~Hz}, 1 \mathrm{H}), 5.06(\mathrm{~s}, 2 \mathrm{H}), 4.17(\mathrm{~s}, 2 \mathrm{H}), 4.05(\mathrm{~s}, 2 \mathrm{H}), 3.88(\mathrm{~s}, 3 \mathrm{H}) .{ }^{13} \mathrm{C} \mathrm{NMR}(126 \mathrm{MHz}$, CDCl3) $\delta 208.87,155.15,135.85,130.79,123.04,111.24,110.62,76.40,56.37,47.71,29.69$. IR (neat, $\mathrm{cm}^{-1}$ ): 2919 , $2849,1726,1596,1480,1414,1284,1108,1062,965,807 . \mathrm{ESI}^{+}$calculated for $\left[\mathrm{C}_{12} \mathrm{H}_{15} \mathrm{BrNaO}_{4}+\mathrm{MeOH}\right]^{+}: 325.01$, 327.01 found: $325.00,327.00$.

\section{(2) The representative procedure for the synthesis of $31,3 \mathrm{~m}$ and $3 \mathrm{p}$}

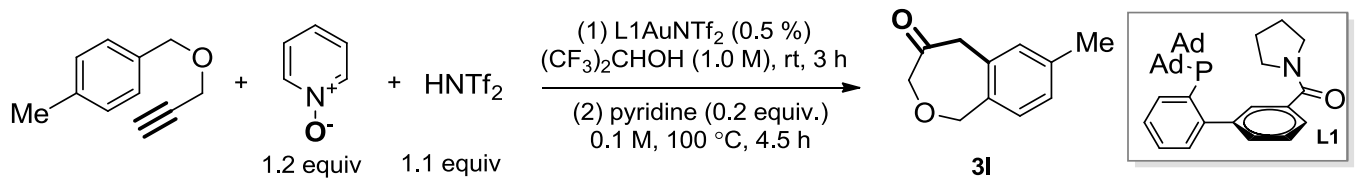

$\mathrm{L}_{1} \mathrm{AuNTf}_{2}$ (1.5 mg, 0.005 equiv.) was added into a mixture of the premixed salt (126.9 mg, 1.2 equiv. in pyridine $N$-oxide), alkyne $(0.3 \mathrm{mmol})$ and HFIP $(0.3 \mathrm{~mL})$ in a vial at room temperature. The reaction mixture was stirred at room temperature and the progress of the reaction was monitored by TLC (hexanes/ethyl acetate $=10 / 1$ ). Upon completion, the mixture was diluted with HFIP $(2.7 \mathrm{ml})$. Pyridine ( 0.2 equiv.) was added to the above reaction mixture. The vial was then sealed and heated to $100{ }^{\circ} \mathrm{C}$. The reaction finished in $4.5 \mathrm{~h}$, as indicated by TLC $(\mathrm{DCM} / \mathrm{MeOH}=$ 10/1). The reaction mixture was concentrated, and the resulting residue was purified by chromatography on silica gel (eluent: hexanes/ethyl acetate) to afford the desired product. 
7-Methyl-3,5-dihydrobenzo[c]oxepin-4(1H)-one (3I)

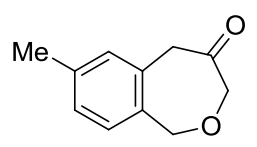

31

Compound 31 was prepared in 65\% yield according to the above representative procedure (eluents: ethyl acetate/hexanes = 1/10). ${ }^{1} \mathrm{H}$ NMR $\left(500 \mathrm{MHz}, \mathrm{CDCl}_{3}\right) \delta 7.05(\mathrm{dd}, J=8.0,1.6 \mathrm{~Hz}, 1 \mathrm{H}), 6.94-6.90(\mathrm{~m}, 2 \mathrm{H}), 5.08(\mathrm{~s}$, 2H), 4.17 (s, 2H), 4.03 (s, 2H), 2.30 (s, 3H). $\left.{ }^{13} \mathrm{C} \mathrm{NMR} \mathrm{(125} \mathrm{MHz,} \mathrm{CDCl}_{3}\right) \delta 209.17,137.46,132.63,131.45,129.08$, 128.14, 125.94, 75.95, 74.74, 48.44, 20.83. IR (neat, $\mathrm{cm}^{-1}$ ): 2960, 2934, 2876, 1734, 1613, 1453, 1260, 1166, 963, 751. $\mathrm{ESI}^{+}$calculated for $\left[\mathrm{C}_{11} \mathrm{H}_{12} \mathrm{NaO}_{2}+\mathrm{MeOH}\right]^{+}: 231.10$, found: 231.08 .

\section{3-Methyl-3,5-dihydrobenzo[c]oxepin-4(1H)-one (3m)}

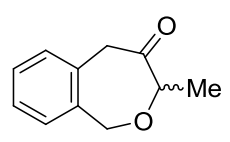

$3 \mathrm{~m}$

Compound $3 \mathrm{~m}$ was prepared in $64 \%$ yield according to the above representative procedure (eluents: ethyl acetate/hexanes = 1/10). ${ }^{1} \mathrm{H}$ NMR $\left(500 \mathrm{MHz}, \mathrm{CDCl}_{3}\right) \delta 7.24-7.18(\mathrm{~m}, 2 \mathrm{H}), 7.12(\mathrm{~d}, J=7.0,1 \mathrm{H}), 7.02(\mathrm{~d}, J=7.4 \mathrm{~Hz}$, $1 \mathrm{H}), 5.31(\mathrm{~d}, J=16.0 \mathrm{~Hz}, 1 \mathrm{H}), 4.86(\mathrm{~d}, J=16.0 \mathrm{~Hz}, 1 \mathrm{H}), 4.71(\mathrm{~d}, J=12.3 \mathrm{~Hz}, 1 \mathrm{H}), 4.01(\mathrm{q}, J=7.0 \mathrm{~Hz}, 1 \mathrm{H}), 3.37(\mathrm{~d}$, $J=12.2 \mathrm{~Hz}, 1 \mathrm{H}), 1.42(\mathrm{~d}, J=7.0 \mathrm{~Hz}, 3 \mathrm{H}) .{ }^{13} \mathrm{C} \mathrm{NMR}\left(125 \mathrm{MHz}, \mathrm{CDCl}_{3}\right) \delta 210.73,136.02,130.79,129.63,127.57$, 127.21, 125.74, 82.19, 73.65, 46.87, 17.92. IR (neat, $\mathrm{cm}^{-1}$ ): 2989, 2923, 1644, 1451, 1379, 1287, 1255, 1111, 741. $\mathrm{ESI}^{+}$calculated for $\left[\mathrm{C}_{11} \mathrm{H}_{12} \mathrm{NaO}_{2}\right]^{+}:$199.07, found: 199.09 .

\section{(R)-3-Methyl-3,5-dihydrobenzo[c]oxepin-4(1H)-one (3m')}

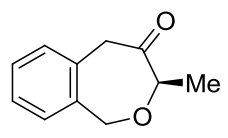

$3 m^{\prime}$

Compound 3m was prepared in $64 \%$ yield according to the above representative procedure (eluents: ethyl acetate/hexanes $=1 / 10)$. The spectra data were identical with the racemic one $(\mathbf{3 m})$ above. The enantiomeric excess is determined by chiral HPLC [Chiral Cel OJ-H Column, isopropanol : hexane = $1: 19(1.0 \mathrm{~mL} / \mathrm{min})$ ] to be $99 \%$.

\section{4-Oxo-1,3,4,5-tetrahydrobenzo[c]oxepin-8-yl pivalate (3p)}

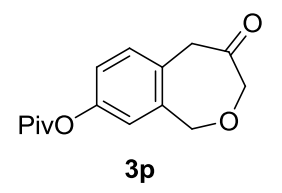


Compound 3p was prepared in 51\% yield according to the above representative procedure (eluents: ethyl acetate/hexanes = 1/10). ${ }^{1} \mathrm{H}$ NMR $\left(500 \mathrm{MHz}, \mathrm{CDCl}_{3}\right) \delta 7.04(\mathrm{~d}, \mathrm{~J}=8.4 \mathrm{~Hz}, 1 \mathrm{H}), 6.95(\mathrm{~d}, \mathrm{~J}=8.5 \mathrm{~Hz}, 1 \mathrm{H}), 6.82(\mathrm{~s}, 1 \mathrm{H})$, 5.09 (s, 2H), 4.19 (s, 2H), 4.05 (s, 2H), 1.35 (s, 9H). ${ }^{13} \mathrm{C}$ NMR (126 MHz, CDCl3) $\delta$ 208.40, 176.99, 150.27, 133.07, 130.67, 126.97, 123.64, 120.65, 76.11, 74.54, 48.26, 27.09. IR (neat, $\mathrm{cm}^{-1}$ ): 3064, 2976, 2876, 1752, 1609, 1481, 1275, $1236,1117,1030,911,737$. ESI ${ }^{+}$calculated for $\left[\mathrm{C}_{15} \mathrm{H}_{18} \mathrm{NaO}_{4}\right]^{+}: 285.11$, found: 285.11 .

(3) Representative procedure for the synthesis of benzene-fused 8-membered ketones

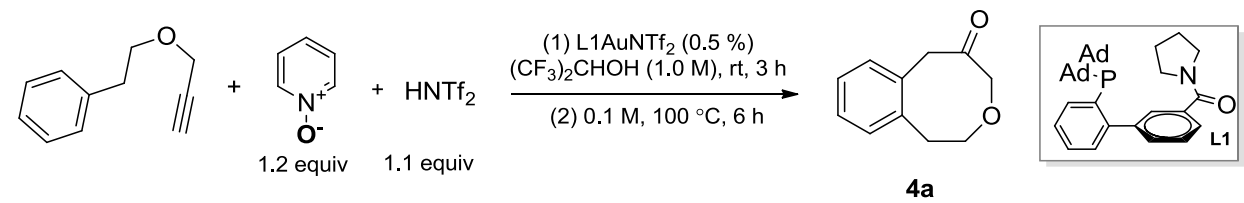

$\mathrm{L}_{1} \mathrm{AuNTf}_{2}$ (1.5 mg, 0.005 equiv.) was added into a mixture of premixed salt (126.9 mg, 1.2 equiv.), alkyne (0.3 $\mathrm{mmol})$, HFIP $(0.3 \mathrm{~mL})$ in a vial at room temperature. The reaction mixture was then stirred at room temperature and the progress of the reaction was monitored by TLC (PE/EA $=10 / 1$, visualization reagent: $\mathrm{KMnO}_{4} / \mathrm{H}_{2} \mathrm{SO}_{4}$ ). Upon completion, the mixture was diluted with HFIP $(2.7 \mathrm{ml})$. Then the vial was sealed and heated to $100^{\circ} \mathrm{C}$. The reaction was finished in $6 \mathrm{~h}$ which was monitored by TLC (DCM/MeOH $=10 / 1$, visualization reagent: $\left.\mathrm{KMnO}_{4} / \mathrm{H}_{2} \mathrm{SO}_{4}\right)$. After the reaction was finished, the mixture was concentrated and the residue was purified by chromatography on silica gel (eluent: hexanes/ethyl acetate) to afford the desired product $\mathbf{4 a}$.

\section{4,6-Dihydro-1H-benzo[d]oxocin-5(2H)-one (4a)}

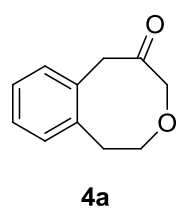

Compound 4a was prepared in $66 \%$ yield according to the above representative procedure (eluents: ethyl acetate/hexanes = 1/10). ${ }^{1} \mathrm{H}$ NMR $\left(500 \mathrm{MHz}, \mathrm{CDCl}_{3}\right) \delta 7.25-7.21(\mathrm{~m}, 2 \mathrm{H}), 7.16(\mathrm{dd}, J=6.4,2.4 \mathrm{~Hz}, 0 \mathrm{H}), 4.07-3.93$ (m, 2H), $3.92(\mathrm{~s}, 1 \mathrm{H}), 3.08(\mathrm{t}, J=4.8 \mathrm{~Hz}, 1 \mathrm{H}) .{ }^{13} \mathrm{C} \mathrm{NMR}\left(125 \mathrm{MHz}, \mathrm{CDCl}_{3}\right) \delta 210.93,139.09,132.23,130.50,127.91$, 127.61, 77.29, 76.57, 45.97, 38.67. IR (neat, $\mathrm{cm}^{-1}$ ): 3022, 2941, 2869, 1715, 1492, 1453, 1272, 1124, 759. ESI calculated for $\left[\mathrm{C}_{11} \mathrm{H}_{12} \mathrm{NaO}_{2}+\mathrm{MeOH}\right]^{+}: 231.10$, found: 231.10 .

\section{8-Methyl-4,6-dihydro-1H-benzo[d]oxocin-5(2H)-one (4b)}

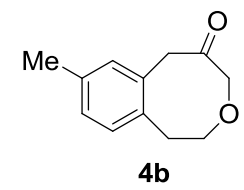

Compound $4 \mathbf{b}$ was prepared in $74 \%$ yield according to the above representative procedure (eluents: ethyl acetate/hexanes = 1/10). ${ }^{1} \mathrm{H}$ NMR $\left(500 \mathrm{MHz}, \mathrm{CDCl}_{3}\right) \delta 7.08-7.01(\mathrm{~m}, 3 \mathrm{H}), 3.95(\mathrm{~s}, 4 \mathrm{H}), 3.92(\mathrm{~s}, 2 \mathrm{H}), 3.03(\mathrm{t}, J=4.8$ $\mathrm{Hz}, 2 \mathrm{H}), 2.30$ (s, 3H). ${ }^{13} \mathrm{C} \mathrm{NMR}\left(125 \mathrm{MHz}, \mathrm{CDCl}_{3}\right) \delta 211.19,137.32,136.07,131.99,131.19,130.44,128.63,77.39$, 
76.85, 45.96, 38.28, 20.82. IR (neat, $\mathrm{cm}^{-1}$ ): 2938, 2867, 1715, 1503, 1455, 1271, 1127, 1056, 964, 820. ESI ${ }^{+}$calculated for $\left[\mathrm{C}_{12} \mathrm{H}_{14} \mathrm{NaO}_{2}+\mathrm{MeOH}\right]^{+}: 245.12$, found: 245.12 .

\section{8,10-Dimethyl-4,6-dihydro-1H-benzo[d]oxocin-5(2H)-one (4c)}

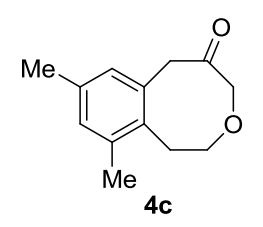

Compound 4c was prepared in $87 \%$ yield according to the above representative procedure (eluents: ethyl acetate/hexanes = 1/10). ${ }^{1} \mathrm{H} \mathrm{NMR}\left(500 \mathrm{MHz} \mathrm{CDCl}_{3}\right) \delta 6.92(\mathrm{~s}, 1 \mathrm{H}), 6.84(\mathrm{~s}, 1 \mathrm{H}), 3.99(\mathrm{brs}, 4 \mathrm{H}), 3.90(\mathrm{~s}, 2 \mathrm{H}), 3.04(\mathrm{t}$, $J=4.9 \mathrm{~Hz}, 2 \mathrm{H}), 2.36$ (s, 3H), 2.27 (s, 3H). ${ }^{13} \mathrm{C} \mathrm{NMR}\left(125 \mathrm{MHz}, \mathrm{CDCl}_{3}\right) \delta$ 210.68, 139.46, 138.29, 137.04, 130.24, 129.10, 127.65, 77.31, 76.89, 41.32, 39.11, 20.78, 20.04. IR (neat, $\mathrm{cm}^{-1}$ ): 2940, 2862, 1712, 1453, 1276, 1137, 1120, 913, 748. $\mathrm{ESI}^{+}$calculated for $\left[\mathrm{C}_{13} \mathrm{H}_{16} \mathrm{NaO}_{2}+\mathrm{MeOH}\right]^{+}: 259.13$, found: 259.13 .

9-Methoxy-4,6-dihydro-1H-benzo[d]oxocin-5(2H)-one (4d)
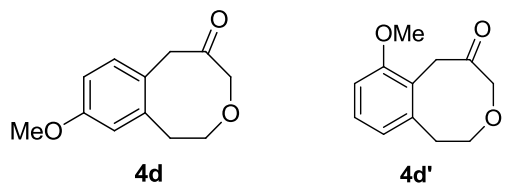

Compounds $4 \mathbf{d}$ and $\mathbf{4 d}$ ' were prepared in $82 \%$ total yield according to the above representative procedure (eluents: ethyl acetate/hexanes $=1 / 10)$. They exist as an inseparable mixture $\left(\mathbf{4 d} / \mathbf{4} \mathbf{d} '=3 / 1.2\right.$, determined by ${ }^{1} \mathrm{H}$ NMR). The combined chemical shifts are reported, and the integration of the methoxyl group from $4 \mathbf{d}$ is set to $3 .{ }^{1} \mathrm{H}$ NMR $(500$ $\left.\mathrm{MHz}_{\mathrm{CDCl}}\right) \delta 7.18(\mathrm{t}, J=7.9 \mathrm{~Hz}, 0.43 \mathrm{H}), 7.15(\mathrm{~d}, J=8.4 \mathrm{~Hz}, 0.95 \mathrm{H}), 6.81-6.73(\mathrm{~m}, 0.79 \mathrm{H}), 6.71(\mathrm{~d}, J=2.7 \mathrm{~Hz}$ $0.96 \mathrm{H}), 4.04(\mathrm{~s}, 0.87 \mathrm{H}), 3.98-3.86(\mathrm{~m}, 7.36 \mathrm{H}), 3.83(\mathrm{~s}, 1.2 \mathrm{H}), 3.78(\mathrm{~s}, 3 \mathrm{H}), 3.03(\mathrm{~m}, 2.83 \mathrm{H})$. For ${ }^{13} \mathrm{C}$ NMR the peaks of $4 \mathbf{d}$ and $\mathbf{4 d}$ ' can be identified and reported as follows. $4 \mathbf{d}:{ }^{13} \mathrm{C}$ NMR $\left(125 \mathrm{MHz}, \mathrm{CDCl}_{3}\right) \delta 211.07,159.19,140.44$, 131.60, 122.45, 116.14, 112.58, 77.29, 76.58, 55.21, 44.95, 38.96. 4d': ${ }^{13} \mathrm{C}$ NMR (125 MHz, $\left.\mathrm{CDCl}_{3}\right) \delta 210.39,158.10$, 140.49, 128.30, 124.27, 121.21, 109.27, 77.47, 76.22, 55.74, 38.49, 38.40. IR (neat, $\mathrm{cm}^{-1}$ ): 2941, 2872, 1713, 1596, 1498, 1470, 1263, 1125, 1038, 827. $\mathrm{ESI}^{+}$calculated for $\left[\mathrm{C}_{12} \mathrm{H}_{14} \mathrm{NaO}_{3}+\mathrm{MeOH}\right]^{+}:$261.11, found: 261.10 .

\section{0-Methyl-4,6-dihydro-1H-benzo[d]oxocin-5(2H)-one (4e)}

Compounds $\mathbf{4 e}$ and $\mathbf{4 e}$ ' were prepared in $80 \%$ total yield according to the above representative procedure (eluents: ethyl acetate/hexanes $=1 / 10)$. $4 \mathbf{e}$ and $\mathbf{4 e}$ ' $\left(3 / 1\right.$, determined by ${ }^{1} \mathrm{H}$ NMR) were separated by preparative TLC.

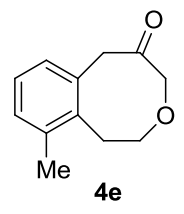

${ }^{1} \mathrm{H}$ NMR $\left(500 \mathrm{MHz} \mathrm{CDCl}_{3}\right) \delta 7.15-7.10(\mathrm{~m}, 1 \mathrm{H}), 7.09(\mathrm{~d}, J=4.9 \mathrm{~Hz}, 2 \mathrm{H}), 4.08-3.93(\mathrm{~m}, 4 \mathrm{H}), 3.89(\mathrm{~s}, 2 \mathrm{H}), 3.16-$ $3.08(\mathrm{~m}, 2 \mathrm{H}), 2.33$ (s, 3H). ${ }^{13} \mathrm{C}$ NMR $\left(125 \mathrm{MHz}, \mathrm{CDCl}_{3}\right) \delta 210.68,137.15,136.46,132.76,129.83,128.35,127.07$, 
76.97, 75.38, 46.62, 32.87, 20.86. IR (neat, $\mathrm{cm}^{-1}$ ): 2944, 2872, 1712, 1464, 1269, 1211, 1126, 762. ESI ${ }^{+}$calculated for $\left[\mathrm{C}_{12} \mathrm{H}_{14} \mathrm{NaO}_{2}+\mathrm{MeOH}\right]^{+}:$245.12, found: 245.10 .

\section{7-methyl-4,6-dihydro-1H-benzo[d]oxocin-5(2H)-one (4e')}

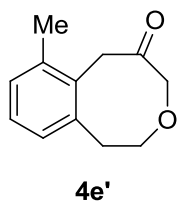

${ }^{1} \mathrm{H}$ NMR (600 MHz, $\left.\mathrm{CDCl}_{3}\right) \delta$ 7.12-7.07 (m, 2H), 7.00 (d, $\left.J=7.0 \mathrm{~Hz}, 1 \mathrm{H}\right), 4.04(\mathrm{~s}, 2 \mathrm{H}), 3.98(\mathrm{~s}, 2 \mathrm{H}), 3.89$ (s, 2H), $3.07(\mathrm{t}, J=4.9 \mathrm{~Hz}, 2 \mathrm{H}), 2.39(\mathrm{~s}, 3 \mathrm{H}) .{ }^{13} \mathrm{C} \mathrm{NMR}\left(125 \mathrm{MHz}, \mathrm{CDCl}_{3}\right) \delta 210.60,139.61,138.52,130.78,129.48,128.43$, 127.51, 77.37, 76.85, 41.75, 39.18, 20.20. IR (neat, $\mathrm{cm}^{-1}$ ): 2940, 2872, 1714, 1451, 1278, 1227, 1122, 777. ESI calculated for $\left[\mathrm{C}_{12} \mathrm{H}_{14} \mathrm{NaO}_{2}+\mathrm{MeOH}\right]^{+}: 245.12$, found: 245.12 .

\section{5,6-Dihydro-1H-naphtho[2,1-d]oxocin-2(3H)-one (4f)}
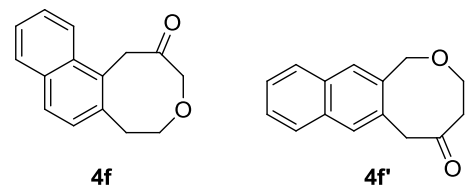

Compounds $\mathbf{4 f}$ and $\mathbf{4 f}$ ' were prepared in $82 \%$ total yield according to the above representative procedure (eluents: ethyl acetate/hexanes $=1 / 10)$. They exist as an inseparable mixture $\left(\mathbf{4 f} / \mathbf{4} \mathbf{f}^{\prime}=2.7 / 1\right.$, determined by ${ }^{1} \mathrm{H}$ NMR). The combined chemical shifts are reported. ${ }^{1} \mathrm{H}$ NMR $\left(500 \mathrm{MHz}, \mathrm{CDCl}_{3}\right) \delta 8.29(\mathrm{~d}, J=8.6 \mathrm{~Hz}, 1 \mathrm{H}), 7.82(\mathrm{dd}, J=8.1,1.3$ $\mathrm{Hz}, 1 \mathrm{H}), 7.80-7.73(\mathrm{~m}, 2 \mathrm{H}), 7.66(\mathrm{~s}, 0.38 \mathrm{H}), 7.58(\mathrm{ddd}, J=8.5,6.8,1.5 \mathrm{~Hz}, 1 \mathrm{H}), 7.51-7.42(\mathrm{~m}, 1.7 \mathrm{H}), 7.30(\mathrm{~d}, J=$ $8.4 \mathrm{~Hz}, 1 \mathrm{H}), 4.50(\mathrm{~s}, 2 \mathrm{H}), 4.16(\mathrm{~s}, 0.74 \mathrm{H}), 4.06(\mathrm{brs}, 2.67 \mathrm{H}), 3.93(\mathrm{~s}, 0.7 \mathrm{H}), 3.91(\mathrm{~s}, 2 \mathrm{H}), 3.27(\mathrm{~m}, 2.77 \mathrm{H})$. For ${ }^{13} \mathrm{C}$ NMR the peaks of $\mathbf{4 f}$ and $\mathbf{4 f}$ ' can be identified and reported as follows. $\mathbf{4 f}:{ }^{13} \mathrm{C}$ NMR $\left(125 \mathrm{MHz}, \mathrm{CDCl}_{3}\right) \delta 210.12$, 137.04, 132.98, 132.46, 129.51, 128.68, 128.40, 128.38, 126.96, 125.57, 124.20, 77.44, 76.41, 40.72, 39.56. 4f': ${ }^{13} \mathrm{C}$ NMR (125 MHz, $\left.\mathrm{CDCl}_{3}\right) \delta 210.60,137.27,132.97,132.78,130.46,129.04,127.36,127.32,127.06,126.19,126.01$, 77.41, 46.00, 38.92. IR (neat, $\mathrm{cm}^{-1}$ ): 3055, 2940, 2888, 1714, 1642, 1281, 1121, 819, 749. ESI ${ }^{+}$calculated for $\left[\mathrm{C}_{15} \mathrm{H}_{14} \mathrm{NaO}_{2}+\mathrm{MeOH}\right]^{+}:$281.12, found: 281.11 .

\section{4,6-Dihydro-1H-naphtho[1,2-d]oxocin-5(2H)-one (4g)}

Compounds $\mathbf{4 g}$ and $\mathbf{4 g}$ ' were isolated in $\mathbf{7 8 \%}$ yield according to the above representative procedure (eluents: ethyl acetate/hexanes $=1 / 10) . \mathbf{4 g} / \mathbf{4 g}$ ' $\left(2 / 1\right.$, determined by ${ }^{1} \mathrm{H}$ NMR $)$ were separated by preparative TLC.

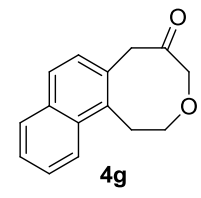

${ }^{1} \mathrm{H}$ NMR (500 MHz, $\left.\mathrm{CDCl}_{3}\right) \delta 8.03(\mathrm{~d}, J=8.5 \mathrm{~Hz}, 1 \mathrm{H}), 7.86(\mathrm{~d}, J=8.0 \mathrm{~Hz}, 1 \mathrm{H}), 7.74(\mathrm{~d}, J=8.5 \mathrm{~Hz}, 1 \mathrm{H}), 7.59-7.53$ (m, 1H), 7.53-7.47 (m, 1H), $7.36(\mathrm{~d}, J=8.4 \mathrm{~Hz}, 1 \mathrm{H}), 4.21(\mathrm{~s}, 2 \mathrm{H}), 4.10(\mathrm{t}, J=5.1 \mathrm{~Hz}, 2 \mathrm{H}), 3.90(\mathrm{~s}, 2 \mathrm{H}), 3.58(\mathrm{t}, J=$ 
$4.8 \mathrm{~Hz}, 2 \mathrm{H}) .{ }^{13} \mathrm{C}$ NMR $\left(126 \mathrm{MHz}, \mathrm{CDCl}_{3}\right) \delta 210.53,134.13,133.38,131.91,130.14,129.02,128.01,127.93,126.61$, 125.58, 123.23, 76.89, 75.46, 47.28, 31.55. IR (neat, $\mathrm{cm}^{-1}$ ): 2920, 2850, 1709, 1640, 1447, 1306, 1146, 1087, 762, 728. $\mathrm{ESI}^{+}$calculated for $\left[\mathrm{C}_{15} \mathrm{H}_{14} \mathrm{NaO}_{2}+\mathrm{MeOH}\right]^{+}: 281.12$, found: 281.12 .

\section{5,6-Dihydro-1H-naphtho[1,8-de]oxonin-2(3H)-one (4g')}

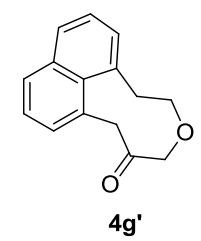

${ }^{1} \mathrm{H}$ NMR $\left(500 \mathrm{MHz}, \mathrm{CDCl}_{3}\right) \delta 7.77(\mathrm{td}, J=8.0,1.5 \mathrm{~Hz}, 2 \mathrm{H}), 7.43-7.28(\mathrm{~m}, 4 \mathrm{H}), 4.85(\mathrm{~d}, J=17.6 \mathrm{~Hz}, 1 \mathrm{H}), 4.35(\mathrm{~d}, J$ $=16.1 \mathrm{~Hz}, 1 \mathrm{H}), 4.06(\mathrm{~d}, J=14.6 \mathrm{~Hz}, 1 \mathrm{H}), 3.97(\mathrm{~d}, J=15.4 \mathrm{~Hz}, 2 \mathrm{H}), 3.89-3.58(\mathrm{~m}, 2 \mathrm{H}), 2.90(\mathrm{~d}, J=15.5 \mathrm{~Hz}, 1 \mathrm{H})$. ${ }^{13} \mathrm{C}$ NMR $\left(125 \mathrm{MHz}, \mathrm{CDCl}_{3}\right) \delta 211.09,136.86,135.23,134.26,130.99,130.78,129.85,129.14,128.96,125.56$, 125.03, 78.86, 74.94, 50.48, 38.31. IR (neat, $\left.\mathrm{cm}^{-1}\right): 3044,2923,2865,1705,1456,1251,1193,1114,830,773$. ESI $^{+}$ calculated for $\left[\mathrm{C}_{15} \mathrm{H}_{14} \mathrm{NaO}_{2}+\mathrm{MeOH}\right]^{+}: 281.12$, found: 281.11 .

\section{3-Tosyl-1,2,3,4-tetrahydrobenzo[d]azocin-5(6H)-one (4h)}

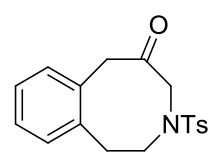

$4 h$

Compound $4 \mathrm{~h}$ was prepared in $78 \%$ yield according to the above representative procedure (eluents: ethyl acetate/hexanes = 1/10). ${ }^{1} \mathrm{H}$ NMR $\left(500 \mathrm{MHz}, \mathrm{CDCl}_{3}\right) \delta 7.69(\mathrm{~d}, J=8.1 \mathrm{~Hz}, 2 \mathrm{H}), 7.31(\mathrm{~d}, J=8.0 \mathrm{~Hz}, 2 \mathrm{H}), 7.25-7.18$ (m, 3H), 7.16-7.12 (m, 1H), 4.05 (s, 2H), 3.57 (brs, 2H), 3.53 (s, 2H), 3.0 (t, $J=4.0 \mathrm{~Hz}, 2 \mathrm{H}), 2.42(\mathrm{~s}, 3 \mathrm{H}) .{ }^{13} \mathrm{C}$ NMR $\left(125 \mathrm{MHz}, \mathrm{CDCl}_{3}\right) \delta 206.95,143.83,138.56,136.53,132.49,130.85,130.68,129.89,128.12,127.77,126.75,77.25$, 77.00, 76.75, 56.96, 53.34, 46.02, 38.30, 21.48. IR (neat, $\mathrm{cm}^{-1}$ ): 3062, 2919, 2850, 1718, 1642, 1493, 1448, 1340, $1157,1091,1018,924,887,816,740 . \mathrm{ESI}^{+}$calculated for $\left[\mathrm{C}_{18} \mathrm{H}_{19} \mathrm{NNaO}_{3} \mathrm{~S}\right]^{+}: 352.10$, found: 352.11 .

\section{3,4-Dihydro-2H-benzo[b]oxocin-5(6H)-one (4i)}

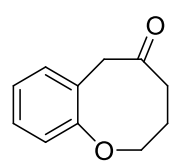

$4 \mathbf{i}$

Compound 4i was prepared in 57\% yield according to the above representative procedure (eluents: ethyl acetate/hexanes $=1 / 10) .{ }^{1} \mathrm{H}$ NMR $\left(500 \mathrm{MHz}, \mathrm{CDCl}_{3}\right) \delta 7.30-7.25(\mathrm{~m}, 1 \mathrm{H}), 7.16-7.13(\mathrm{~m}, 1 \mathrm{H}), 7.11-7.04(\mathrm{~m}, 2 \mathrm{H})$, $4.23-4.17(\mathrm{~m}, 2 \mathrm{H}), 3.72(\mathrm{~s}, 2 \mathrm{H}), 2.53-2.47(\mathrm{~m}, 2 \mathrm{H}), 2.04-1.97(\mathrm{~m}, 2 \mathrm{H}) .{ }^{13} \mathrm{C}$ NMR $(126 \mathrm{MHz}, \mathrm{CDCl} 3) \delta 210.12$, 157.39, 130.10, 129.94, 129.12, 124.60, 122.36, 75.75, 46.20, 39.90, 27.55. IR (neat, $\mathrm{cm}^{-1}$ ): 3058, 2960, 2892, 1702, $1581,1492,1256,1104,989,929,778,719 . \mathrm{ESI}^{+}$calculated for $\left[\mathrm{C}_{11} \mathrm{H}_{12} \mathrm{NaO}_{2}+\mathrm{MeOH}\right]^{+}: 231.10$ found: 231.08 . 


\section{Transformation of 3d to $5 H$-benzo[7]annulen-6(9H)-one (5)}

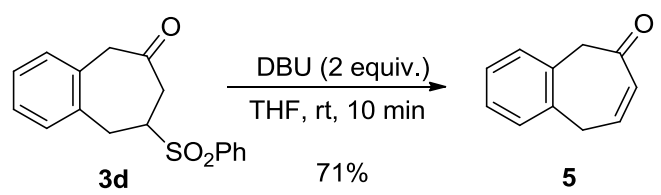

To a solution of $\mathbf{3 d}(0.1 \mathrm{mmol})$ in anhydrous THF $(2 \mathrm{ml})$ was added DBU (2.0 equiv.) in one portion. The resulting mixture was stirred for $10 \mathrm{~min}$. THF was then evaporated in vacuo, and the resulting residue was purified by silica gel flash chromatography (eluent: hexanes/ethyl acetate $=10 / 1$ ) to afford the desired product 5 in $71 \%$ yield. ${ }^{1} \mathrm{H}$ NMR $\left(500 \mathrm{MHz}, \mathrm{CDCl}_{3}\right) \delta 7.33-7.26(\mathrm{~m}, 3 \mathrm{H}), 7.22(\mathrm{~d}, J=7.2 \mathrm{~Hz}, 1 \mathrm{H}), 6.90(\mathrm{~d}, J=10.9 \mathrm{~Hz}, 1 \mathrm{H}), 6.05-5.96(\mathrm{~m}, 1 \mathrm{H}), 3.68$ (s, 2H), 3.07 (dd, $J=5.8,1.2 \mathrm{~Hz}, 2 \mathrm{H}) .{ }^{13} \mathrm{C}$ NMR $\left(125 \mathrm{MHz}, \mathrm{CDCl}_{3}\right) \delta 210.11,136.16,132.20,131.72,129.74,128.81$, 127.99, 126.97, 124.78, 50.80, 43.82. IR (neat, $\mathrm{cm}^{-1}$ ): 2918, 2850, 2270, 1635, 1576, 1466, 1266, 738. GC-MS: $\mathrm{m} / \mathrm{z}=$ $158\left[\mathrm{M}^{+}\right], 129,115,44$.

\section{References}

1. Caporusso, A. M.; Lardicci, L. J. Chem. Soc., Perkin Trans. 1, 1983, 949-953.

2. Allegretti, P. A.; Ferreira, E. M. Org. Lett. 2011, 13, 5924-5927.

3. Li, H.; Guillot, R.; Gandon, V. J. Org. Chem. 2010, 75, 8435-8449.

4. Stecko, S.; Mames, A.; Furman, B.; Chmielewski, M. J. Org. Chem. 2009, 74, 3094.

5. Handlon, A. L.; Guo, Y. Synlett. 2005, 111-114

6. Punna, S.; Meunier, S.; Finn, M. G. Org. Lett. 2004, 16, 2777-2779.

7. Xie, A. M.; Zhou, X. X; Feng, L. D.; Hu, X. Y.; Dong, W. Tetrahedron 2014, 70, 3514-3519. 


\begin{tabular}{|ll|}
\hline \multicolumn{1}{|c|}{ Parameter } & \multicolumn{1}{c|}{ Value } \\
1 Title & $\mathrm{xz}-1-248 \mathrm{~B} 500 \mathrm{H}$ \\
2 Solvent & $\mathrm{CDCl} 3$ \\
3 Spectrometer Frequency & 499.86 \\
4 Nucleus & $1 \mathrm{H}$ \\
\hline
\end{tabular}
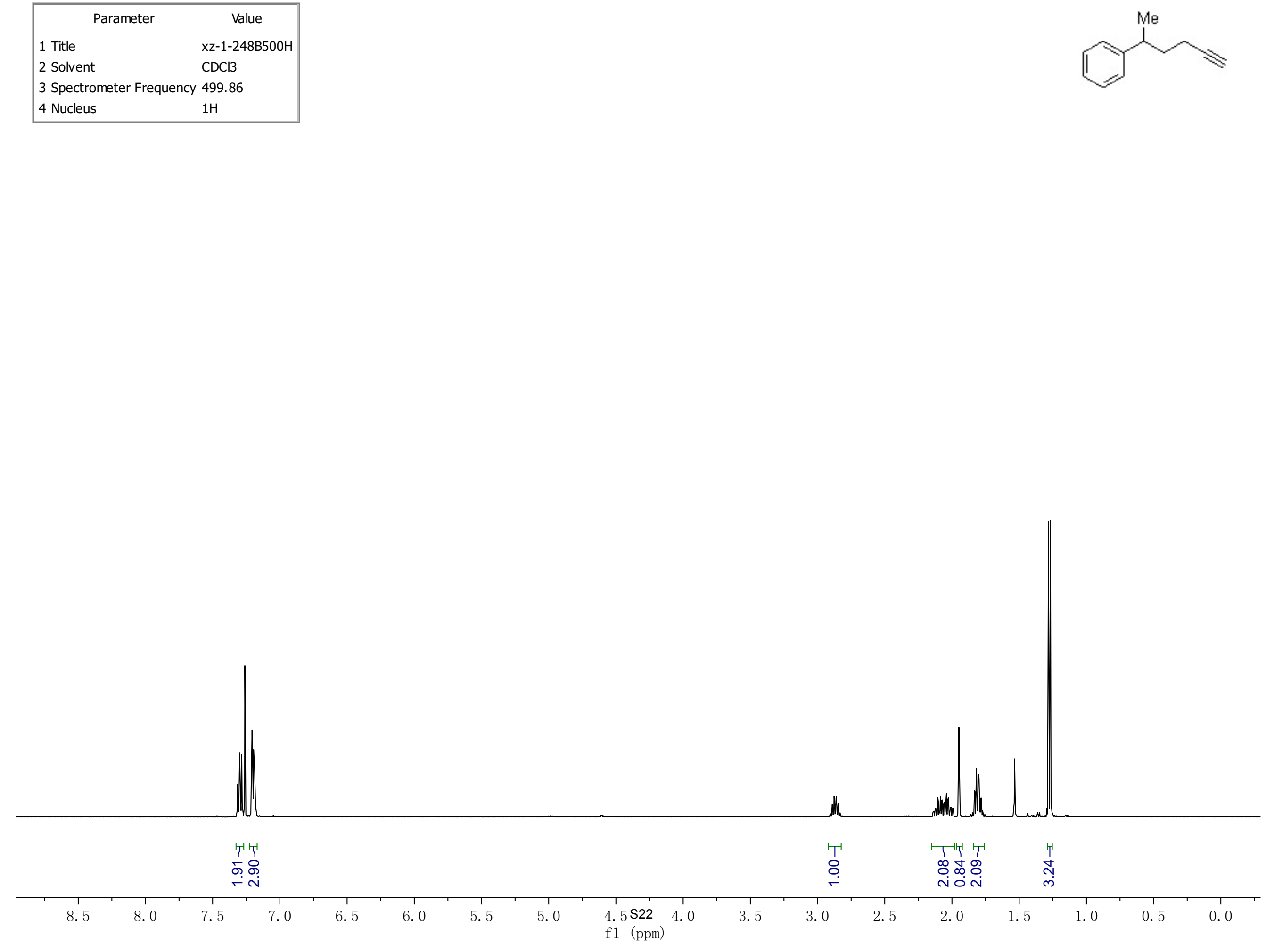


\begin{tabular}{|ll|}
\hline \multicolumn{1}{|c|}{ Parameter } & \multicolumn{1}{c|}{ Value } \\
1 Title & $\mathrm{xz}-1-248 \mathrm{~B} 500 \mathrm{C}$ \\
2 Solvent & $\mathrm{CDCl3}$ \\
3 Spectrometer Frequency & 125.70 \\
4 Nucleus & $13 \mathrm{C}$ \\
\hline
\end{tabular}
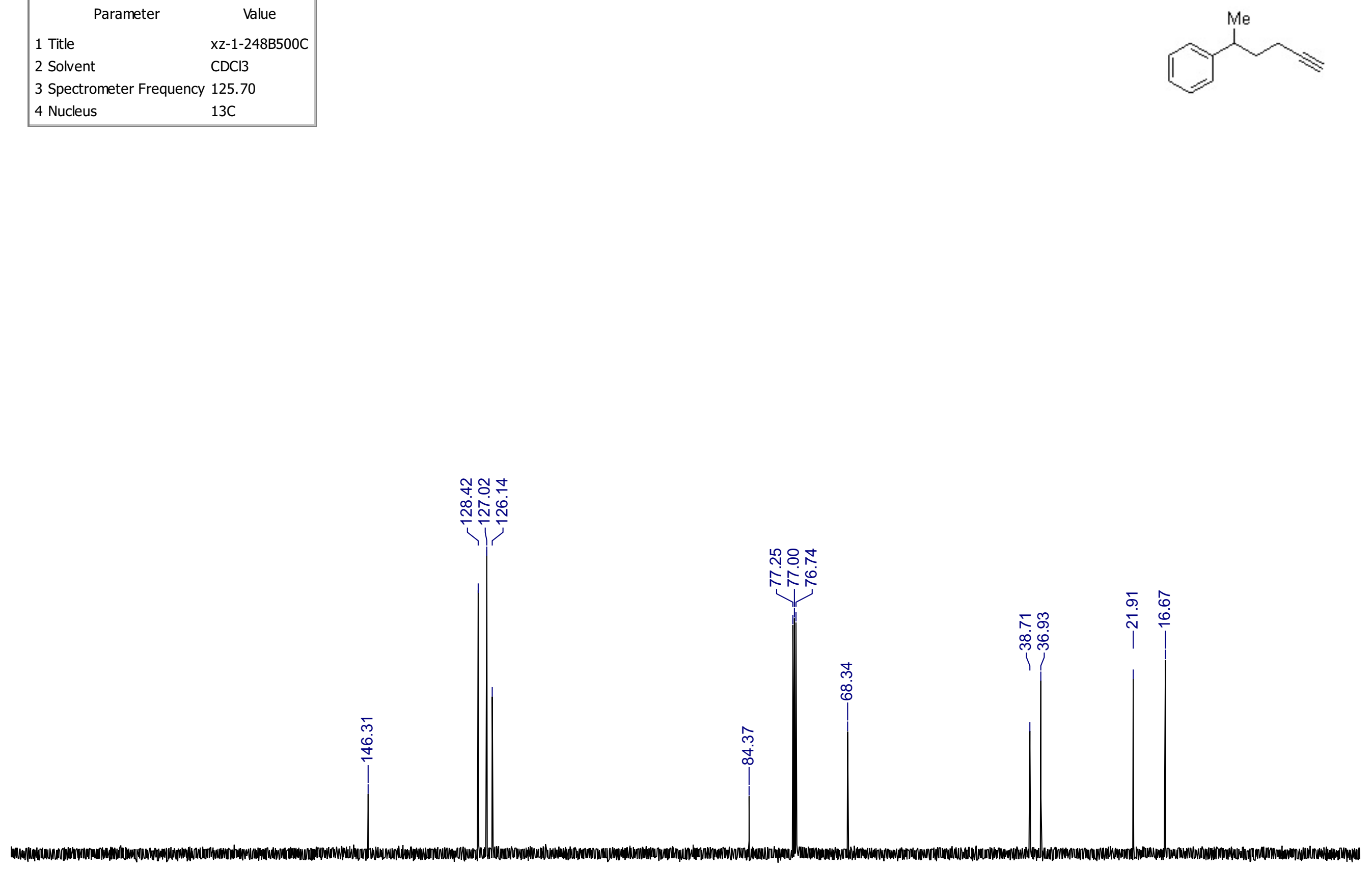

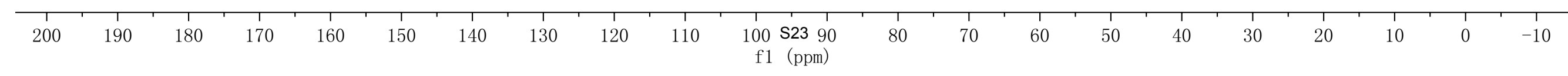




\begin{tabular}{|ll|}
\hline \multicolumn{1}{|c|}{ Parameter } & \multicolumn{1}{c|}{ Value } \\
1 Title & xz-1-189D2 \\
2 Solvent & $c d c 13$ \\
3 Spectrometer Frequency & 399.78 \\
4 Nucleus & $1 \mathrm{H}$ \\
\hline
\end{tabular}
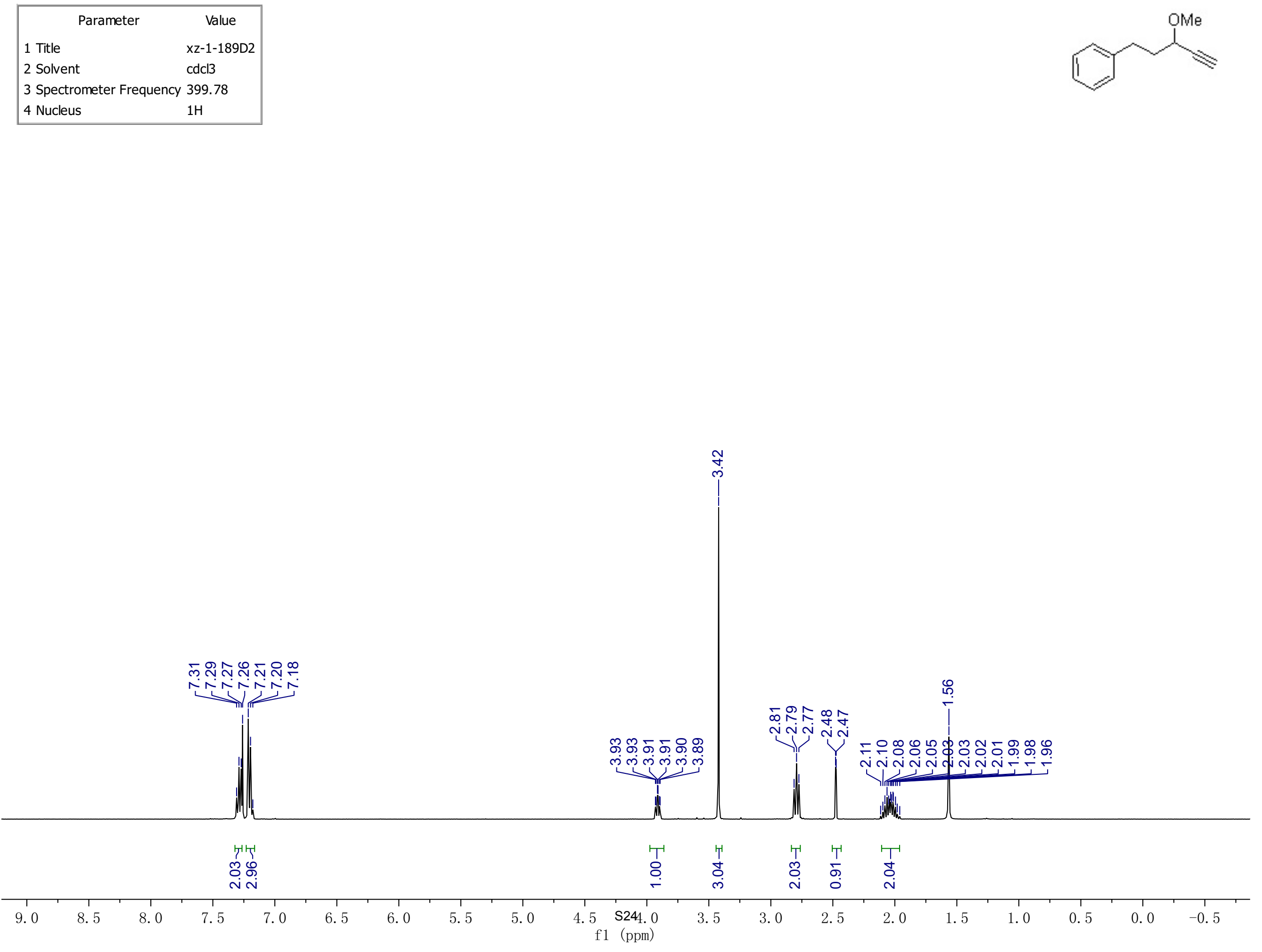


\begin{tabular}{|ll|}
\hline \multicolumn{1}{|c|}{ Parameter } & \multicolumn{1}{c|}{ Value } \\
1 Title & $\mathrm{xz}-1-189 \mathrm{D} 3$ C500biaopu \\
2 Solvent & $\mathrm{CDCl} 3$ \\
3 Spectrometer Frequency & 125.70 \\
4 Nucleus & $13 \mathrm{C}$ \\
\hline
\end{tabular}
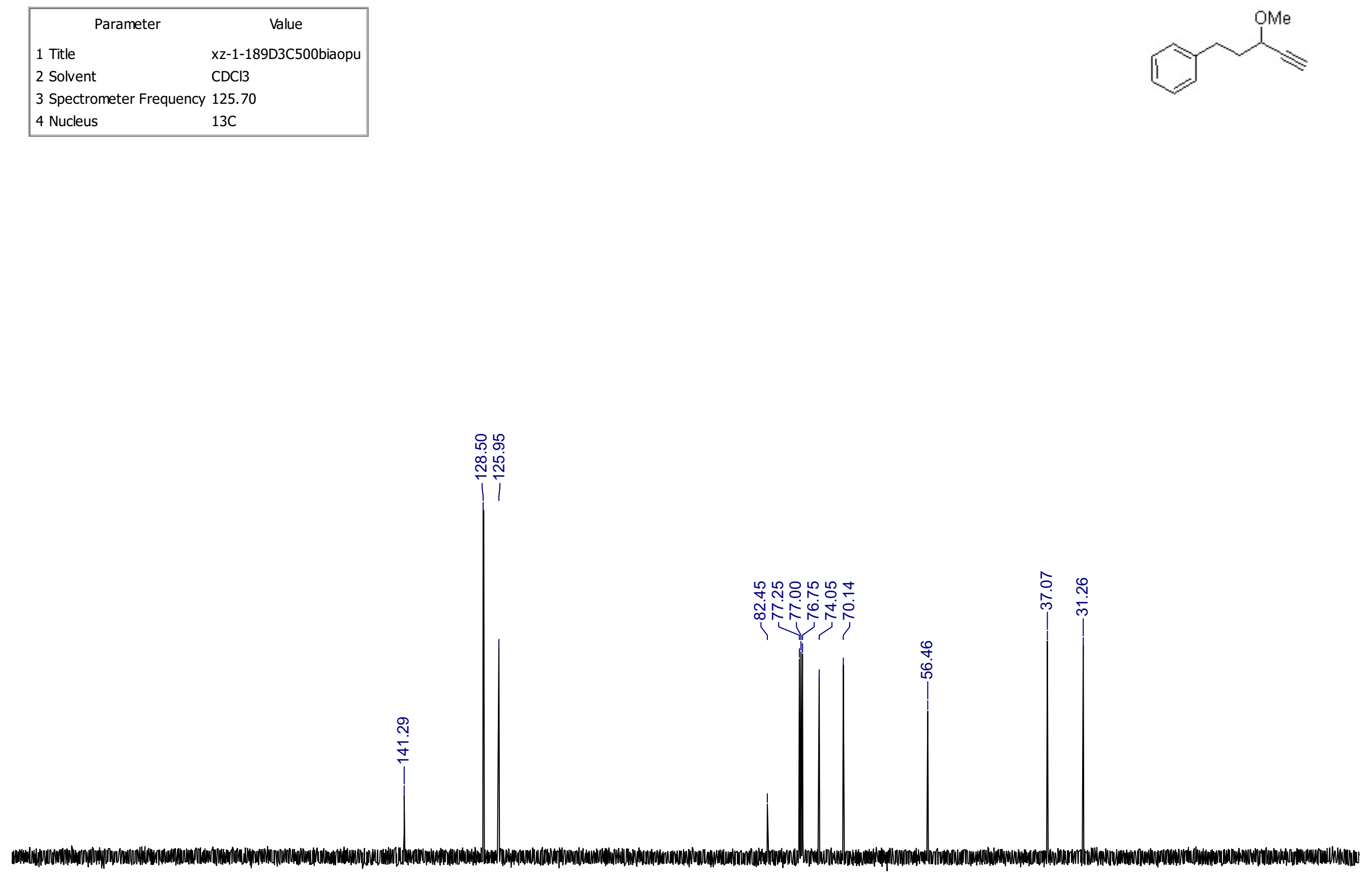

\begin{tabular}{|c|c|c|c|c|c|c|c|c|c|c|c|c|c|c|c|c|c|c|c|c|}
\hline 1 & 1 & 1 & 1 & 1 & 1 & 1 & 1 & 1 & 1 & $\begin{array}{llll} & 1 & 1\end{array}$ & 1 & 1 & 1 & 1 & 1 & 1 & 1 & 1 & $T$ & 1 \\
\hline 200 & 190 & 180 & 170 & 160 & 150 & 140 & 130 & 120 & 110 & $\begin{array}{l}100 S 2590 \\
\text { f1 (ppm) }\end{array}$ & 80 & 70 & 60 & 50 & 40 & 30 & 20 & 10 & 0 & -10 \\
\hline
\end{tabular}




\begin{tabular}{|ll|}
\hline \multicolumn{1}{|c}{ Parameter } & \multicolumn{1}{c|}{ Value } \\
1 Title & xz-1-201cbiaopu \\
2 Solvent & $c d c l 3$ \\
3 Spectrometer Frequency & 399.78 \\
4 Nucleus & $1 \mathrm{H}$ \\
\hline
\end{tabular}
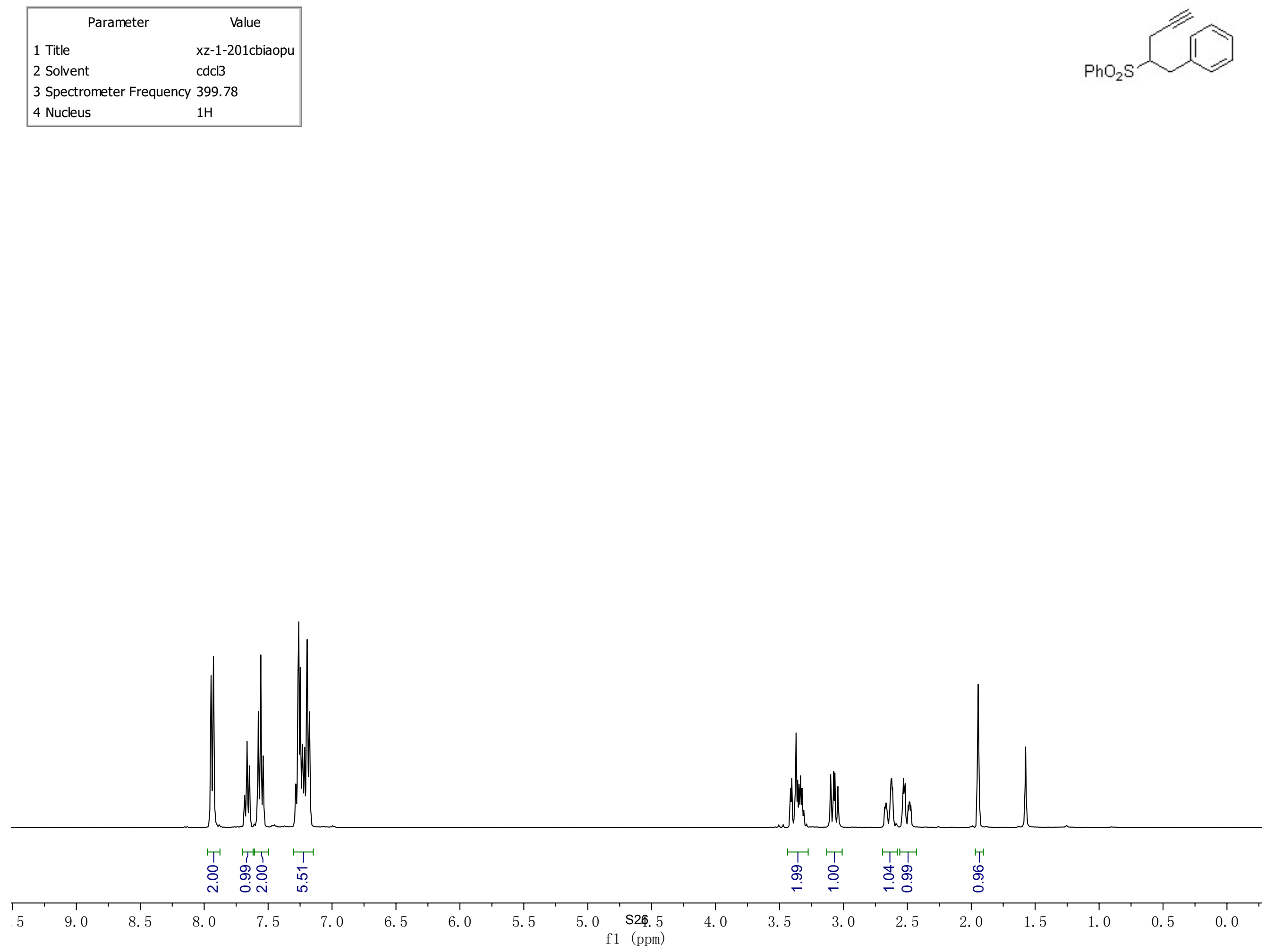


\begin{tabular}{|ll|}
\hline \multicolumn{1}{|c|}{ Parameter } & \multicolumn{1}{c|}{ Value } \\
1 Title & $\mathrm{xz}-1-201 \mathrm{fCbiaopu} 500$ \\
2 Solvent & $\mathrm{CDCl3}$ \\
3 Spectrometer Frequency & 125.70 \\
4 Nucleus & $13 \mathrm{C}$ \\
\hline
\end{tabular}

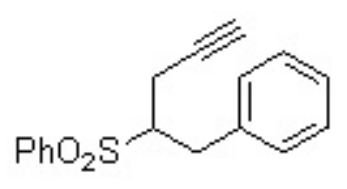

ชூํำ

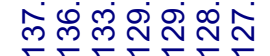
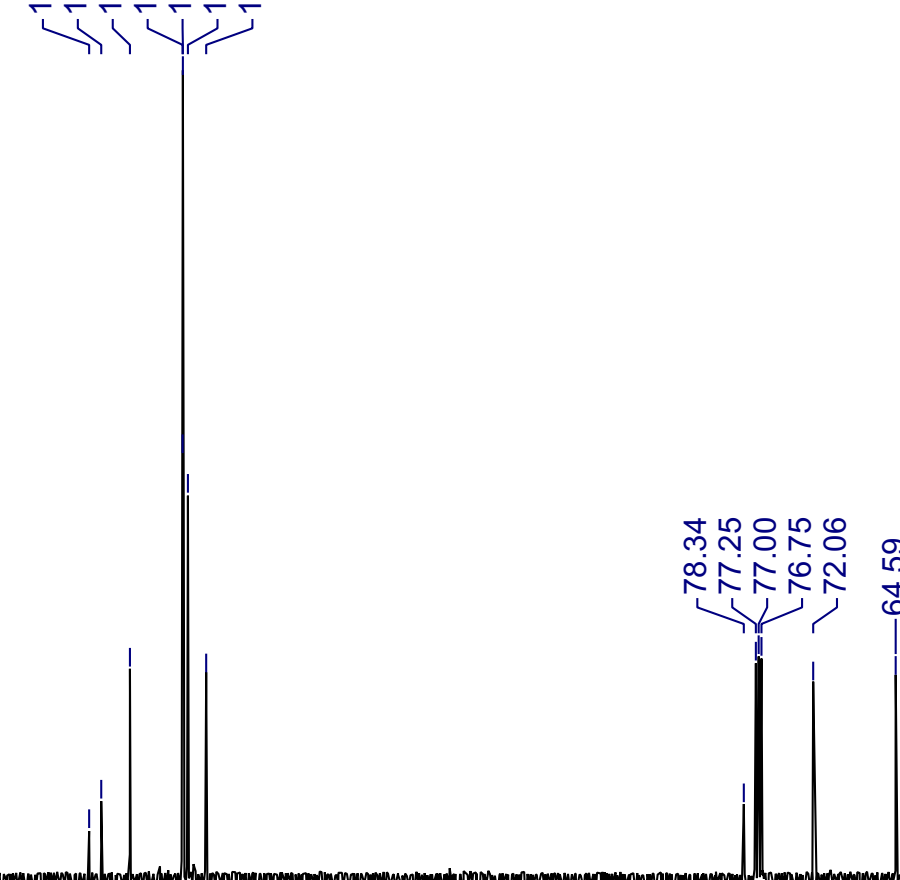

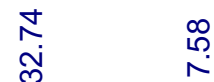

$\stackrel{\stackrel{\leftrightarrow}{\Gamma}}{\Gamma}$

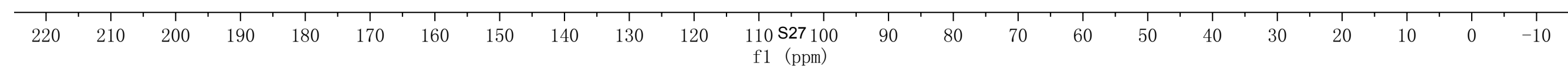




\begin{tabular}{|ll|}
\hline \multicolumn{1}{|c}{ Parameter } & \multicolumn{1}{c|}{ Value } \\
1 Title & xz-1-201dbiaopu \\
2 Solvent & cdcl3 \\
3 Spectrometer Frequency & 399.78 \\
4 Nucleus & $1 \mathrm{H}$ \\
\hline
\end{tabular}
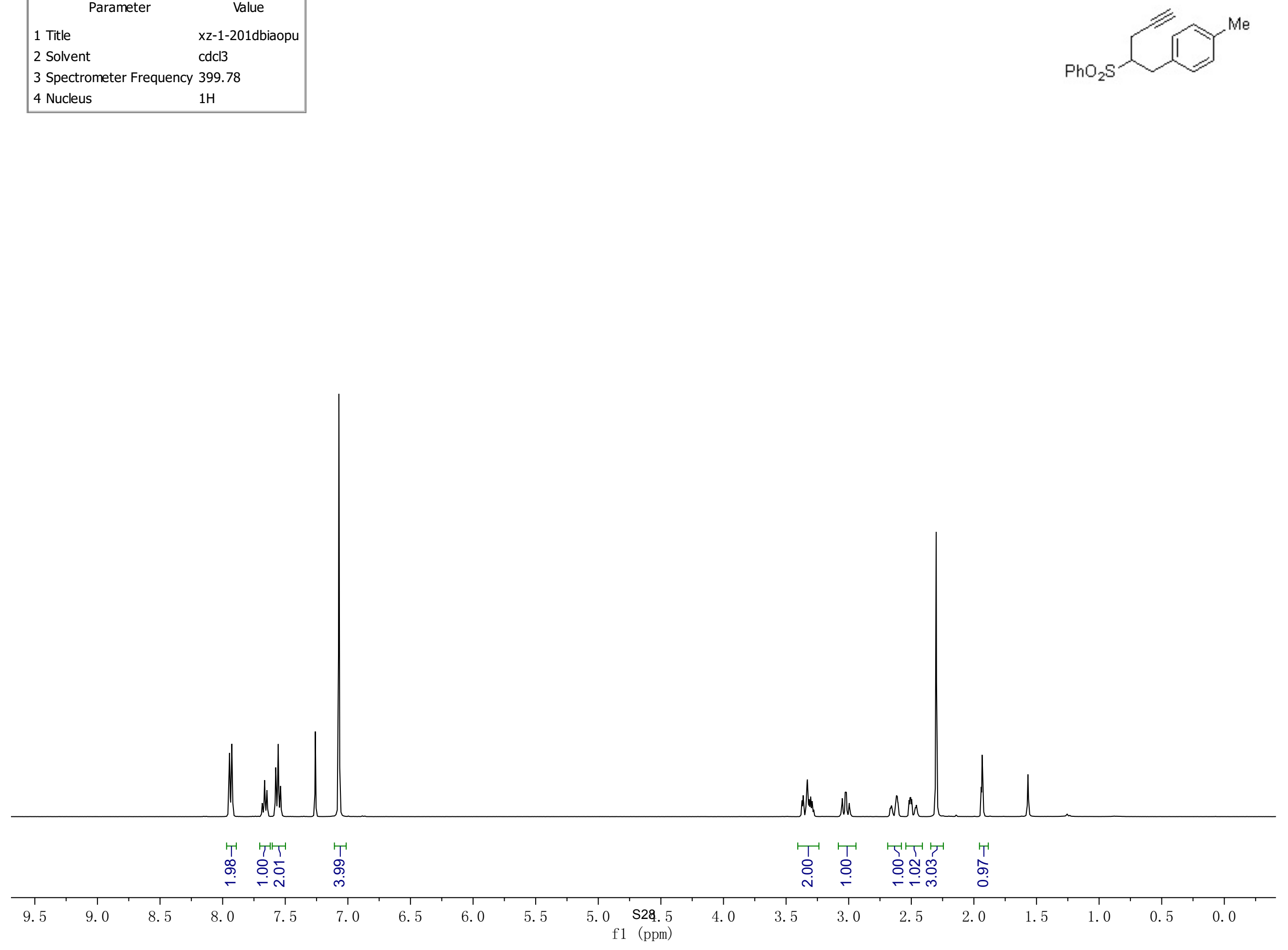


\begin{tabular}{|ll|}
\hline \multicolumn{1}{|c|}{ Parameter } & \multicolumn{1}{c|}{ Value } \\
1 Title & $\mathrm{xz}-1-201 \mathrm{dCb}$ Ciaopu500 \\
2 Solvent & $\mathrm{CDCl} 3$ \\
3 Spectrometer Frequency & 125.70 \\
4 Nucleus & $13 \mathrm{C}$ \\
\hline
\end{tabular}

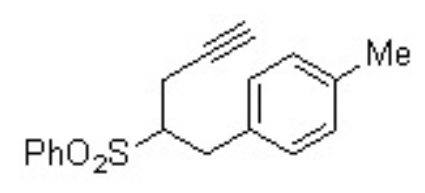

๑

侘

-
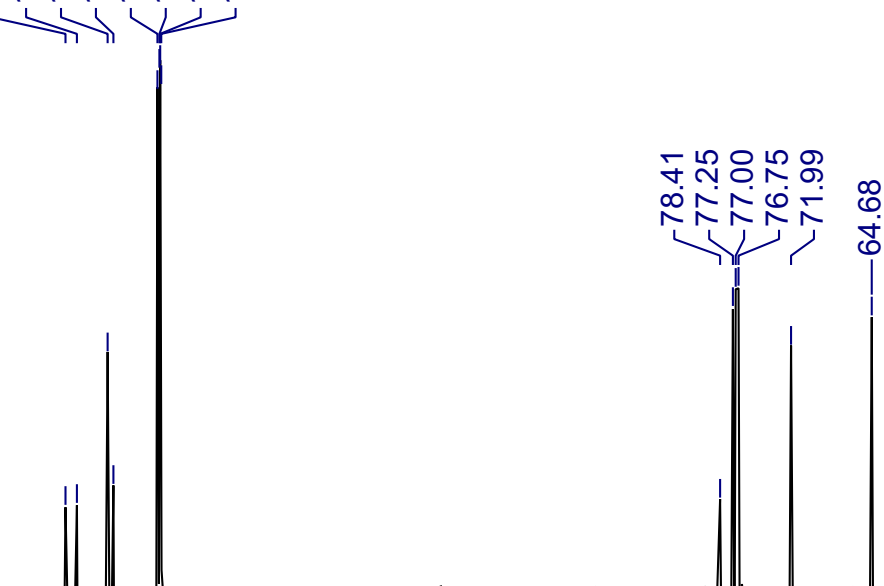

సิ $\quad \bar{c}$

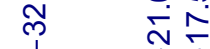

$\stackrel{\text { I }}{1}$

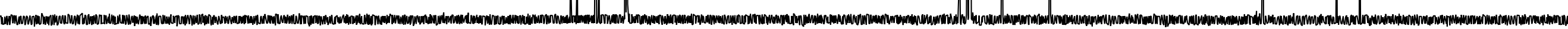

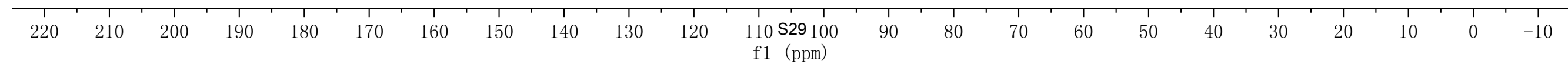




\begin{tabular}{|ll|}
\hline \multicolumn{1}{|c|}{ Parameter } & \multicolumn{1}{c|}{ Value } \\
1 Title & $\mathrm{xz}-1-201 \mathrm{fbiaopu}$ \\
2 Solvent & $\mathrm{cdcl} 3$ \\
3 Spectrometer Frequency & 399.78 \\
4 Nucleus & $1 \mathrm{H}$ \\
\hline
\end{tabular}
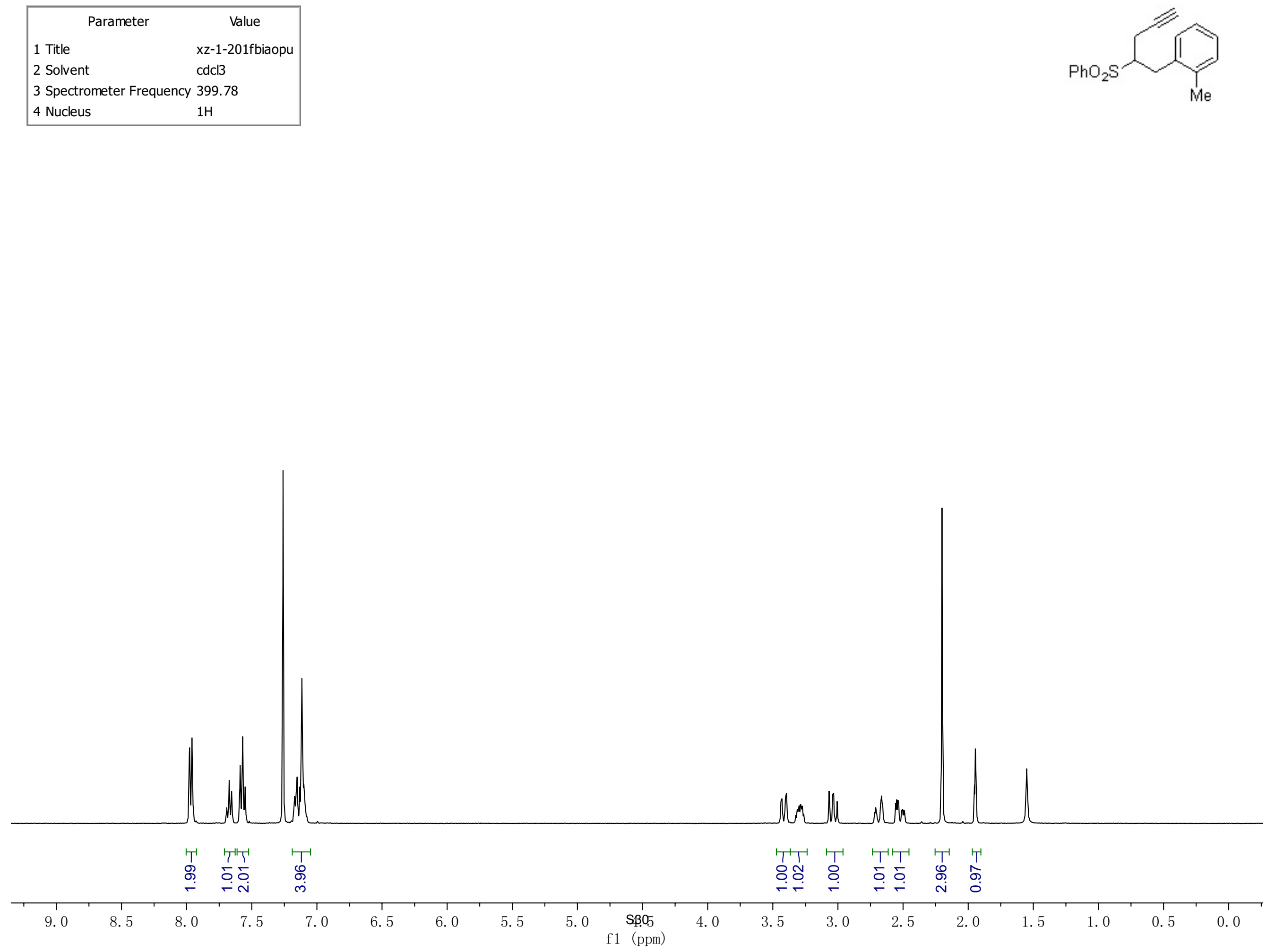


\begin{tabular}{|ll|}
\hline \multicolumn{1}{|c|}{ Parameter } & \multicolumn{1}{c|}{ Value } \\
1 Title & $\mathrm{xz}-1-201 \mathrm{fbiaoc} 500$ \\
2 Solvent & $\mathrm{CDCl} 3$ \\
3 Spectrometer Frequency & 125.70 \\
4 Nucleus & $13 \mathrm{C}$ \\
\hline
\end{tabular}

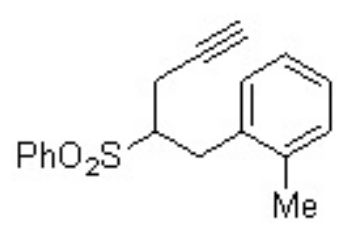

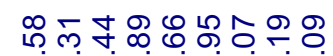

mím

员

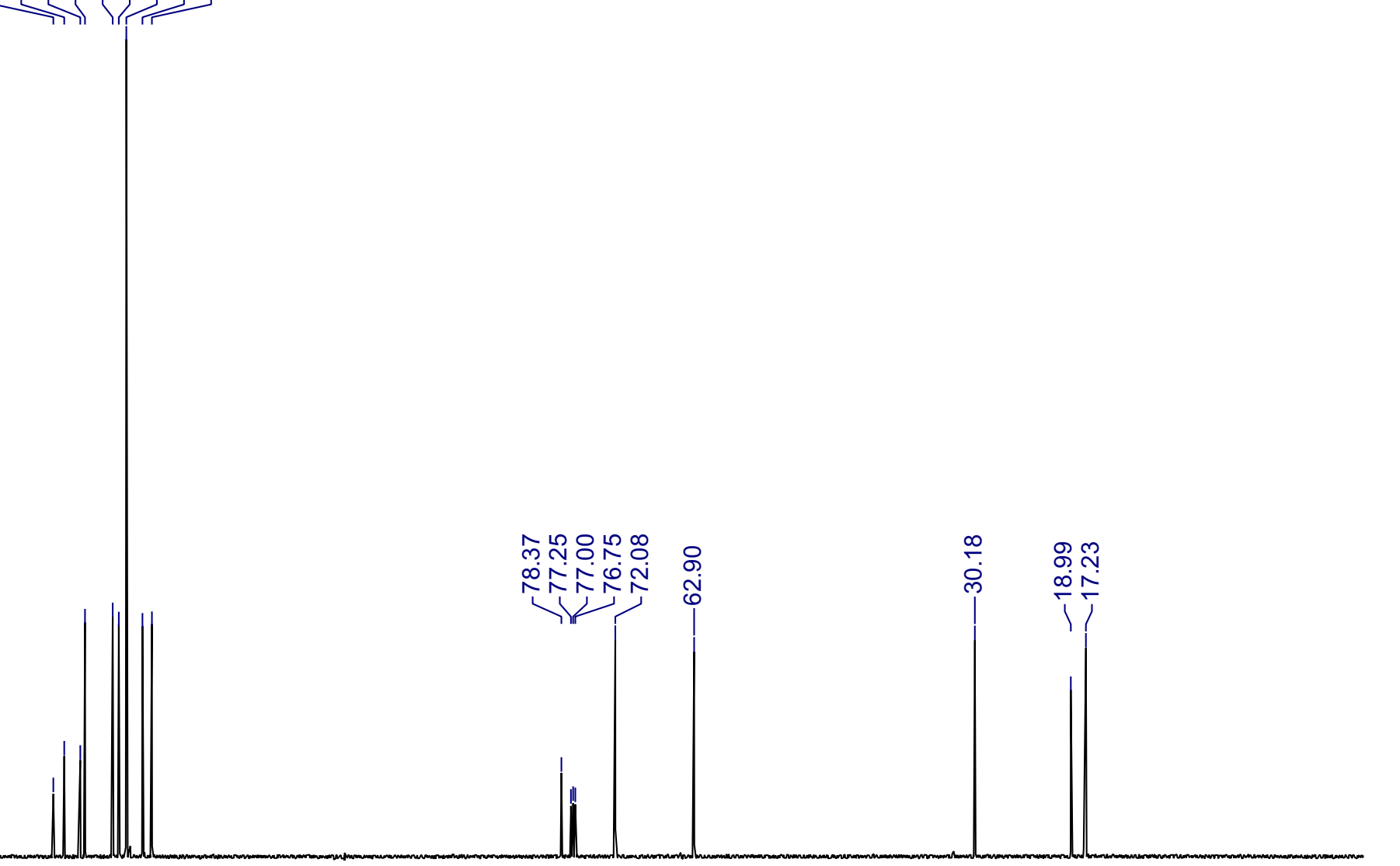

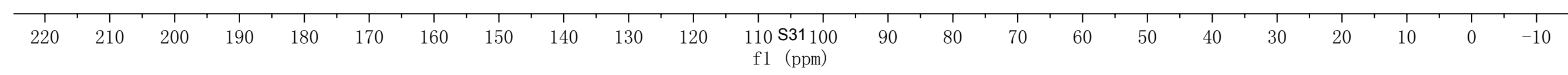




\begin{tabular}{|ll|}
\hline \multicolumn{1}{|c|}{ Parameter } & \multicolumn{1}{c|}{ Value } \\
1 Title & $\mathrm{xz}-1-237 \mathrm{Bbiaopu} 500$ \\
2 Solvent & $\mathrm{CDCl3}$ \\
3 Spectrometer Frequency & 499.86 \\
4 Nucleus & $1 \mathrm{H}$ \\
\hline
\end{tabular}
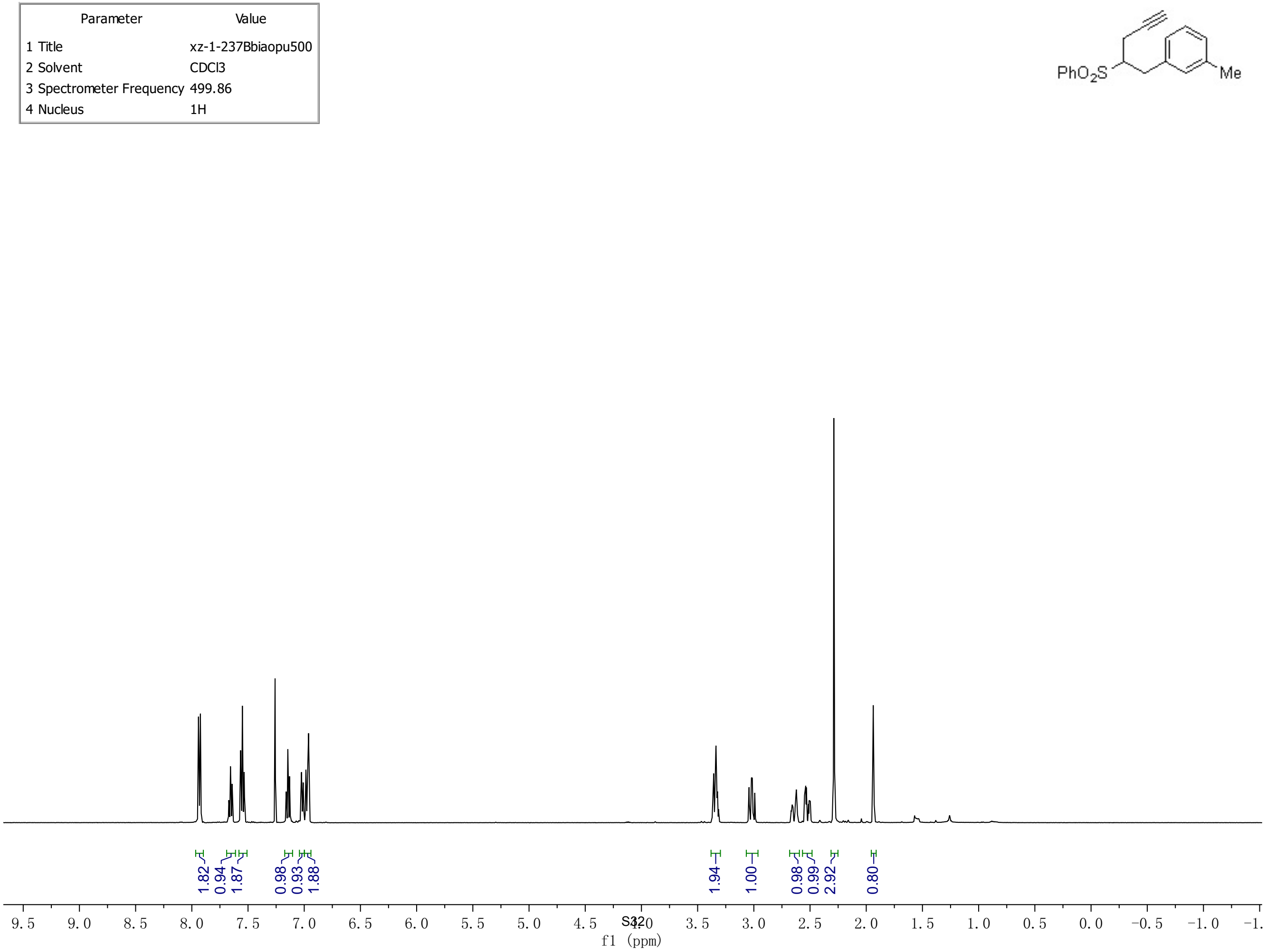


\begin{tabular}{|ll|}
\hline \multicolumn{1}{|c|}{ Parameter } & \multicolumn{1}{c|}{ Value } \\
1 Title & $\mathrm{xz}-1-237$ Bbiaopu500 \\
2 Solvent & $\mathrm{CDCl} 3$ \\
3 Spectrometer Frequency & 499.86 \\
4 Nucleus & $1 \mathrm{H}$ \\
\hline
\end{tabular}

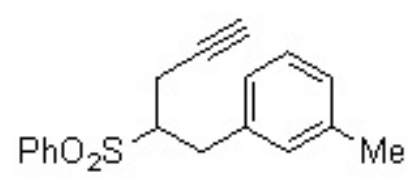

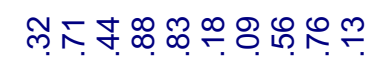
m.

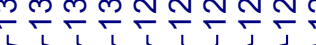
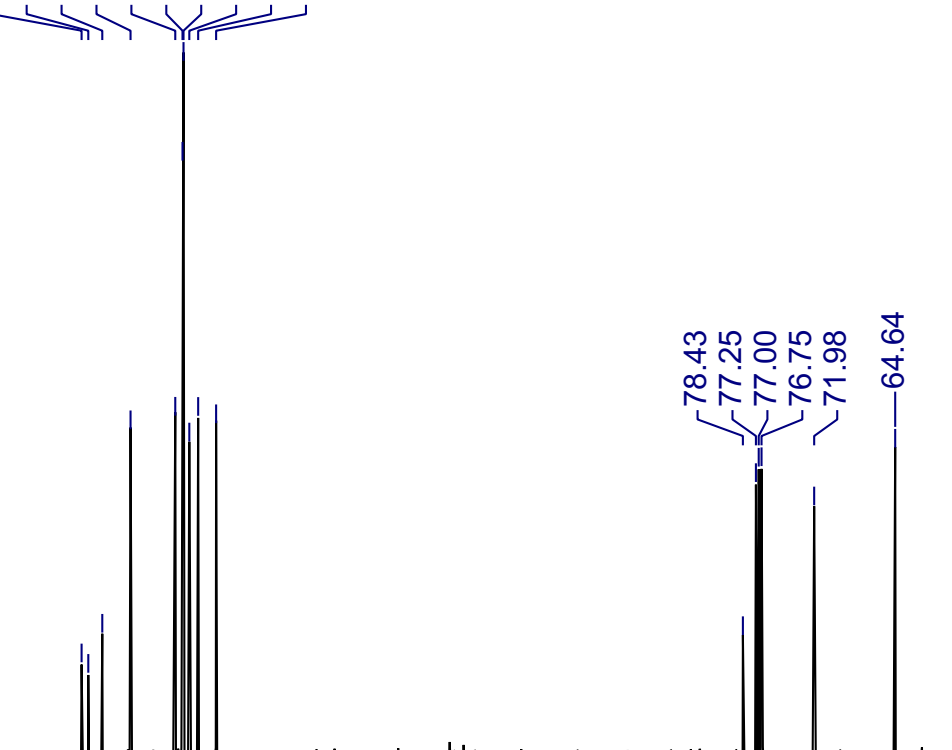

๕ ๓

ก্ল

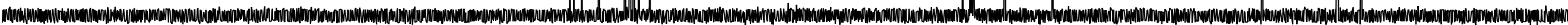

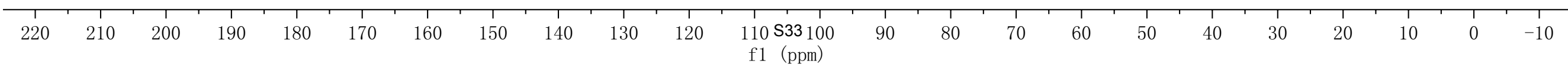




\begin{tabular}{|ll|}
\hline \multicolumn{1}{|c|}{ Parameter } & \multicolumn{1}{c|}{ Value } \\
1 Title & $\mathrm{xz}-1-214 \mathrm{~A}$ \\
2 Solvent & $\mathrm{CDCl} 3$ \\
3 Spectrometer Frequency & 499.86 \\
4 Nucleus & $1 \mathrm{H}$ \\
\hline
\end{tabular}
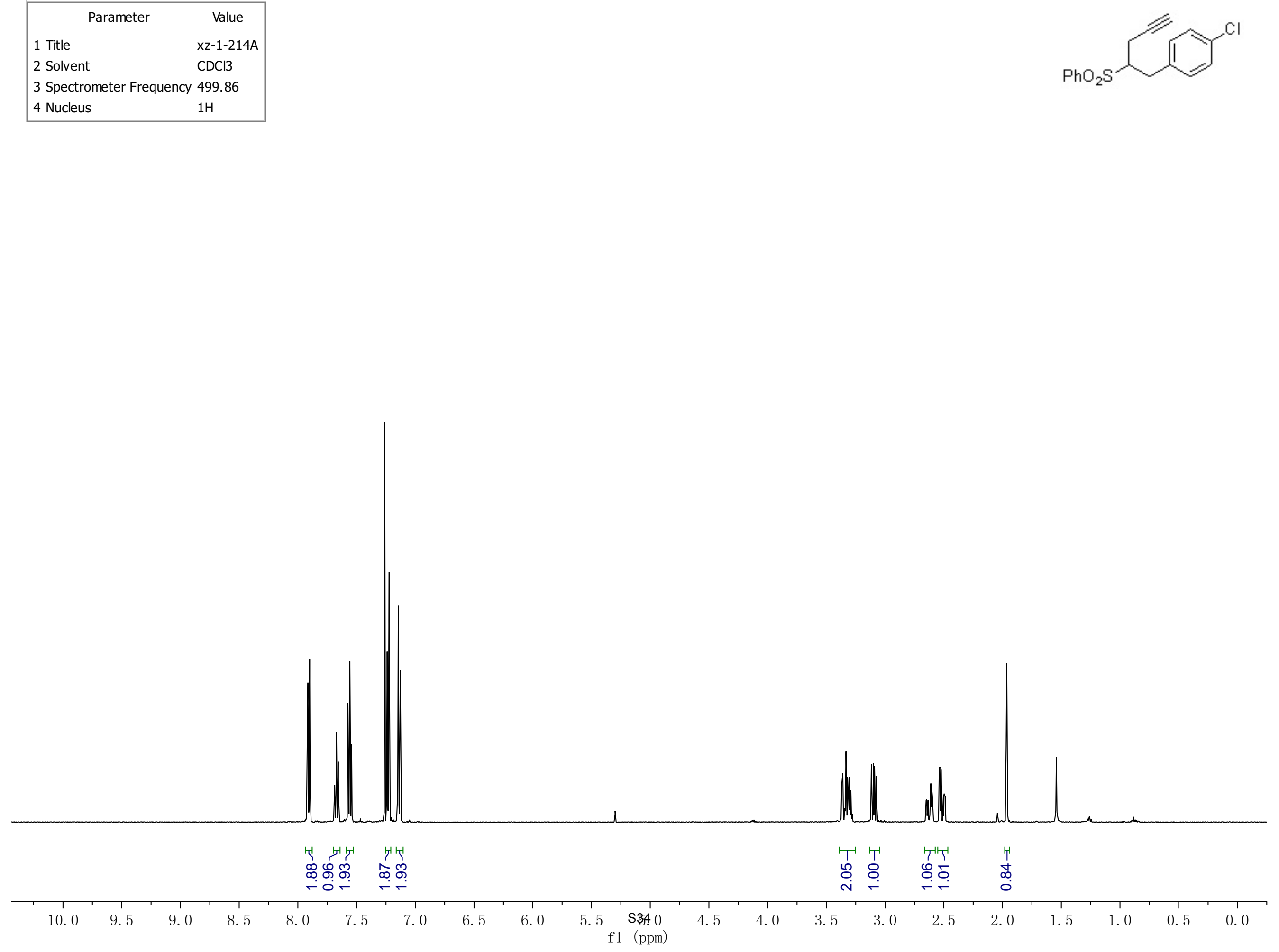


\begin{tabular}{|ll|}
\hline \multicolumn{1}{|c|}{ Parameter } & \multicolumn{1}{c|}{ Value } \\
1 Title & $\mathrm{xz}-1-214 \mathrm{AC} 500$ \\
2 Solvent & $\mathrm{CDCl} 3$ \\
3 Spectrometer Frequency & 125.70 \\
4 Nucleus & $13 \mathrm{C}$ \\
\hline
\end{tabular}

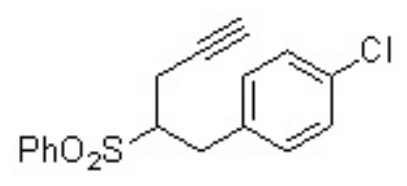

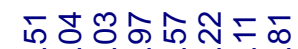
रें nं के के mำ m

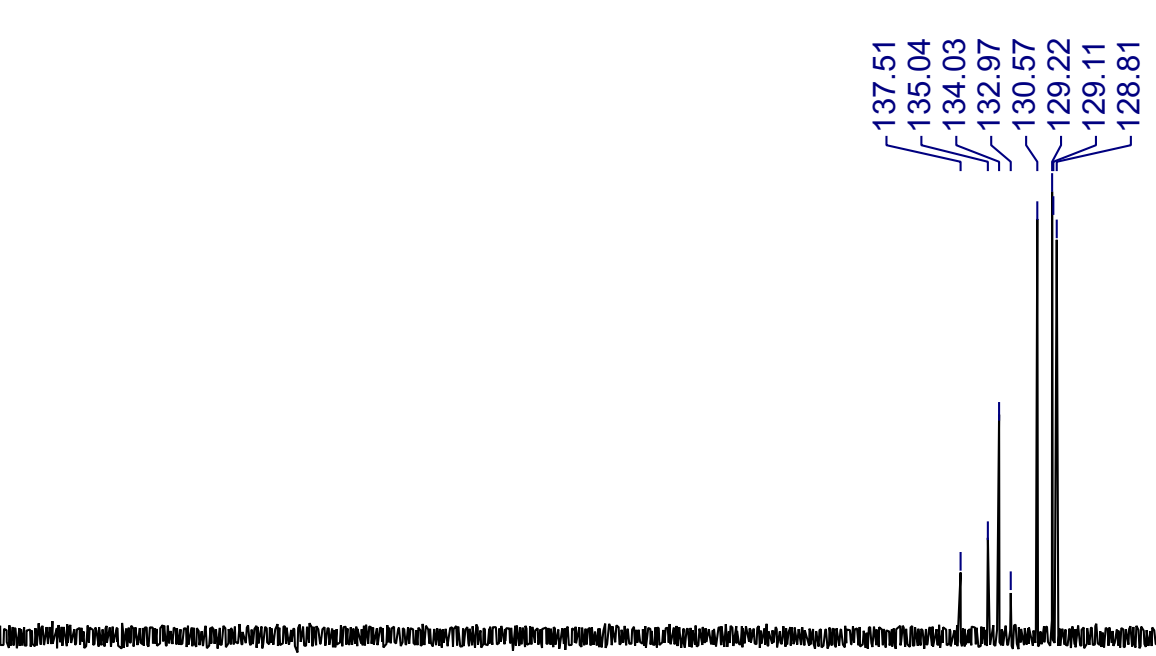

กฺุุำำำ

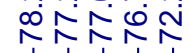

金亦
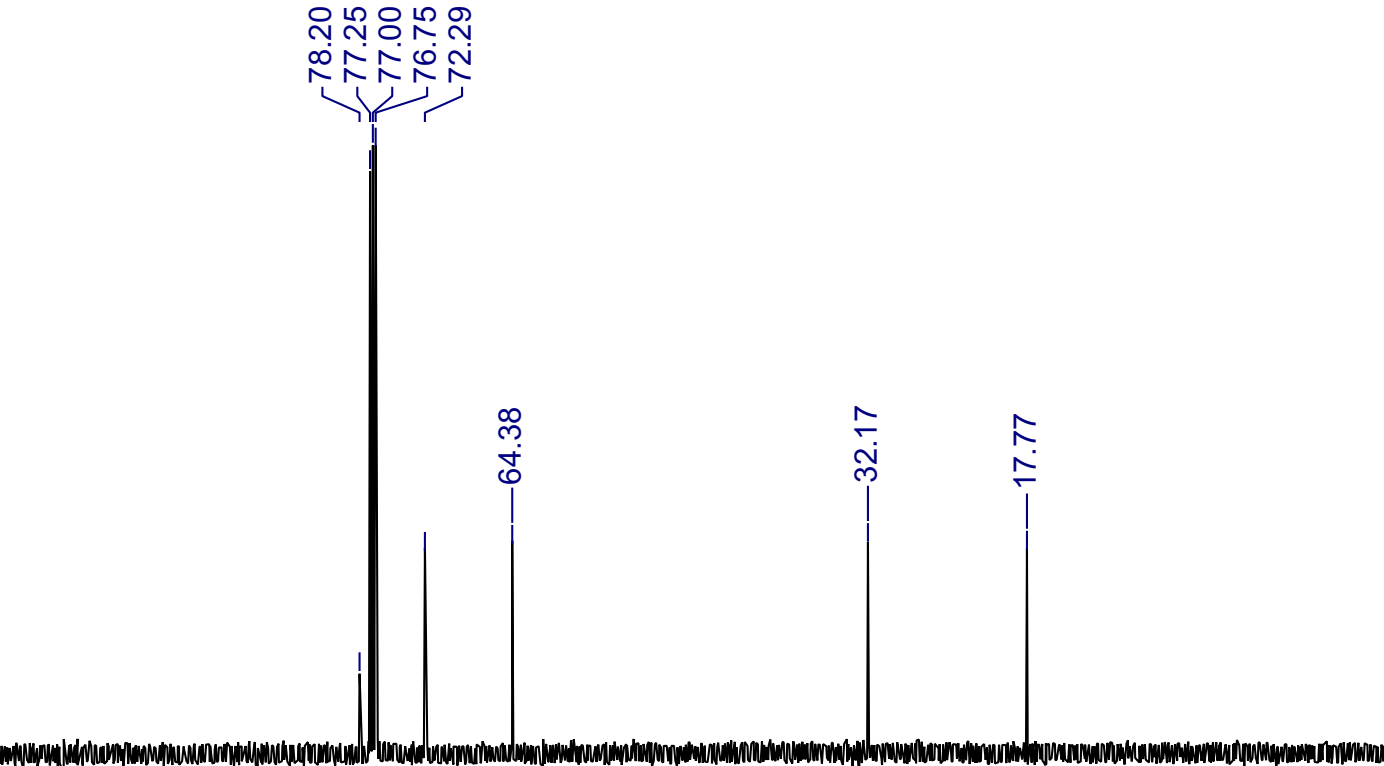


\begin{tabular}{|ll|}
\hline \multicolumn{1}{|c|}{ Parameter } & \multicolumn{1}{c|}{ Value } \\
1 Title & $\mathrm{xz}-1-260 \mathrm{H}$ \\
2 Solvent & $\mathrm{cdcl} 3$ \\
3 Spectrometer Frequency & 399.78 \\
4 Nucleus & $1 \mathrm{H}$ \\
\hline
\end{tabular}
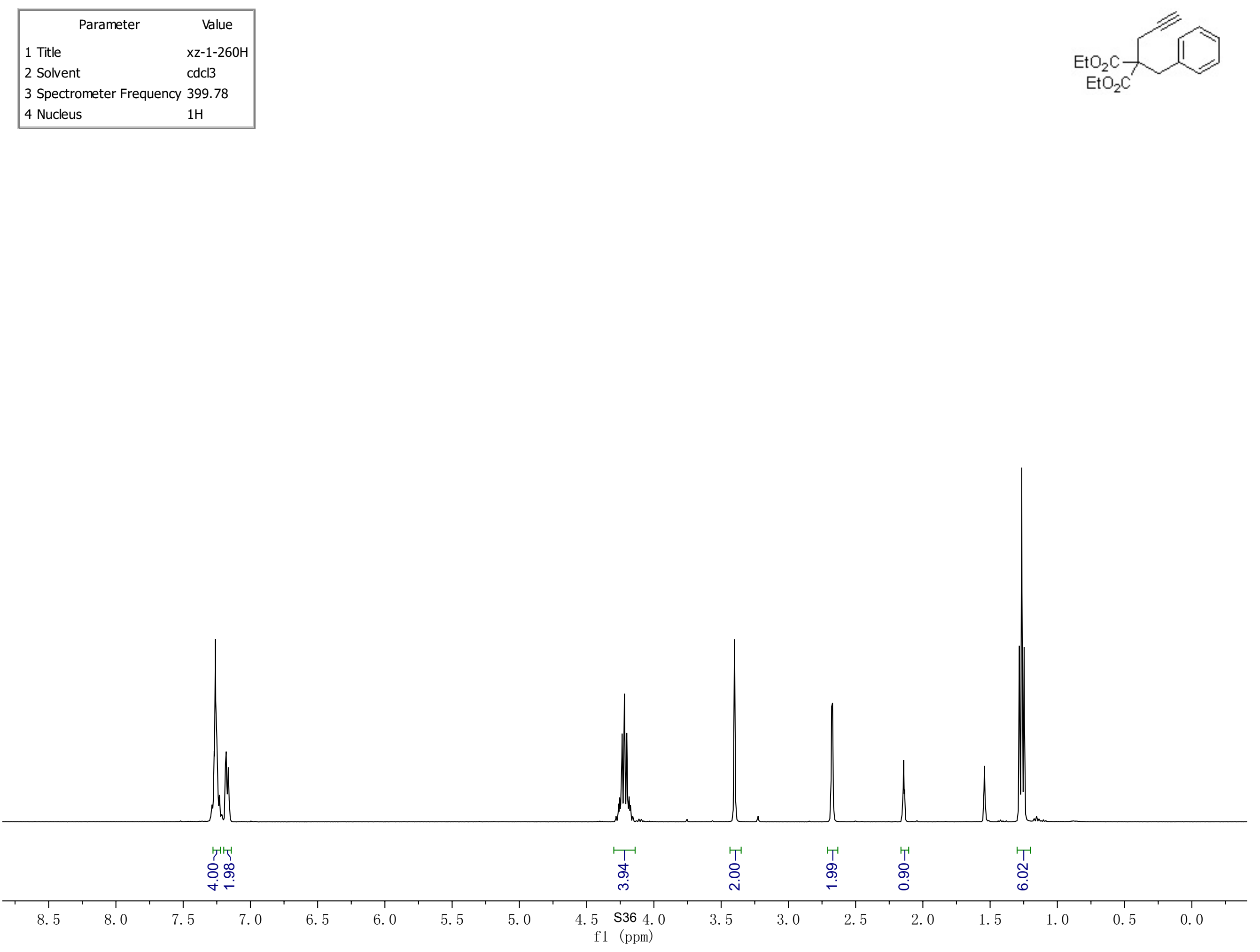


\begin{tabular}{|ll|}
\hline \multicolumn{1}{|c|}{ Parameter } & \multicolumn{1}{c|}{ Value } \\
1 Title & $\mathrm{xz}-1-260 \mathrm{C}$ \\
2 Solvent & $\mathrm{CDCl} 3$ \\
3 Spectrometer Frequency & 125.70 \\
4 Nucleus & $13 \mathrm{C}$ \\
\hline
\end{tabular}

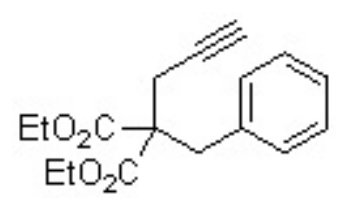

œ

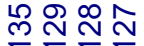



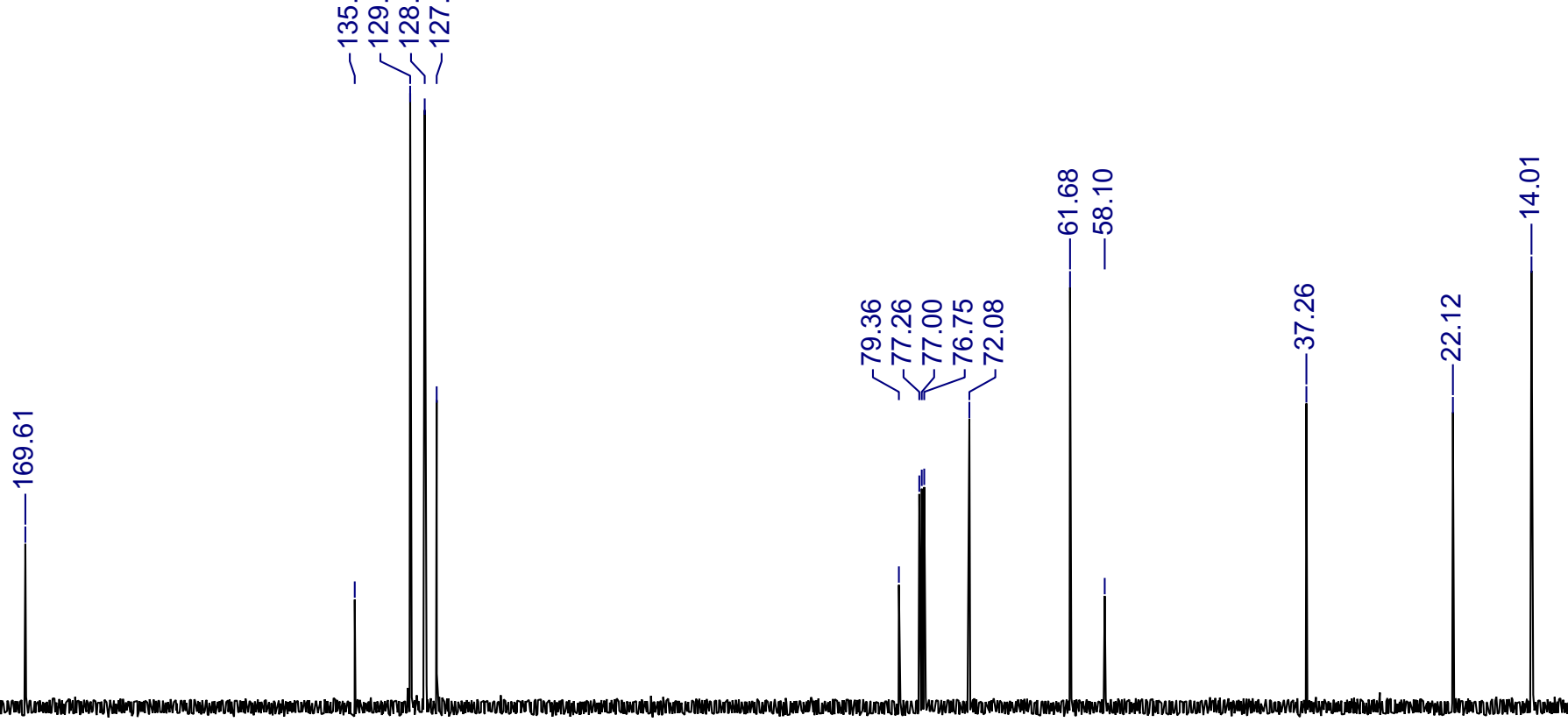

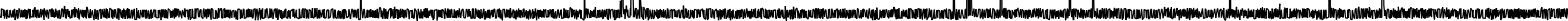

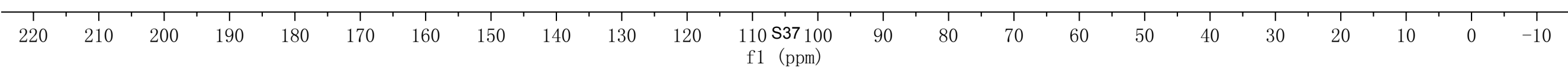




\begin{tabular}{|c|c|}
\hline Parameter & Value \\
\hline 1 Title & xz-1-261BHbiaopu500 \\
\hline 2 Solvent & $\mathrm{CDCl} 3$ \\
\hline \multicolumn{2}{|c|}{3 Spectrometer Frequency 499.86} \\
\hline 4 Nucleus & $1 \mathrm{H}$ \\
\hline
\end{tabular}

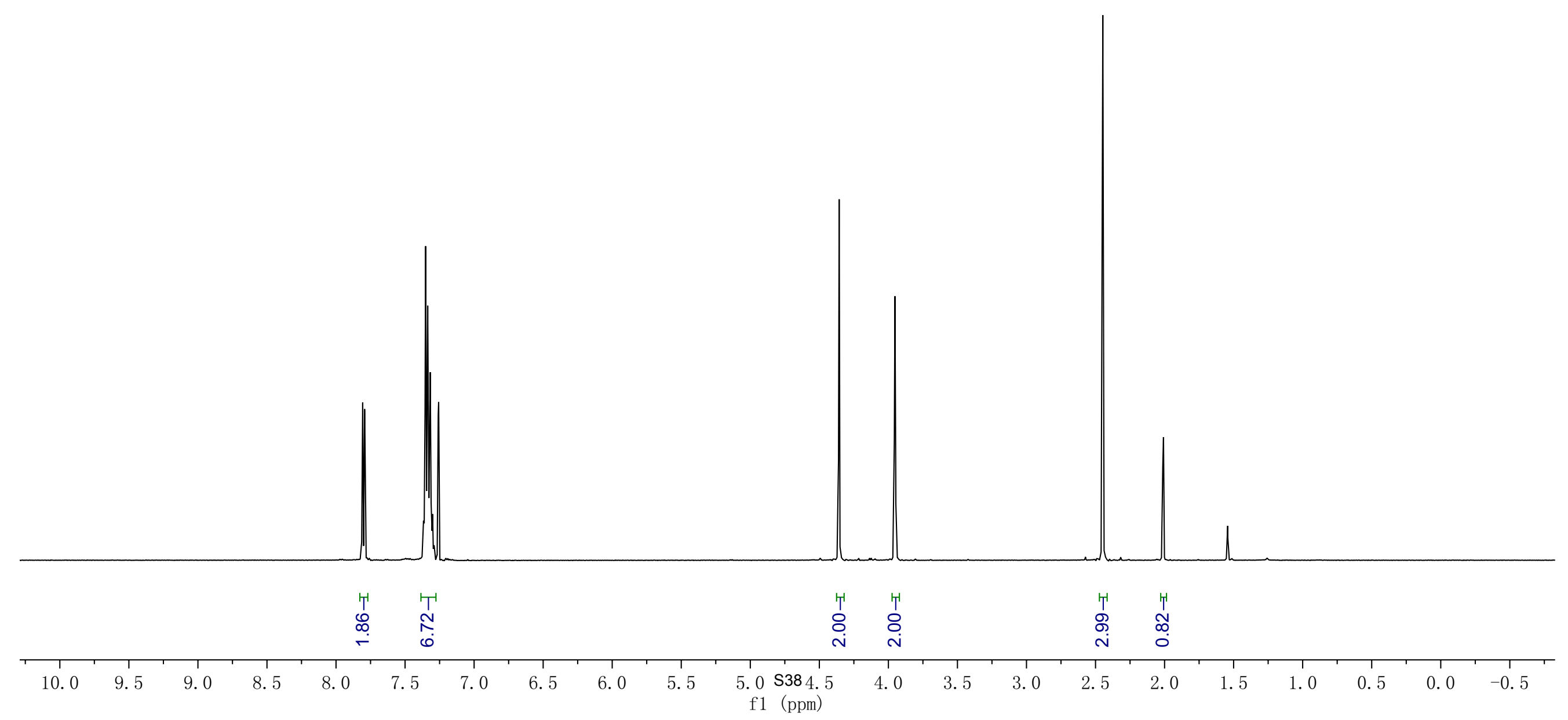




\begin{tabular}{|ll|}
\hline \multicolumn{1}{|c|}{ Parameter } & \multicolumn{1}{c|}{ Value } \\
1 Title & $\mathrm{xz}-1-261 \mathrm{BCbiaopu500}$ \\
2 Solvent & $\mathrm{CDCl3}$ \\
3 Spectrometer Frequency & 125.70 \\
4 Nucleus & $13 \mathrm{C}$ \\
\hline
\end{tabular}
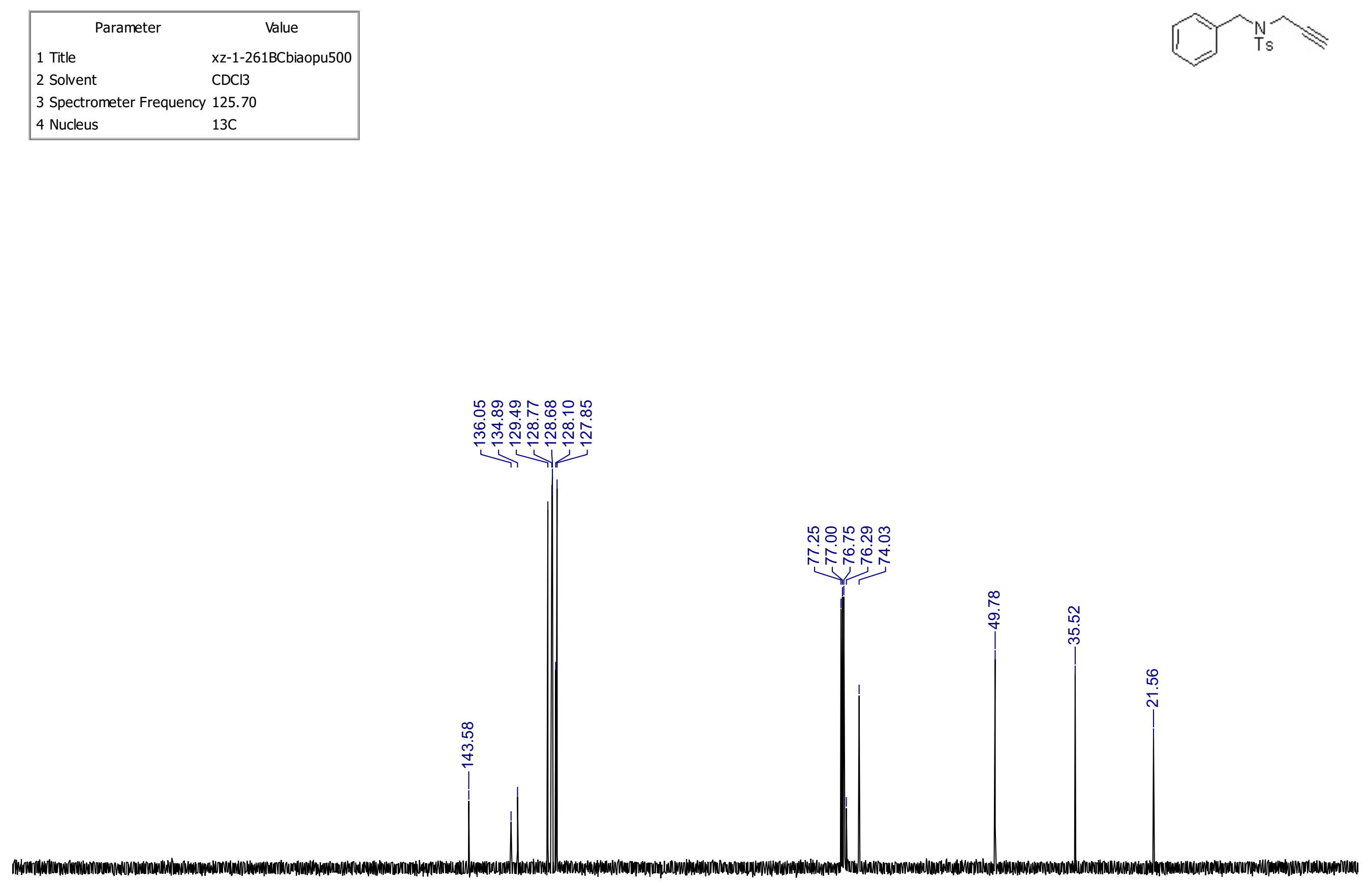

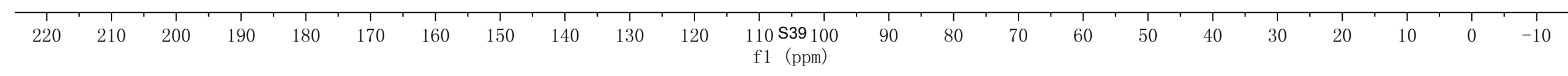




\begin{tabular}{|ll|}
\hline \multicolumn{1}{|c|}{ Parameter } & \multicolumn{1}{c|}{ Value } \\
1 Title & $\mathrm{xz}-1-216 \mathrm{Bbiaopu}$ \\
2 Solvent & $\mathrm{CDCl} 3$ \\
3 Spectrometer Frequency & 499.86 \\
4 Nucleus & $1 \mathrm{H}$ \\
\hline
\end{tabular}
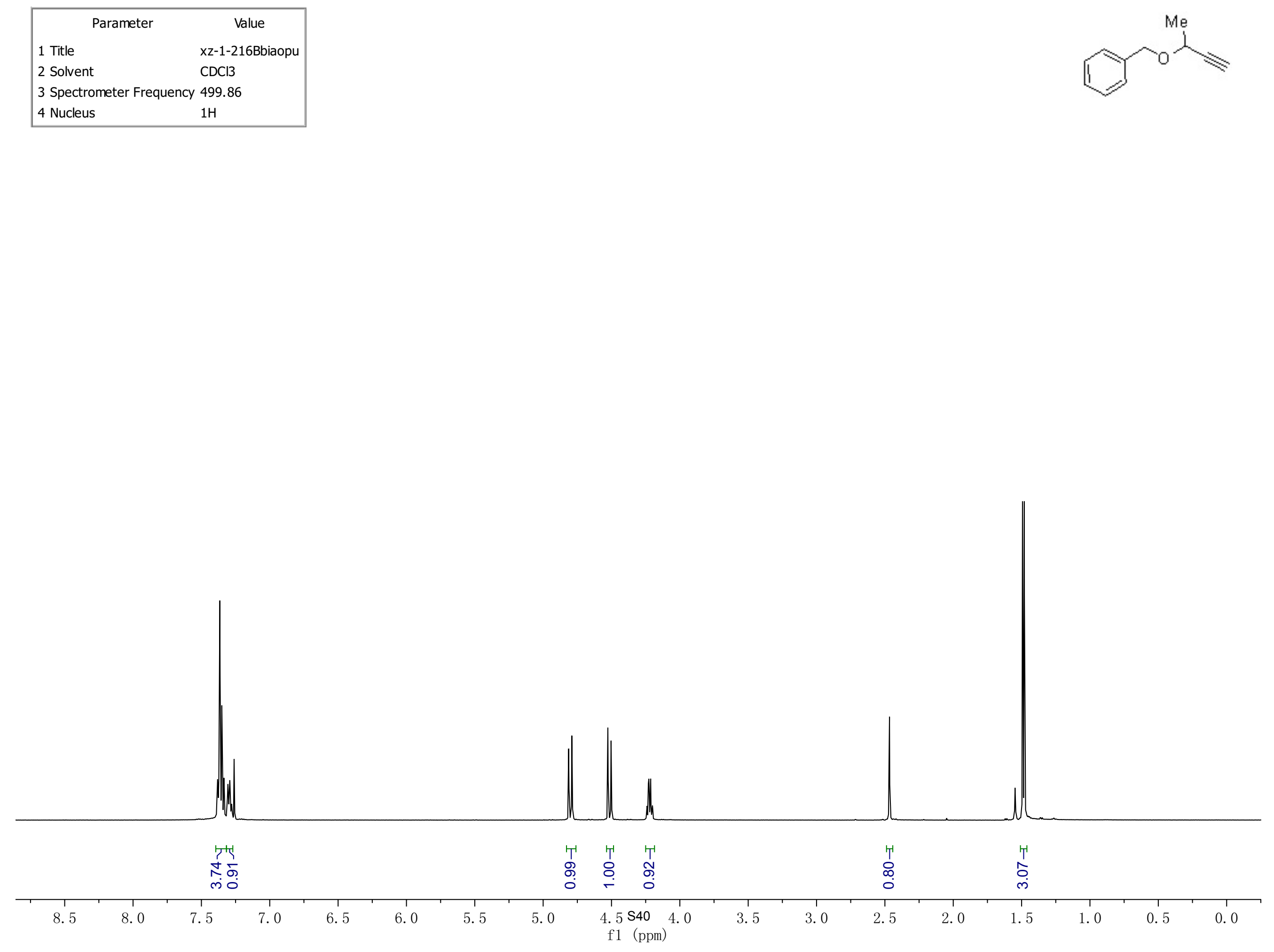


\begin{tabular}{|ll|}
\hline \multicolumn{1}{|c|}{ Parameter } & \multicolumn{1}{c|}{ Value } \\
1 Title & $\mathrm{xz}-1-216 \mathrm{CCbiaopu}$ \\
2 Solvent & $\mathrm{CDCl} 3$ \\
3 Spectrometer Frequency & 125.70 \\
4 Nucleus & $13 \mathrm{C}$ \\
\hline
\end{tabular}
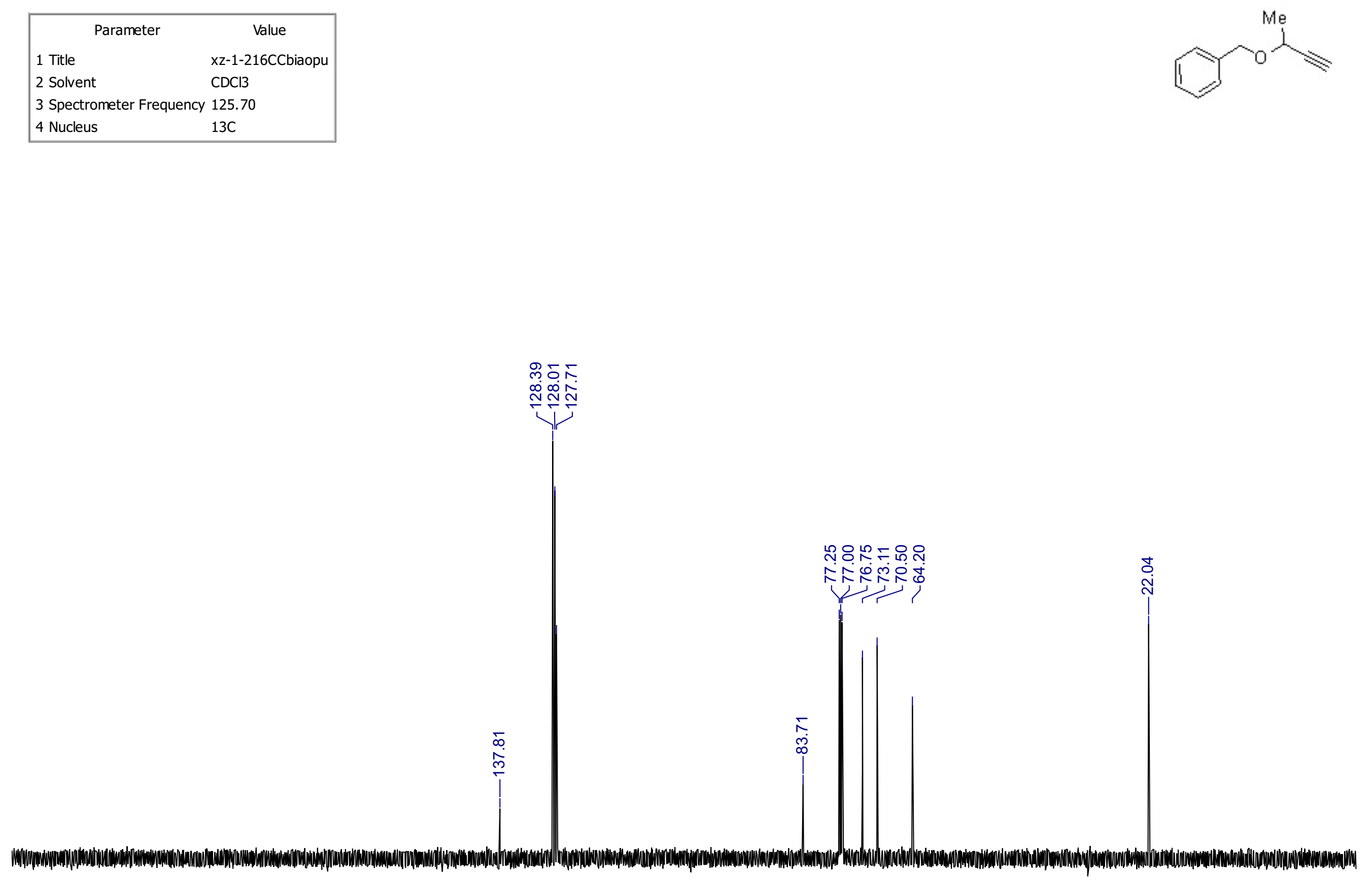

\begin{tabular}{|c|c|c|c|c|c|c|c|c|c|c|c|c|c|c|c|c|c|c|c|c|c|c|}
\hline 1 & 1 & 1 & 1 & 1 & 1 & 1 & 1 & 1 & 1 & 1 & 1911 & 1 & 1 & 1 & 1 & 1 & 1 & $T$ & 1 & 1 & $T$ & 1 \\
\hline 220 & 210 & 200 & 190 & 180 & 170 & 160 & 150 & 140 & 130 & 120 & $\begin{array}{c}110 \text { S41 } 100 \\
\text { f1 (ppm) }\end{array}$ & 90 & 80 & 70 & 60 & 50 & 40 & 30 & 20 & 10 & 0 & -10 \\
\hline
\end{tabular}




\begin{tabular}{|ll|}
\hline \multicolumn{1}{|c|}{ Parameter } & \multicolumn{1}{c|}{ Value } \\
1 Title & $\mathrm{xz}-1-178 \mathrm{BP}$ (1) \\
2 Solvent & $\mathrm{cdcl3}$ \\
3 Spectrometer Frequency & 399.78 \\
4 Nucleus & $1 \mathrm{H}$ \\
\hline
\end{tabular}

Spectrometer Frequency 3

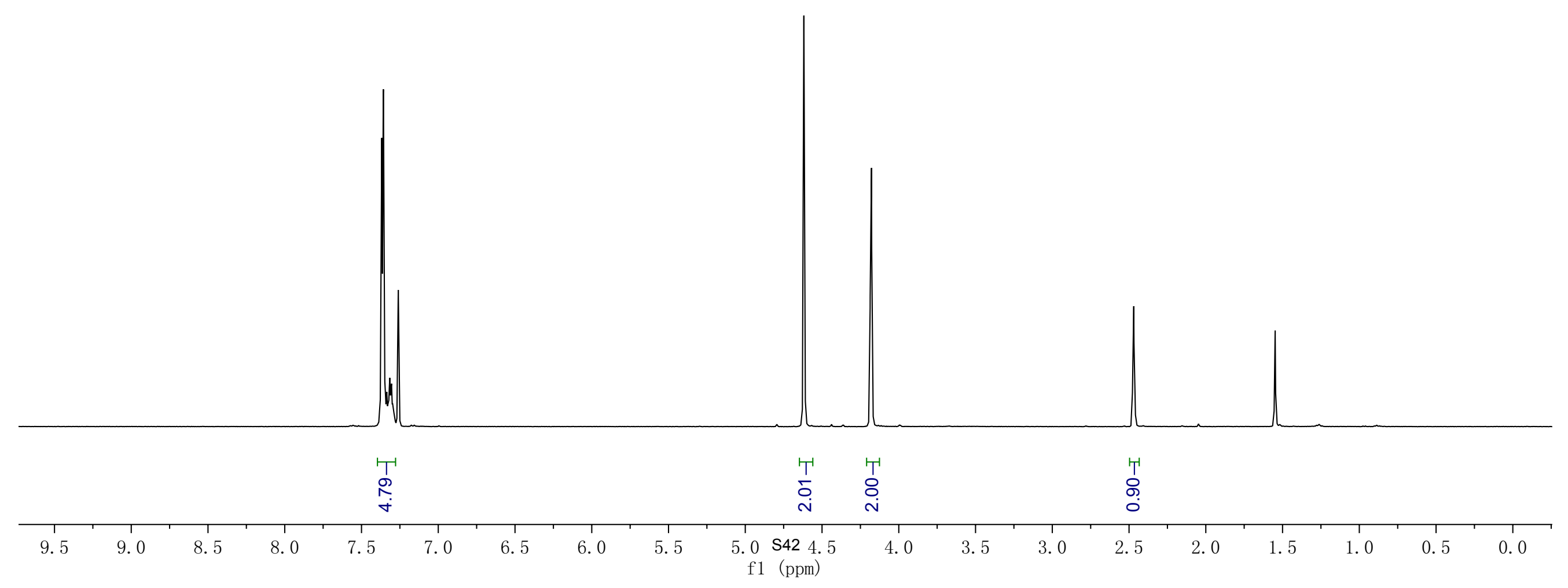




\begin{tabular}{|ll|}
\hline \multicolumn{1}{|c|}{ Parameter } & \multicolumn{1}{c|}{ Value } \\
1 Title & $\mathrm{xz}-1-178 \mathrm{BCbiaopu} 500$ \\
2 Solvent & $\mathrm{CDCl} 3$ \\
3 Spectrometer Frequency & 125.70 \\
4 Nucleus & $13 \mathrm{C}$ \\
\hline
\end{tabular}

trometer Frequency 125

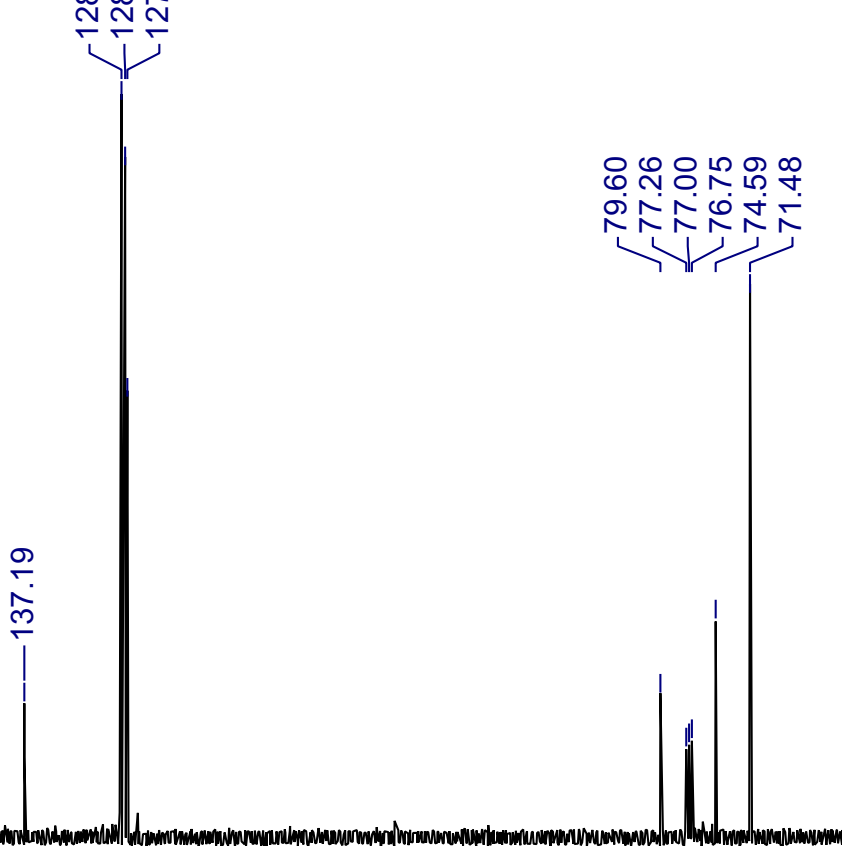

\begin{tabular}{|c|c|c|c|c|c|c|c|c|c|c|c|c|c|c|c|c|c|c|c|c|c|}
\hline 220 & 210 & 200 & 190 & 180 & 170 & 160 & 150 & 140 & 130 & 120 & $\begin{array}{l}110 \mathrm{~S} 43100 \\
\mathrm{f} 1(\mathrm{ppm})\end{array}$ & 90 & 80 & 70 & 60 & 50 & 40 & 30 & 20 & 10 & -10 \\
\hline
\end{tabular}




\begin{tabular}{|ll|}
\hline \multicolumn{1}{|c|}{ Parameter } & \multicolumn{1}{c|}{ Value } \\
1 Title & $\mathrm{xz}-1-213 \mathrm{AH} 500$ \\
2 Solvent & $\mathrm{CDCl} 3$ \\
3 Spectrometer Frequency & 499.86 \\
4 Nucleus & $1 \mathrm{H}$ \\
\hline
\end{tabular}
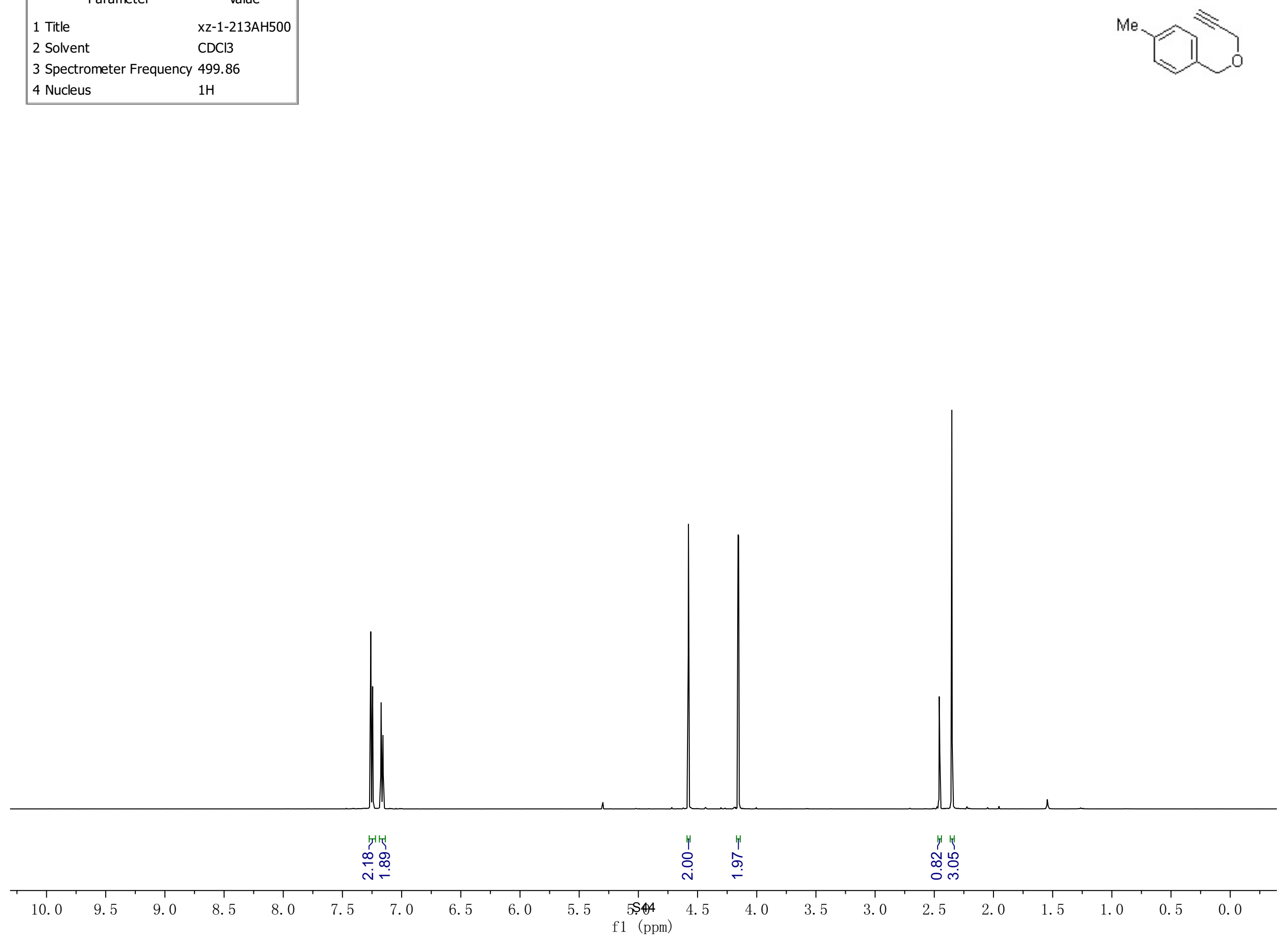


\begin{tabular}{|ll|}
\hline \multicolumn{1}{|c|}{ Parameter } & \multicolumn{1}{c|}{ Value } \\
1 Title & $\mathrm{xz}-1-213 \mathrm{AC} 500$ \\
2 Solvent & $\mathrm{CDCl3}$ \\
3 Spectrometer Frequency & 125.70 \\
4 Nucleus & $13 \mathrm{C}$ \\
\hline
\end{tabular}

츙ํำำ

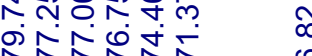

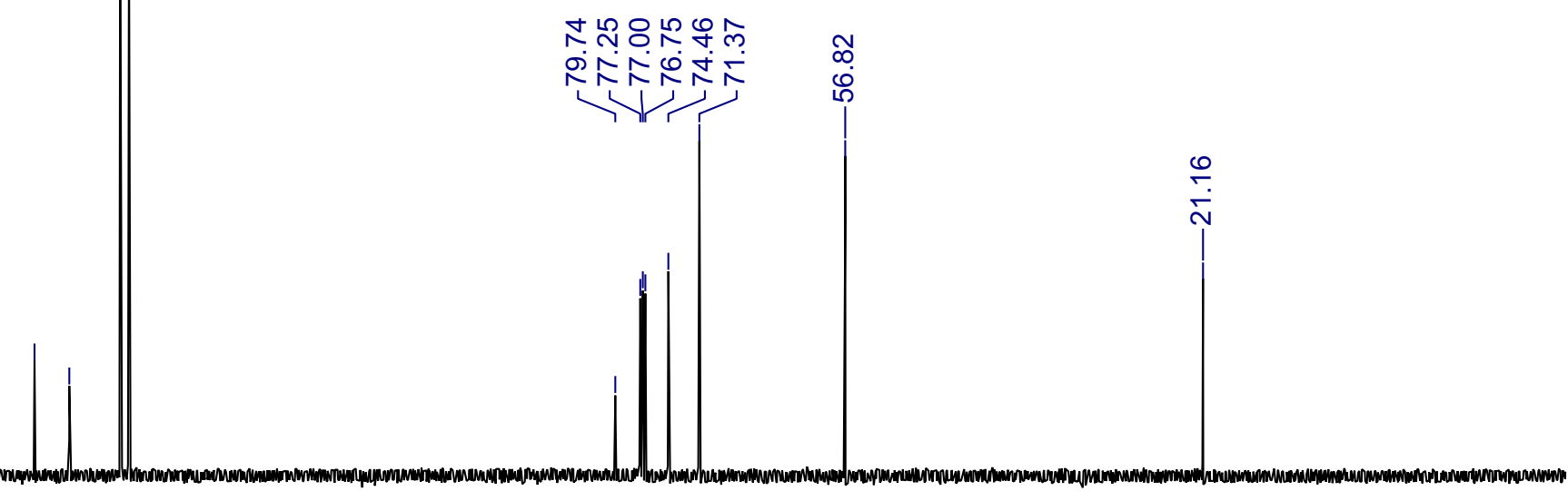

\begin{tabular}{|c|c|c|c|c|c|c|c|c|c|c|c|c|c|c|c|c|c|c|c|c|c|c|}
\hline 220 & 210 & 200 & $\begin{array}{l}1 \\
190\end{array}$ & $\begin{array}{l}1 \\
180\end{array}$ & $\begin{array}{l}1 \\
170\end{array}$ & 160 & 150 & 140 & $\begin{array}{c}1 \\
130\end{array}$ & $\begin{array}{l}1 \\
120\end{array}$ & $\begin{array}{l}110 \text { S45 } 100 \\
\text { f1 (ppm) }\end{array}$ & 90 & $\begin{array}{l}1 \\
80\end{array}$ & 70 & 60 & $\begin{array}{l}1 \\
50\end{array}$ & 40 & 30 & $\begin{array}{l}1 \\
20\end{array}$ & $\begin{array}{l}1 \\
10\end{array}$ & 0 & -10 \\
\hline
\end{tabular}




\begin{tabular}{|ll|}
\hline \multicolumn{1}{|c|}{ Parameter } & \multicolumn{1}{c|}{ Value } \\
1 Title & chy-3-74-1-SM-1H \\
2 Solvent & $\mathrm{CDCl} 3$ \\
3 Spectrometer Frequency & 499.86 \\
4 Nucleus & $1 \mathrm{H}$ \\
\hline
\end{tabular}
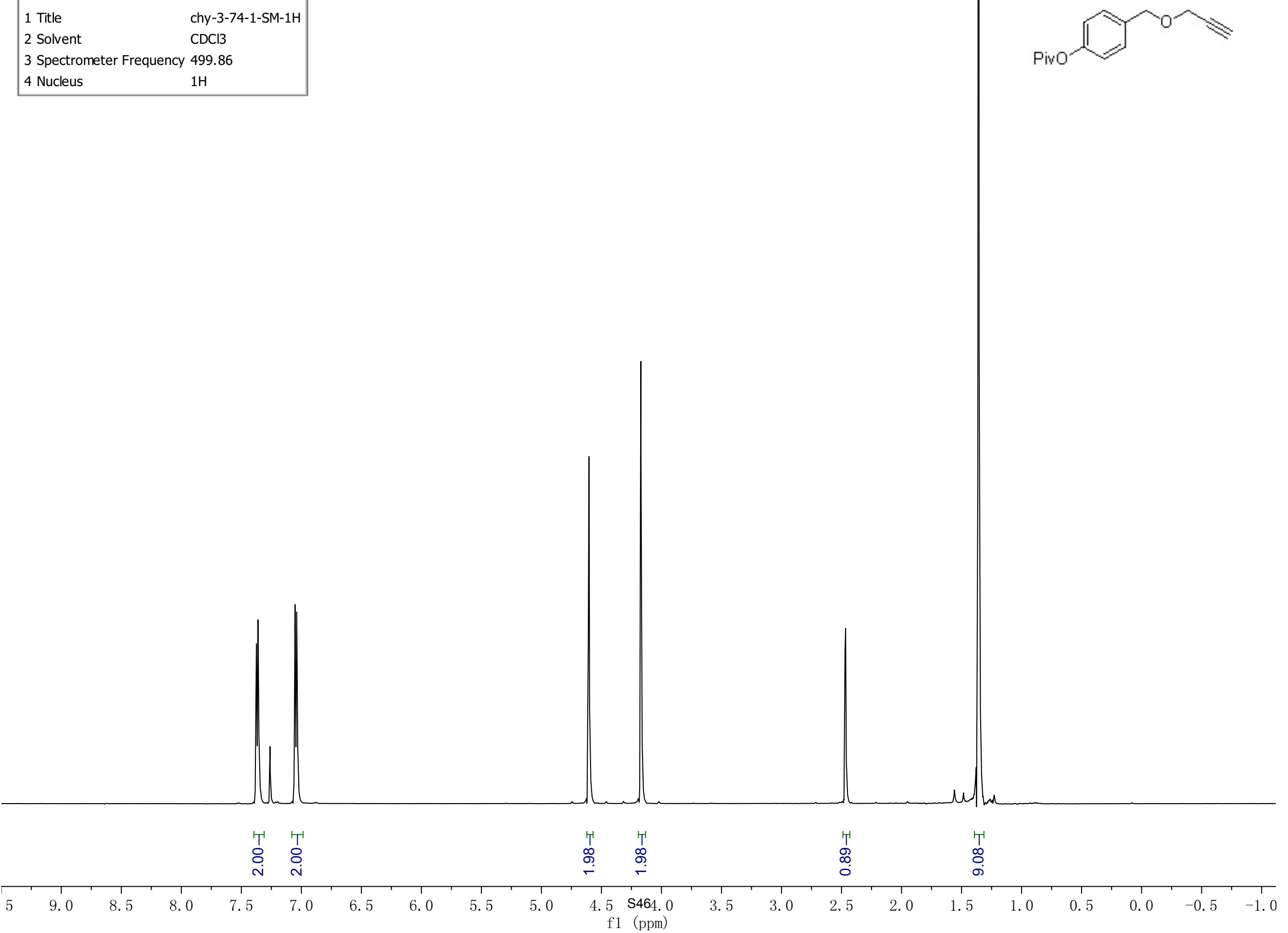


\begin{tabular}{|ll|}
\hline \multicolumn{1}{|c|}{ Parameter } & \multicolumn{1}{c|}{ Value } \\
1 Title & chy-3-74-1-SM-13C \\
2 Solvent & CDCl3 \\
3 Spectrometer Frequency & 125.70 \\
4 Nucleus & $13 \mathrm{C}$ \\
\hline
\end{tabular}
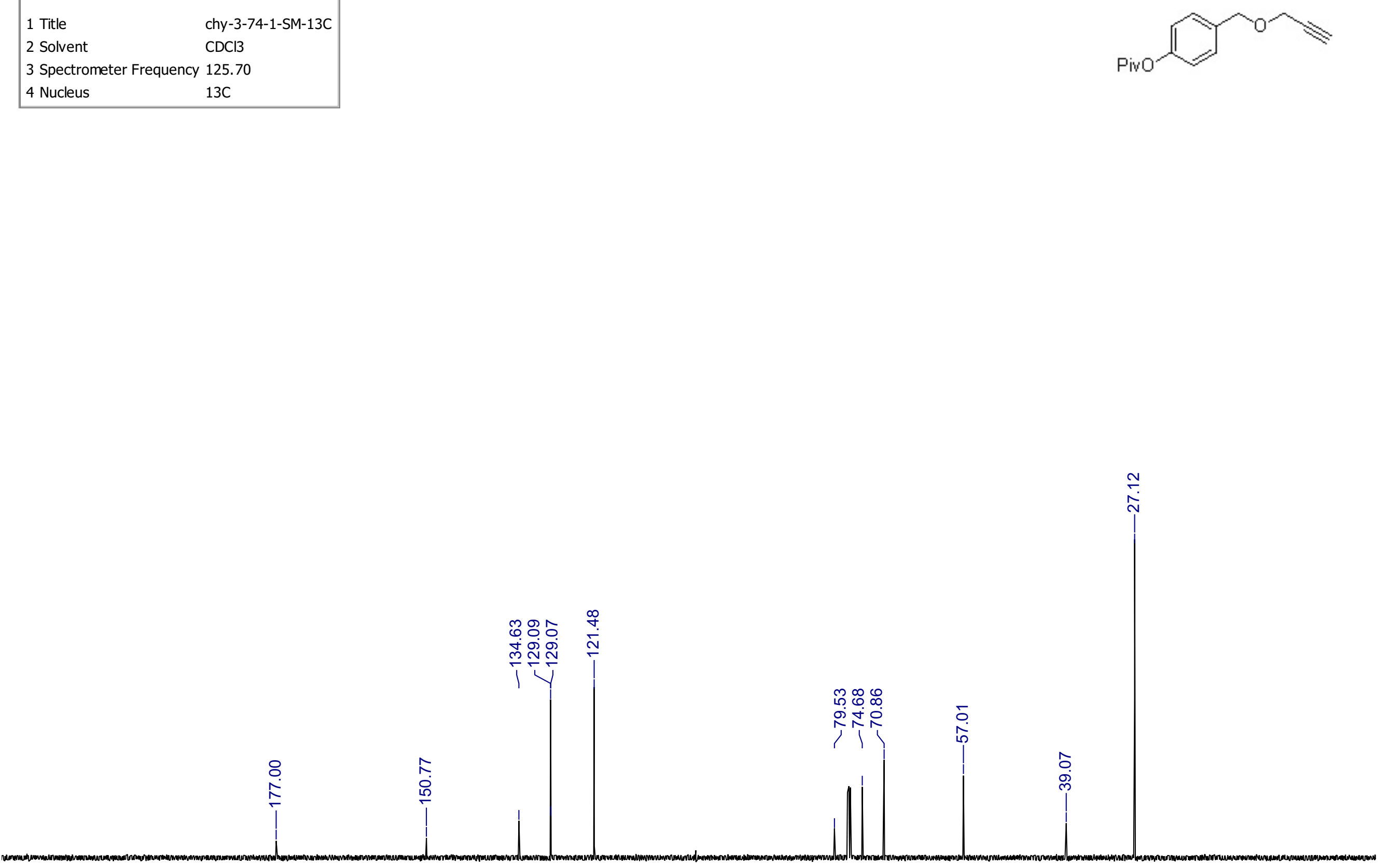

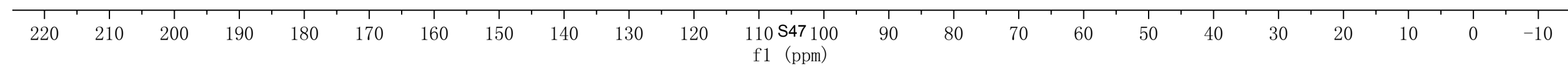




\begin{tabular}{|ll|}
\hline \multicolumn{1}{|c|}{ Parameter } & \multicolumn{1}{c|}{ Value } \\
1 Title & chy-3-75-1-SM-1H \\
2 Solvent & $\mathrm{CDCl} 3$ \\
3 Spectrometer Frequency & 499.86 \\
4 Nucleus & $1 \mathrm{H}$ \\
\hline
\end{tabular}
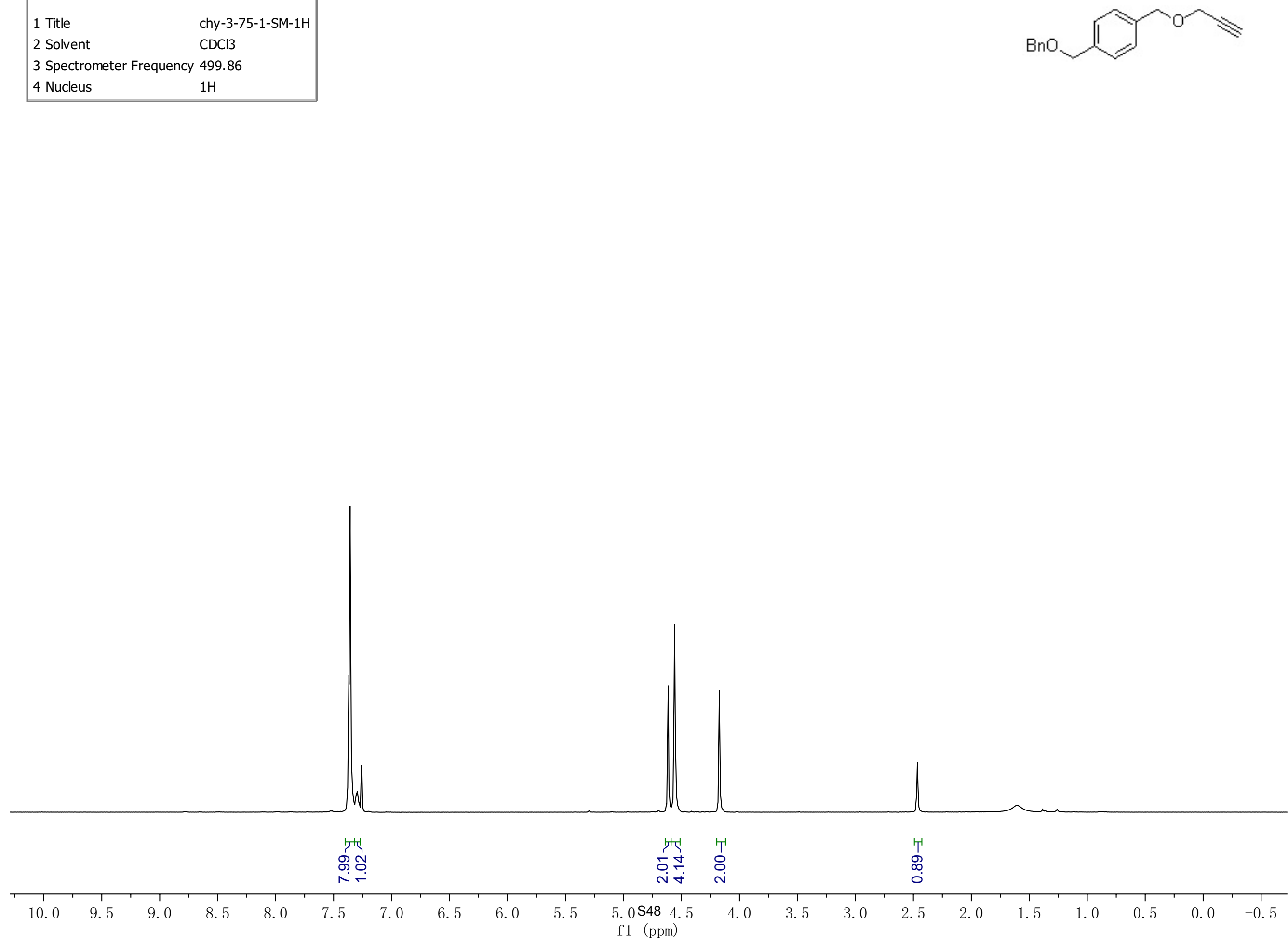


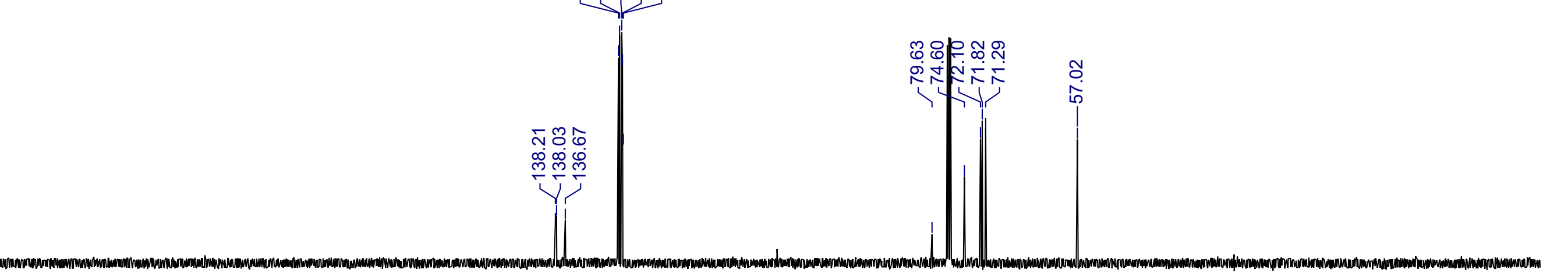

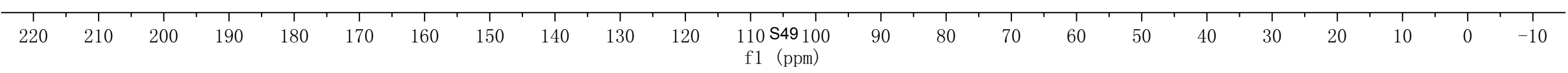




\begin{tabular}{|ll|}
\hline \multicolumn{1}{|c|}{ Parameter } & \multicolumn{1}{c|}{ Value } \\
1 Title & chy-3-106-1-SM-1H \\
2 Solvent & $\mathrm{CDCl} 3$ \\
3 Spectrometer Frequency & 499.86 \\
4 Nucleus & $1 \mathrm{H}$ \\
\hline
\end{tabular}
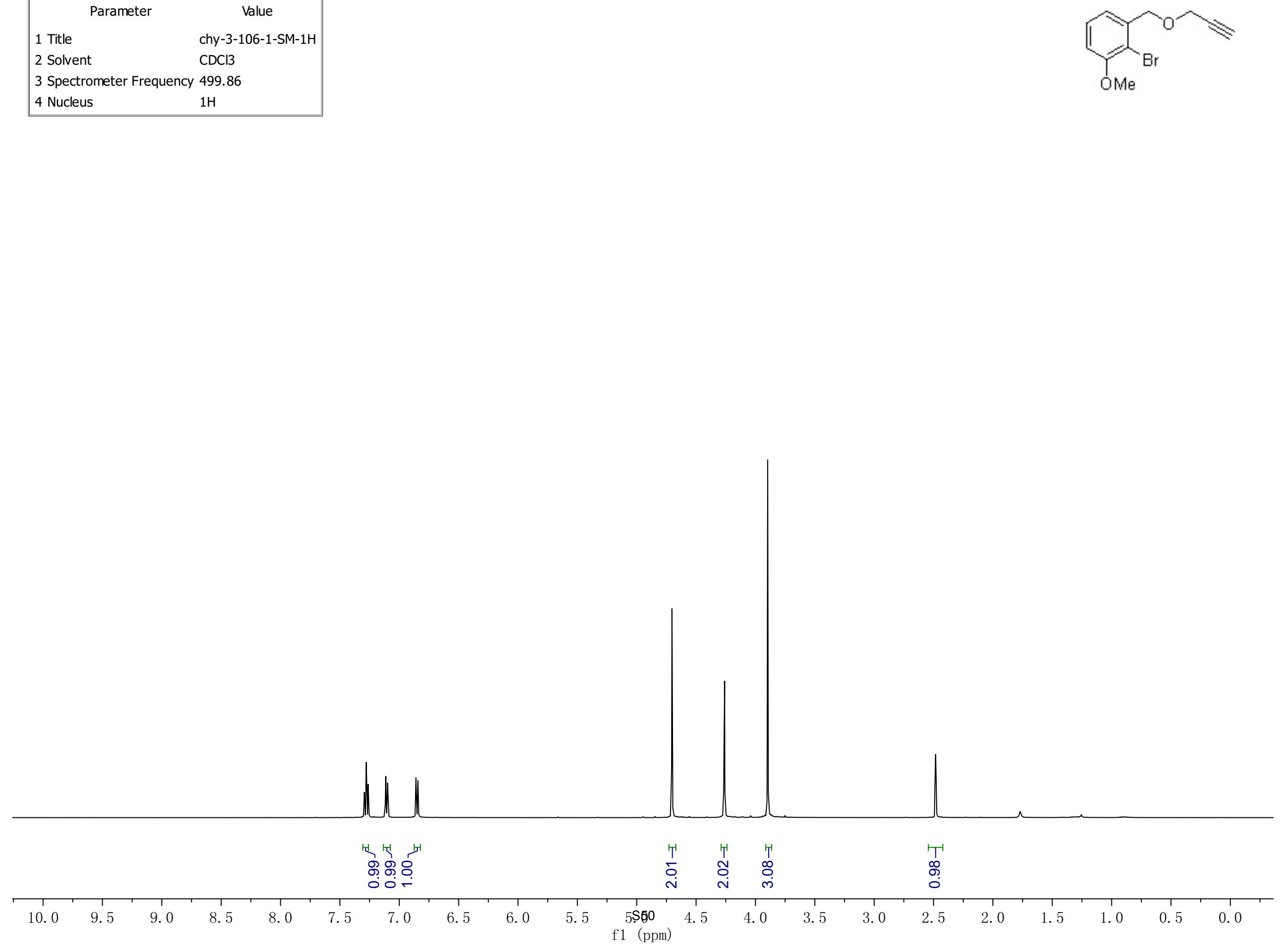
3 Spectrometer Frequency 125.70
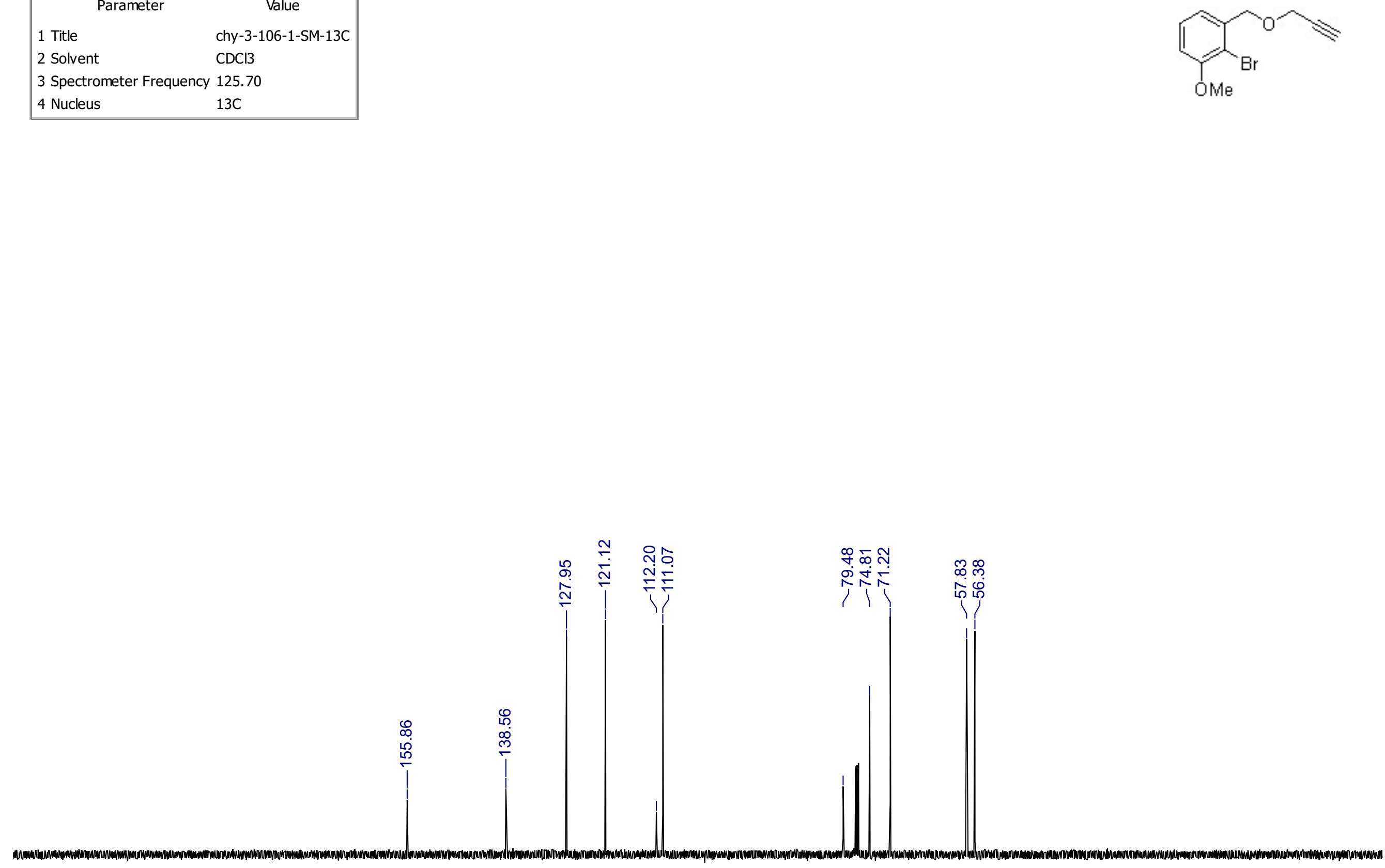

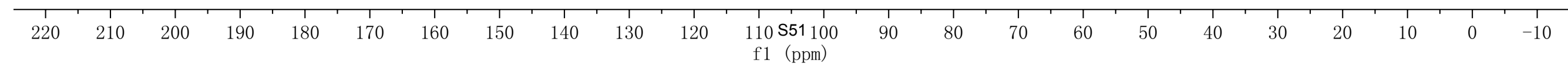




\begin{tabular}{|c|c|}
\hline Parameter & Value \\
\hline 1 Title & $x z-1-210-S M-1$ \\
\hline 2 Solvent & $\mathrm{CDCl} 3$ \\
\hline 3 Spectrometer Frequen & 499.86 \\
\hline 4 Nucleus & $1 \mathrm{H}$ \\
\hline
\end{tabular}
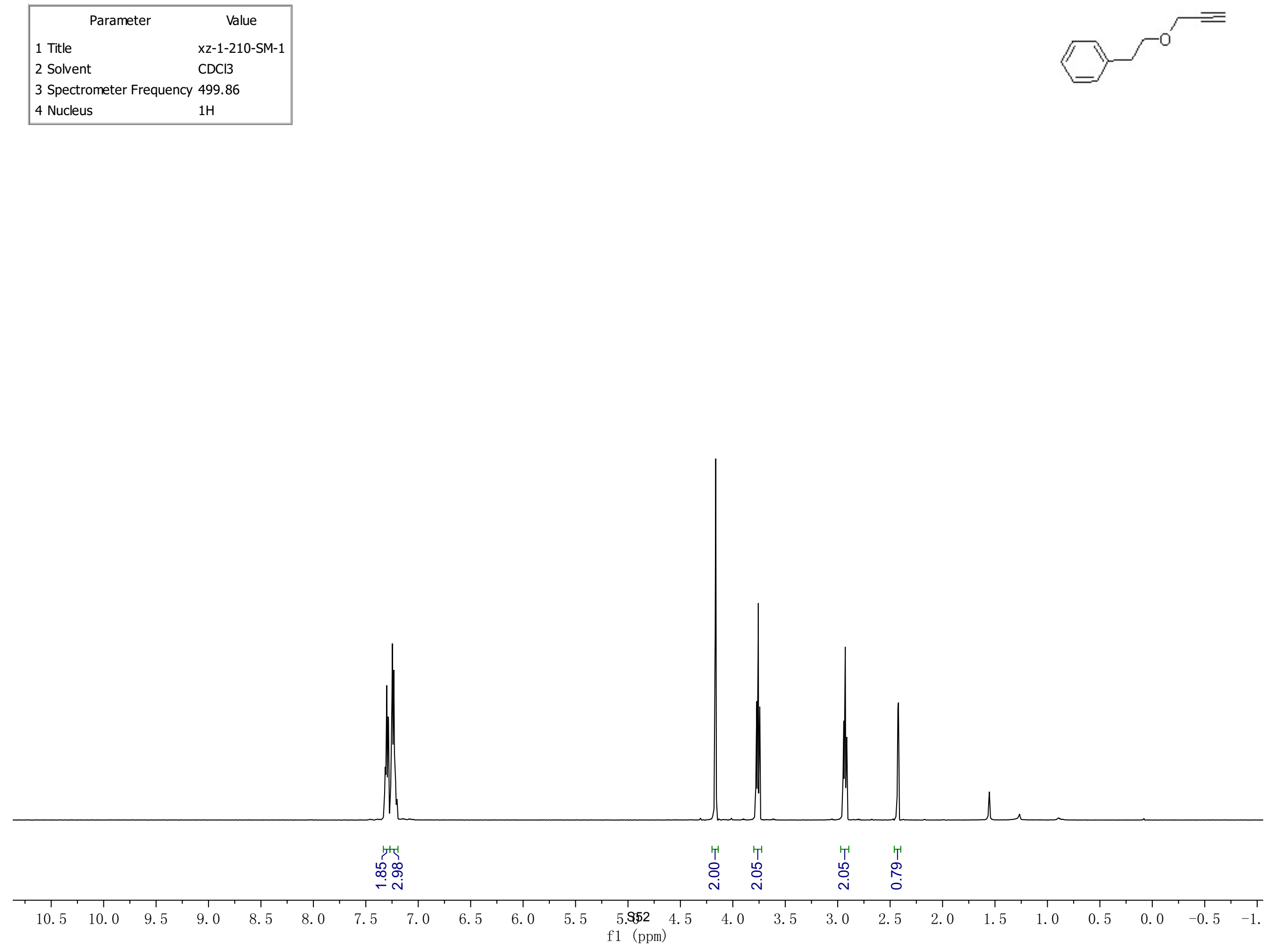


\begin{tabular}{|ll|}
\hline \multicolumn{1}{|c|}{ Parameter } & \multicolumn{1}{c|}{ Value } \\
1 Title & $\mathrm{xz}-1-\mathrm{SM}$-210Cbiaopu \\
2 Solvent & $\mathrm{CDCl} 3$ \\
3 Spectrometer Frequency & 125.70 \\
4 Nucleus & $13 \mathrm{C}$ \\
\hline
\end{tabular}
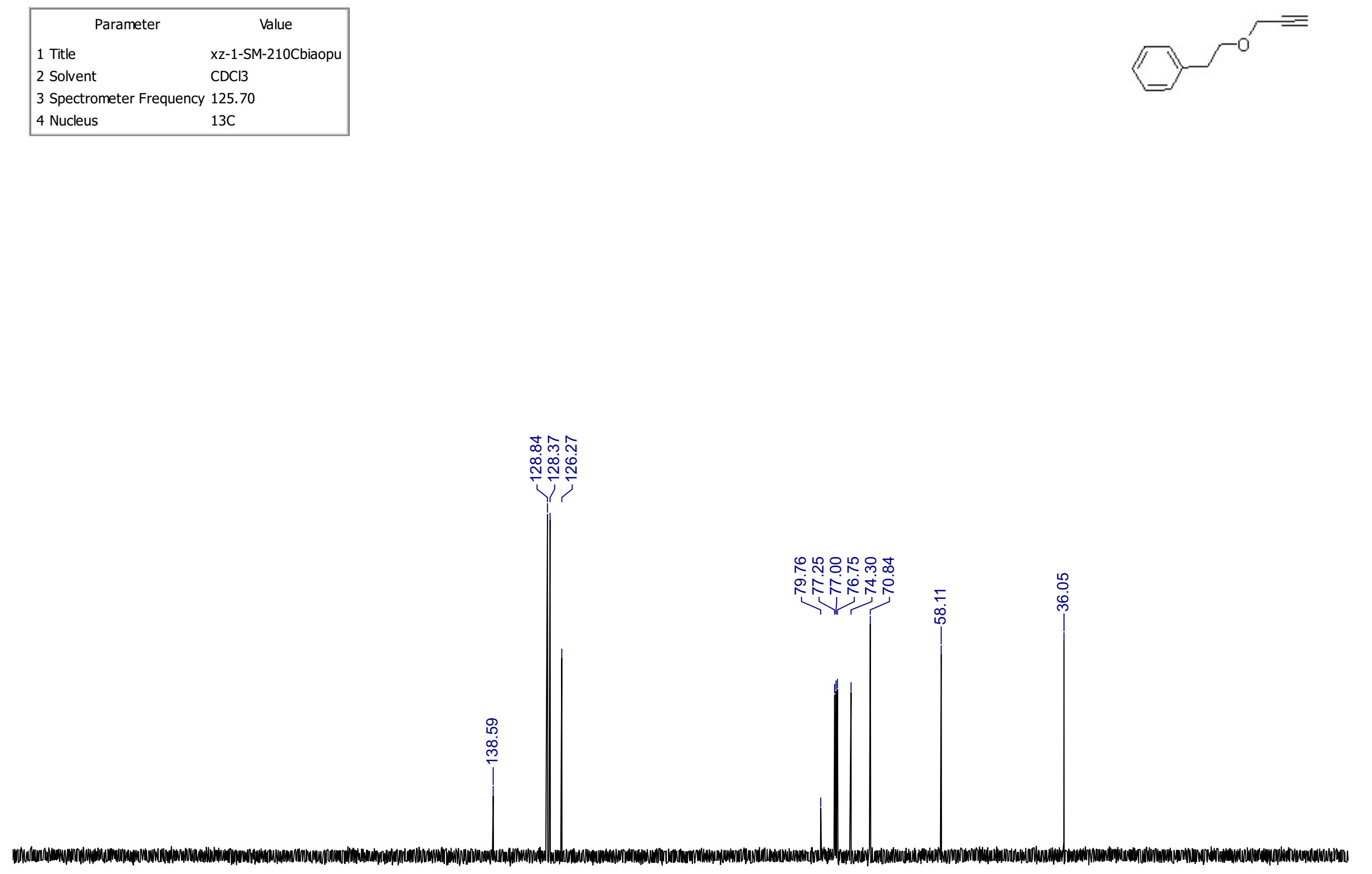

\begin{tabular}{|c|c|c|c|c|c|c|c|c|c|c|c|c|c|c|c|c|c|c|c|c|c|c|}
\hline 1 & 1 & 1 & 1 & 1 & 1 & 1 & 1 & 1 & 1 & 1 & 1 & 1 & 1 & 1 & 1 & 1 & 1 & 1 & 1 & 1 & 1 & 1 \\
\hline 220 & 210 & 200 & 190 & 180 & 170 & 160 & 150 & 140 & 130 & 120 & $\begin{array}{c}110 \text { S53 } 100 \\
\text { f1 (ppm) }\end{array}$ & 90 & 80 & 70 & 60 & 50 & 40 & 30 & 20 & 10 & 0 & -10 \\
\hline
\end{tabular}




\begin{tabular}{|ll|}
\hline \multicolumn{1}{|c|}{ Parameter } & \multicolumn{1}{c|}{ Value } \\
1 Title & $\mathrm{xz}-1-256 \mathrm{H}$ \\
2 Solvent & $\mathrm{CDCl} 3$ \\
3 Spectrometer Frequency & 499.86 \\
4 Nucleus & $1 \mathrm{H}$ \\
\hline
\end{tabular}<smiles>C#CCOCCc1ccc(C)cc1</smiles>

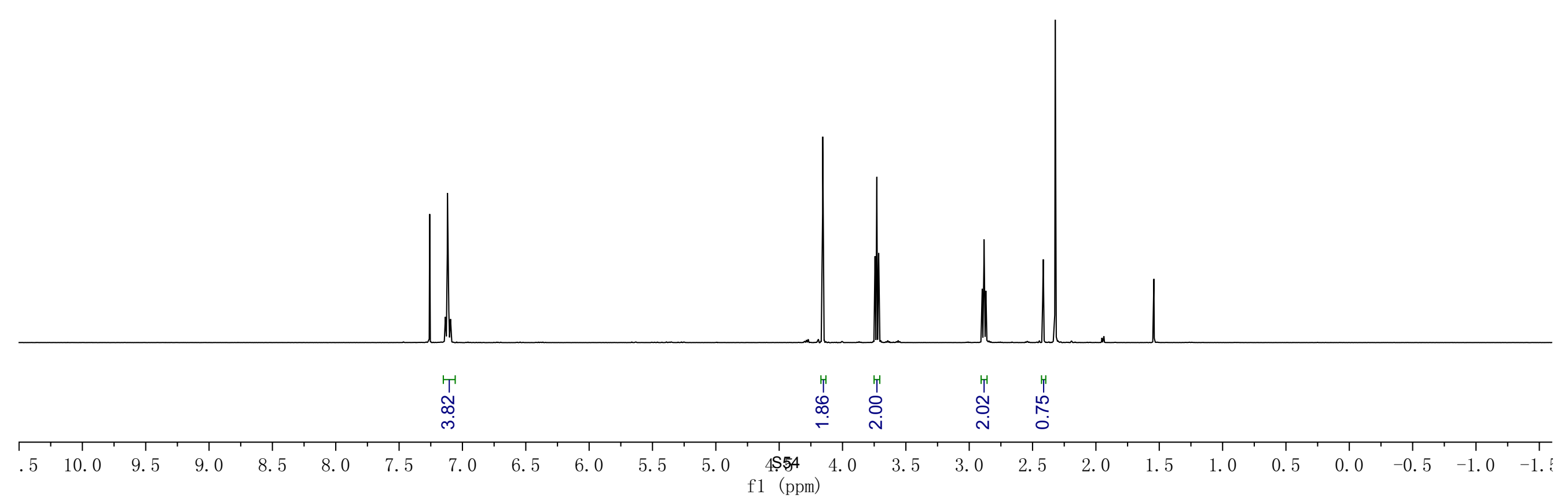




\begin{tabular}{|ll|}
\hline \multicolumn{1}{|c|}{ Parameter } & \multicolumn{1}{c|}{ Value } \\
1 Title & $\mathrm{xz}-1-256 \mathrm{C}$ \\
2 Solvent & $\mathrm{CDCl} 3$ \\
3 Spectrometer Frequency & 125.70 \\
4 Nucleus & $13 \mathrm{C}$ \\
\hline
\end{tabular}
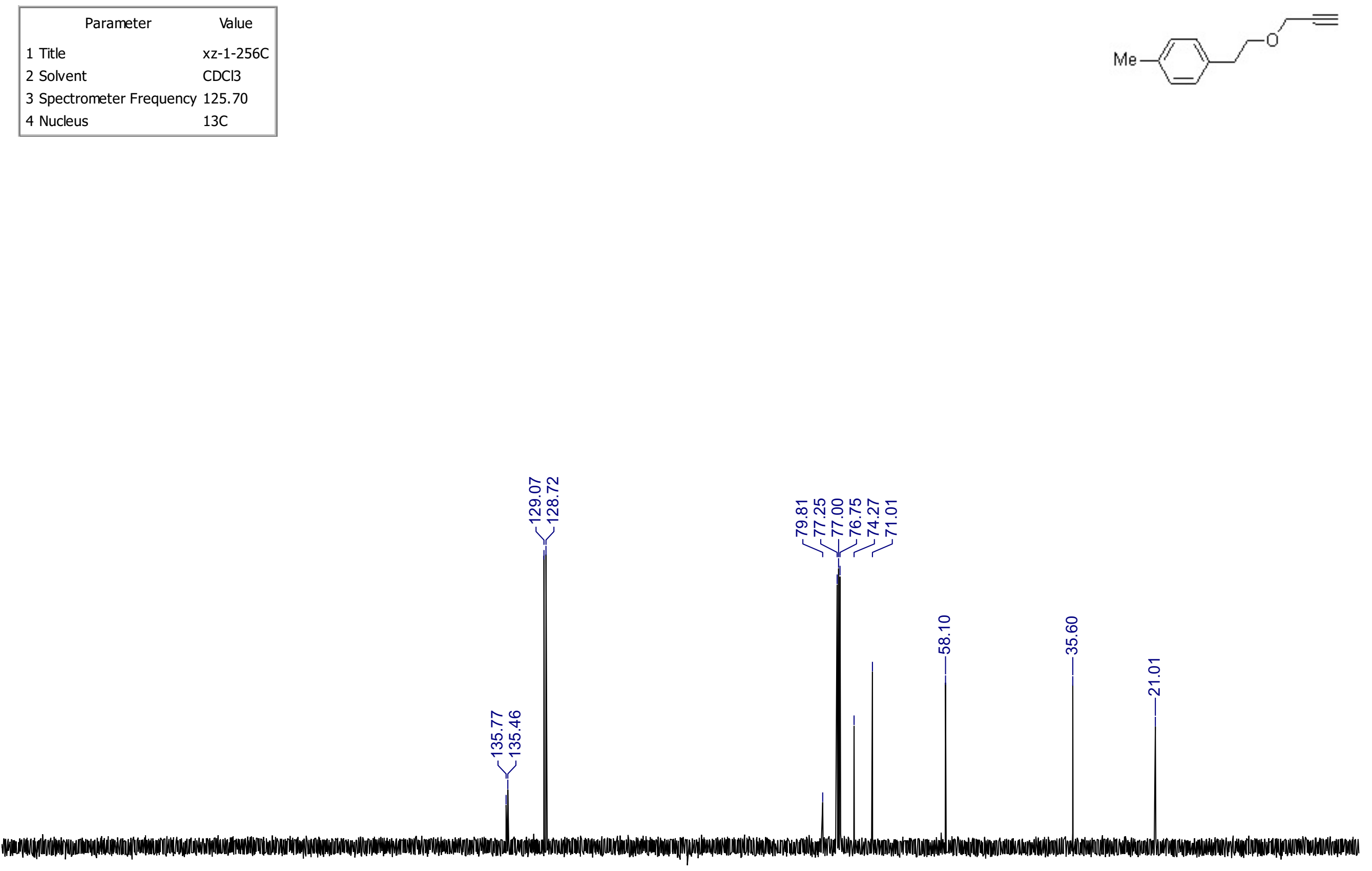

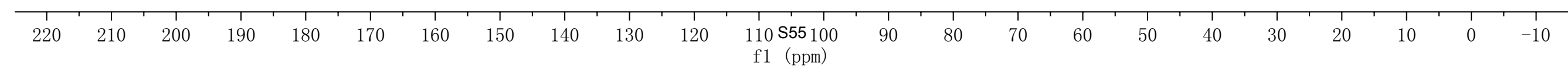




\begin{tabular}{|ll|}
\hline \multicolumn{1}{|c|}{ Parameter } & \multicolumn{1}{c|}{ Value } \\
1 Title & $\mathrm{xz}-1-287 \mathrm{BHbiaopu}$ \\
2 Solvent & $\mathrm{CDCl3}$ \\
3 Spectrometer Frequency & 499.86 \\
4 Nucleus & $1 \mathrm{H}$ \\
\hline
\end{tabular}
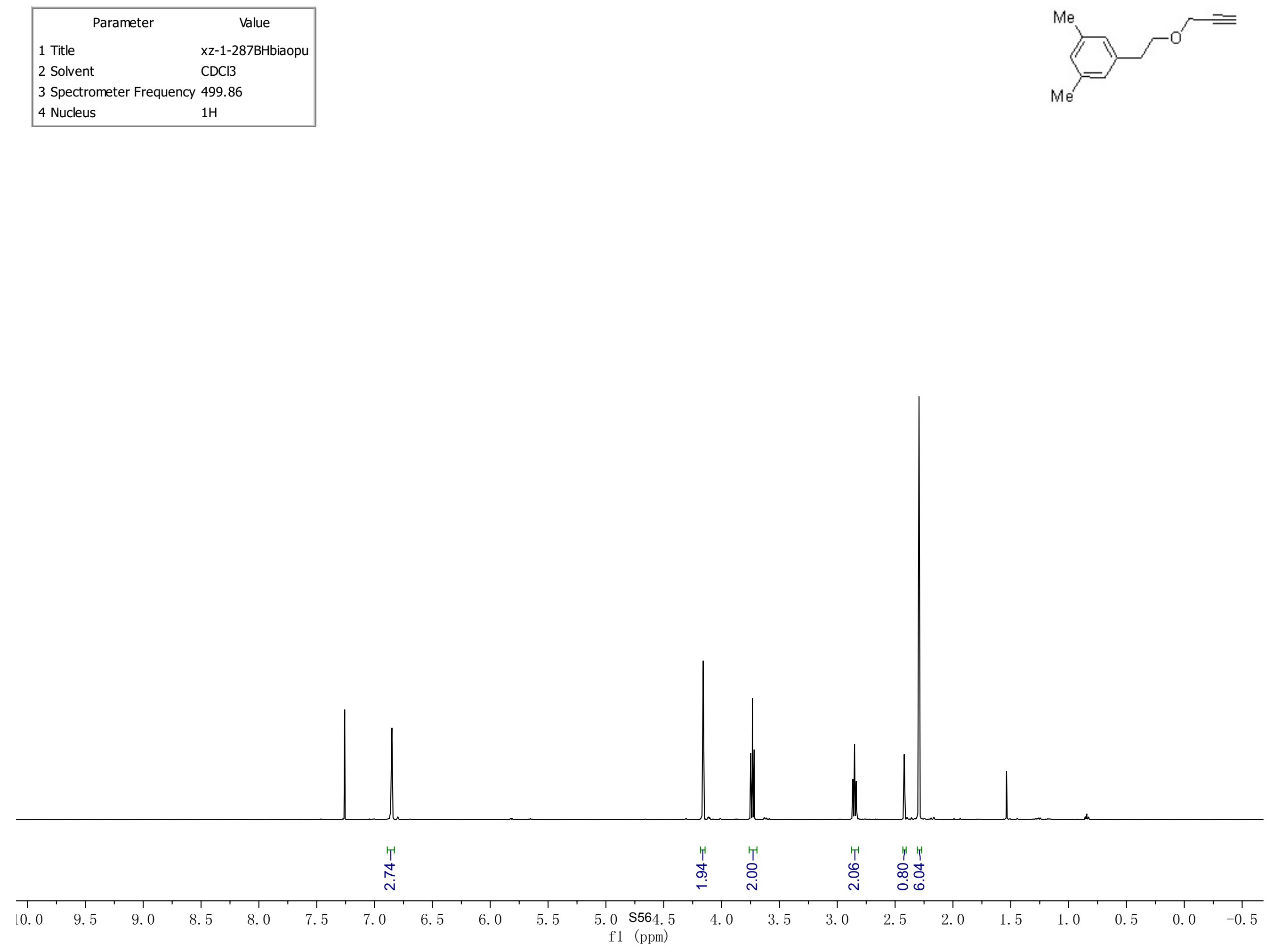


\begin{tabular}{|ll|}
\hline \multicolumn{1}{|c|}{ Parameter } & \multicolumn{1}{c|}{ Value } \\
1 Title & $\mathrm{xz}-1-287 \mathrm{BCbiaopu}$ \\
2 Solvent & $\mathrm{CDCl} 3$ \\
3 Spectrometer Frequency & 125.70 \\
4 Nucleus & $13 \mathrm{C}$ \\
\hline
\end{tabular}
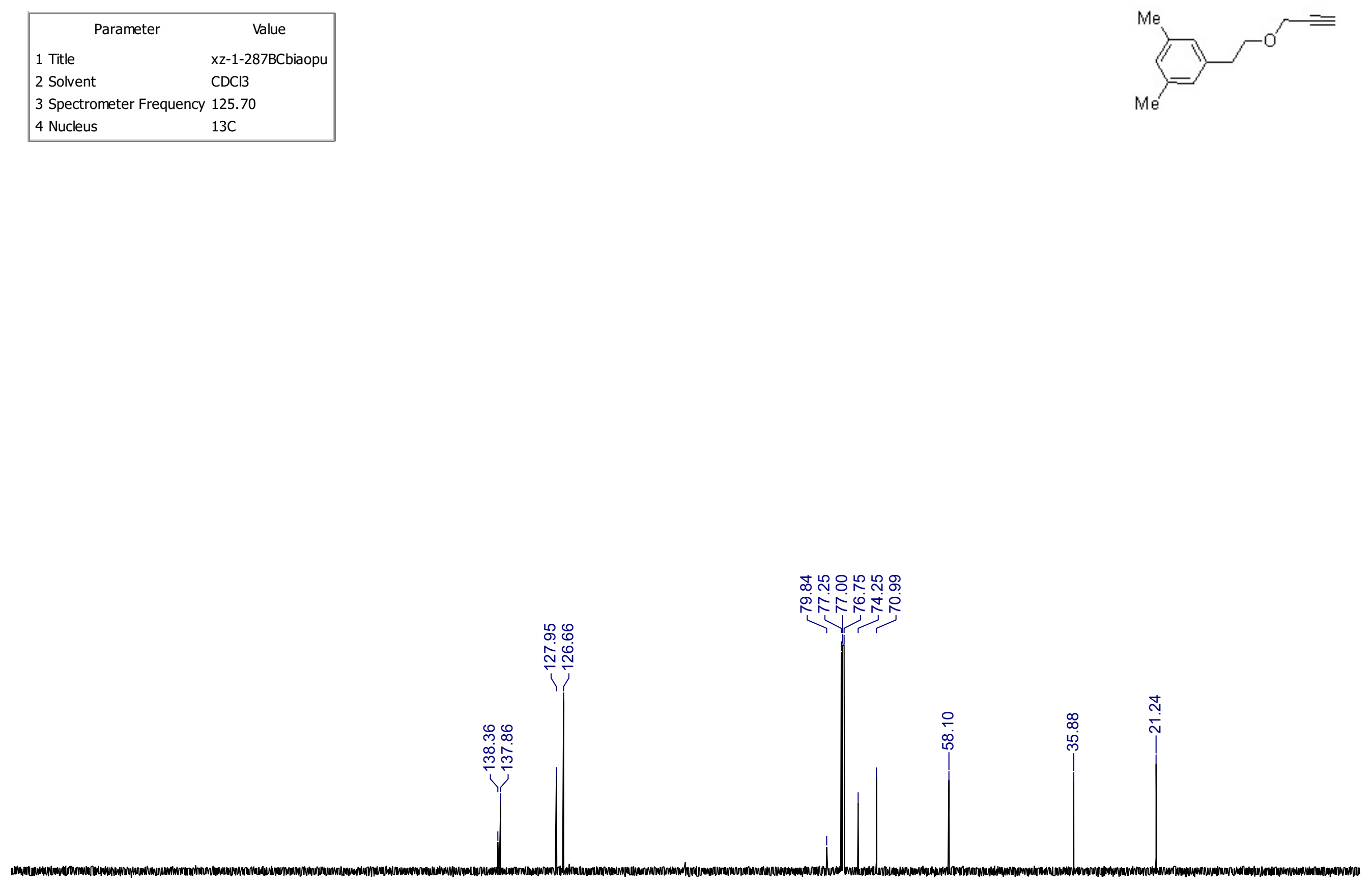

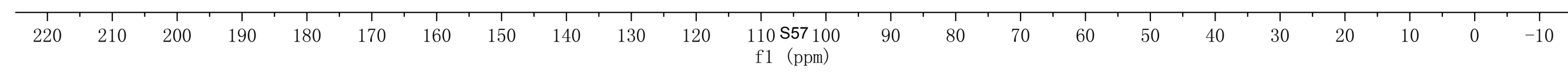




\begin{tabular}{|ll|}
\hline \multicolumn{1}{|c|}{ Parameter } & \multicolumn{1}{c|}{ Value } \\
1 Title & $\mathrm{xz}-1-235 \mathrm{CH}$ \\
2 Solvent & $\mathrm{CDCl} 3$ \\
3 Spectrometer Frequency & 499.86 \\
4 Nucleus & $1 \mathrm{H}$ \\
\hline
\end{tabular}
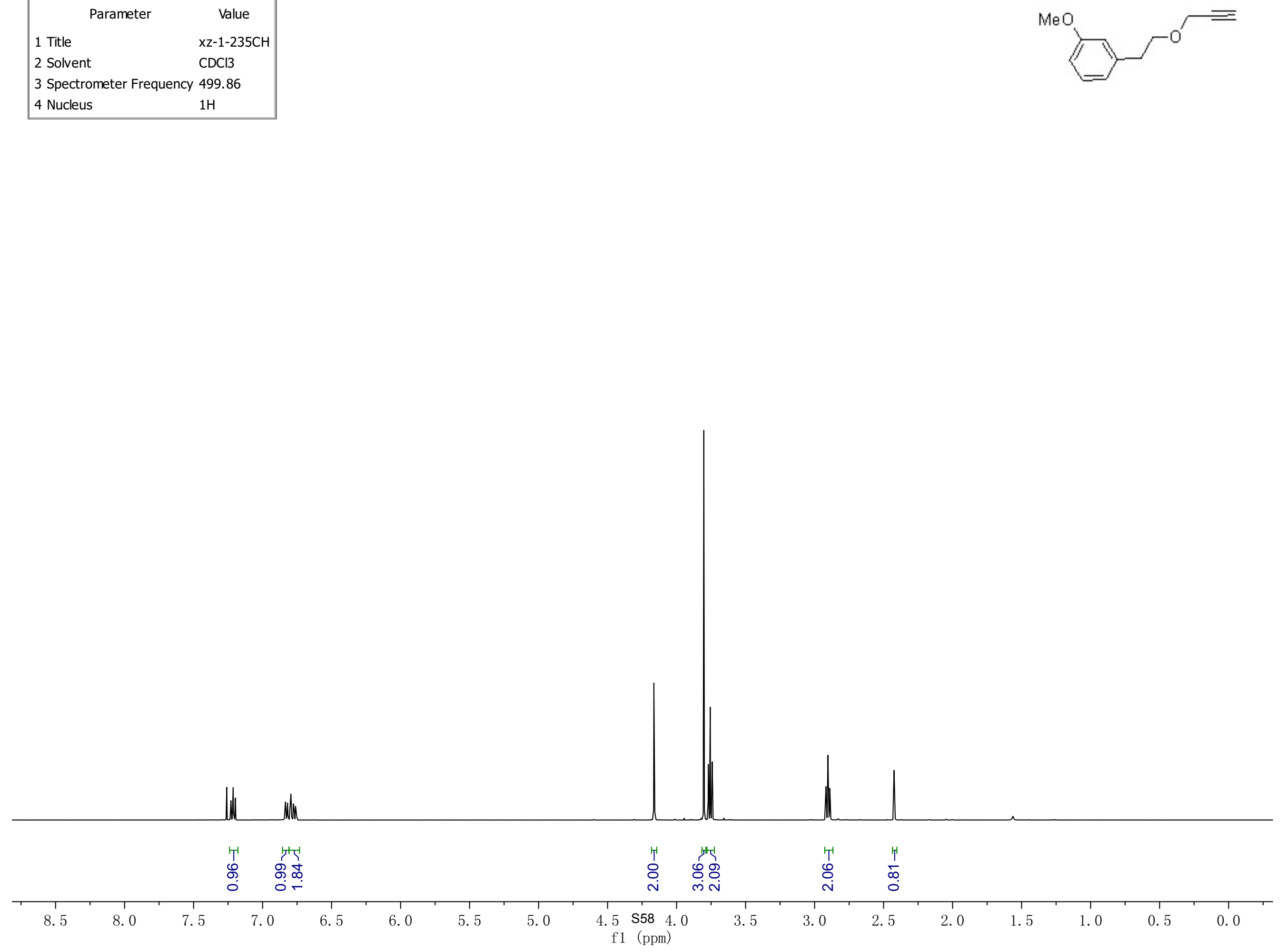


\begin{tabular}{|ll|}
\hline \multicolumn{1}{|c|}{ Parameter } & \multicolumn{1}{c|}{ Value } \\
1 Title & $\mathrm{xz}-235 \mathrm{~B}-\mathrm{C}$ \\
2 Solvent & $\mathrm{CDCl} 3$ \\
3 Spectrometer Frequency & 125.70 \\
4 Nucleus & $13 \mathrm{C}$ \\
\hline
\end{tabular}
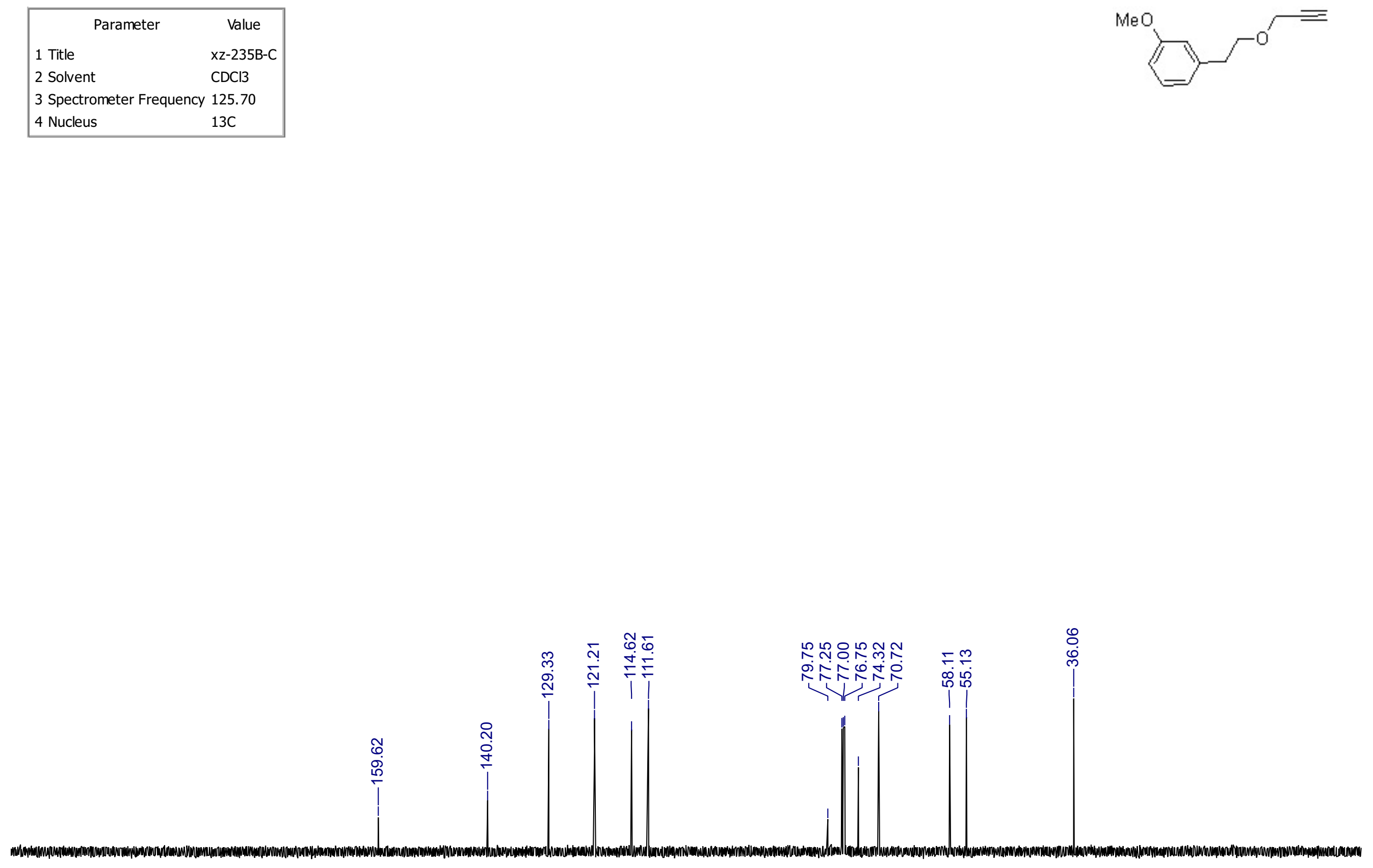

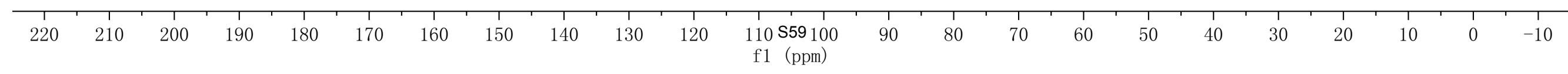




\begin{tabular}{|c|c|}
\hline Parameter & Value \\
\hline 1 Title & $x z-1-S M-239 H$ \\
\hline 2 Solvent & $\mathrm{CDCl} 3$ \\
\hline 3 Spectrometer Frequen & y 499.86 \\
\hline 4 Nucleus & $1 \mathrm{H}$ \\
\hline
\end{tabular}
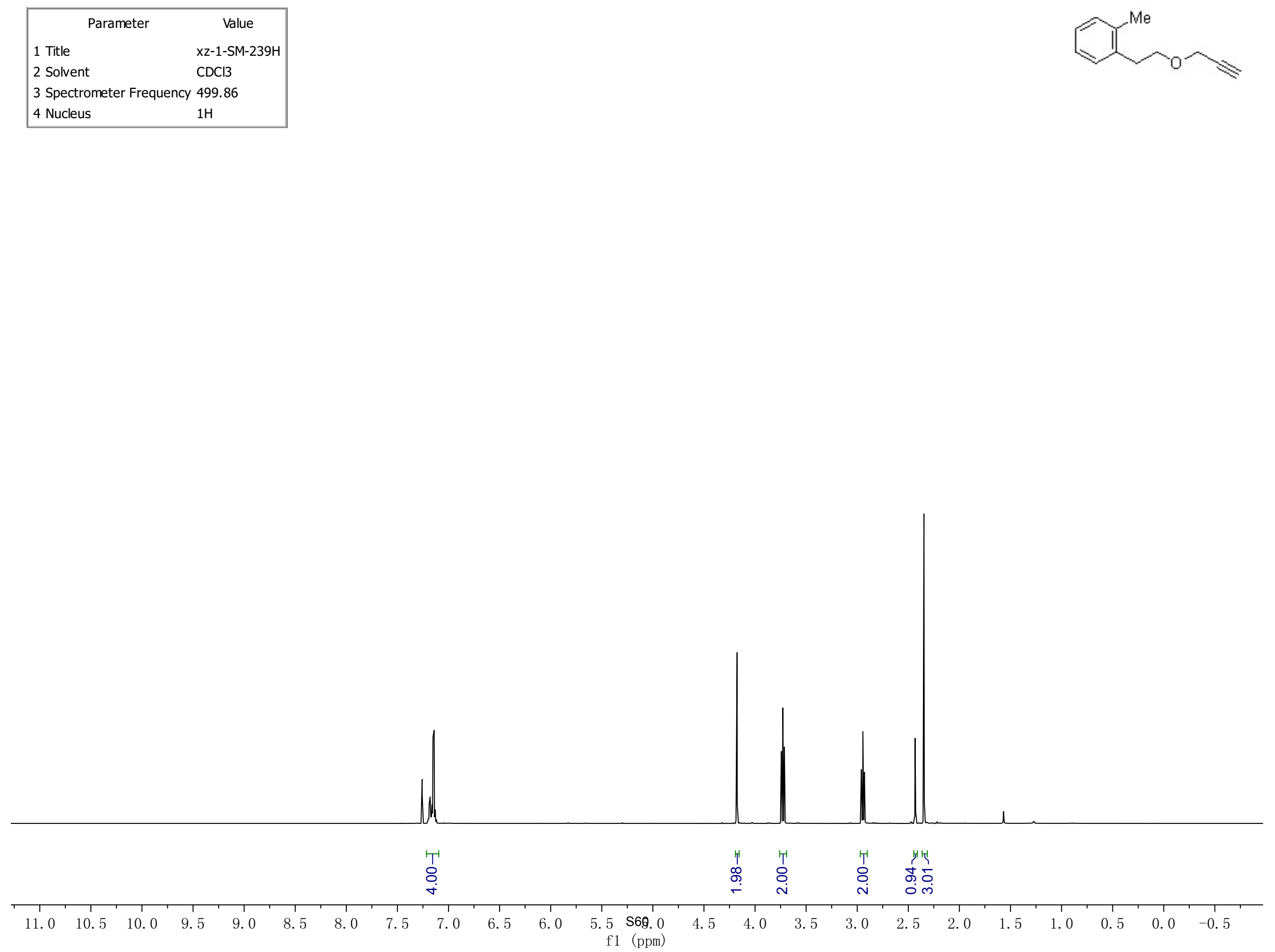


\begin{tabular}{|ll|}
\hline \multicolumn{1}{|c|}{ Parameter } & \multicolumn{1}{c|}{ Value } \\
1 Title & $\mathrm{xz}-1-\mathrm{SM}-239 \mathrm{C}$ \\
2 Solvent & $\mathrm{CDCl3}$ \\
3 Spectrometer Frequency & 125.70 \\
4 Nucleus & $13 \mathrm{C}$ \\
\hline
\end{tabular}

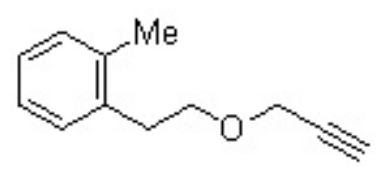

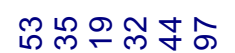

फ़्户̄

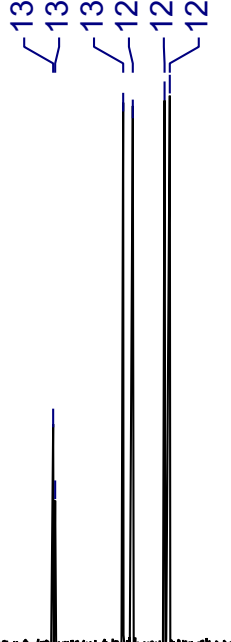

뉸ำ용

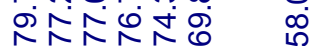

रU

:

จุ

लं

\begin{tabular}{|c|c|c|c|c|c|c|c|c|c|c|c|c|c|c|c|c|c|c|c|c|c|c|}
\hline 220 & 210 & 200 & 190 & 180 & 170 & 160 & 150 & 140 & 130 & 120 & $\begin{array}{l}110 \text { S61 } 100 \\
\text { f1 (ppm) }\end{array}$ & 90 & 80 & 70 & 60 & 50 & 40 & 30 & 20 & 10 & 0 & -10 \\
\hline
\end{tabular}




\begin{tabular}{|ll|}
\hline \multicolumn{1}{|c|}{ Parameter } & \multicolumn{1}{c|}{ Value } \\
1 Title & $\mathrm{xz}-1-\mathrm{SM}-282 \mathrm{BH}$ \\
2 Solvent & $\mathrm{CDCl} 3$ \\
3 Spectrometer Frequency & 499.86 \\
4 Nucleus & $1 \mathrm{H}$ \\
\hline
\end{tabular}
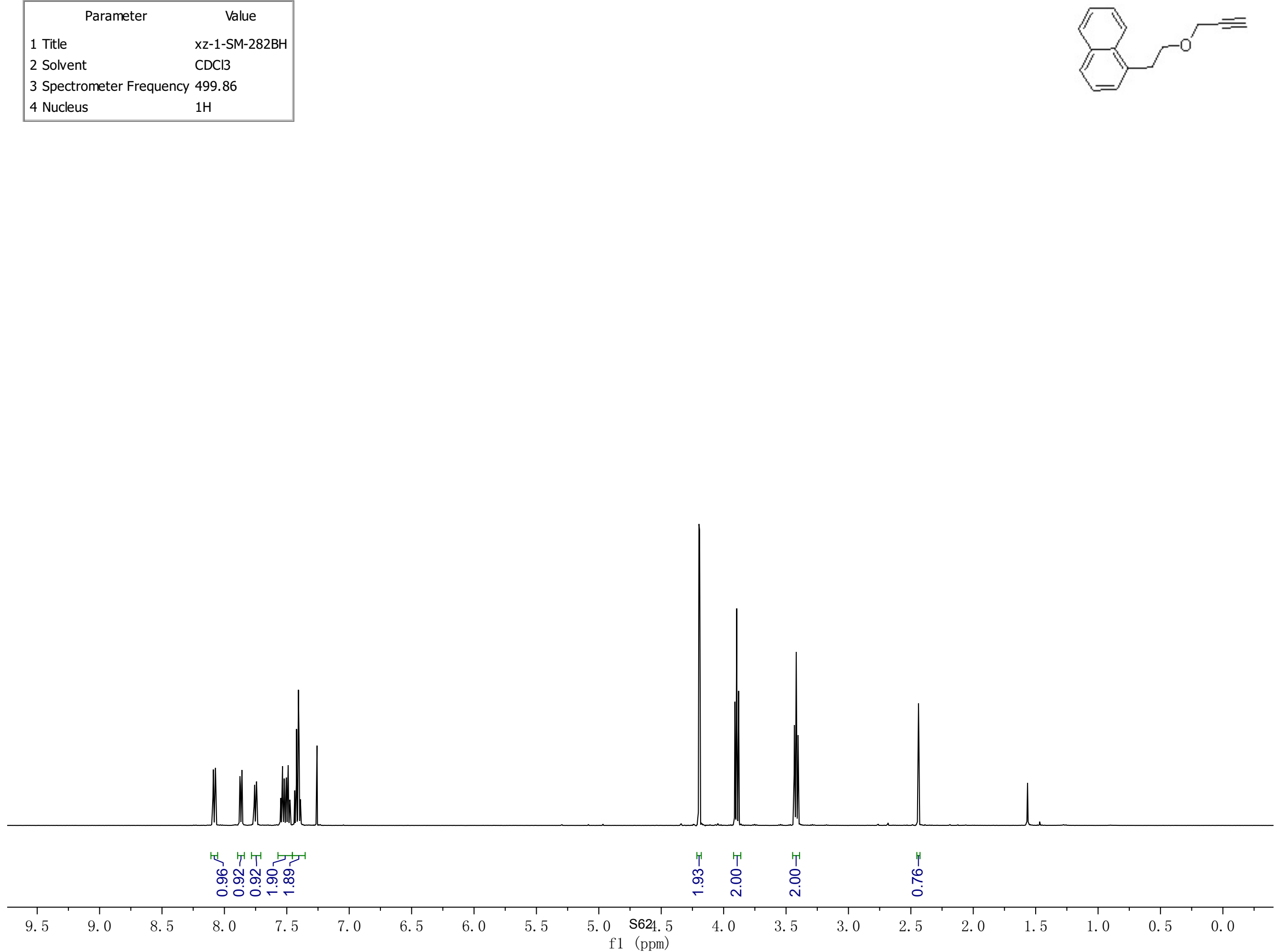


\begin{tabular}{|ll|}
\hline \multicolumn{1}{|c|}{ Parameter } & \multicolumn{1}{c|}{ Value } \\
1 Title & $\mathrm{xz}-1-\mathrm{SM}-282 \mathrm{BC}$ \\
2 Solvent & $\mathrm{CDCl} 3$ \\
3 Spectrometer Frequency & 125.70 \\
4 Nucleus & $13 \mathrm{C}$ \\
\hline
\end{tabular}

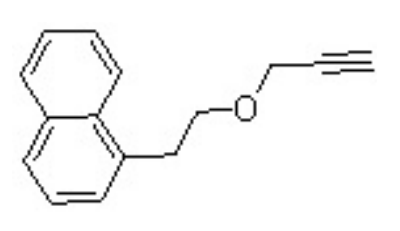

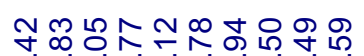

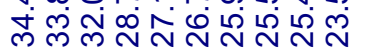

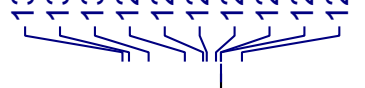

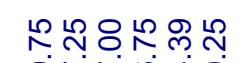

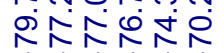

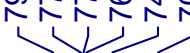

$\stackrel{\infty}{\circ}$

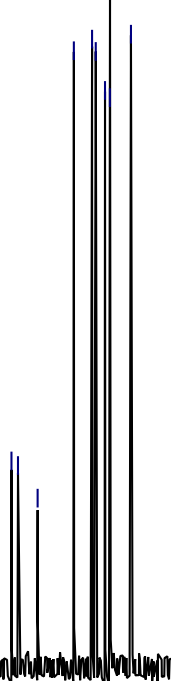

(1)

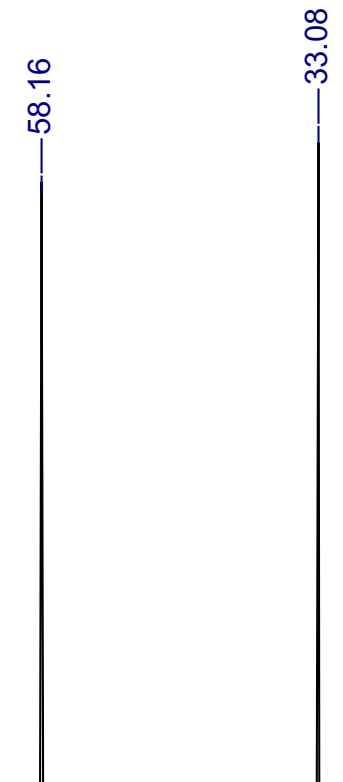

$\stackrel{\infty}{m}$

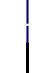

\begin{tabular}{|c|c|c|c|c|c|c|c|c|c|c|c|c|c|c|c|c|c|c|c|c|c|c|}
\hline 220 & 210 & 200 & $\begin{array}{l}1 \\
190\end{array}$ & 180 & $\begin{array}{l}1 \\
170\end{array}$ & 160 & 150 & 140 & 130 & $\begin{array}{c}1 \\
120\end{array}$ & $\begin{array}{l}110563100 \\
\text { f1 (ppm) }\end{array}$ & $\begin{array}{l}1 \\
90\end{array}$ & 80 & $\begin{array}{c}1 \\
70\end{array}$ & 60 & 50 & 40 & $\begin{array}{l}1 \\
30\end{array}$ & 20 & 10 & 0 & -10 \\
\hline
\end{tabular}




\begin{tabular}{|ll|}
\hline \multicolumn{1}{|c|}{ Parameter } & \multicolumn{1}{c|}{ Value } \\
1 Title & $\mathrm{xz}-1-283$ Bpure \\
2 Solvent & $\mathrm{CDCl} 3$ \\
3 Spectrometer Frequency & 499.86 \\
4 Nucleus & $1 \mathrm{H}$ \\
\hline
\end{tabular}
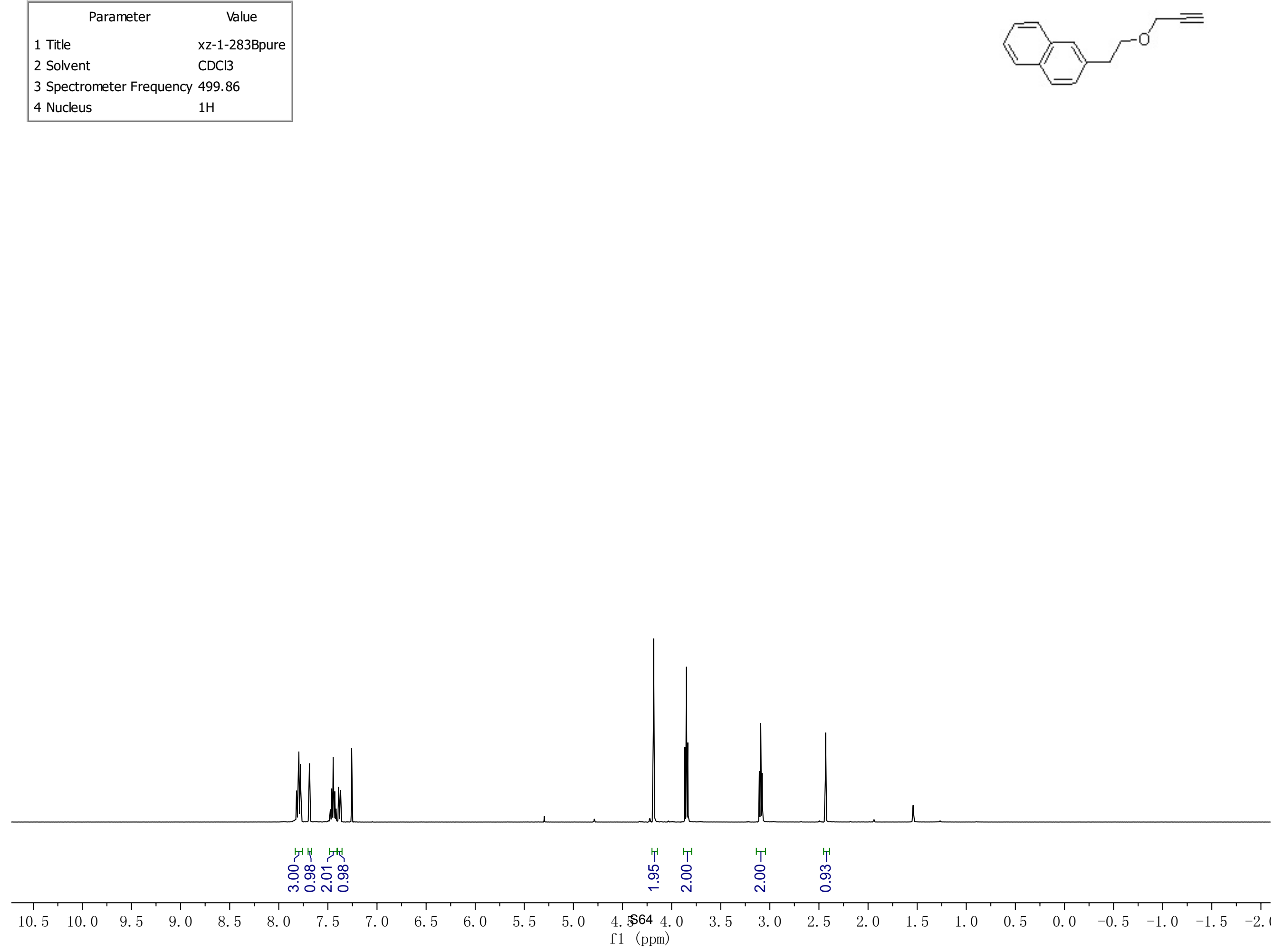


\begin{tabular}{|ll|}
\hline \multicolumn{1}{|c|}{ Parameter } & \multicolumn{1}{c|}{ Value } \\
1 Title & $\mathrm{xz}-1-283 \mathrm{BC500biaopu}$ \\
2 Solvent & $\mathrm{CDCl} 3$ \\
3 Spectrometer Frequency & 125.70 \\
4 Nucleus & $13 \mathrm{C}$ \\
\hline
\end{tabular}
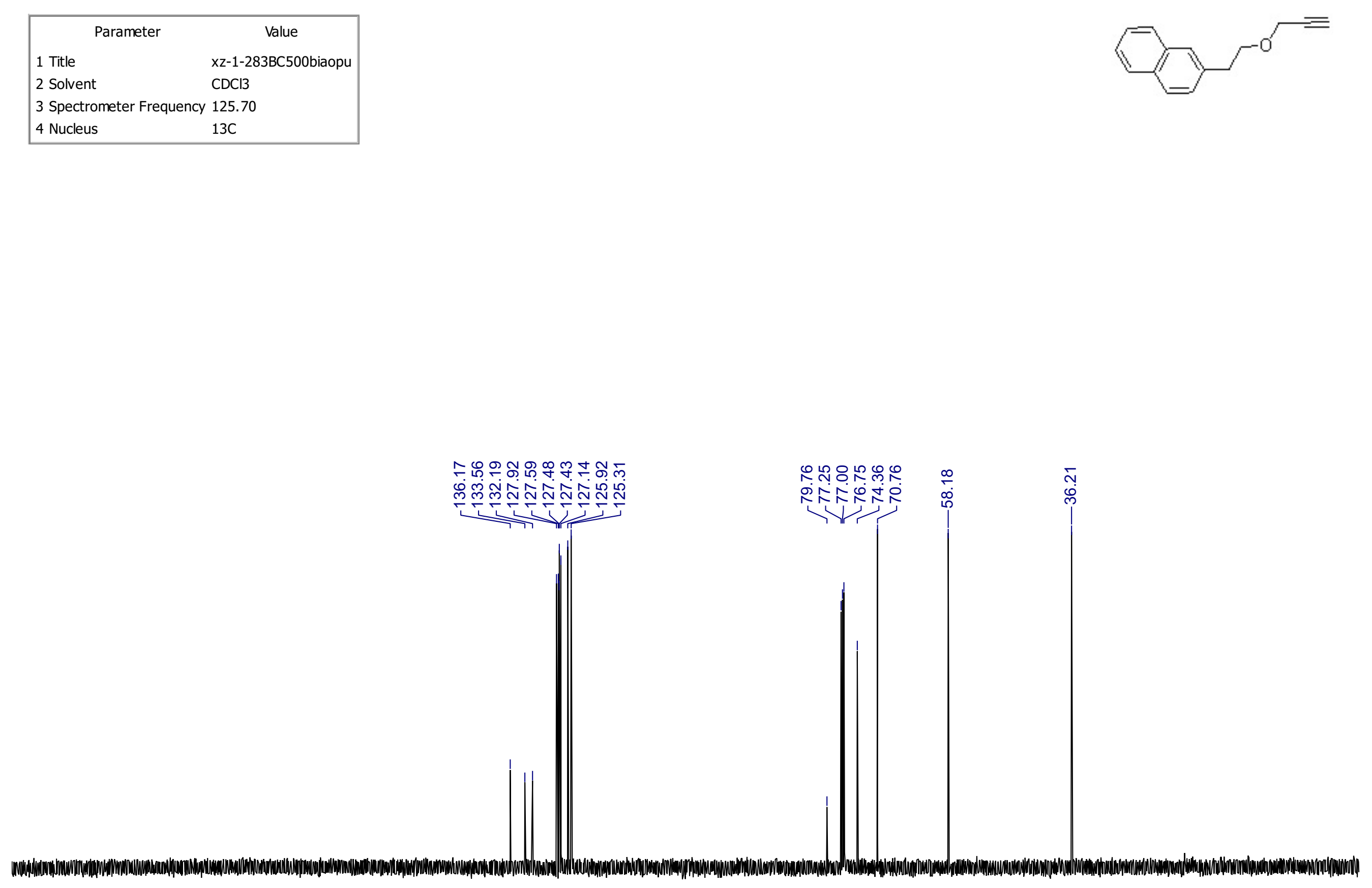

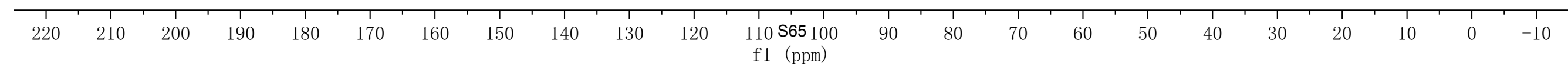




\begin{tabular}{|ll|}
\hline \multicolumn{1}{|c|}{ Parameter } & \multicolumn{1}{c|}{ Value } \\
1 Title & chy-2-294-2-SM-1H \\
2 Solvent & $\mathrm{CDCl} 3$ \\
3 Spectrometer Frequency & 499.86 \\
4 Nucleus & $1 \mathrm{H}$ \\
\hline
\end{tabular}
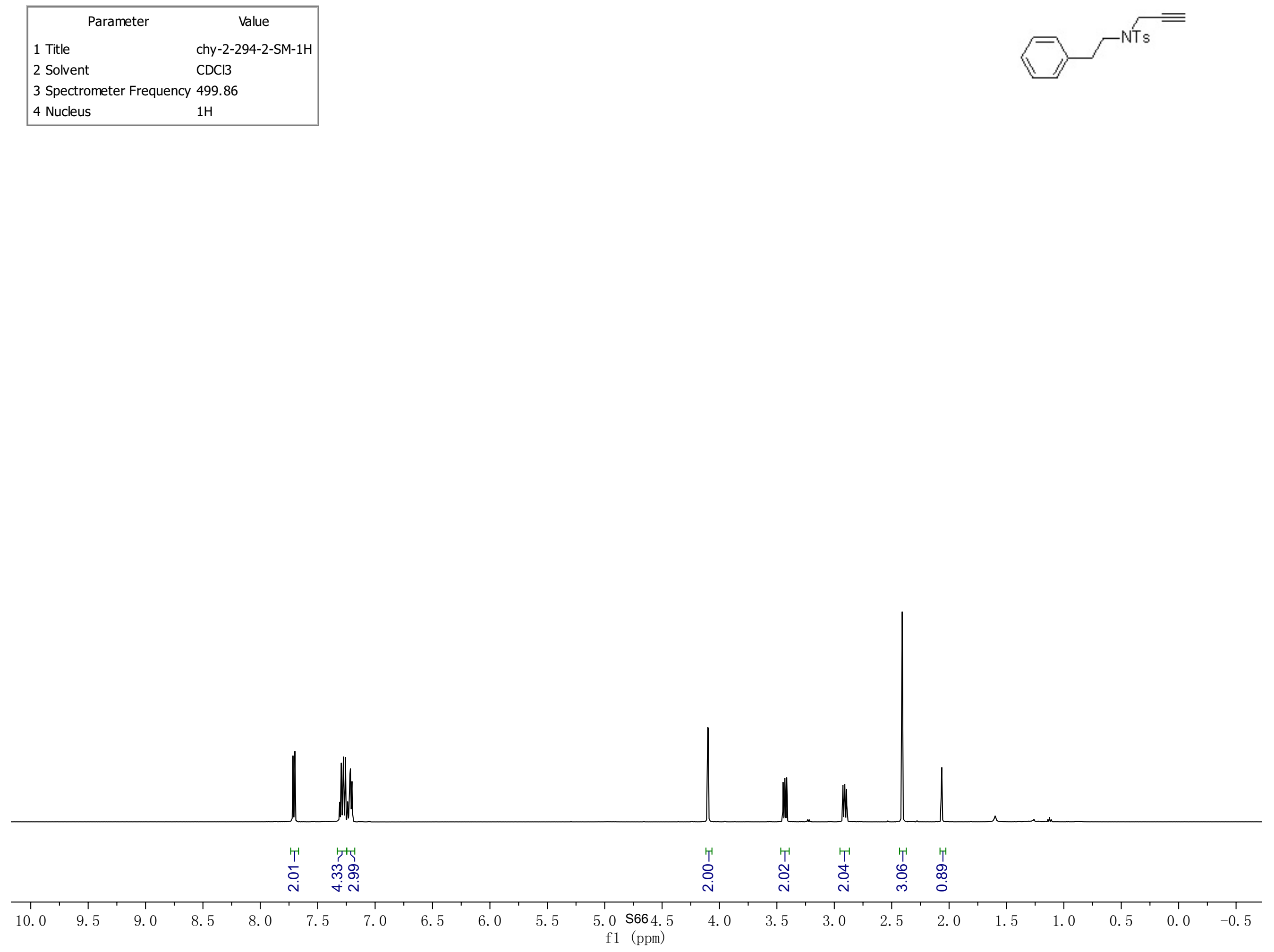


\begin{tabular}{|ll|}
\hline \multicolumn{1}{|c|}{ Parameter } & \multicolumn{1}{c|}{ Value } \\
1 Title & chy-2-294-2-SM-13C \\
2 Solvent & CDCl3 \\
3 Spectrometer Frequency & 125.70 \\
4 Nucleus & $13 \mathrm{C}$ \\
\hline
\end{tabular}
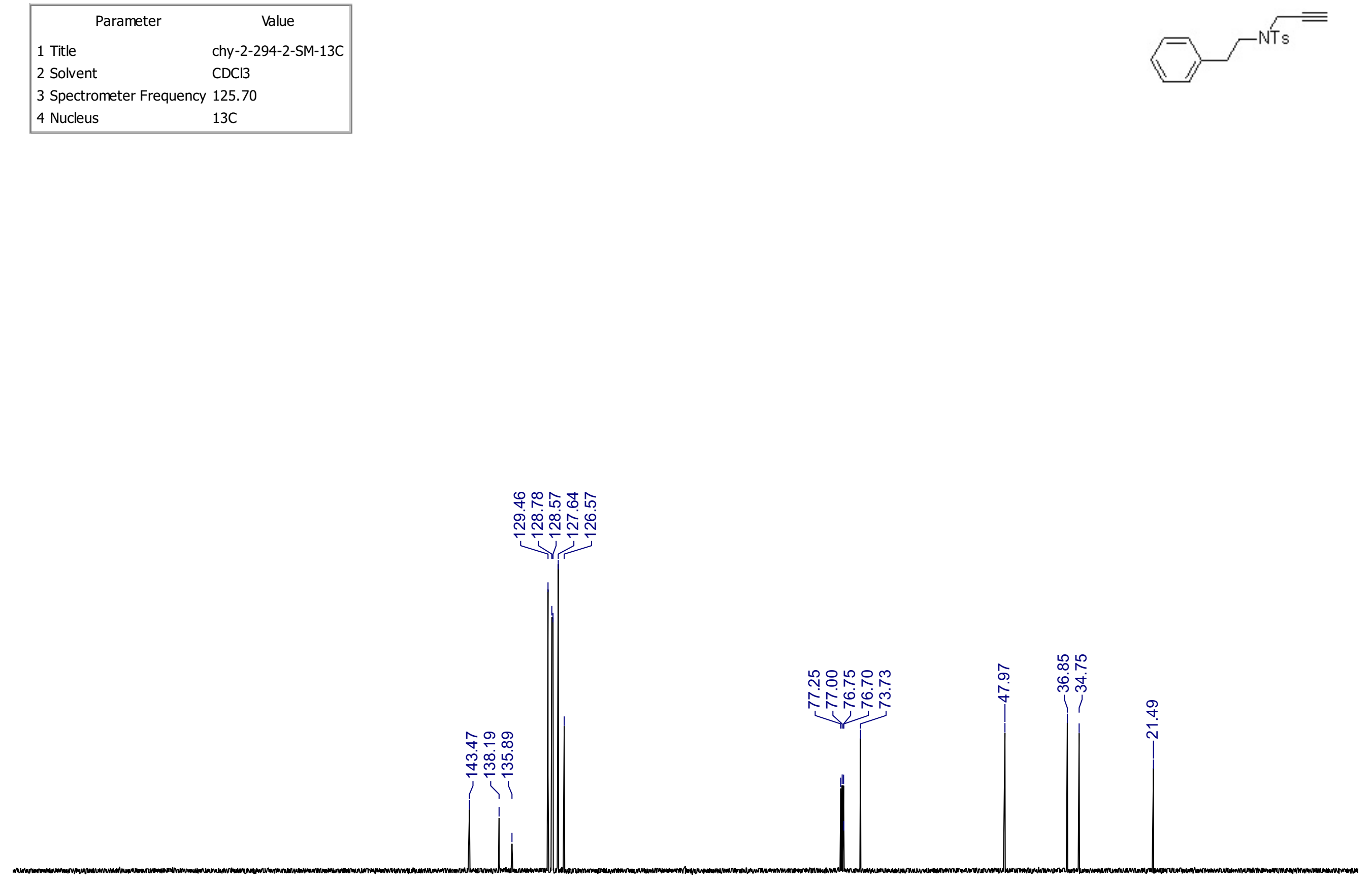

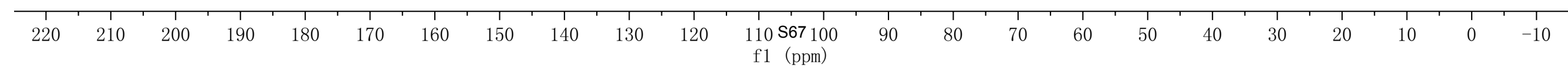




\begin{tabular}{|ll|}
\hline \multicolumn{1}{|c|}{ Parameter } & \multicolumn{1}{c|}{ Value } \\
1 Title & chy-3-144-1-SM-1H \\
2 Solvent & CDCl3 \\
3 Spectrometer Frequency & 499.86 \\
4 Nucleus & $1 \mathrm{H}$ \\
\hline
\end{tabular}
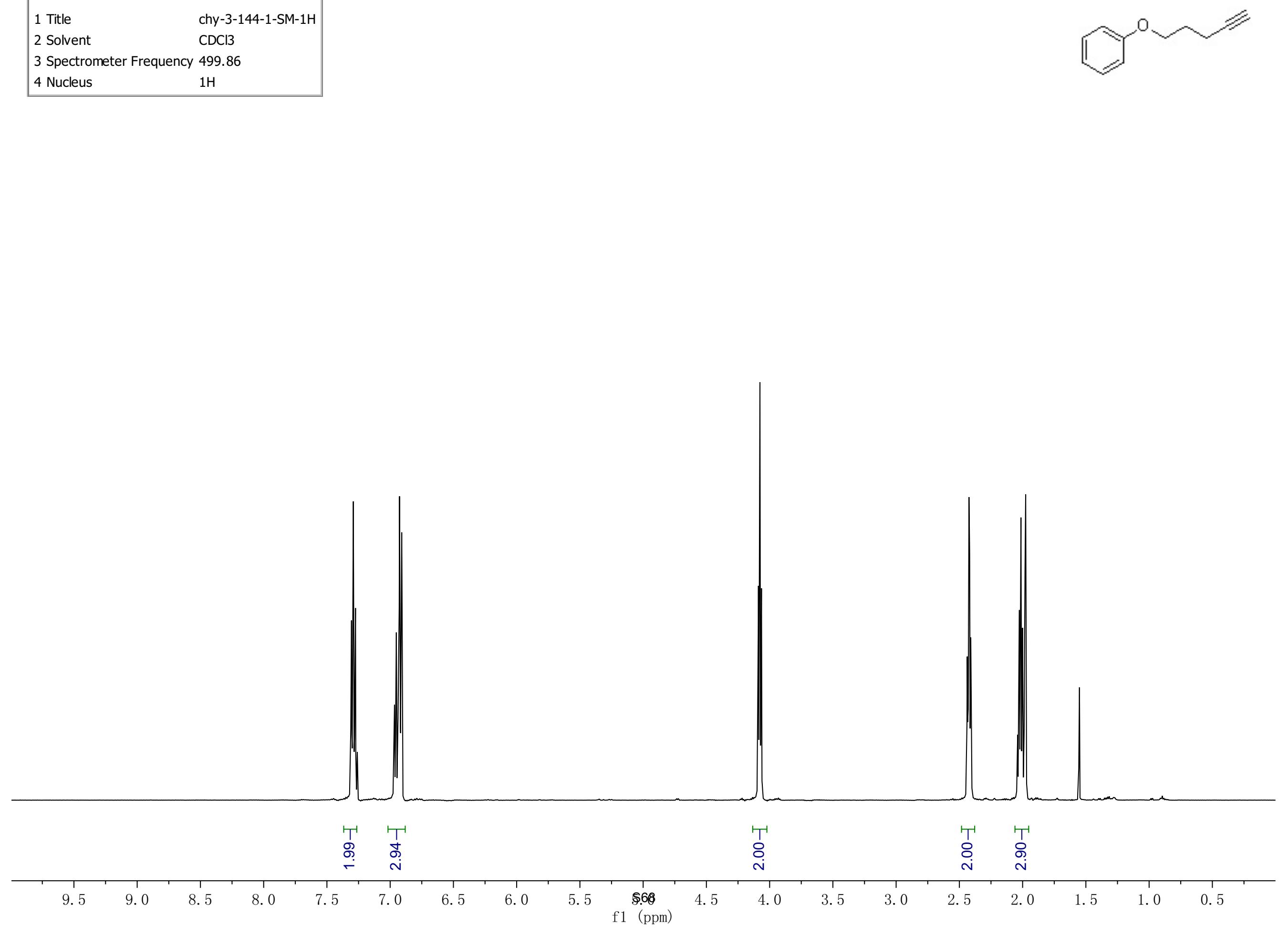


\begin{tabular}{|ll|}
\hline \multicolumn{1}{|c|}{ Parameter } & \multicolumn{1}{c|}{ Value } \\
1 Title & chy-3-144-1-SM-13C \\
2 Solvent & CDCl3 \\
3 Spectrometer Frequency & 125.70 \\
4 Nucleus & $13 \mathrm{C}$ \\
\hline
\end{tabular}
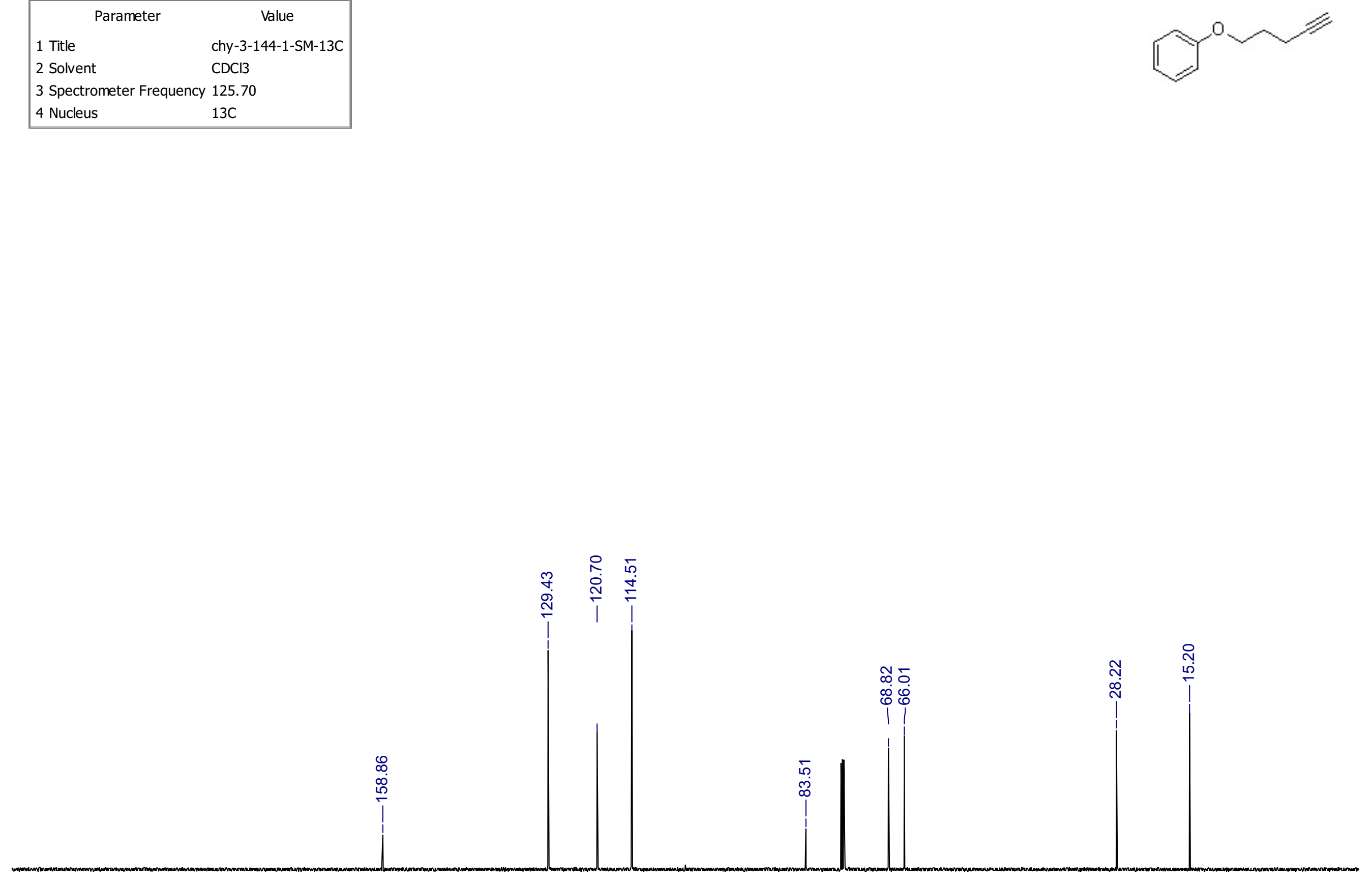

\begin{tabular}{|c|c|c|c|c|c|c|c|c|c|c|c|c|c|c|c|c|c|c|c|c|c|c|}
\hline 220 & 210 & 200 & 190 & 180 & 170 & 160 & 150 & 140 & $\begin{array}{c}1 \\
130\end{array}$ & 120 & $\begin{array}{l}110 \text { S69 } 100 \\
\text { f1 (ppm) }\end{array}$ & 90 & 80 & 70 & 60 & 50 & 40 & 30 & 20 & 10 & 0 & $\begin{array}{c}1 \\
-10\end{array}$ \\
\hline
\end{tabular}




\begin{tabular}{|ll|}
\hline \multicolumn{1}{|c|}{ Parameter } & \multicolumn{1}{c|}{ Value } \\
1 Title & $\mathrm{xz}-1-235 \mathrm{e} 500 \mathrm{H}$ \\
2 Solvent & $\mathrm{CDCl} 3$ \\
3 Spectrometer Frequency & 499.86 \\
4 Nucleus & $1 \mathrm{H}$ \\
\hline
\end{tabular}
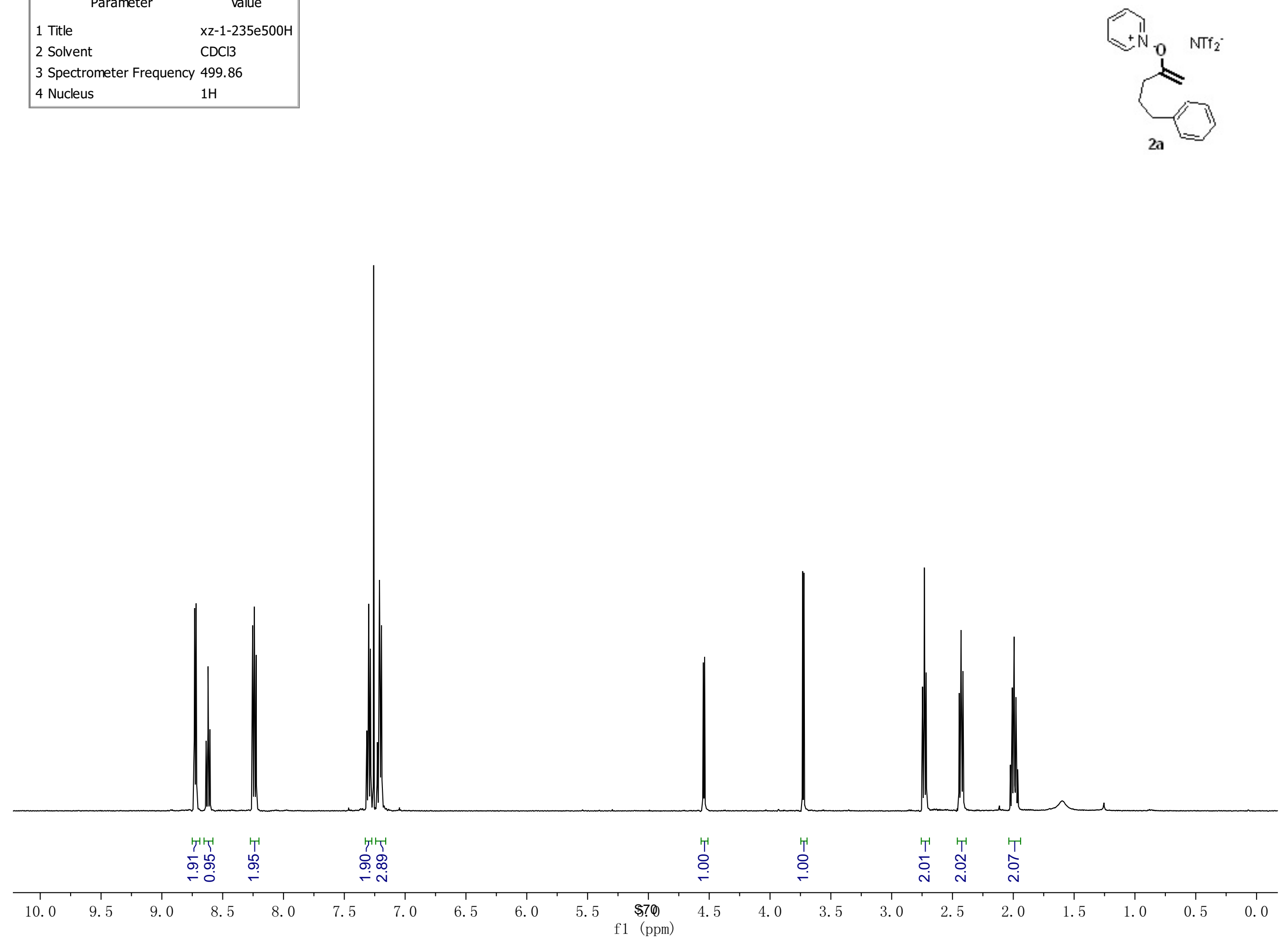


\begin{tabular}{|ll|}
\hline \multicolumn{1}{|c|}{ Parameter } & \multicolumn{1}{c|}{ Value } \\
1 Title & $\mathrm{xz}-1-235$ ebiaopuC500 \\
2 Solvent & $\mathrm{CDCl} 3$ \\
3 Spectrometer Frequency & 125.70 \\
4 Nucleus & $13 \mathrm{C}$ \\
\hline
\end{tabular}

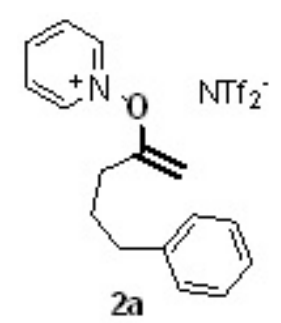

뜌유요

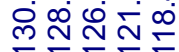

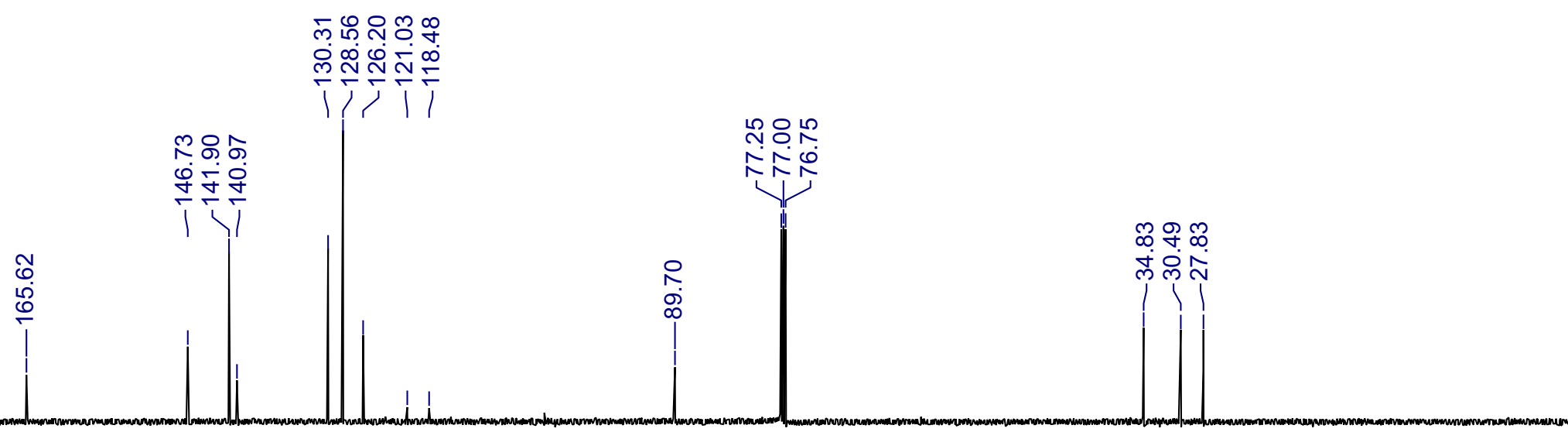

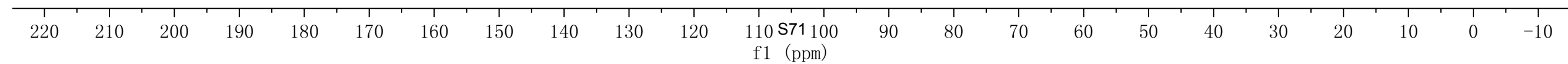




\begin{tabular}{|ll|}
\hline \multicolumn{1}{|c|}{ Parameter } & \multicolumn{1}{c|}{ Value } \\
1 Title & $\mathrm{xz}-1-199 \mathrm{~A} 2 \mathrm{standardH}$ \\
2 Solvent & $\mathrm{CDCl} 3$ \\
3 Spectrometer Frequency & 499.86 \\
4 Nucleus & $1 \mathrm{H}$ \\
\hline
\end{tabular}

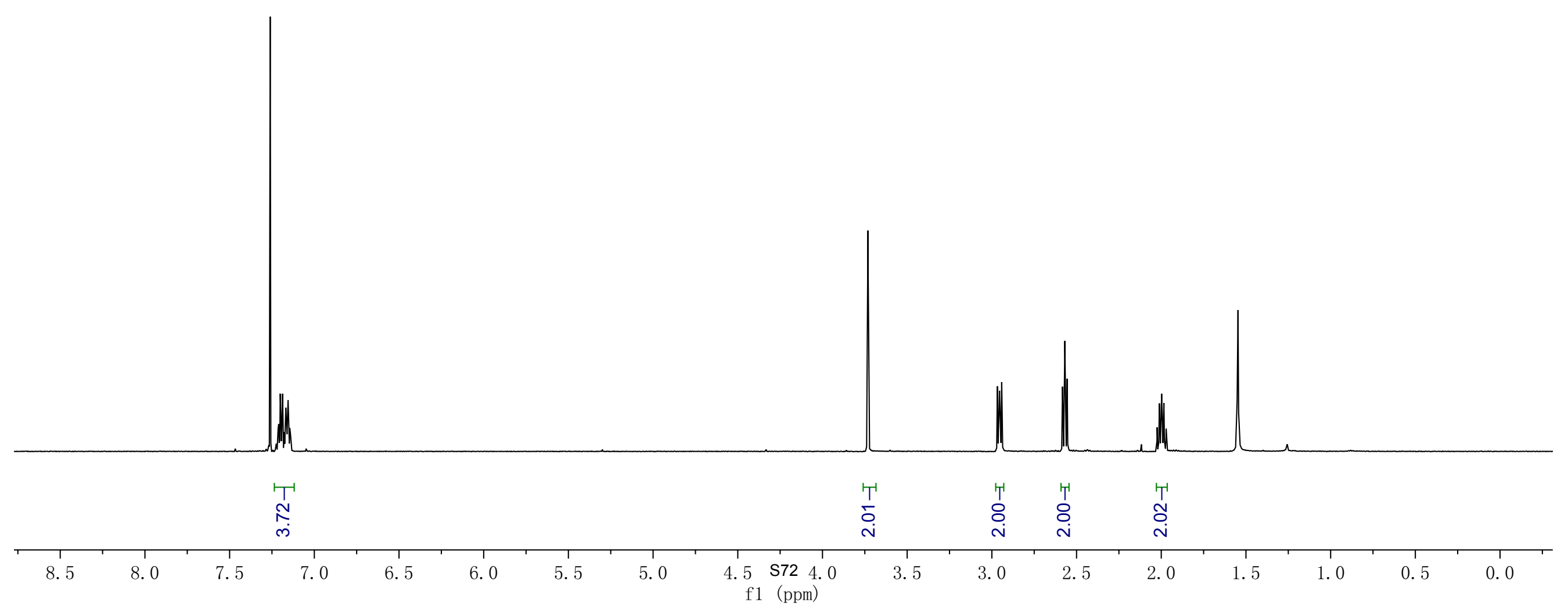




\begin{tabular}{|ll|}
\hline \multicolumn{1}{|c|}{ Parameter } & \multicolumn{1}{c|}{ Value } \\
1 Title & $\mathrm{xz}-1-199 \mathrm{~A} 2 \mathrm{C} 3$ \\
2 Solvent & $\mathrm{CDCl} 3$ \\
3 Spectrometer Frequency & 125.70 \\
4 Nucleus & $13 \mathrm{C}$ \\
\hline
\end{tabular}

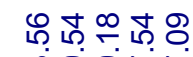

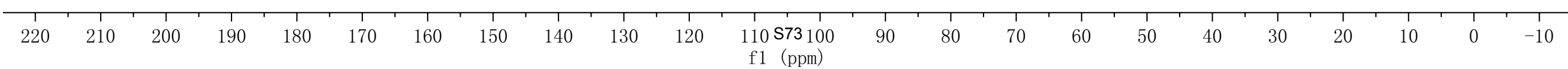




\begin{tabular}{|ll|}
\hline \multicolumn{1}{|c|}{ Parameter } & \multicolumn{1}{c|}{ Value } \\
1 Title & $\mathrm{xz}-1-199 \mathrm{BH} 500$ \\
2 Solvent & $\mathrm{CDCl} 3$ \\
3 Spectrometer Frequency & 499.86 \\
4 Nucleus & $1 \mathrm{H}$ \\
\hline
\end{tabular}

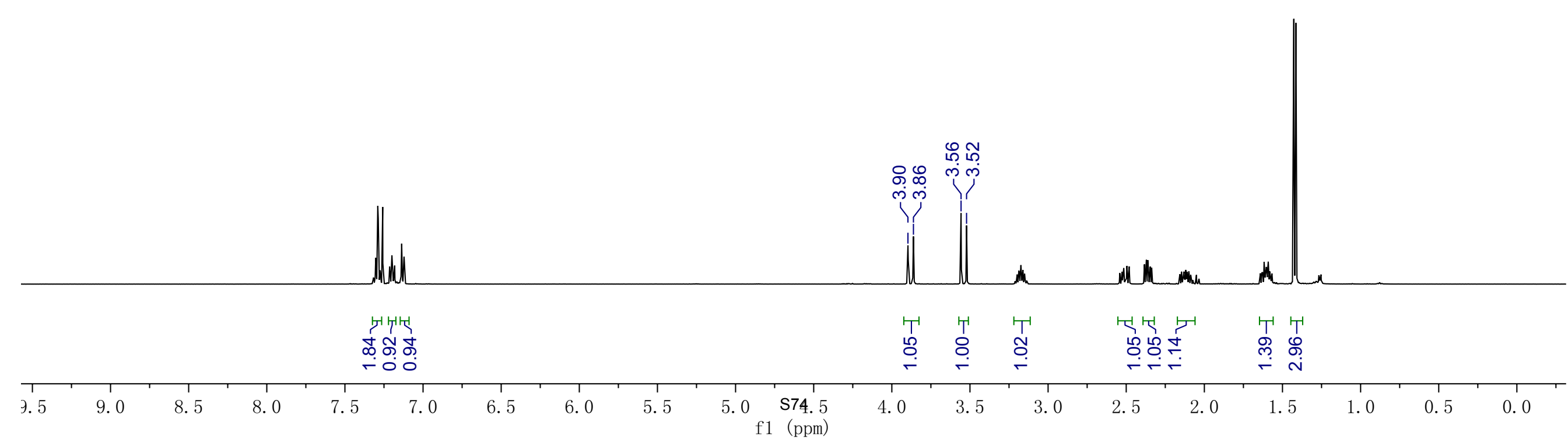




\begin{tabular}{|ll|}
\hline \multicolumn{1}{|c|}{ Parameter } & \multicolumn{1}{c|}{ Value } \\
1 Title & $\mathrm{xz}-1-199 \mathrm{~B} 500 \mathrm{C}$ \\
2 Solvent & $\mathrm{CDCl} 3$ \\
3 Spectrometer Frequency & 125.70 \\
4 Nucleus & $13 \mathrm{C}$ \\
\hline
\end{tabular}

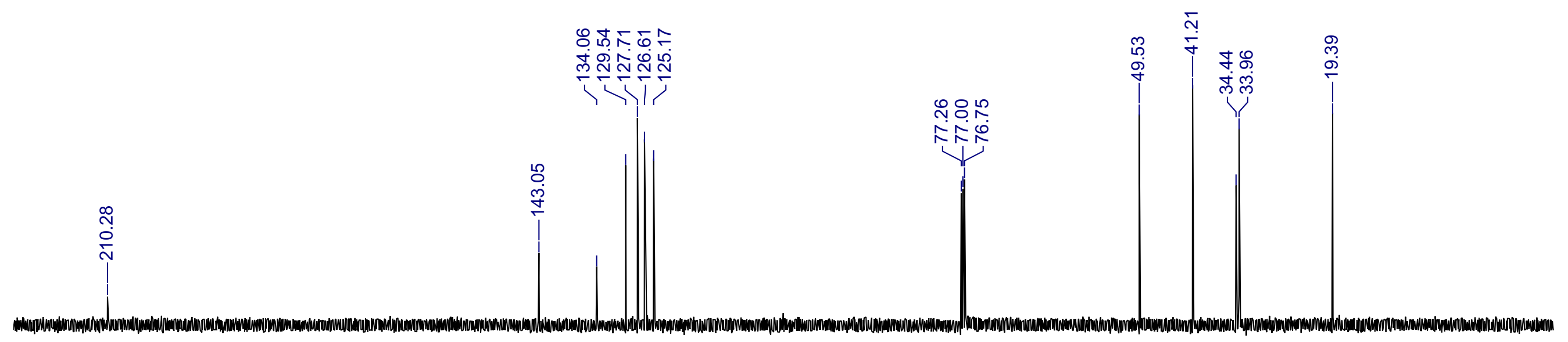

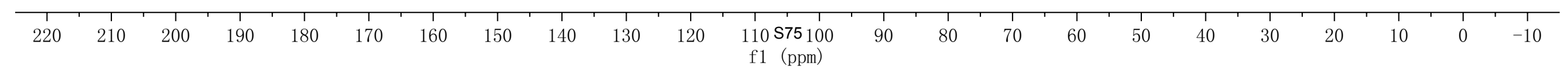




\begin{tabular}{|ll|}
\hline \multicolumn{1}{|c|}{ Parameter } & \multicolumn{1}{c|}{ Value } \\
1 Title & $\mathrm{xz}-1-199 \mathrm{C} 2 \mathrm{H} 500$ \\
2 Solvent & $\mathrm{CDCl} 3$ \\
3 Spectrometer Frequency & 499.86 \\
4 Nucleus & $1 \mathrm{H}$ \\
\hline
\end{tabular}

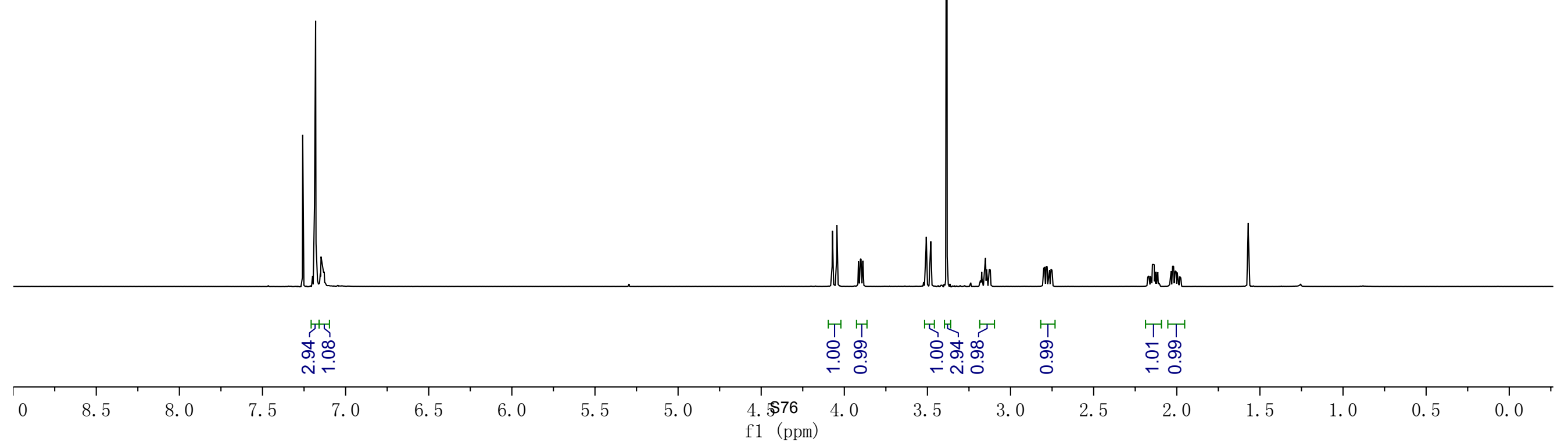




\begin{tabular}{|ll|}
\hline \multicolumn{1}{|c|}{ Parameter } & \multicolumn{1}{c|}{ Value } \\
1 Title & $\mathrm{xz}-1-199 \mathrm{C} 2500 \mathrm{C}$ \\
2 Solvent & $\mathrm{CDCl} 3$ \\
3 Spectrometer Frequency & 125.70 \\
4 Nucleus & $13 \mathrm{C}$ \\
\hline
\end{tabular}
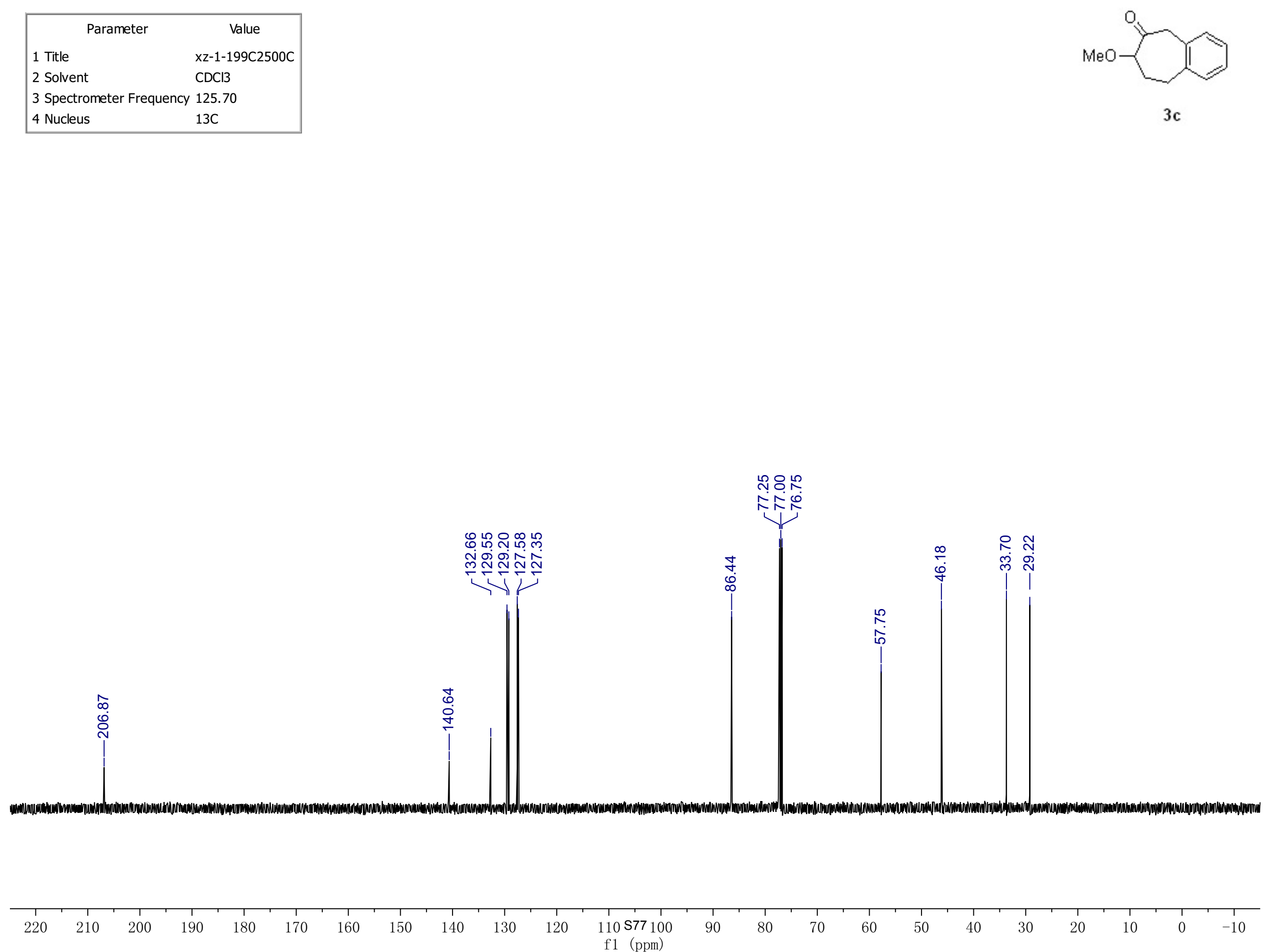


\begin{tabular}{|ll|}
\hline \multicolumn{1}{|c|}{ Parameter } & \multicolumn{1}{c|}{ Value } \\
1 Title & $\mathrm{xz}-1-213$ ebiaopu500H \\
2 Solvent & $\mathrm{CDCl} 3$ \\
3 Spectrometer Frequency & 499.86 \\
4 Nucleus & $1 \mathrm{H}$ \\
\hline
\end{tabular}
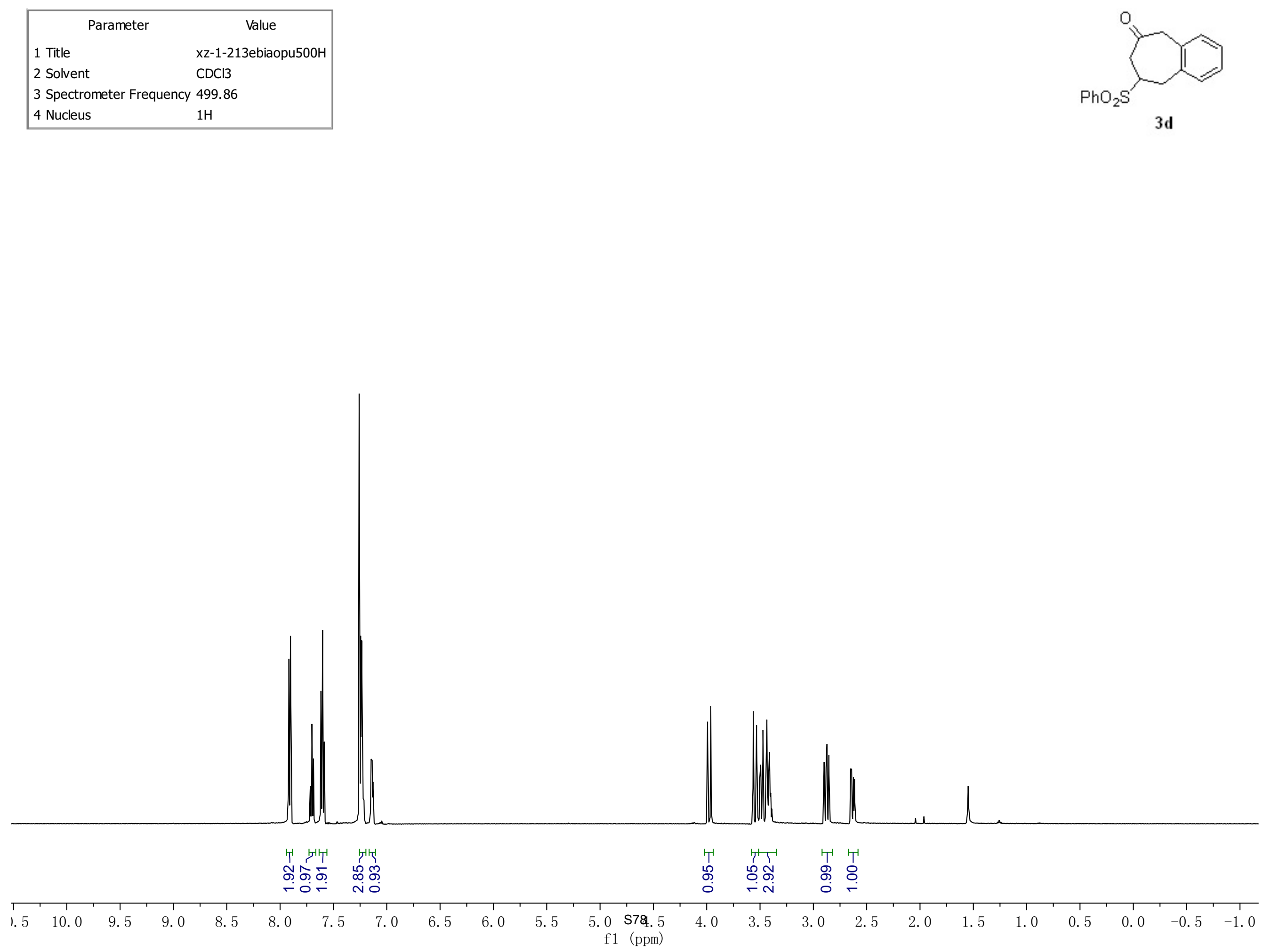


\begin{tabular}{|ll|}
\hline \multicolumn{1}{|c|}{ Parameter } & \multicolumn{1}{c|}{ Value } \\
1 Title & $\mathrm{xz}-1-213$ ebiaopu500C \\
2 Solvent & $\mathrm{CDCl} 3$ \\
3 Spectrometer Frequency & 125.70 \\
4 Nucleus & $13 \mathrm{C}$ \\
\hline
\end{tabular}
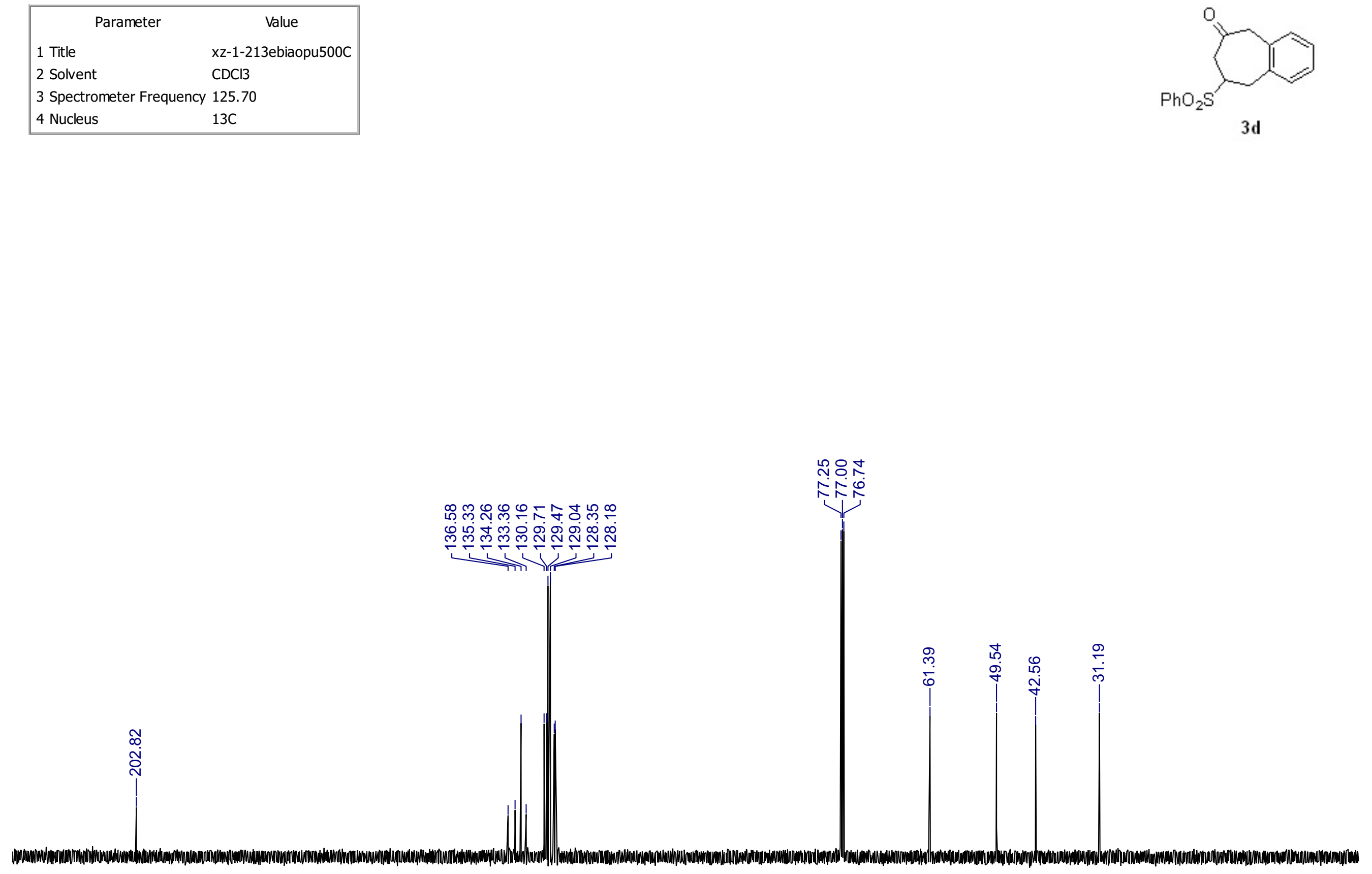

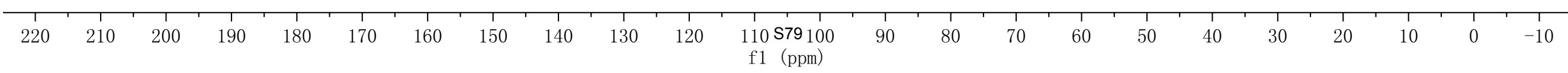




\begin{tabular}{|ll|}
\hline \multicolumn{1}{|c|}{ Parameter } & \multicolumn{1}{c|}{ Value } \\
1 Title & $\mathrm{xz}-1-205$ AH500biao \\
2 Solvent & $\mathrm{CDCl} 3$ \\
3 Spectrometer Frequency & 499.86 \\
4 Nucleus & $1 \mathrm{H}$ \\
\hline
\end{tabular}
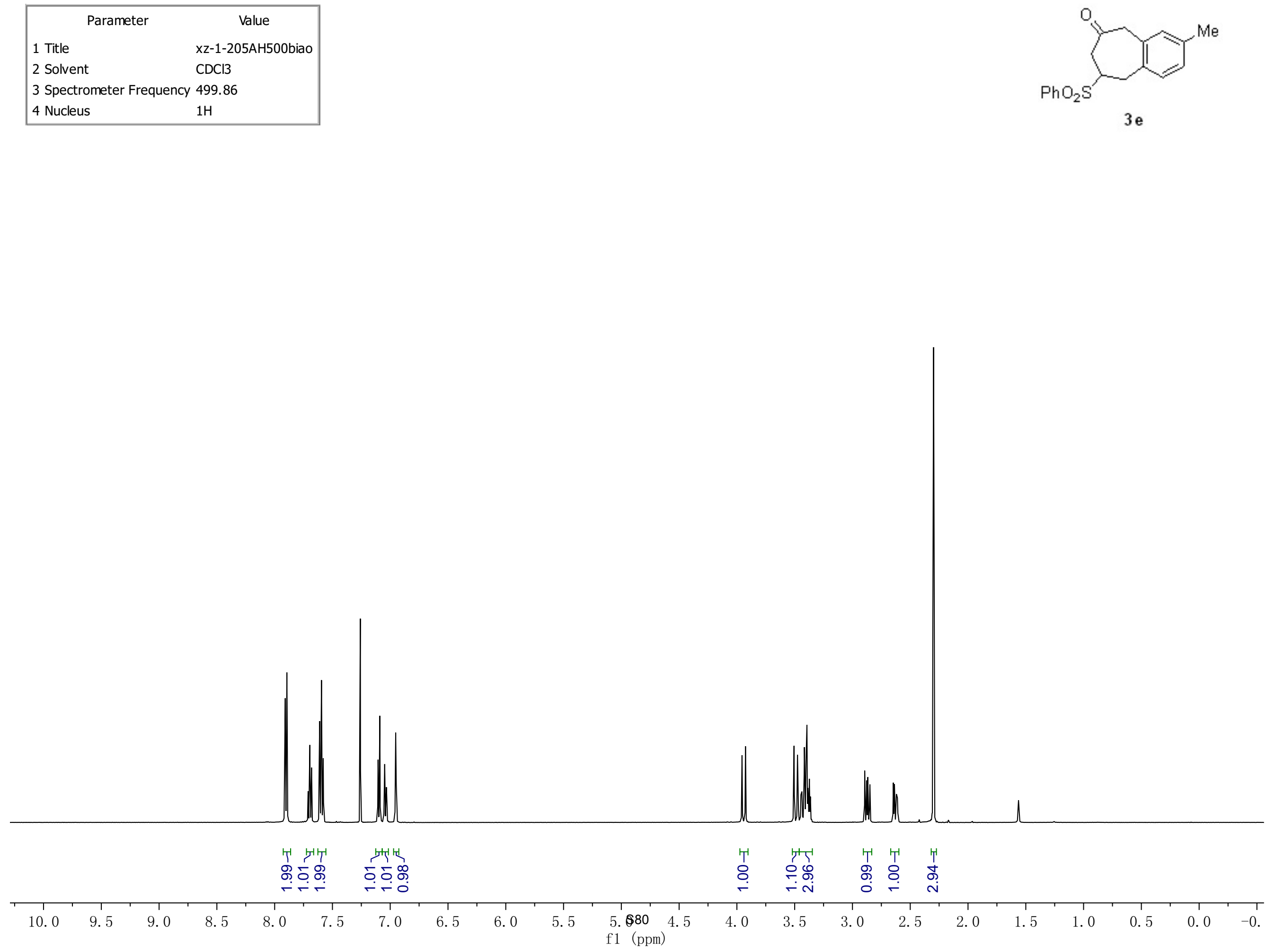


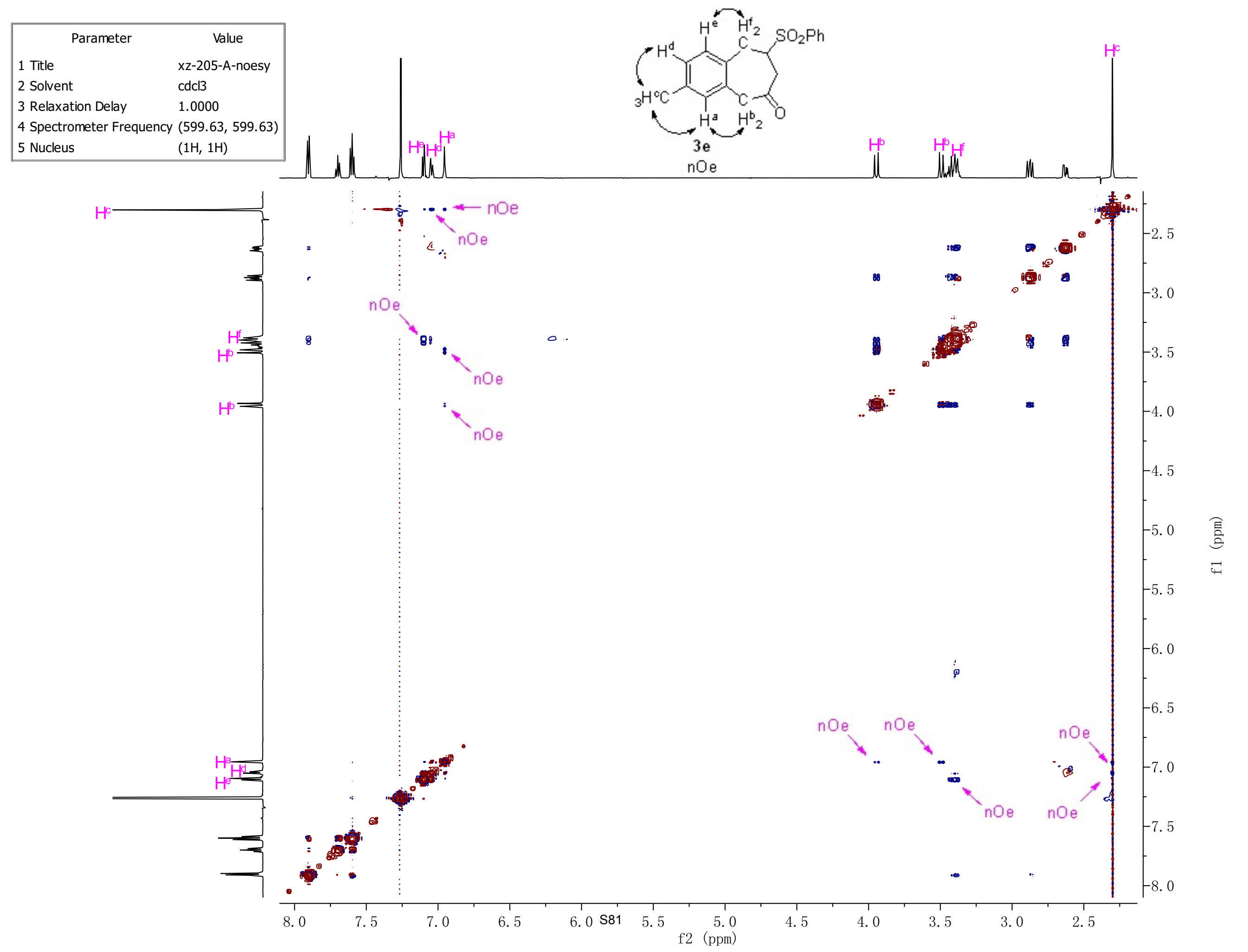




\begin{tabular}{|ll|}
\hline \multicolumn{1}{|c|}{ Parameter } & \multicolumn{1}{c|}{ Value } \\
1 Title & $\mathrm{xz}-1-205 \mathrm{AC} 500$ \\
2 Solvent & $\mathrm{CDCl} 3$ \\
3 Spectrometer Frequency & 125.70 \\
4 Nucleus & $13 \mathrm{C}$ \\
\hline
\end{tabular}
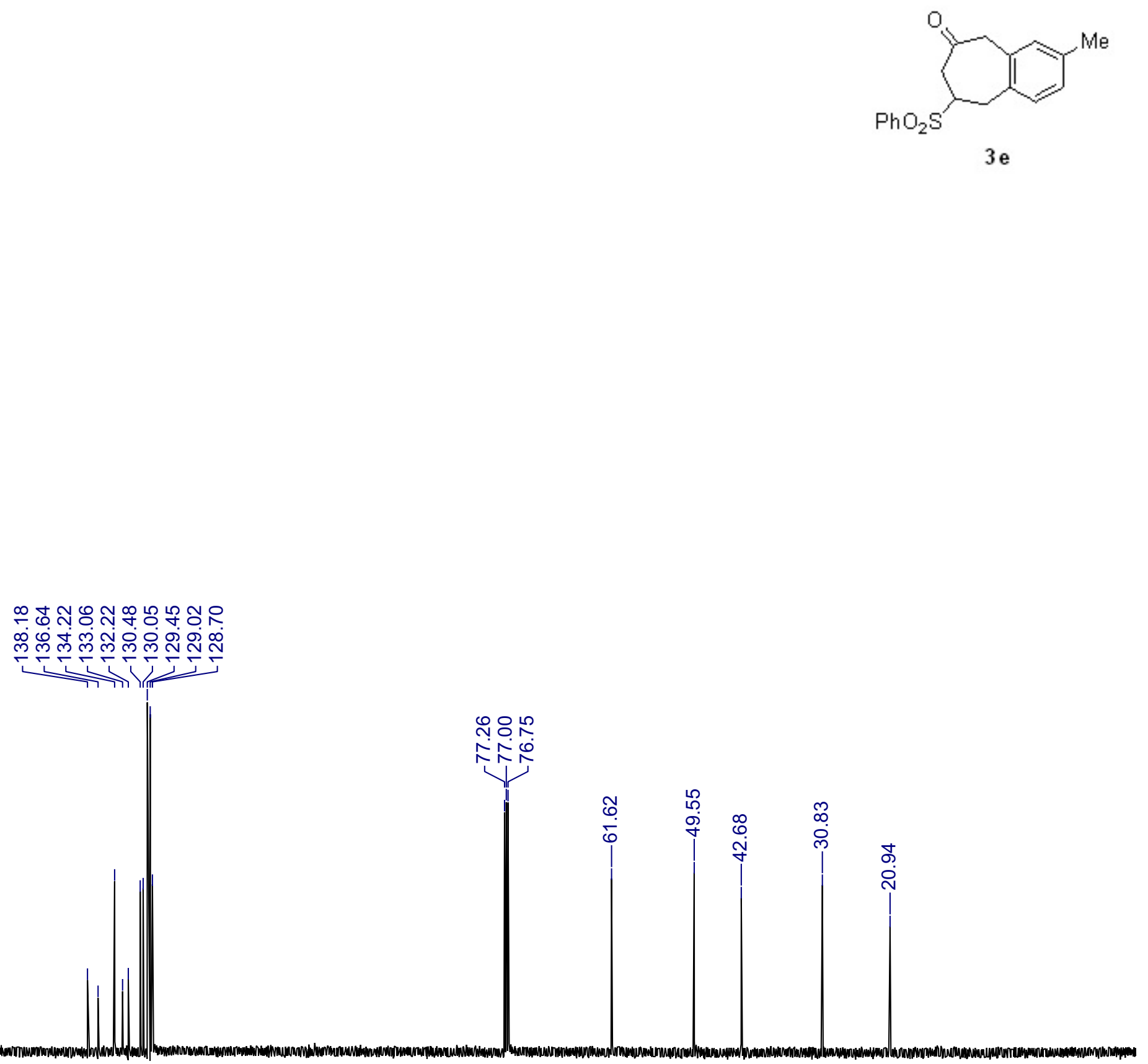

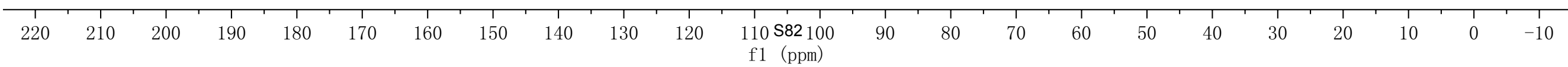




\begin{tabular}{|ll|}
\hline \multicolumn{1}{|c|}{ Parameter } & \multicolumn{1}{c|}{ Value } \\
1 Title & $\mathrm{xz}-1-212 \mathrm{Am}$ mixH-500 \\
2 Solvent & $\mathrm{CDCl} 3$ \\
3 Spectrometer Frequency & 499.86 \\
4 Nucleus & $1 \mathrm{H}$ \\
\hline
\end{tabular}
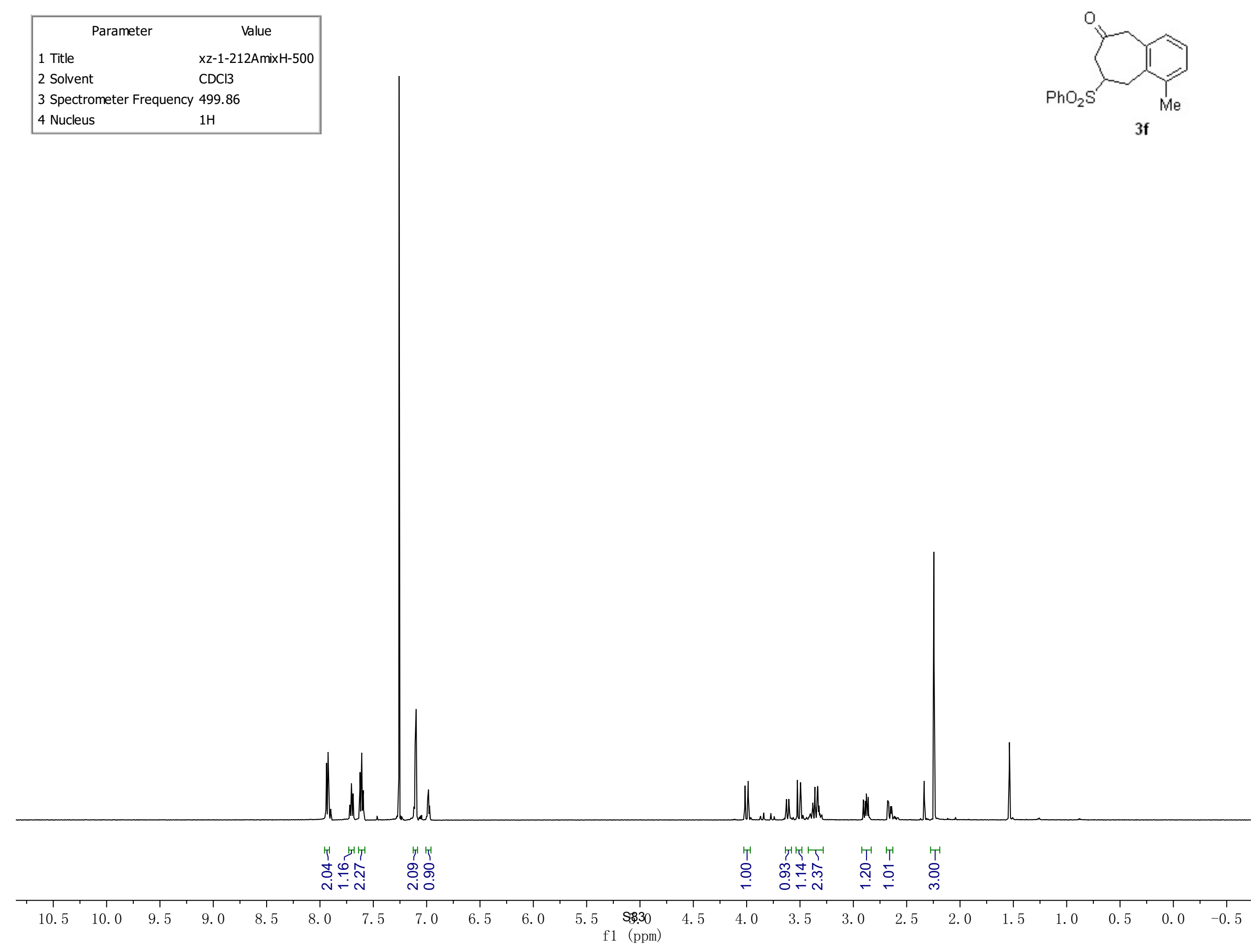


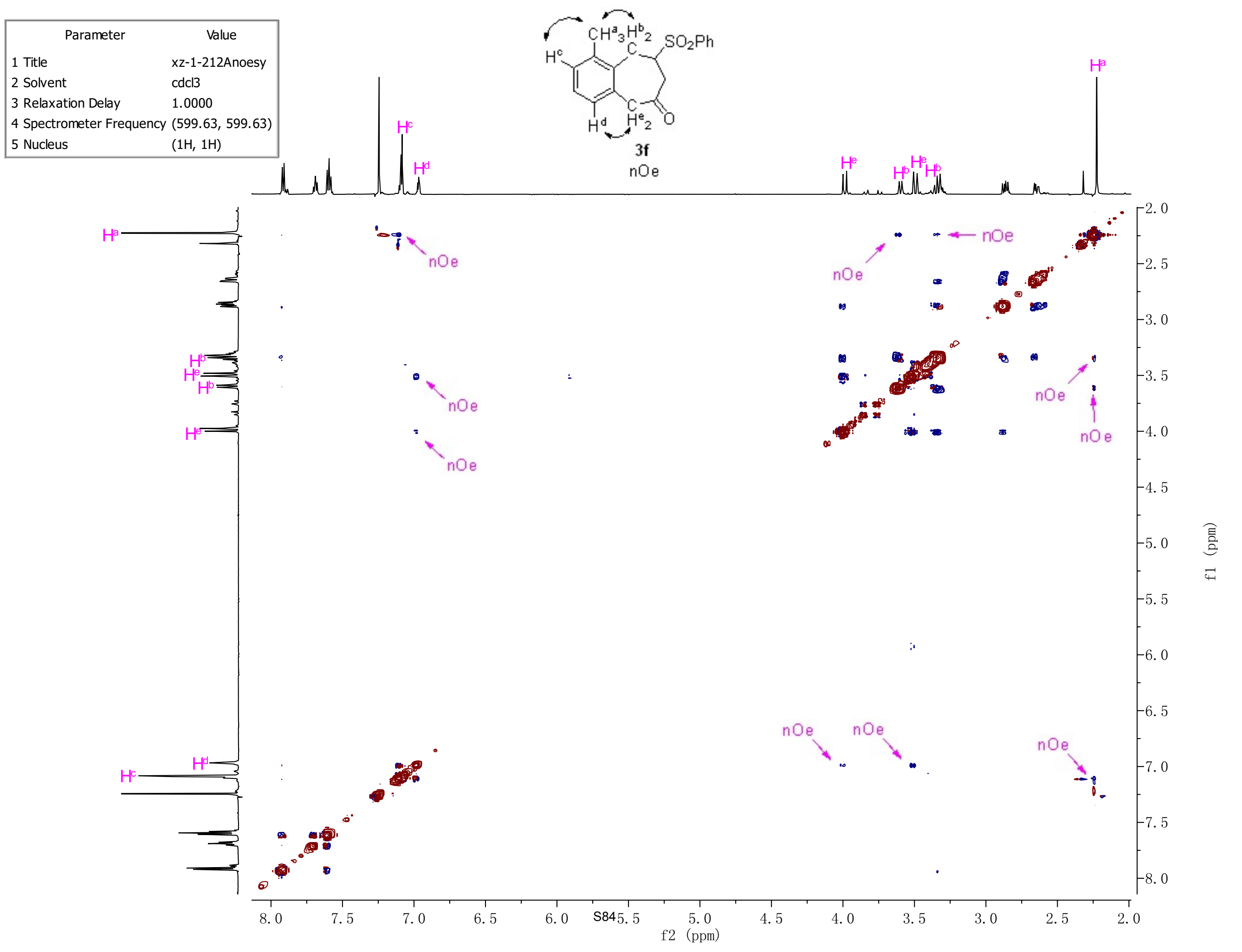




\begin{tabular}{|ll|}
\hline \multicolumn{1}{|c|}{ Parameter } & \multicolumn{1}{c|}{ Value } \\
1 Title & $\mathrm{xz}-1-212 \mathrm{ACmix}-500$ \\
2 Solvent & $\mathrm{CDCl} 3$ \\
3 Spectrometer Frequency & 125.70 \\
4 Nucleus & $13 \mathrm{C}$ \\
\hline
\end{tabular}
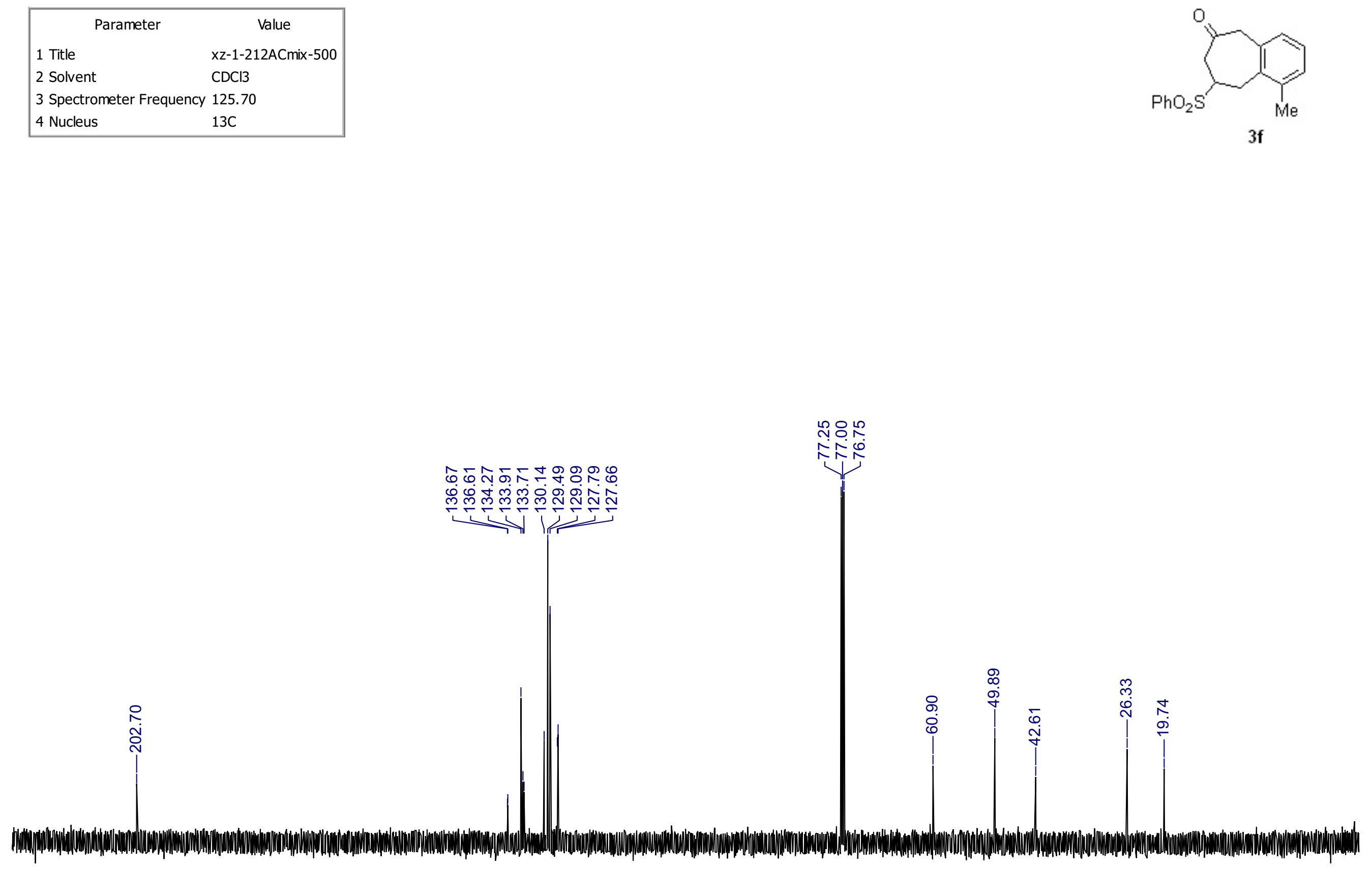

\begin{tabular}{|c|c|c|c|c|c|c|c|c|c|c|c|c|c|c|c|c|c|c|c|c|c|c|}
\hline $\begin{array}{l}1 \\
220\end{array}$ & 210 & $\begin{array}{l}1 \\
200\end{array}$ & $\begin{array}{c}1 \\
190\end{array}$ & $\begin{array}{l}1 \\
180\end{array}$ & $\begin{array}{ll}1 \\
170\end{array}$ & $\begin{array}{c}1 \\
160\end{array}$ & $\begin{array}{l}1 \\
150\end{array}$ & 14 & 130 & $\begin{array}{ll}1 \\
120\end{array}$ & 110 S85 100 & 90 & 80 & 70 & $\begin{array}{l}1 \\
60\end{array}$ & $\begin{array}{l}1 \\
50\end{array}$ & $\frac{1}{40}$ & $\begin{array}{l}1 \\
30\end{array}$ & 20 & 10 & $\begin{array}{l}1 \\
0\end{array}$ & $\begin{array}{l}1 \\
-10\end{array}$ \\
\hline & & & & & & & & & & & f1 (ppm) & 90 & 80 & 10 & & 50 & 40 & 30 & 20 & 10 & 0 & -10 \\
\hline
\end{tabular}




\begin{tabular}{|ll|}
\hline \multicolumn{1}{|c|}{ Parameter } & \multicolumn{1}{c|}{ Value } \\
1 Title & $\mathrm{xz}-1-264 \mathrm{BH} 500$ biaopu \\
2 Solvent & $\mathrm{CDCl} 3$ \\
3 Spectrometer Frequency & 499.86 \\
4 Nucleus & $1 \mathrm{H}$ \\
\hline
\end{tabular}

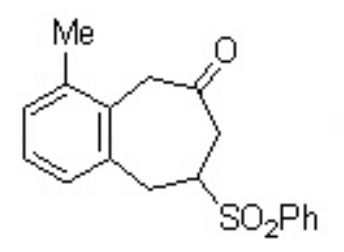

$3 \mathrm{~g}$

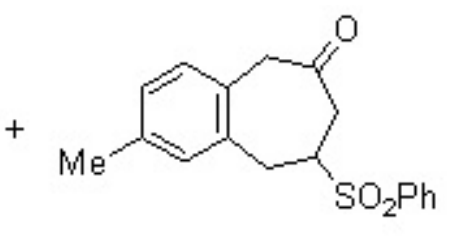

$3 \mathrm{~g}^{\circ}$

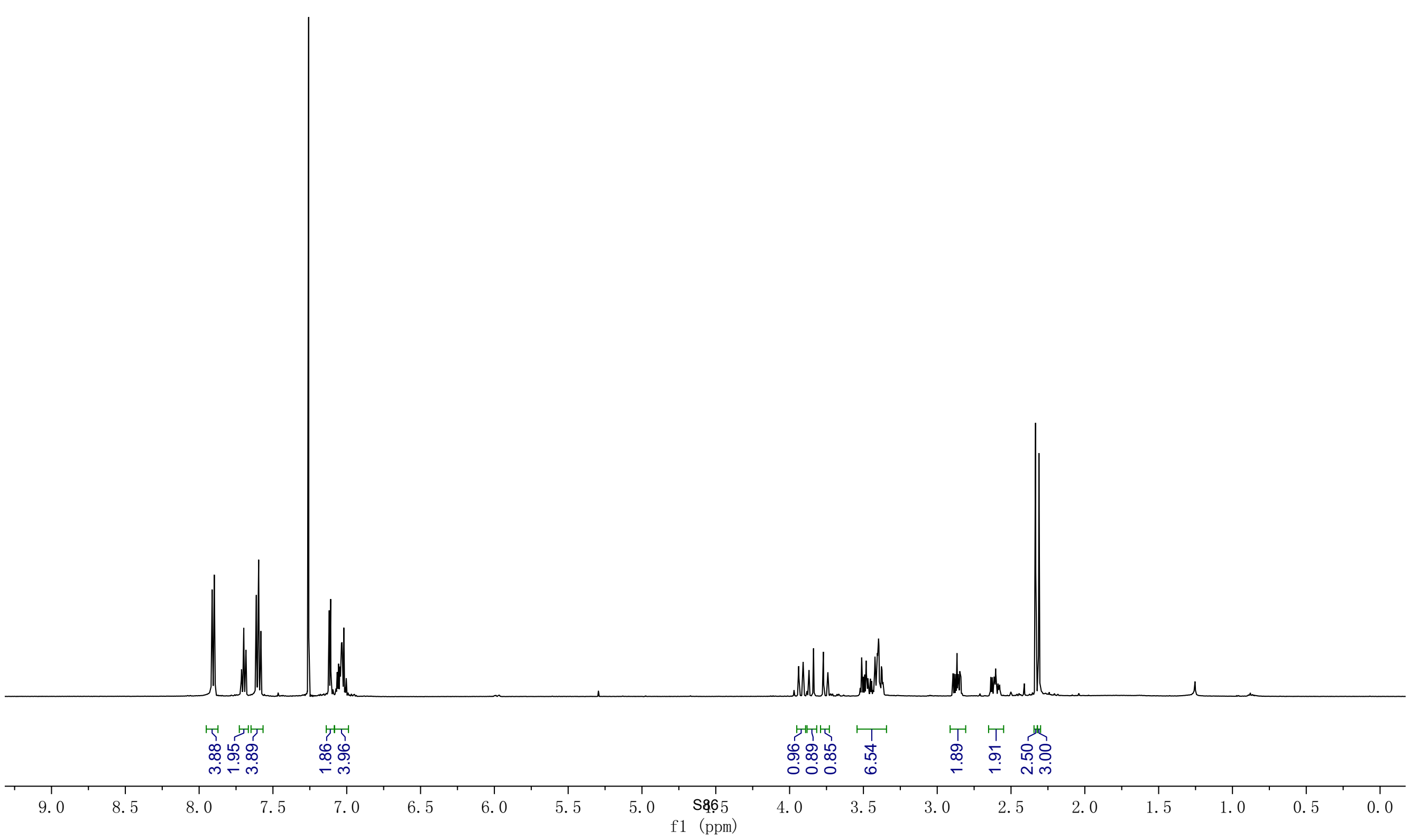




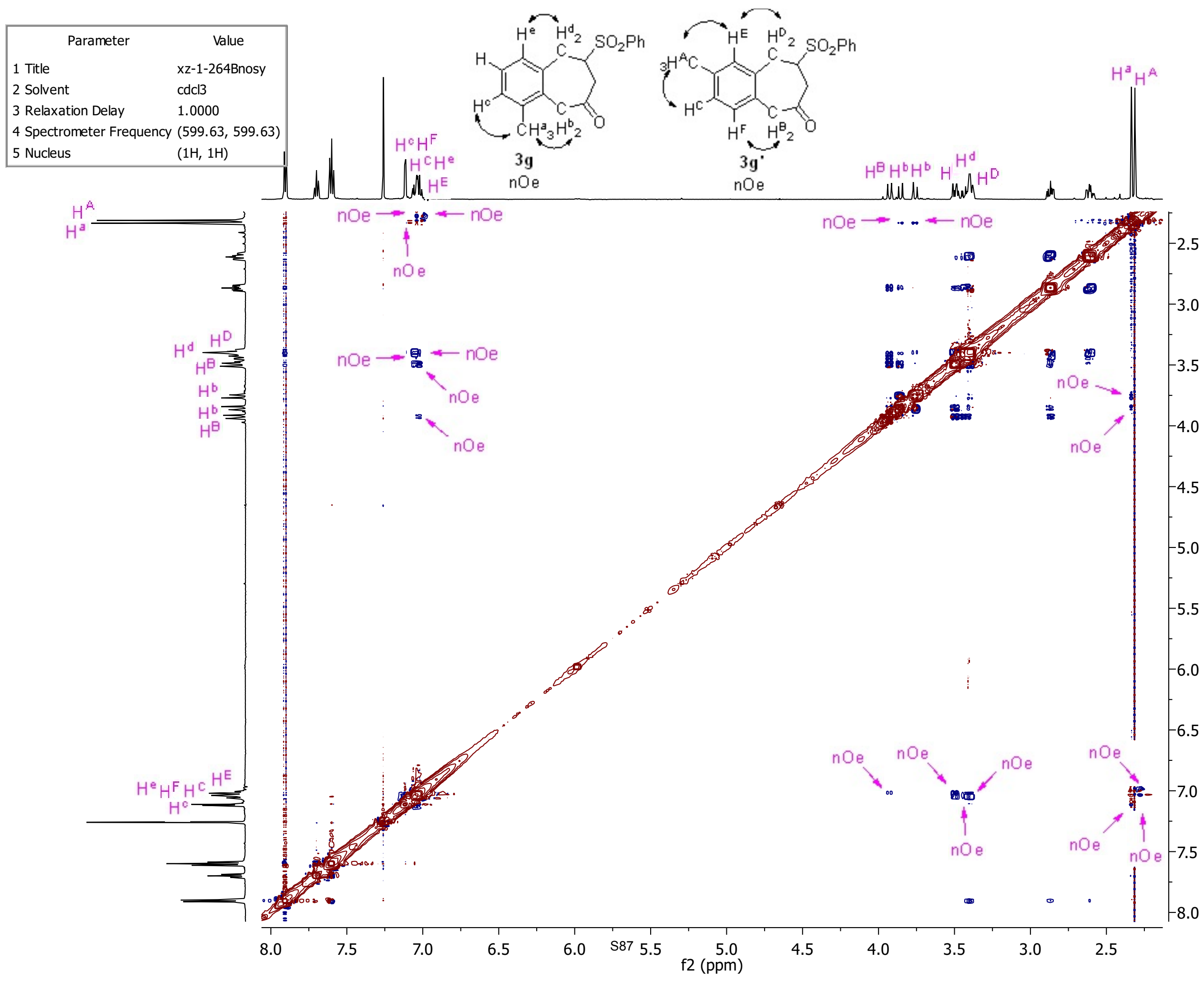

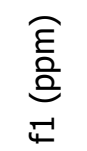




\begin{tabular}{|c|c|}
\hline Parameter & Value \\
\hline 1 Title & xz-1-264Btotal-500-Cbiaopu \\
\hline 2 Solvent & $\mathrm{CDCl} 3$ \\
\hline 3 Spectrometer Frequency & 125.70 \\
\hline 4 Nucleus & $13 C$ \\
\hline
\end{tabular}

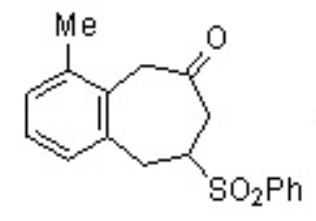

$3 g$

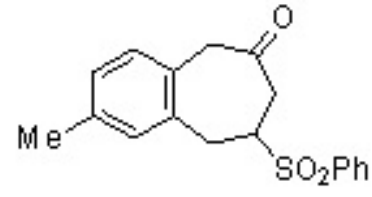

$3 g^{\prime}$

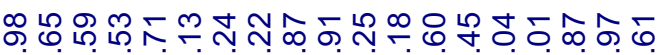

m.

\section{ผุำ}

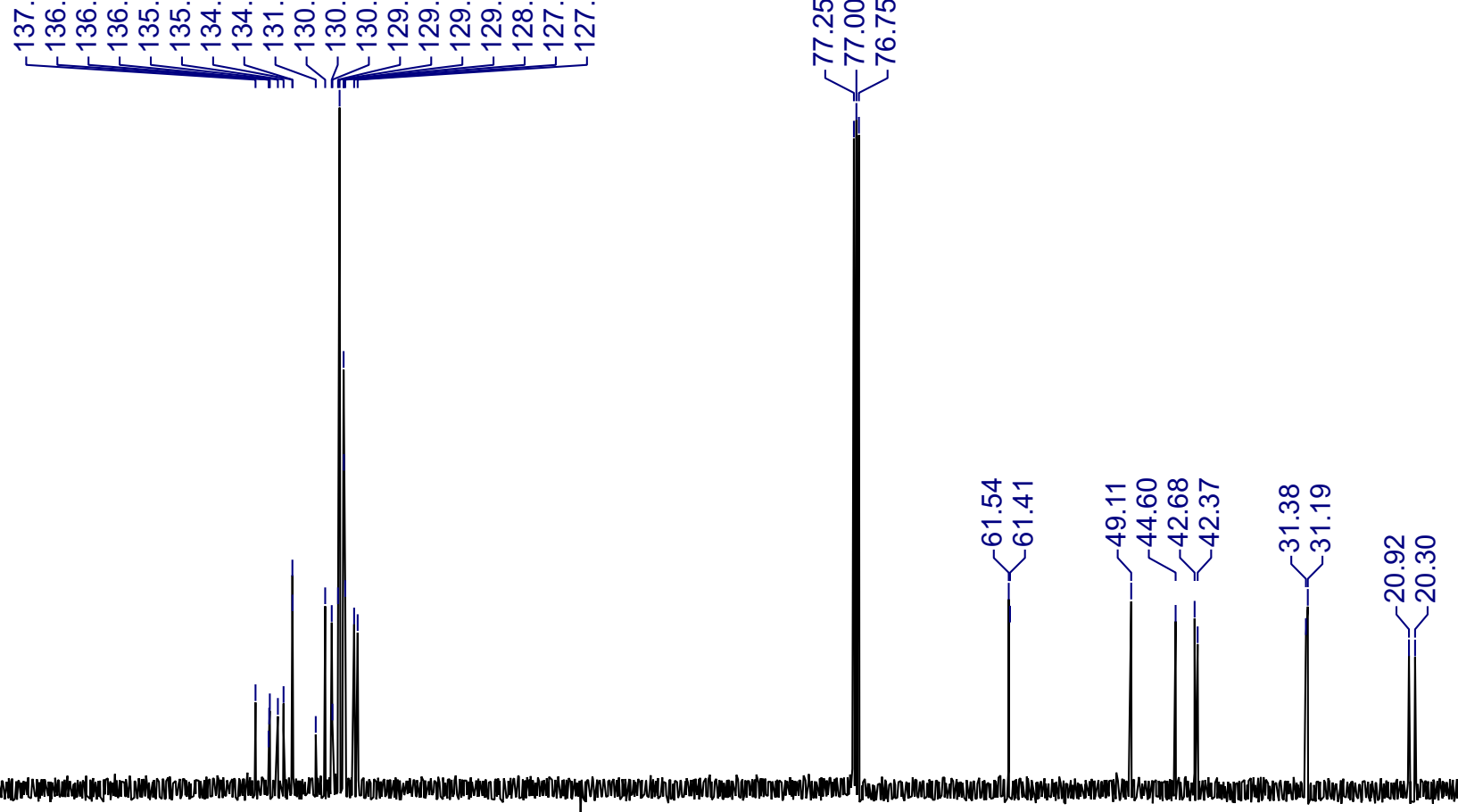

Fô.

กัญิ

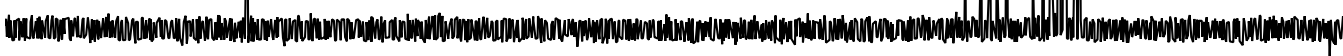

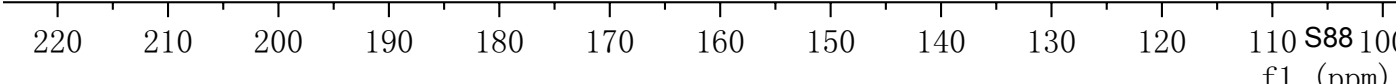

80

70

60

50

40

30

20

10 


\begin{tabular}{|c|c|}
\hline Parameter & Value \\
\hline 1 Title & xz-1-214CnewHbiaopu \\
\hline 2 Solvent & $\mathrm{CDCl} 3$ \\
\hline 3 Spectrometer Frequency & 499.86 \\
\hline 4 Nucleus & $1 \mathrm{H}$ \\
\hline
\end{tabular}
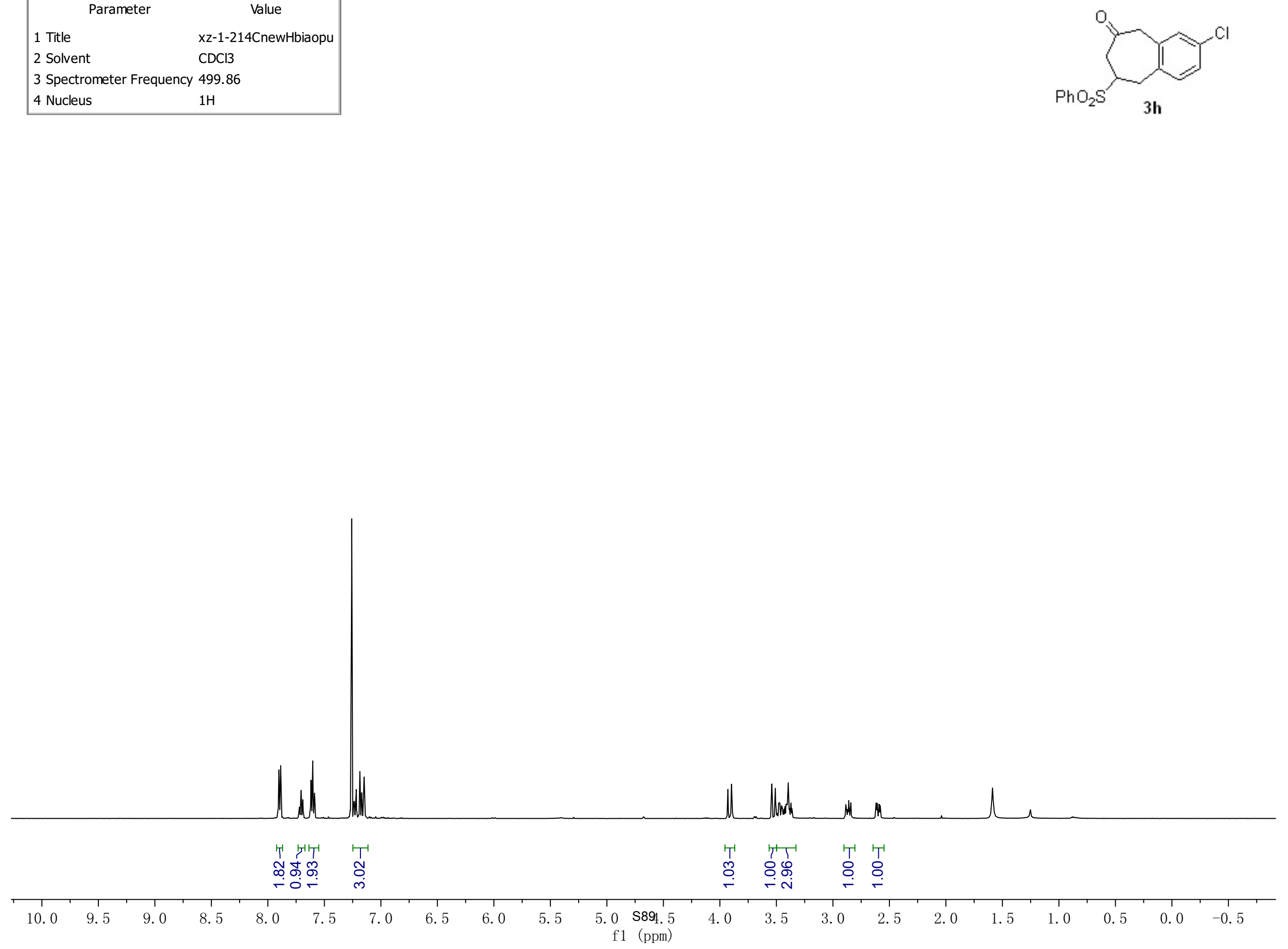


\begin{tabular}{|ll|}
\hline \multicolumn{1}{|c|}{ Parameter } & \multicolumn{1}{c|}{ Value } \\
1 Title & $\mathrm{xz}-1-214$ Cnew500 \\
2 Solvent & $\mathrm{CDCl} 3$ \\
3 Spectrometer Frequency & 125.70 \\
4 Nucleus & $13 \mathrm{C}$ \\
\hline
\end{tabular}
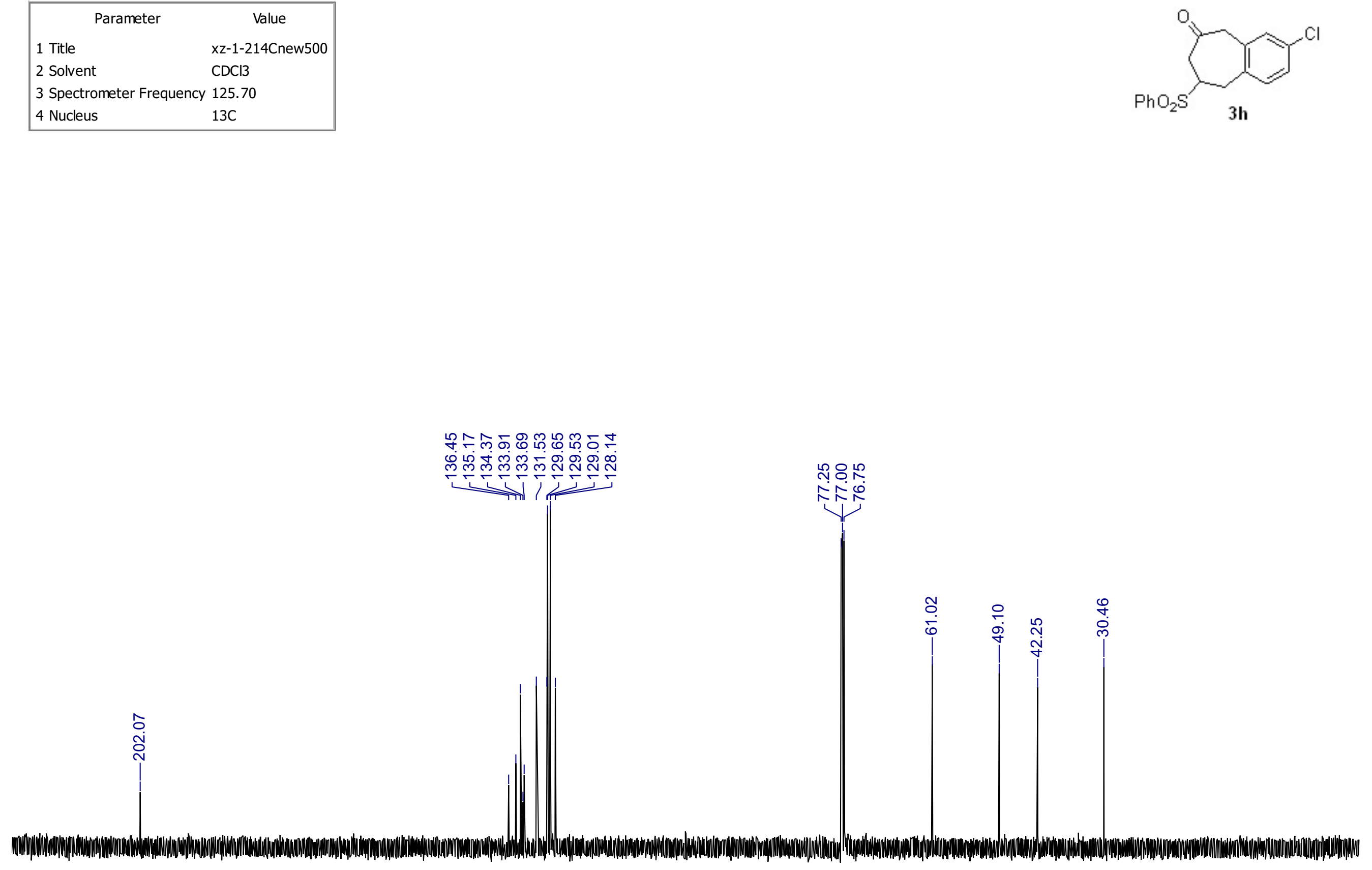

\begin{tabular}{|c|c|c|c|c|c|c|c|c|c|c|c|c|c|c|c|c|c|c|c|c|c|c|}
\hline $\begin{array}{c}1 \\
220\end{array}$ & 210 & 200 & $\begin{array}{c}1 \\
190\end{array}$ & $\begin{array}{c}1 \\
180\end{array}$ & $\begin{array}{c}1 \\
170\end{array}$ & $\begin{array}{c}1 \\
160\end{array}$ & $\begin{array}{c}1 \\
150\end{array}$ & $\begin{array}{c}1 \\
140\end{array}$ & $\begin{array}{c}13 \\
130\end{array}$ & 12 & $\begin{array}{l}110 \text { S90 } 100 \\
\text { f1 (ppm) }\end{array}$ & 90 & 80 & $\begin{array}{l}1 \\
70\end{array}$ & 60 & $\begin{array}{l}1 \\
50\end{array}$ & 40 & 30 & 20 & 10 & 1 & $\begin{array}{c}1 \\
-10\end{array}$ \\
\hline
\end{tabular}




\begin{tabular}{|ll|}
\hline \multicolumn{2}{|c|}{ Parameter } \\
1 Title & \multicolumn{1}{c|}{ Value } \\
2 Solvent & $\mathrm{CDCl} 3$ \\
3 Spectrometer Frequency & 499.86 \\
4 Nucleus & $1 \mathrm{H}$ \\
\hline
\end{tabular}
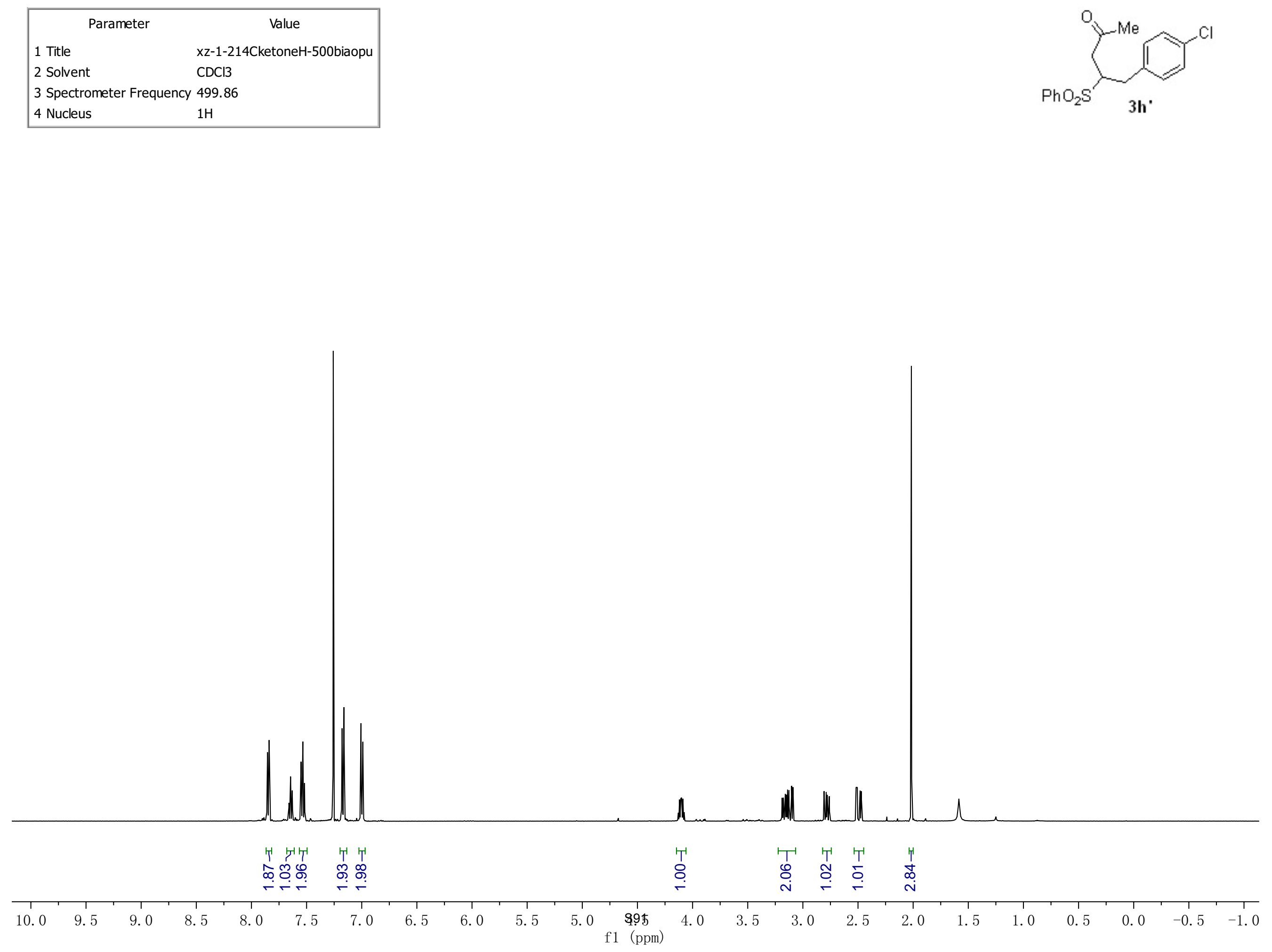


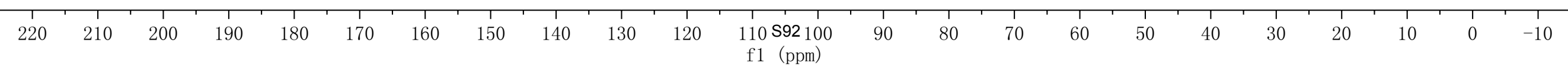




\begin{tabular}{|ll|}
\hline \multicolumn{1}{|c|}{ Parameter } & \multicolumn{1}{c|}{ Value } \\
1 Title & $\mathrm{xz}-1-255 \mathrm{H} 500$ \\
2 Solvent & $\mathrm{CDCl} 3$ \\
3 Spectrometer Frequency & 499.86 \\
4 Nucleus & $1 \mathrm{H}$ \\
\hline
\end{tabular}
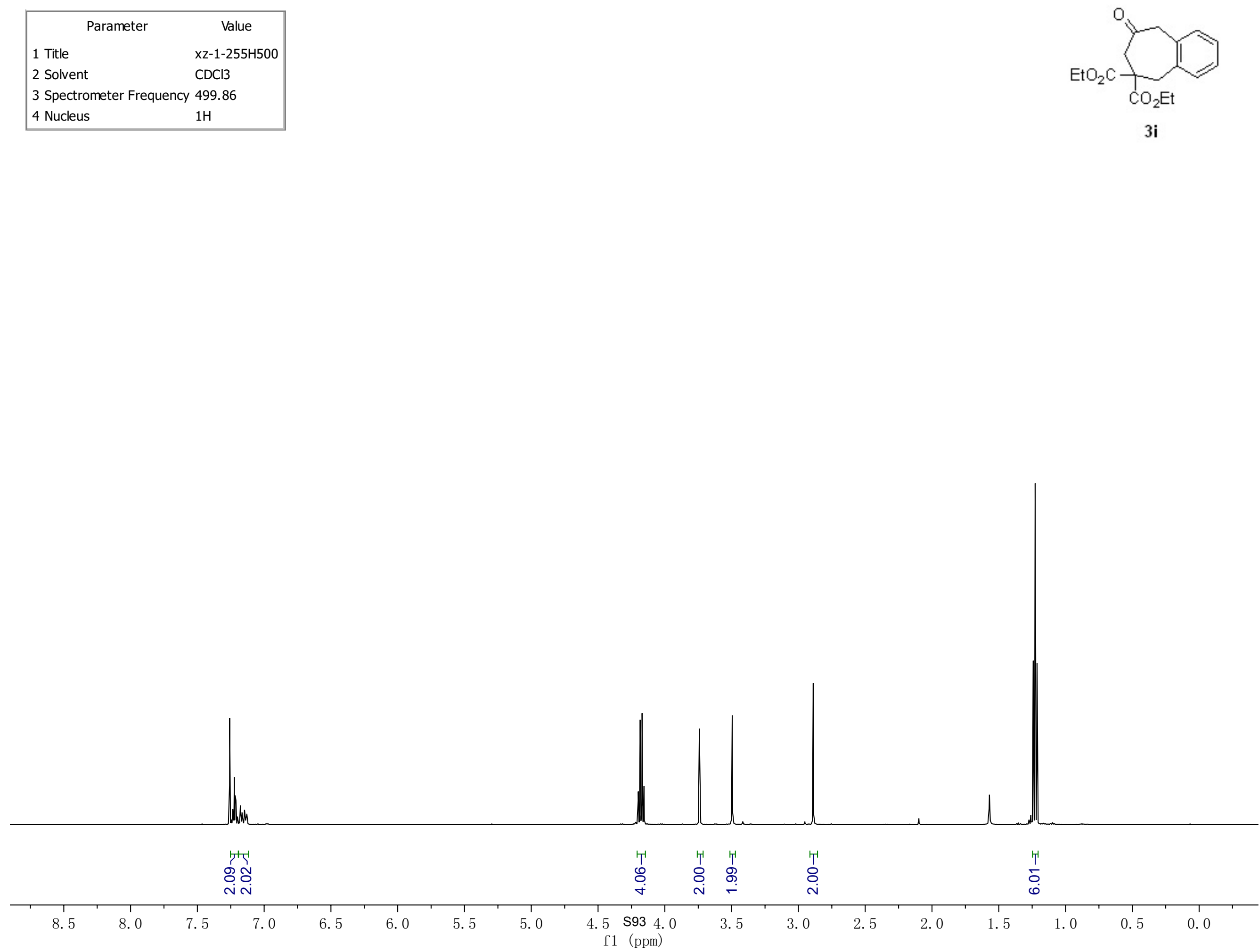


\begin{tabular}{|ll|}
\hline \multicolumn{1}{|c|}{ Parameter } & \multicolumn{1}{c|}{ Value } \\
1 Title & $\mathrm{xz}-1-255 \mathrm{AC} 500$ \\
2 Solvent & $\mathrm{CDCl} 3$ \\
3 Spectrometer Frequency & 125.70 \\
4 Nucleus & $13 \mathrm{C}$ \\
\hline
\end{tabular}

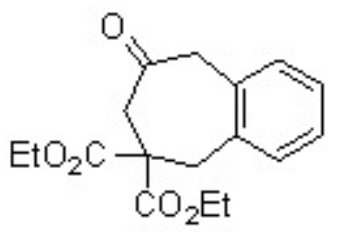

$3 \mathbf{i}$

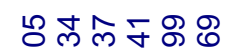

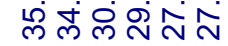

8.

เิ

ণั่
กิ

กิ

|

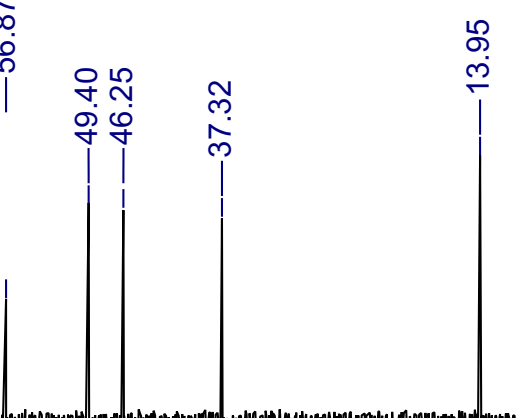




\begin{tabular}{|ll|}
\hline \multicolumn{1}{|c|}{ Parameter } & \multicolumn{1}{c|}{ Value } \\
1 Title & $\mathrm{xz}-1-266 \mathrm{BH} 500$ \\
2 Solvent & $\mathrm{CDCl} 3$ \\
3 Spectrometer Frequency & 499.86 \\
4 Nucleus & $1 \mathrm{H}$ \\
\hline
\end{tabular}

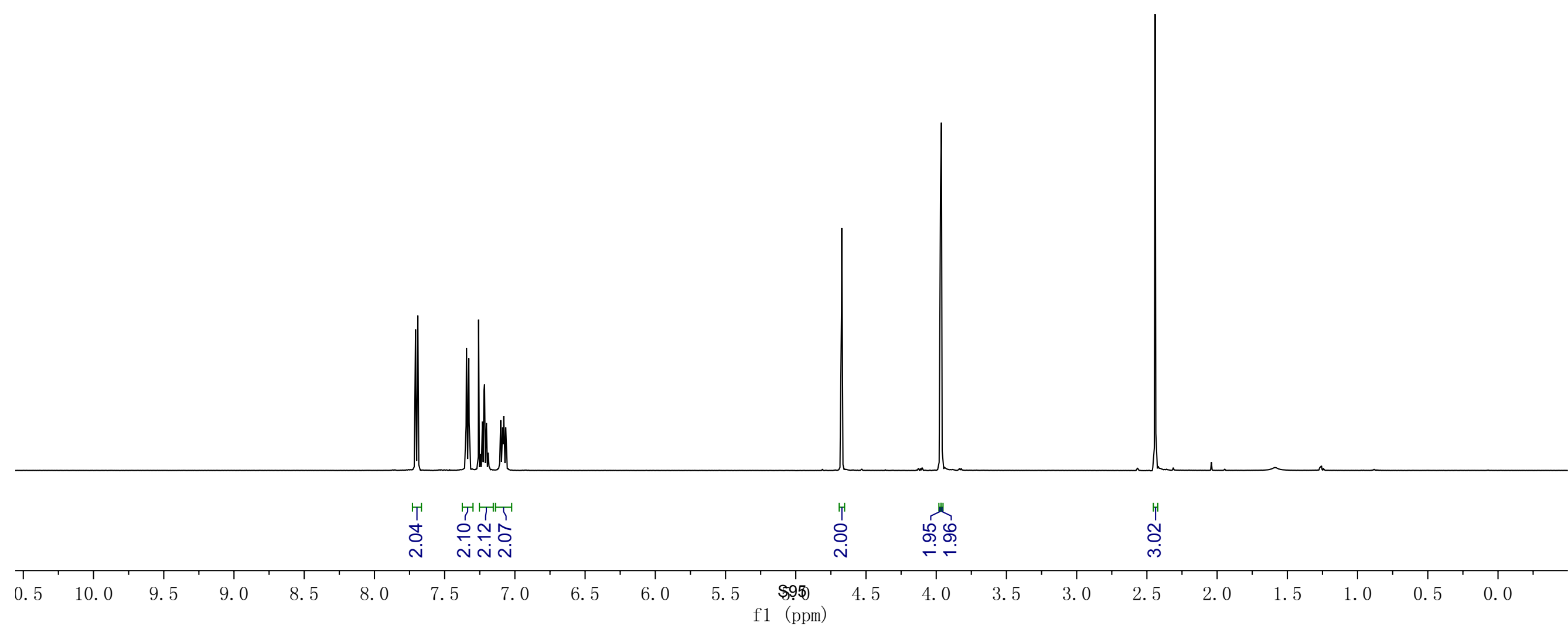




\begin{tabular}{|ll|}
\hline \multicolumn{1}{|c|}{ Parameter } & \multicolumn{1}{c|}{ Value } \\
1 Title & $\mathrm{xz}-1-266 \mathrm{BC} 500$ \\
2 Solvent & $\mathrm{CDCl} 3$ \\
3 Spectrometer Frequency & 125.70 \\
4 Nucleus & $13 \mathrm{C}$ \\
\hline
\end{tabular}

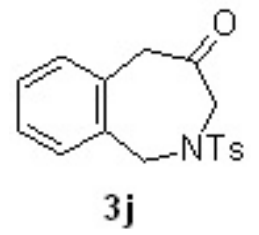

응드융ㅇㅇㅇㅇ

फु m̄ं

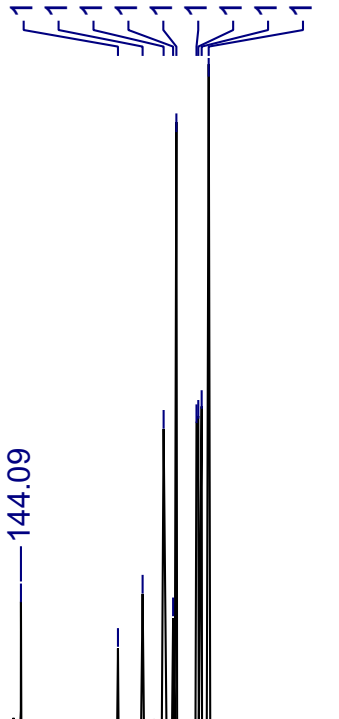

กุำ

NAo

败 经品

เิ่ กิ่

iी 10

莡

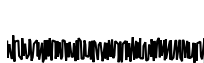
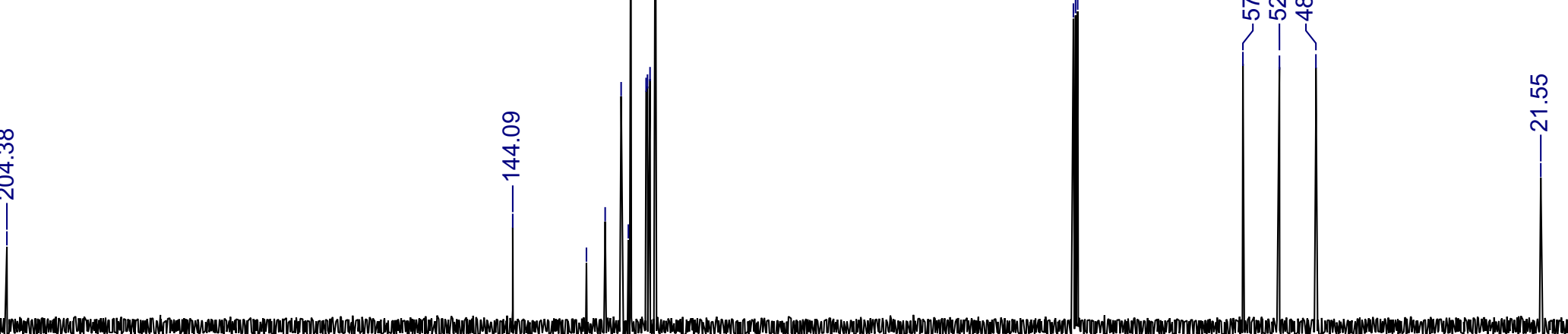

$\stackrel{\sim}{\stackrel{\sim}{\sim}}$

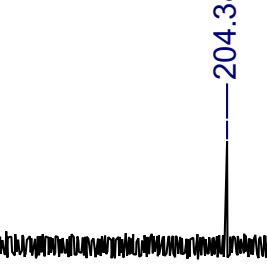

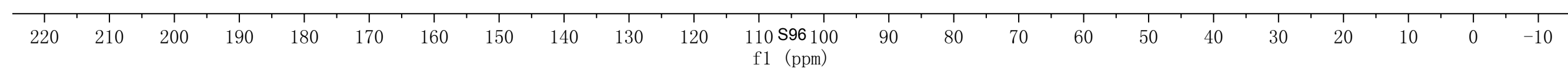




\begin{tabular}{|ll|}
\hline \multicolumn{1}{|c|}{ Parameter } & \multicolumn{1}{c|}{ Value } \\
1 Title & $\mathrm{xz}-1-202$ BtotalH \\
2 Solvent & $\mathrm{CDCl3}$ \\
3 Spectrometer Frequency & 499.86 \\
4 Nucleus & $1 \mathrm{H}$ \\
\hline
\end{tabular}

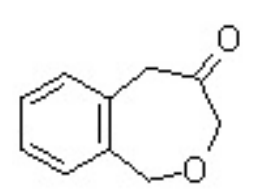

$3 k$

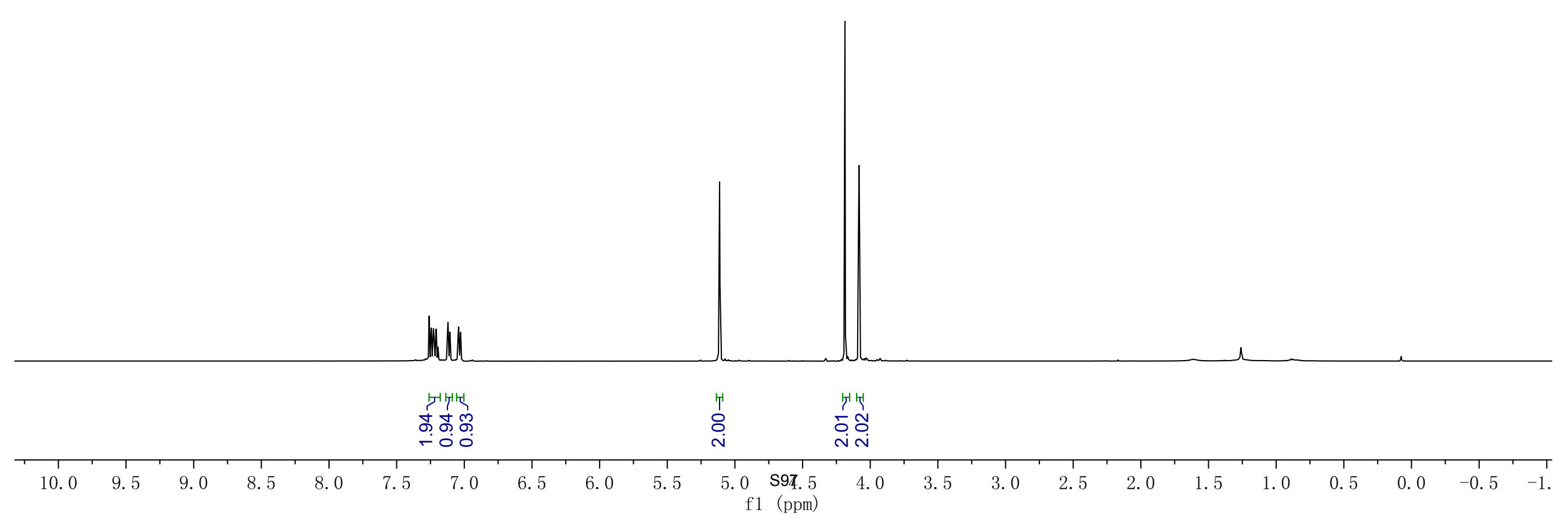




\begin{tabular}{|ll|}
\hline \multicolumn{2}{|c|}{ Parameter } \\
1 Title & $\mathrm{xz}-1-202 \mathrm{BCbiaopu} 500$ \\
2 Solvent & $\mathrm{CDCl} 3$ \\
3 Spectrometer Frequency & 125.70 \\
4 Nucleus & $13 \mathrm{C}$ \\
\hline
\end{tabular}
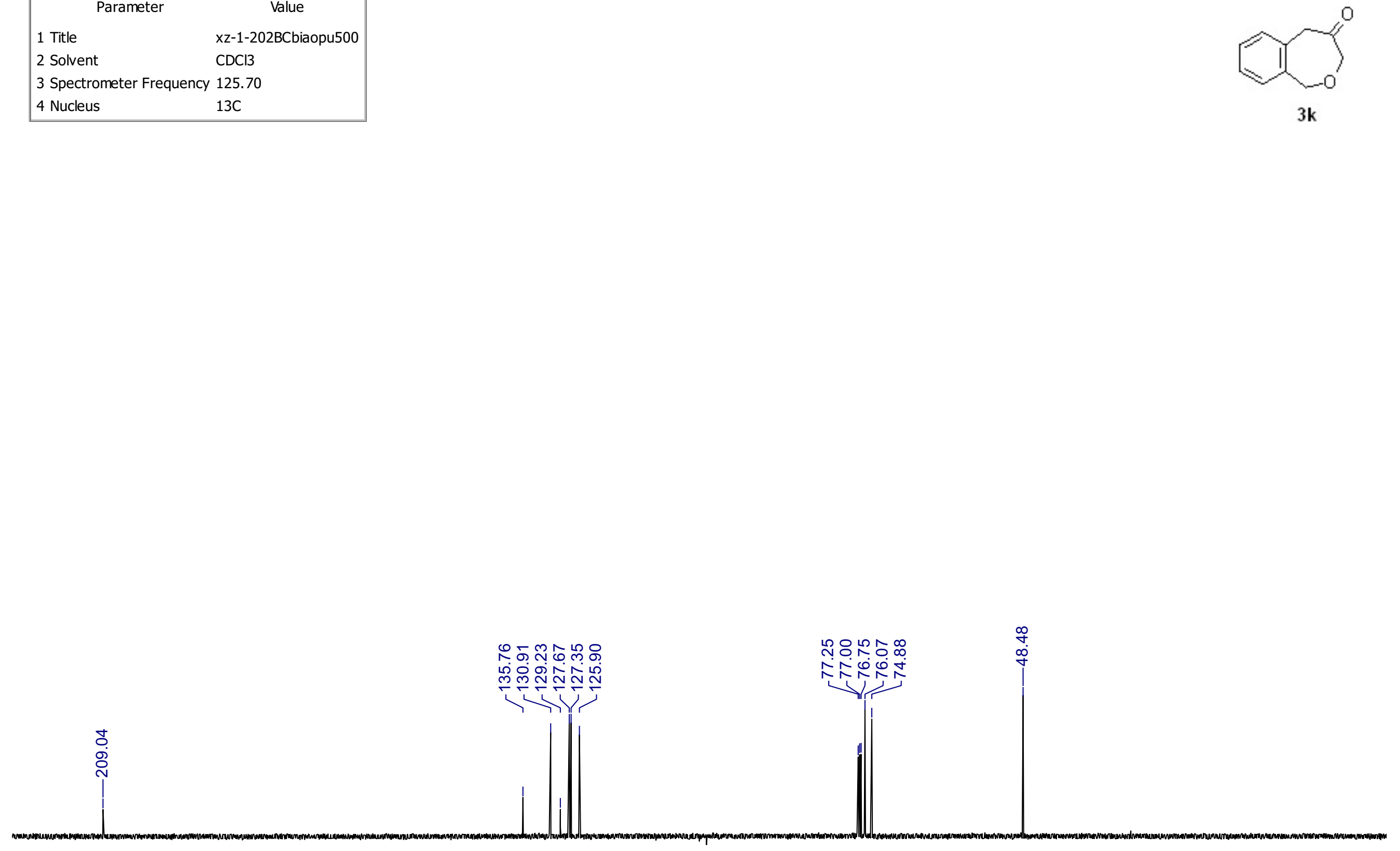

\begin{tabular}{|c|c|c|c|c|c|c|c|c|c|c|c|c|c|c|c|c|c|c|c|c|c|c|}
\hline 220 & 210 & 200 & 190 & 180 & 170 & 160 & 150 & 140 & 130 & 120 & $\begin{array}{c}110 S 98100 \\
\mathrm{f} 1 \quad(\mathrm{ppm})\end{array}$ & 90 & 80 & 70 & 60 & 50 & 40 & 30 & 20 & 10 & 0 & -10 \\
\hline
\end{tabular}




\begin{tabular}{|ll|}
\hline \multicolumn{1}{|c|}{ Parameter } & \multicolumn{1}{c|}{ Value } \\
1 Title & chy-3-2-84-P-1H \\
2 Solvent & CDCl3 \\
3 Spectrometer Frequency & 499.86 \\
4 Nucleus & $1 \mathrm{H}$ \\
\hline
\end{tabular}
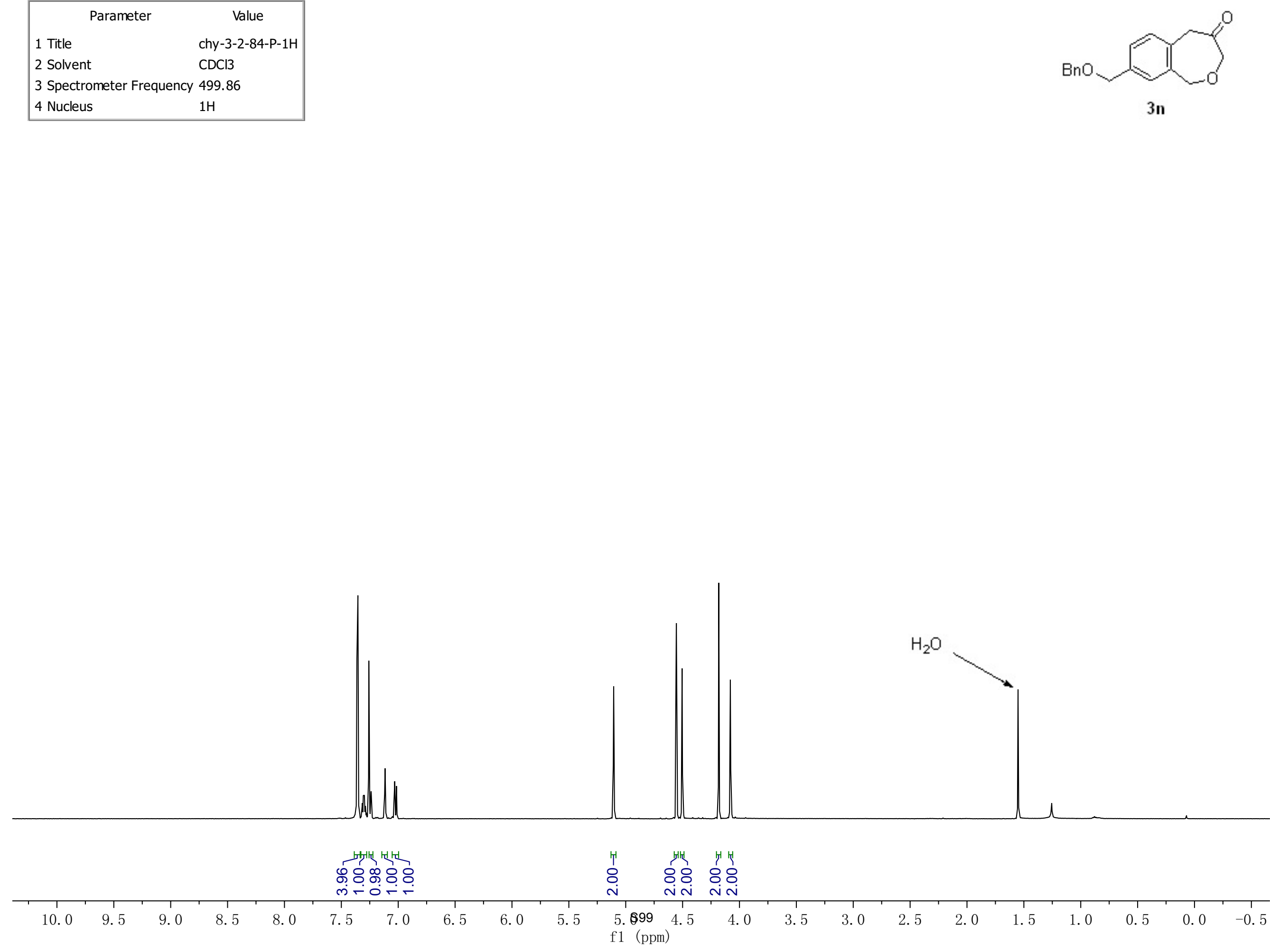


\begin{tabular}{|ll|}
\hline \multicolumn{1}{|c|}{ Parameter } & \multicolumn{1}{c|}{ Value } \\
1 Title & chy-3-2-84-P-13C \\
2 Solvent & CDCl3 \\
3 Spectrometer Frequency & 125.70 \\
4 Nucleus & $13 \mathrm{C}$ \\
\hline
\end{tabular}
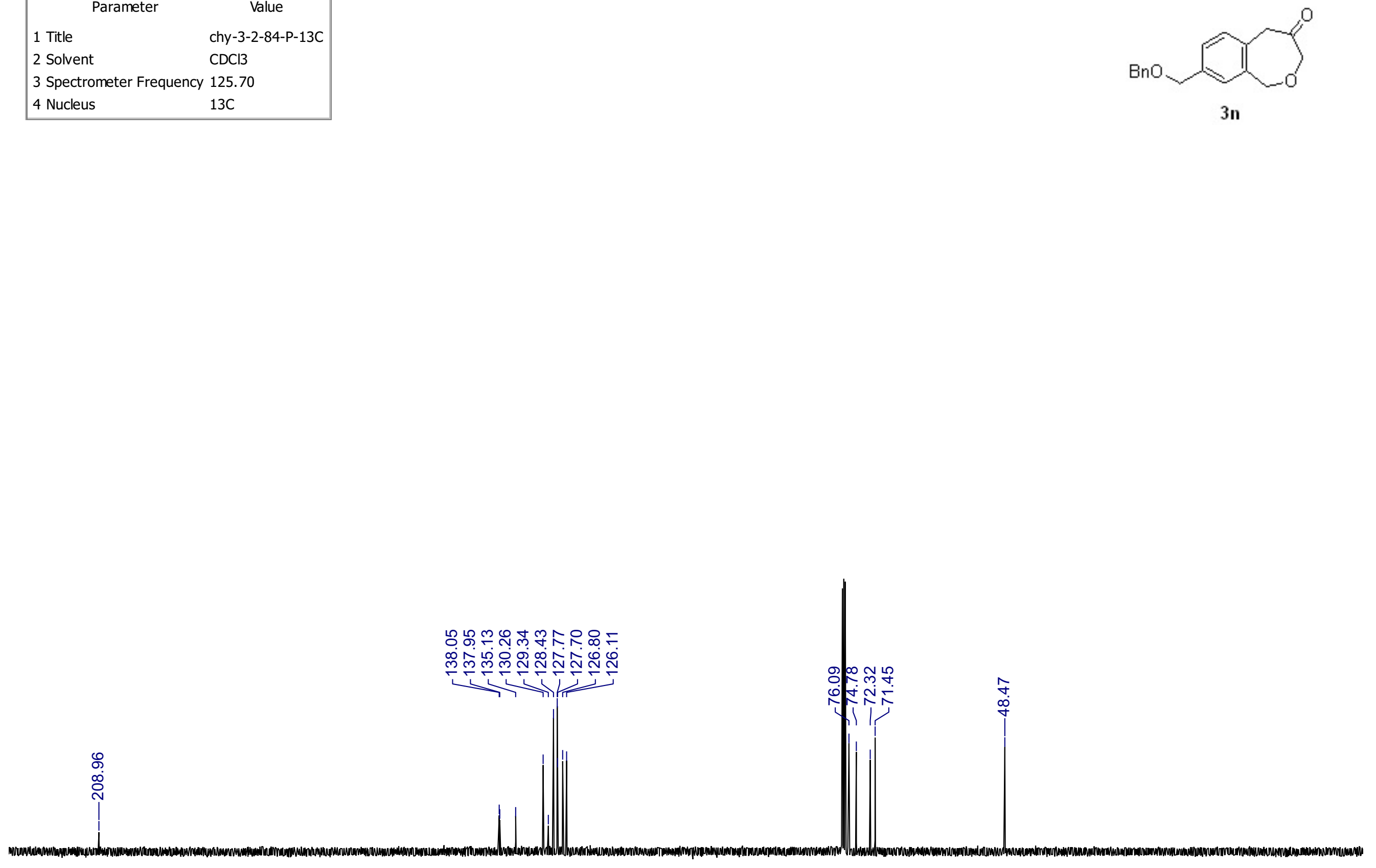

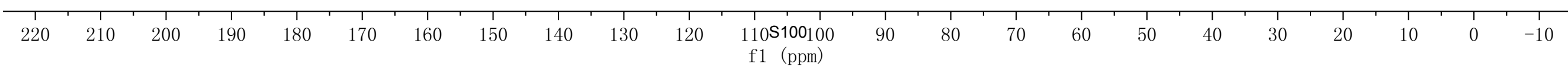




\begin{tabular}{|ll|}
\hline \multicolumn{1}{|c|}{ Parameter } & \multicolumn{1}{c|}{ Value } \\
1 Title & chy-3-106-2-P-1H \\
2 Solvent & $\mathrm{CDCl} 3$ \\
3 Spectrometer Frequency & 499.86 \\
4 Nucleus & $1 \mathrm{H}$ \\
\hline
\end{tabular}
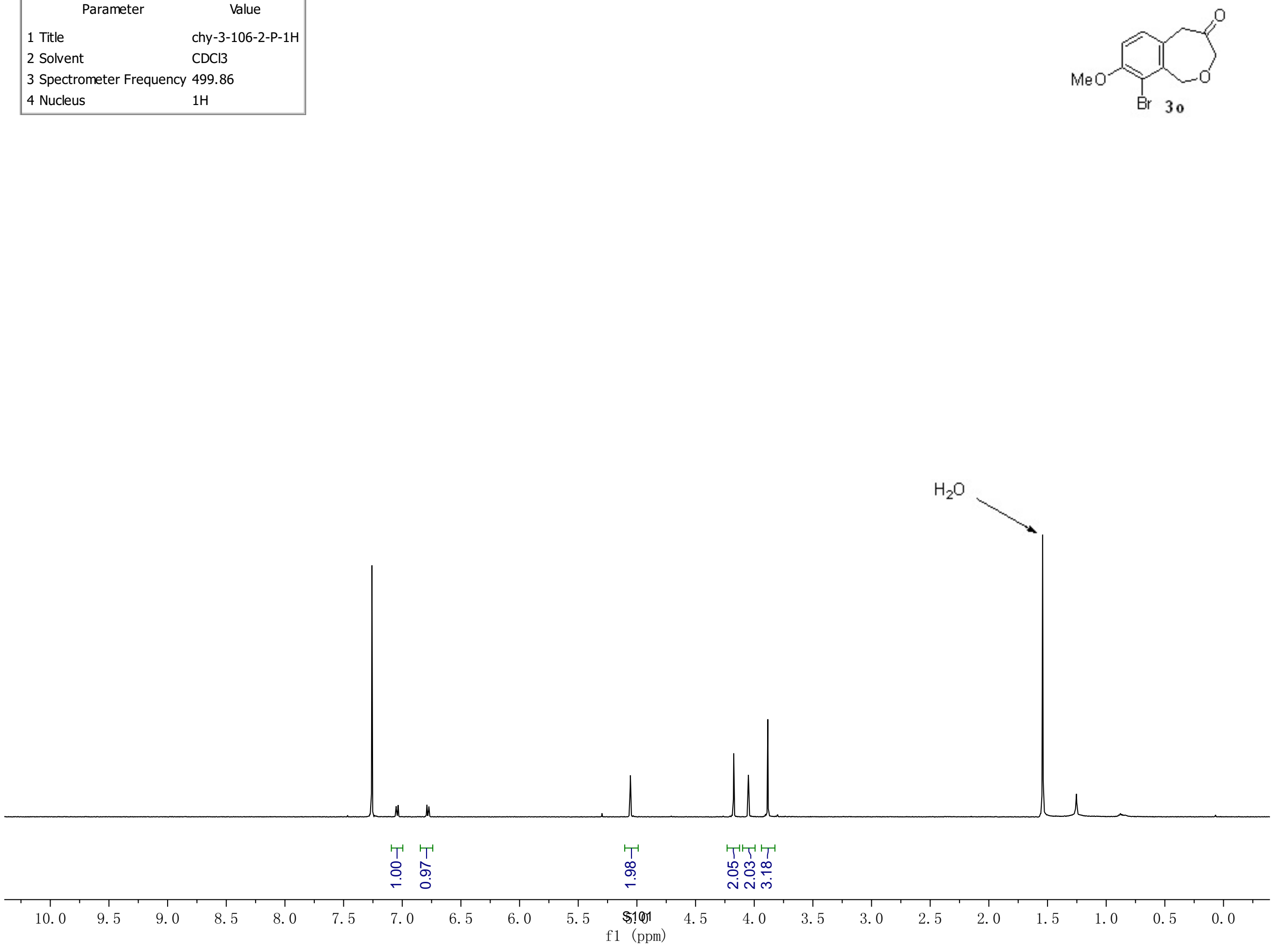


\begin{tabular}{|ll|}
\hline \multicolumn{1}{|c|}{ Parameter } & \multicolumn{1}{c|}{ Value } \\
1 Title & chy-3-106-2-P-13C \\
2 Solvent & CDCl3 \\
3 Spectrometer Frequency & 125.70 \\
4 Nucleus & $13 \mathrm{C}$ \\
\hline
\end{tabular}
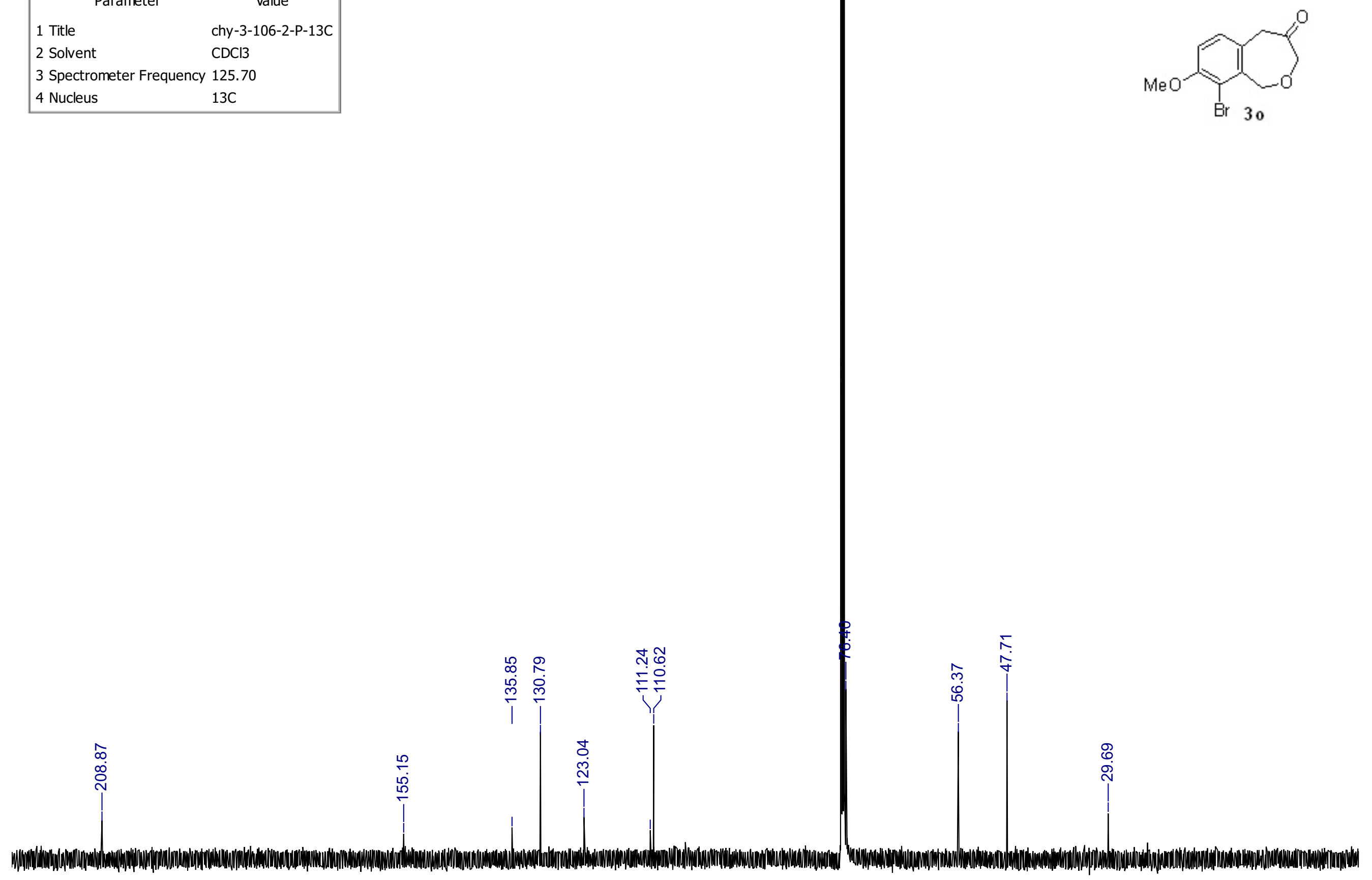

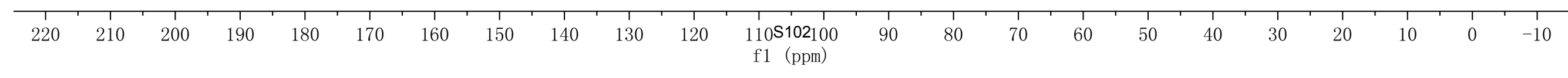




\begin{tabular}{|ll|}
\hline \multicolumn{1}{|c|}{ Parameter } & \multicolumn{1}{c|}{ Value } \\
1 Title & $\mathrm{xz}-1-228 \mathrm{BH} 500 \mathrm{biaopu}$ \\
2 Solvent & $\mathrm{CDCl} 3$ \\
3 Spectrometer Frequency & 499.86 \\
4 Nucleus & $1 \mathrm{H}$ \\
\hline
\end{tabular}

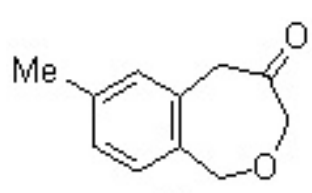

31

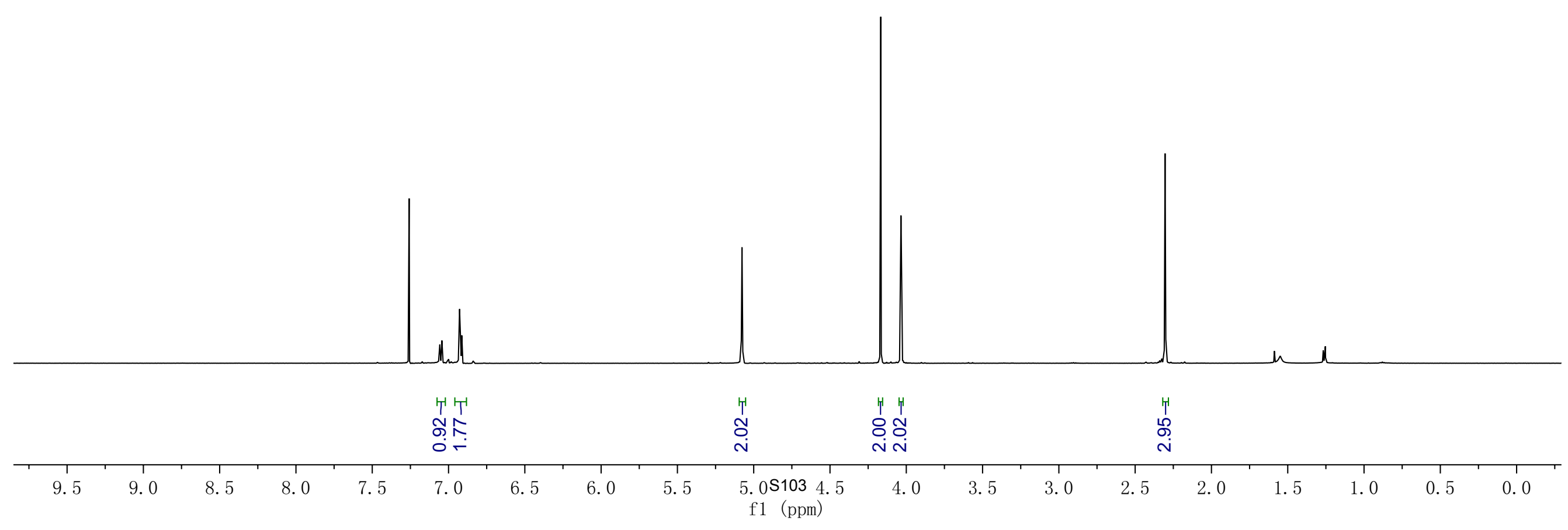




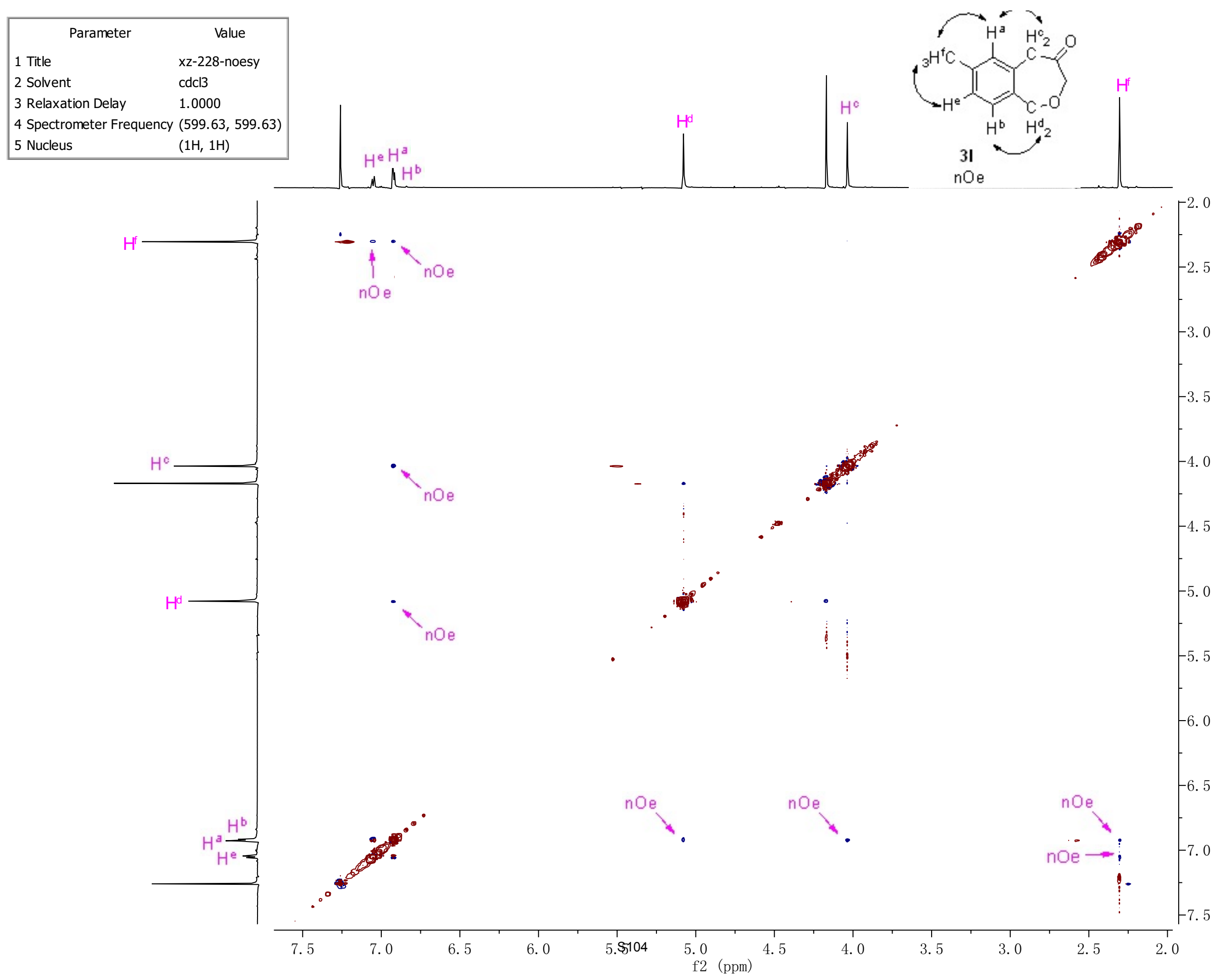




\begin{tabular}{|ll|}
\hline \multicolumn{1}{|c|}{ Parameter } & \multicolumn{1}{c|}{ Value } \\
1 Title & $\mathrm{xz}-1-228 \mathrm{BC500biaopu}$ \\
2 Solvent & $\mathrm{CDCl} 3$ \\
3 Spectrometer Frequency & 125.70 \\
4 Nucleus & $13 \mathrm{C}$ \\
\hline
\end{tabular}

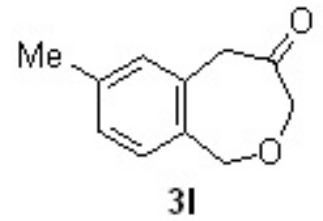

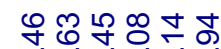

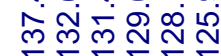

$\stackrel{\Upsilon}{\check{O}}$

ஓ्尺े

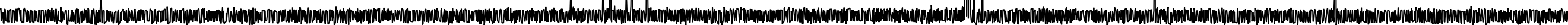

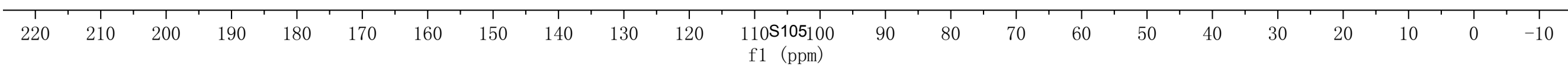




\begin{tabular}{|ll|}
\hline \multicolumn{1}{|c|}{ Parameter } & \multicolumn{1}{c|}{ Value } \\
1 Title & $\mathrm{xz}-1-232 \mathrm{BH} 500 \mathrm{biaopu}$ \\
2 Solvent & $\mathrm{CDCl3}$ \\
3 Relaxation Delay & 16.0000 \\
4 Spectrometer Frequency & 499.86 \\
\hline
\end{tabular}
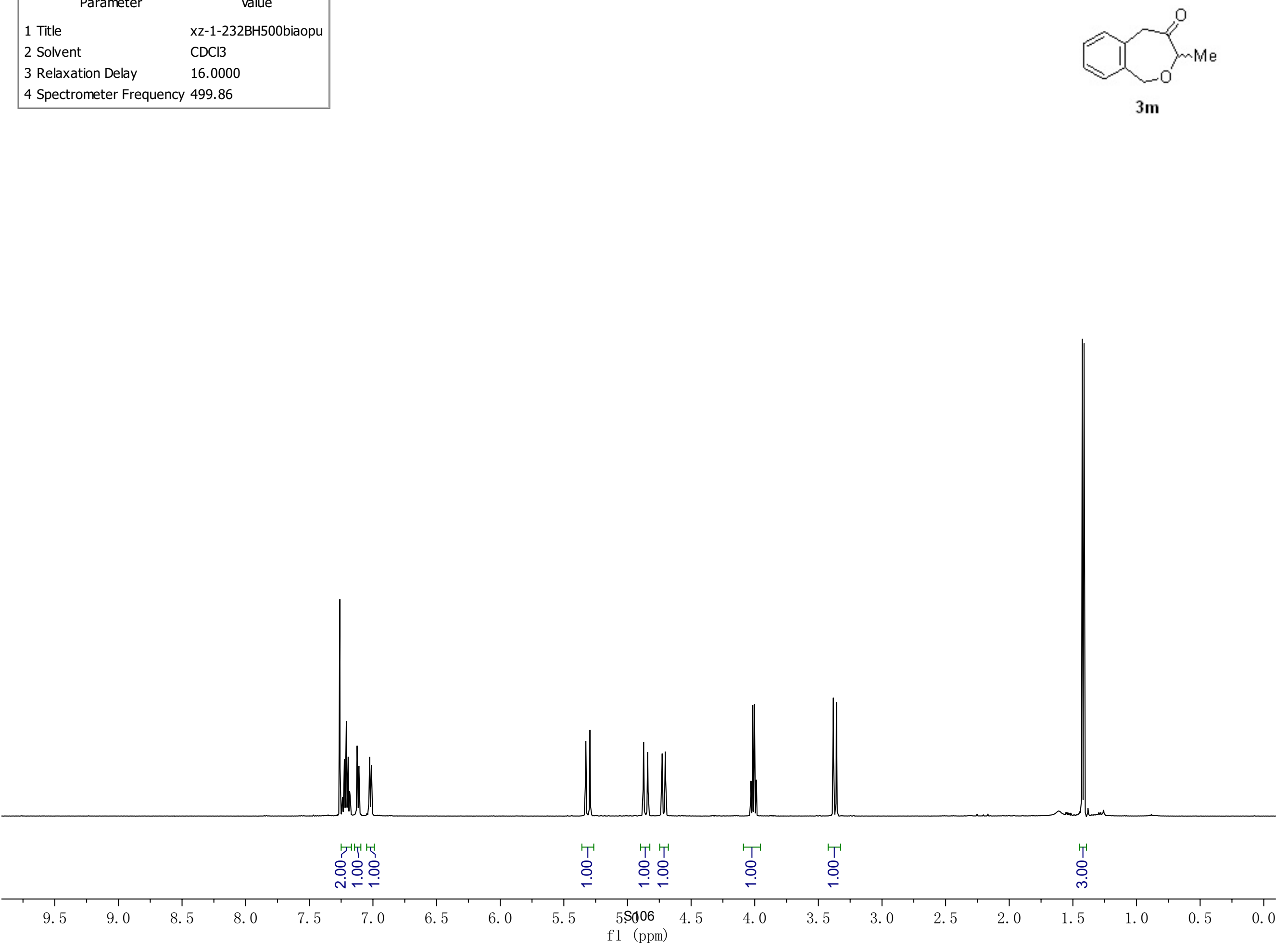


\begin{tabular}{|ll|}
\hline \multicolumn{1}{|c|}{ Parameter } & \multicolumn{1}{c|}{ Value } \\
1 Title & $\mathrm{xz}-1-232 \mathrm{BC} 500$ \\
2 Solvent & $\mathrm{CDCl} 3$ \\
3 Relaxation Delay & 1.0000 \\
4 Spectrometer Frequency & 125.70 \\
\hline
\end{tabular}

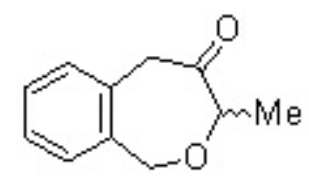

$3 \mathrm{~m}$

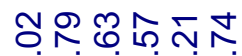

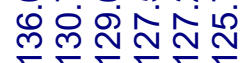

둔

$>$

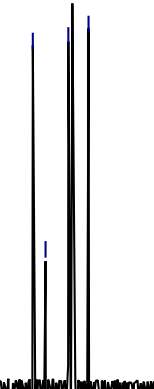

so

\%

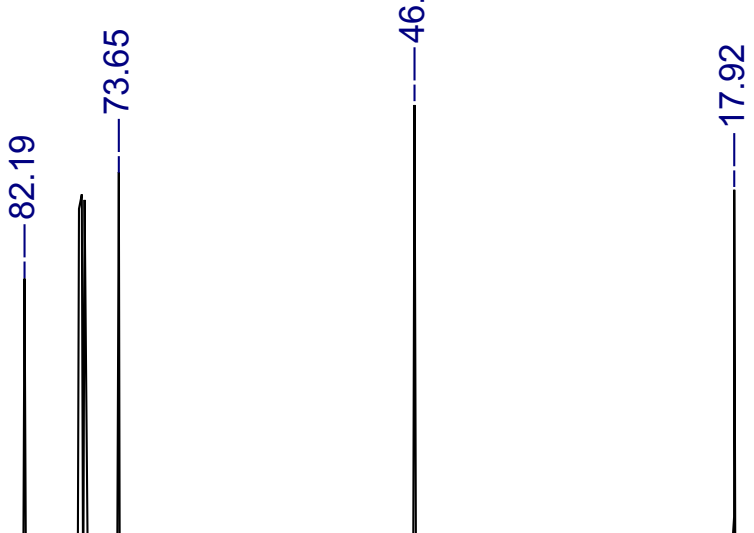

$\stackrel{\stackrel{\sim}{\sim}}{\stackrel{1}{*}}$

s

인

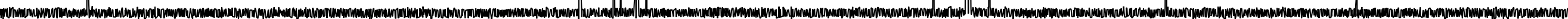

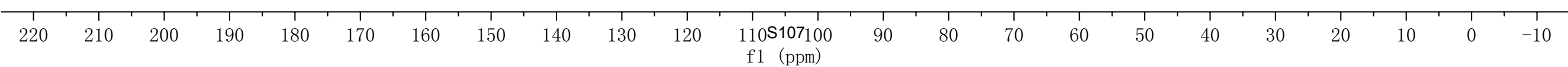




\begin{tabular}{|ll|}
\hline \multicolumn{1}{|c|}{ Parameter } & \multicolumn{1}{c|}{ Value } \\
1 Title & chy-3-74-2-P-1H \\
2 Solvent & $\mathrm{CDCl} 3$ \\
3 Spectrometer Frequency & 499.86 \\
4 Nucleus & $1 \mathrm{H}$ \\
\hline
\end{tabular}
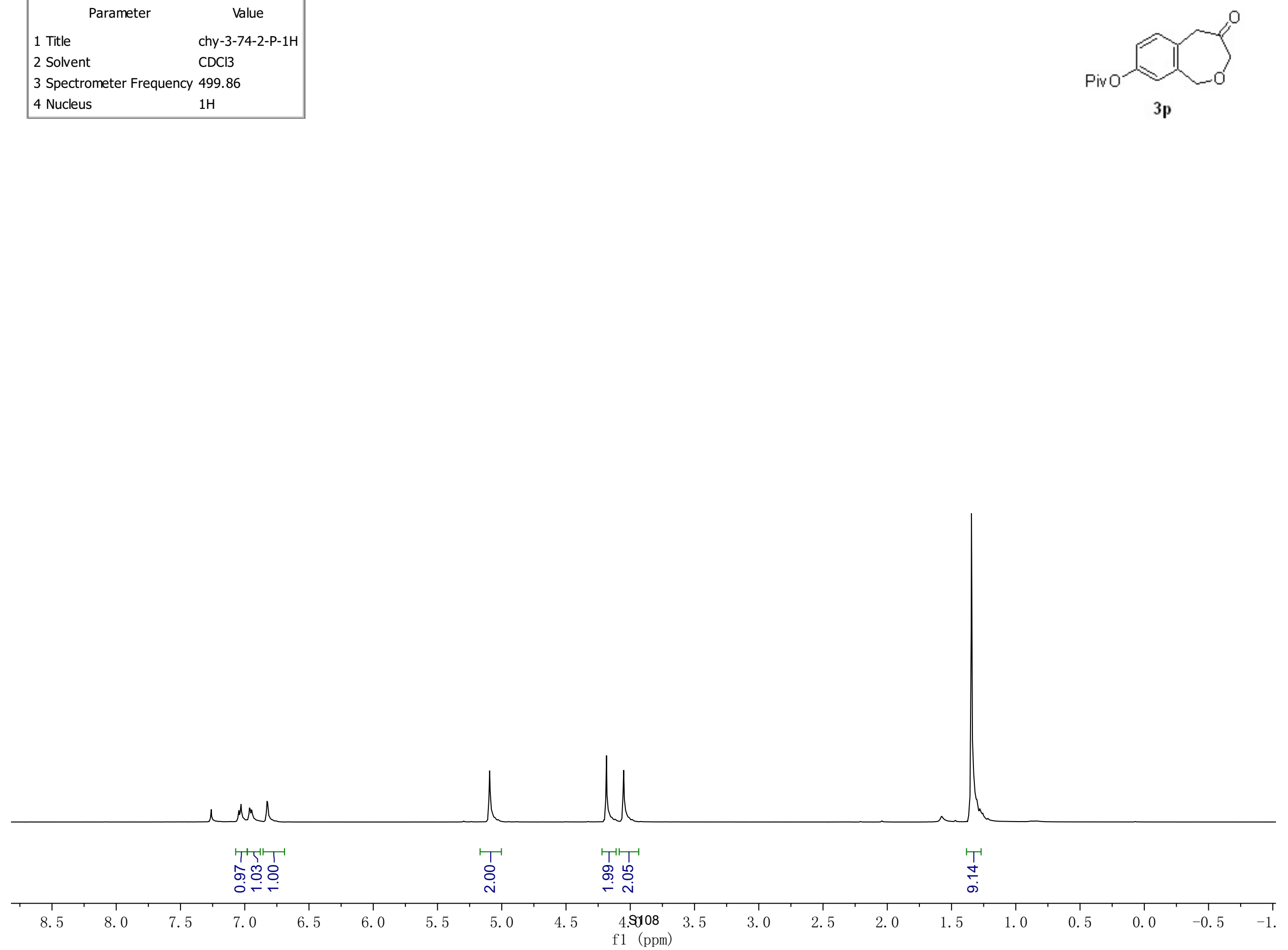


\begin{tabular}{|ll|}
\hline \multicolumn{1}{|c|}{ Parameter } & \multicolumn{1}{c|}{ Value } \\
1 Title & chy-3-74-2-P-13C \\
2 Solvent & $\mathrm{CDCl} 3$ \\
3 Spectrometer Frequency & 125.70 \\
4 Nucleus & $13 \mathrm{C}$ \\
\hline
\end{tabular}
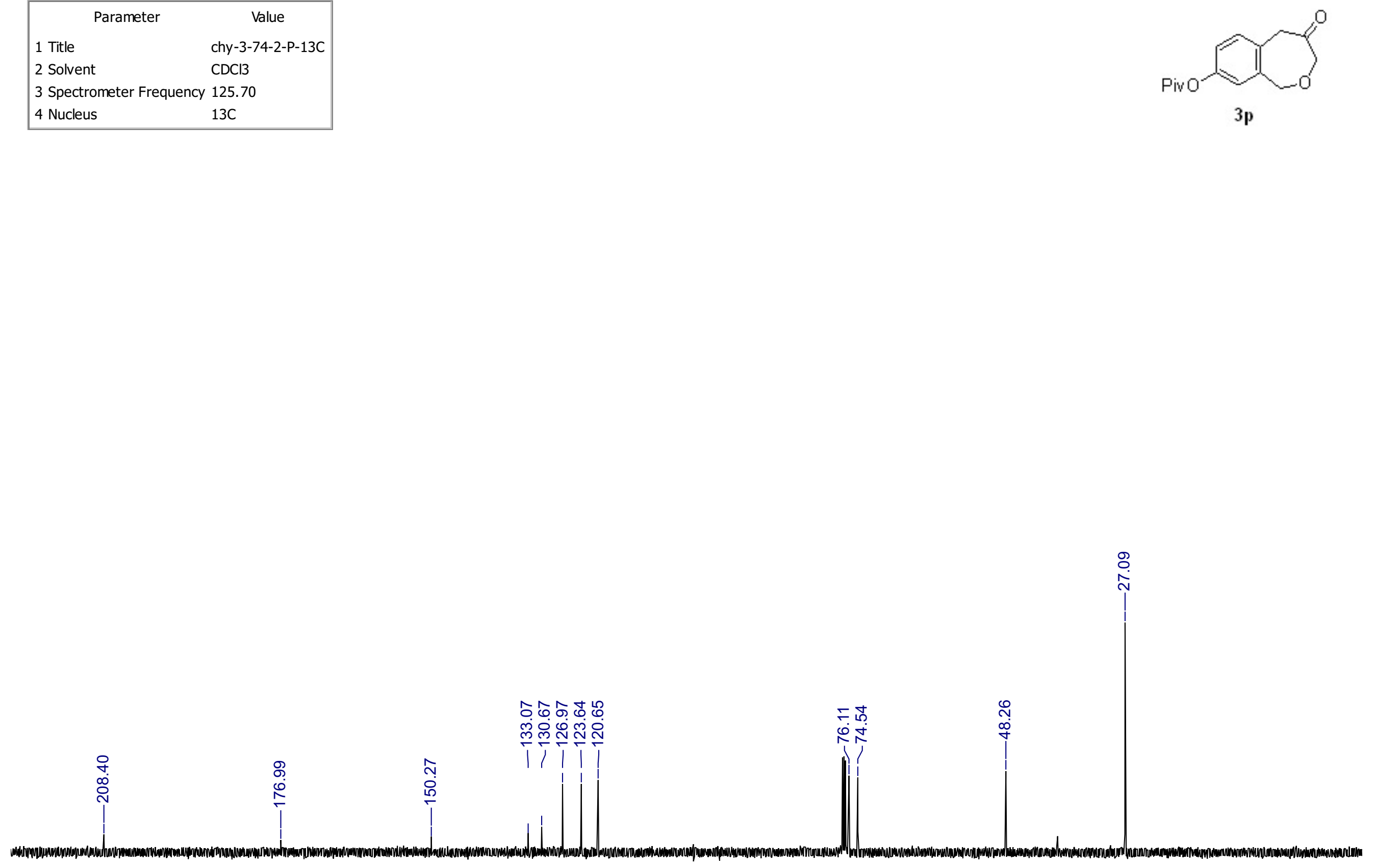

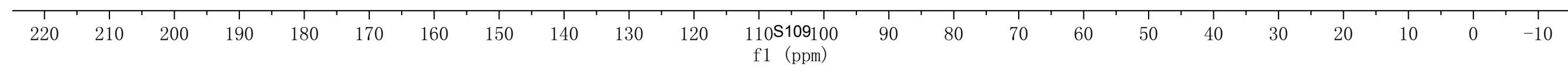




\begin{tabular}{|ll|}
\hline \multicolumn{1}{|c|}{ Parameter } & \multicolumn{1}{c|}{ Value } \\
1 Title & chy-2-211-1-Pro-1H \\
2 Solvent & $\mathrm{CDCl} 3$ \\
3 Spectrometer Frequency & 499.86 \\
4 Nucleus & $1 \mathrm{H}$ \\
\hline
\end{tabular}
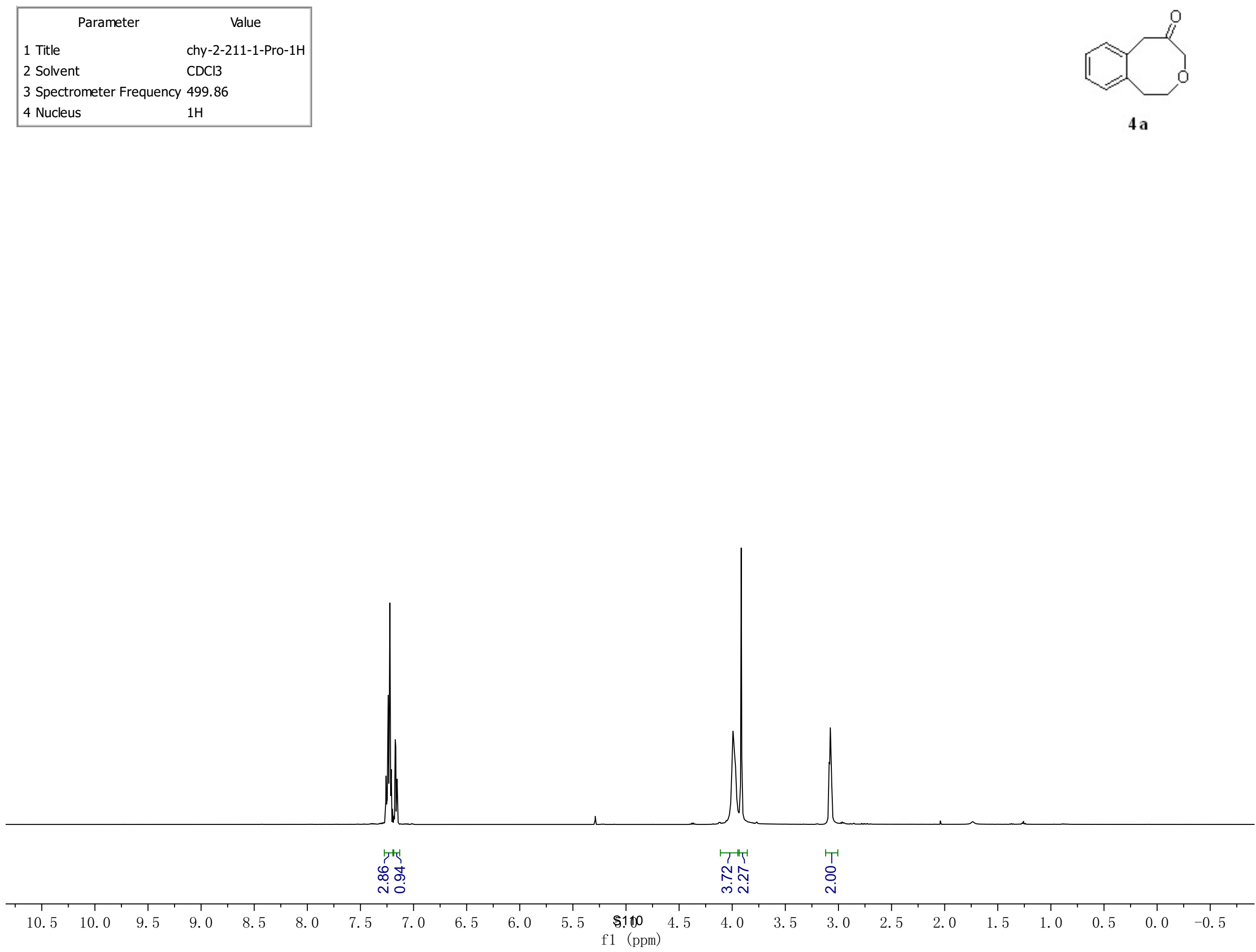


\begin{tabular}{|ll|}
\hline \multicolumn{1}{|c|}{ Parameter } & \multicolumn{1}{c|}{ Value } \\
1 Title & chy-2-211-1-Pro-13C \\
2 Solvent & $\mathrm{CDCl} 3$ \\
3 Spectrometer Frequency & 125.70 \\
4 Nucleus & $13 \mathrm{C}$ \\
\hline
\end{tabular}
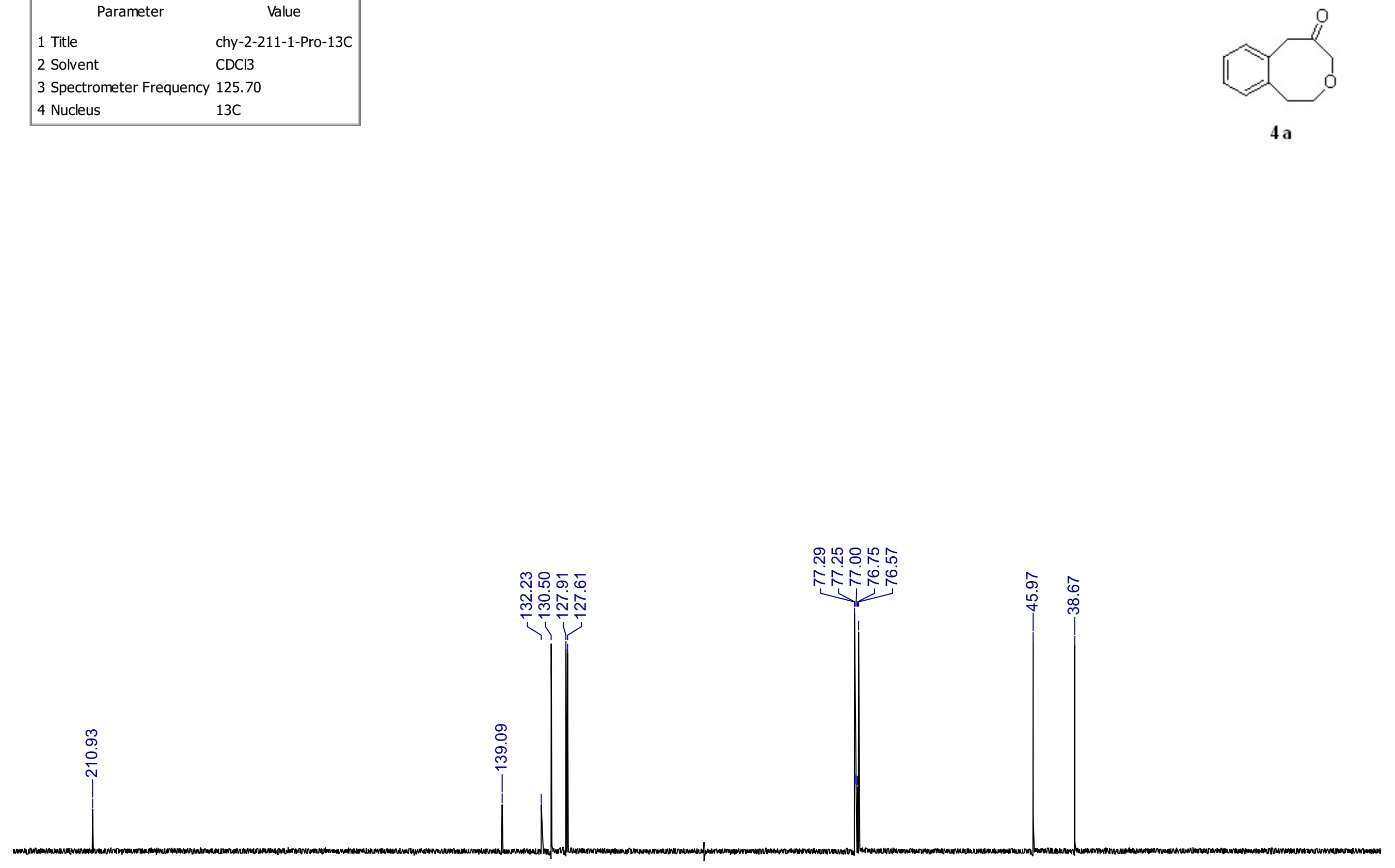

\begin{tabular}{|c|c|c|c|c|c|c|c|c|c|c|c|c|c|c|c|c|c|c|c|c|c|c|}
\hline 220 & 210 & 200 & 190 & $\begin{array}{c}1 \\
180\end{array}$ & 170 & $\begin{array}{c}1 \\
160\end{array}$ & $\begin{array}{c}1 \\
150\end{array}$ & 140 & 130 & 120 & $\begin{array}{l}110 \mathrm{~S} 111100 \\
\mathrm{f} 1 \quad(\mathrm{ppm})\end{array}$ & 90 & 80 & $\begin{array}{l}1 \\
70\end{array}$ & 60 & 50 & 40 & $\begin{array}{l}1 \\
30\end{array}$ & 20 & 10 & $\begin{array}{l}1 \\
0\end{array}$ & -10 \\
\hline
\end{tabular}


3 Spectrometer Frequency 499.86

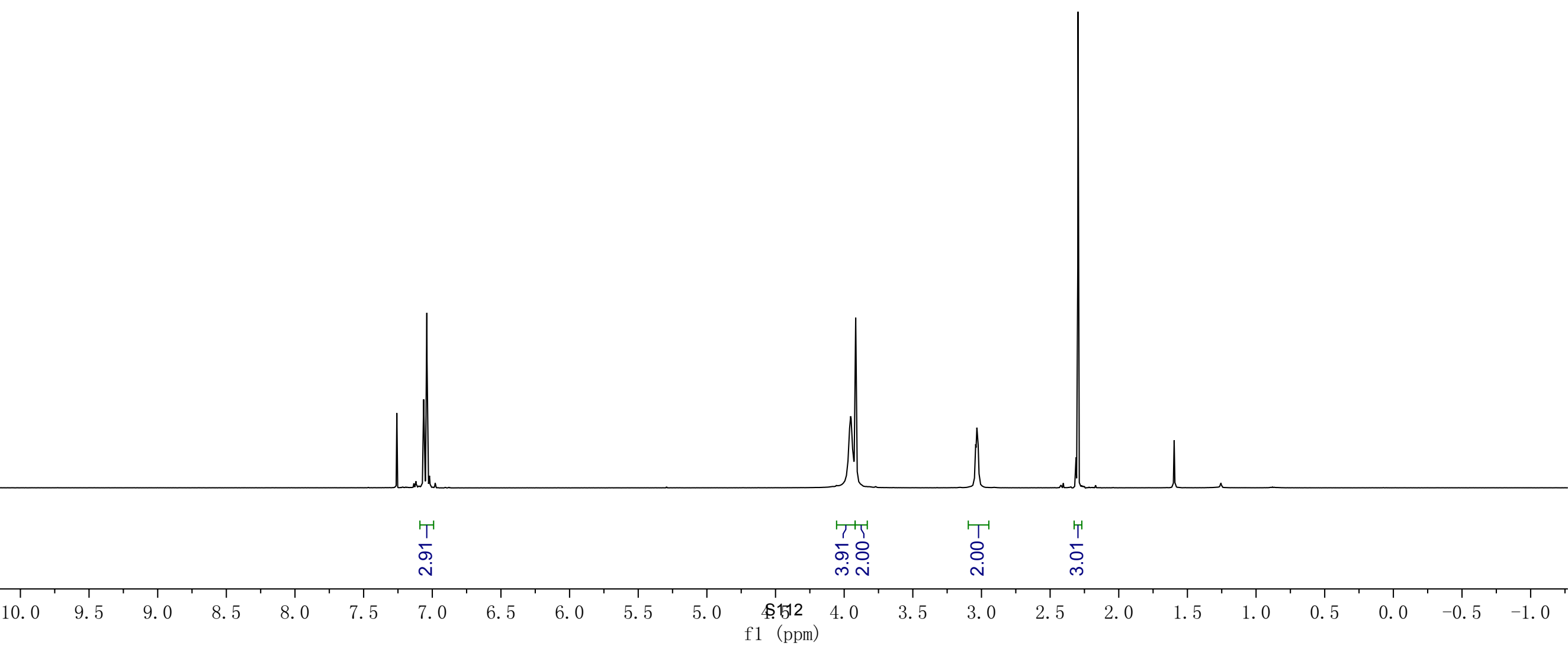




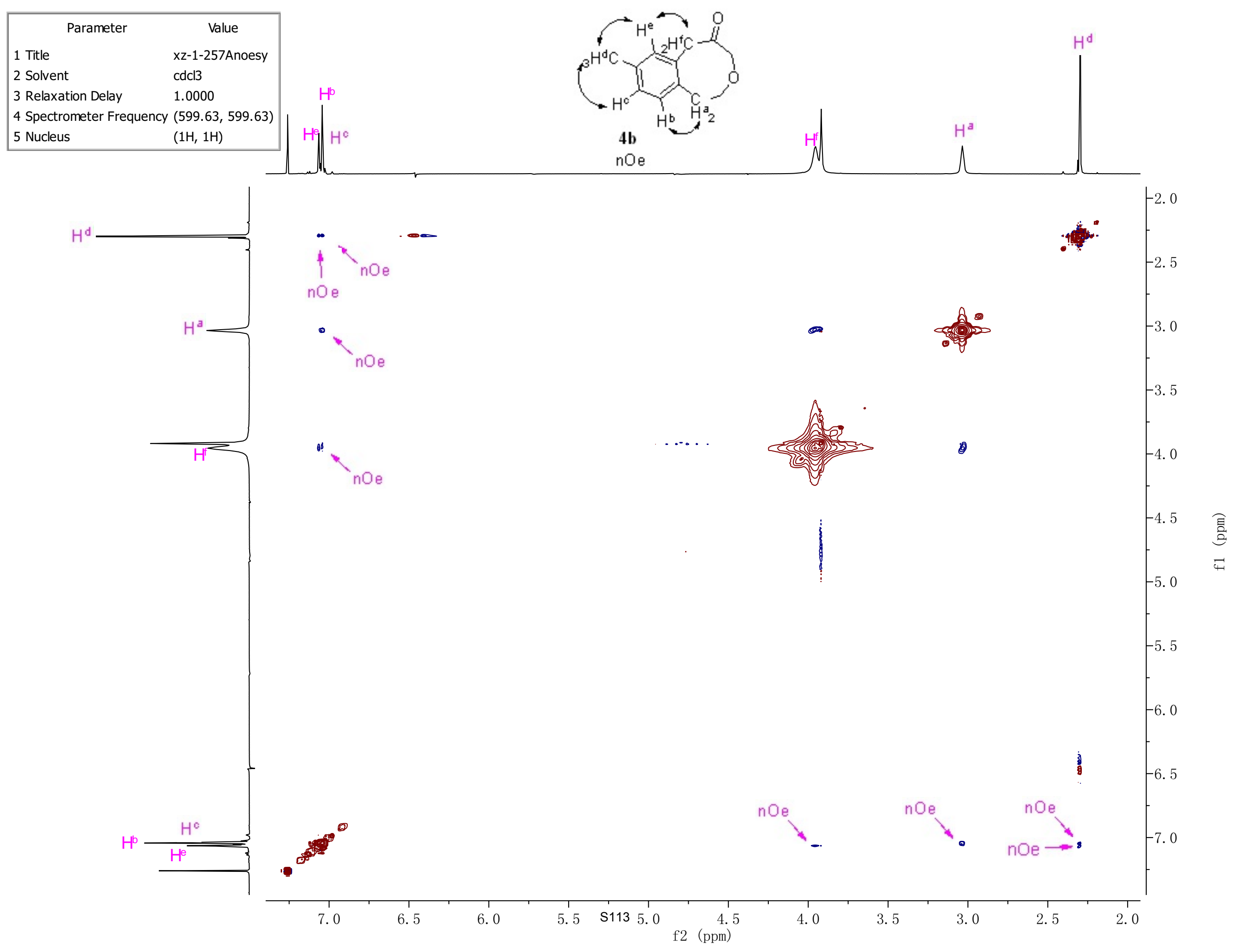




\begin{tabular}{|ll|}
\hline \multicolumn{2}{|c|}{ Parameter } \\
1 Title & $\mathrm{xz}-1$-257ACtotal-500biaopu \\
2 Solvent & $\mathrm{CDCl} 3$ \\
3 Spectrometer Frequency & 125.70 \\
4 Nucleus & $13 \mathrm{C}$ \\
\hline
\end{tabular}

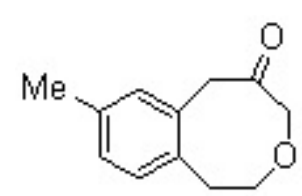

4b

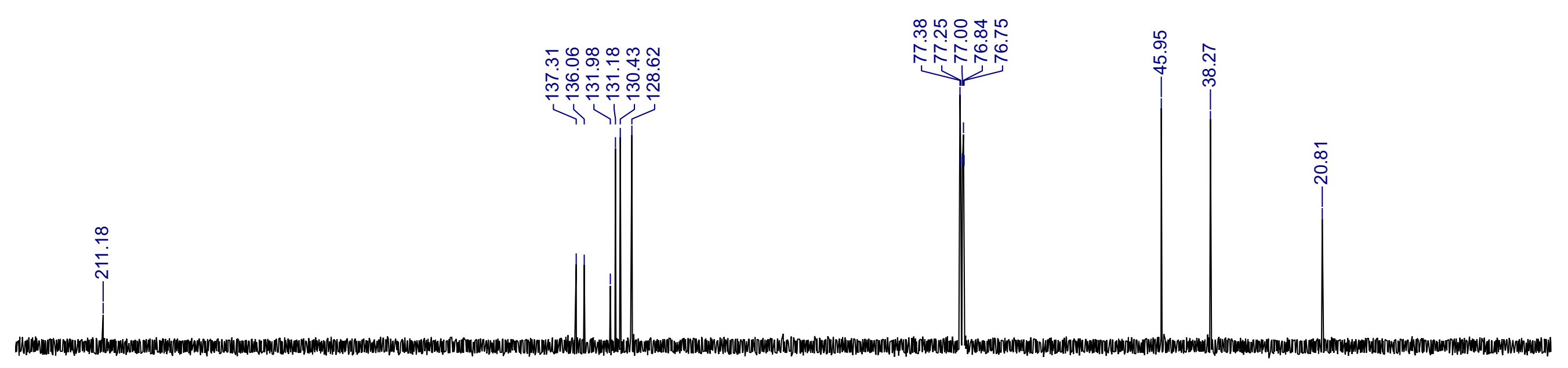

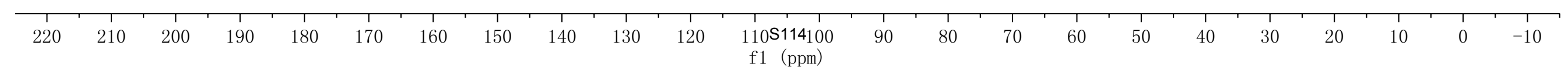




\begin{tabular}{|ll|}
\hline \multicolumn{1}{|c|}{ Parameter } & \multicolumn{1}{c|}{ Value } \\
1 Title & chy-2-304-2-P-1H \\
2 Solvent & $\mathrm{CDCl} 3$ \\
3 Spectrometer Frequency & 499.86 \\
4 Nucleus & $1 \mathrm{H}$ \\
\hline
\end{tabular}
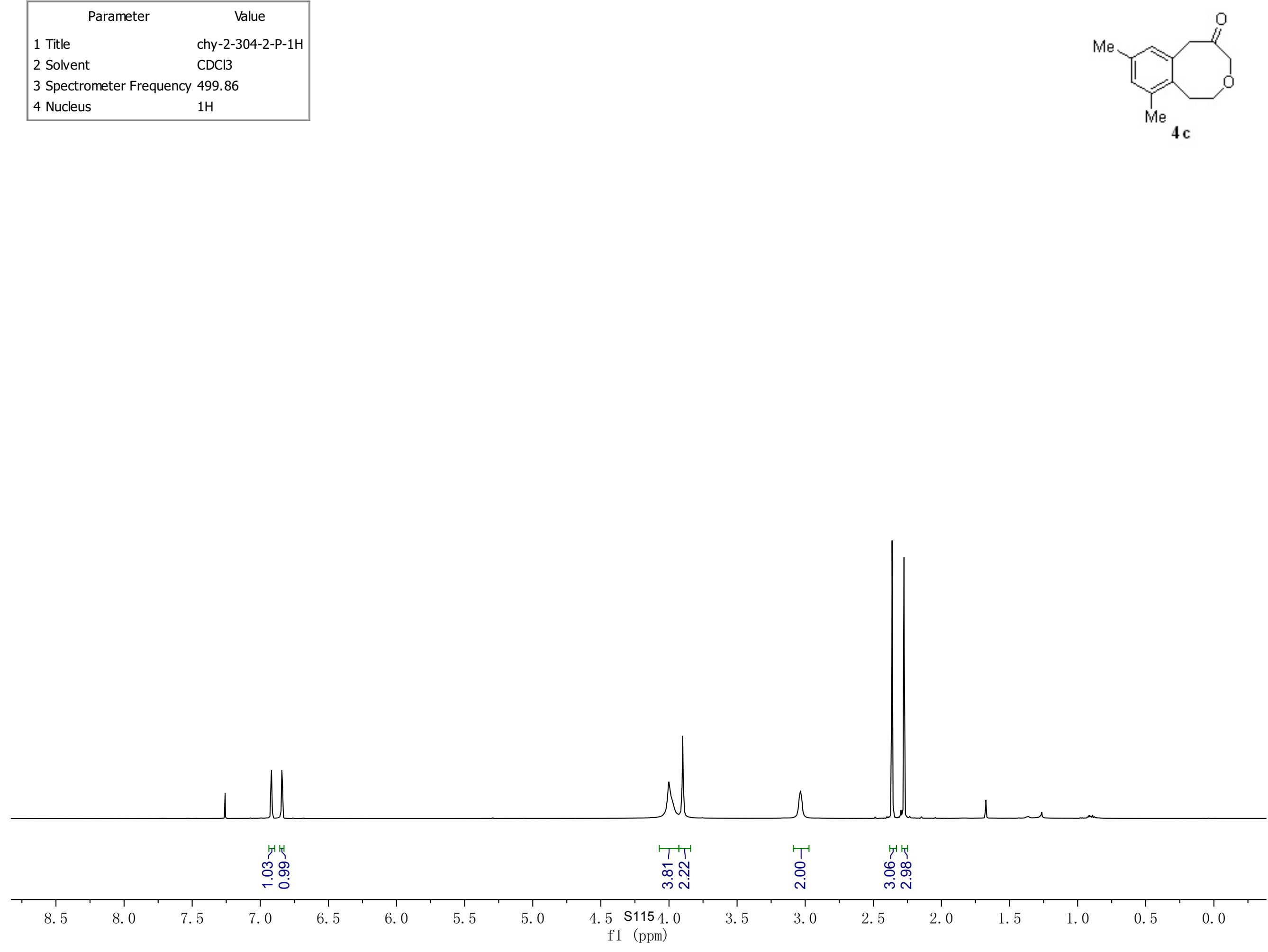


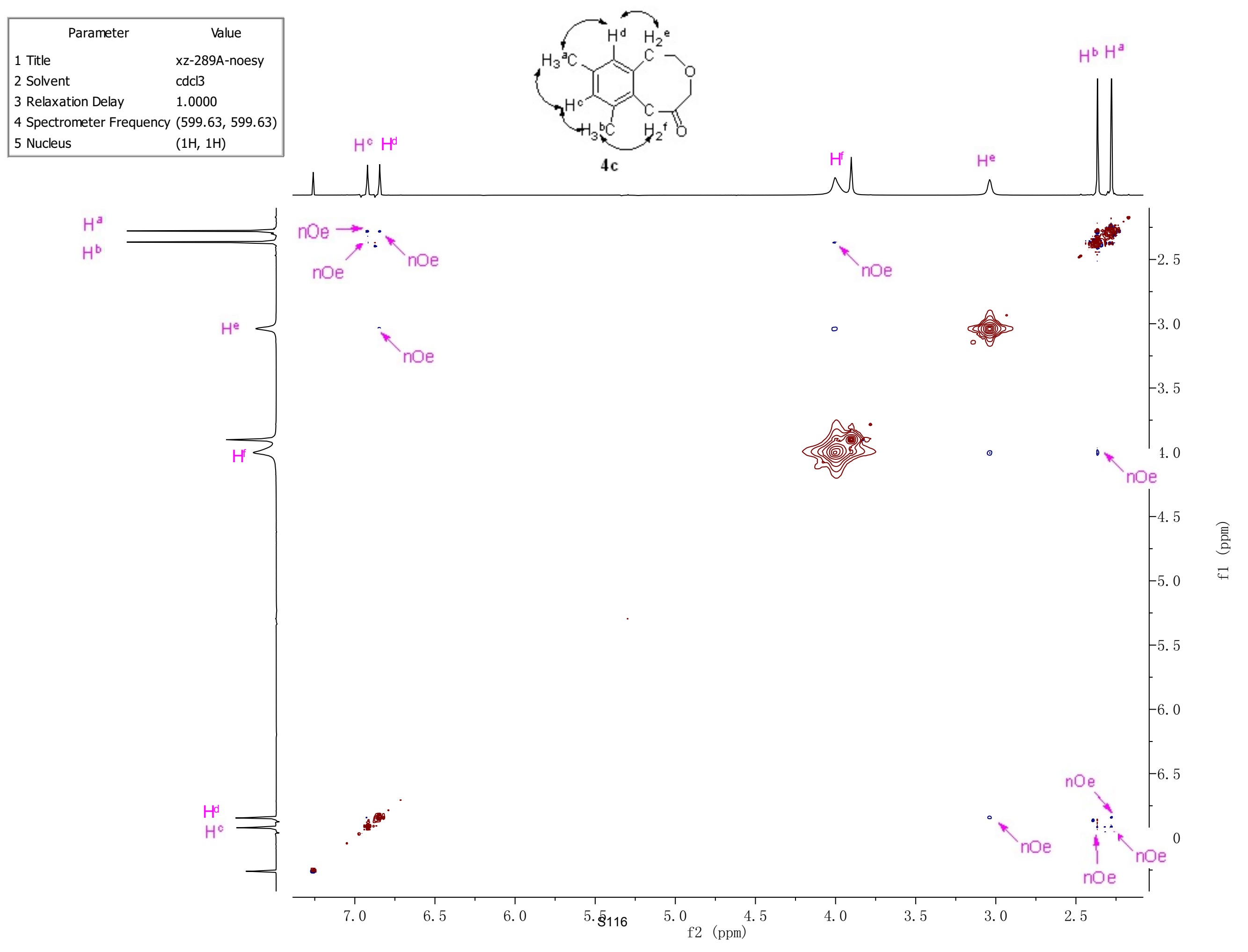




\begin{tabular}{|ll|}
\hline \multicolumn{1}{|c|}{ Parameter } & \multicolumn{1}{c|}{ Value } \\
1 Title & chy-2-304-2-P-13C \\
2 Solvent & CDCl3 \\
3 Spectrometer Frequency & 125.70 \\
4 Nucleus & $13 \mathrm{C}$ \\
\hline
\end{tabular}
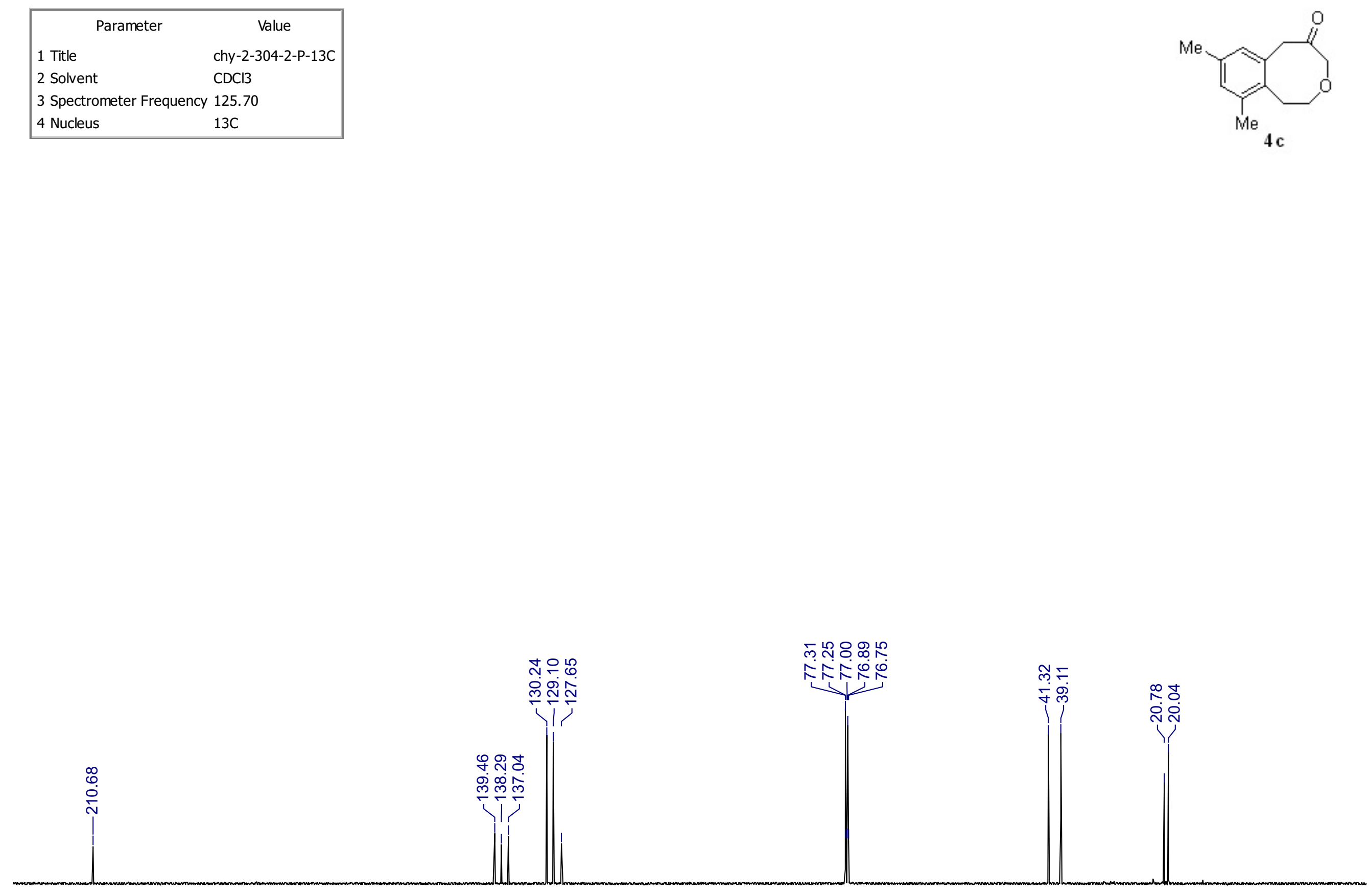

\begin{tabular}{|c|c|c|c|c|c|c|c|c|c|c|c|c|c|c|c|c|c|c|c|c|c|c|}
\hline $\begin{array}{c}1 \\
220\end{array}$ & 210 & $\begin{array}{c}1 \\
200\end{array}$ & $\begin{array}{c}1 \\
190\end{array}$ & $\begin{array}{c}1 \\
180\end{array}$ & $\begin{array}{c}1 \\
170\end{array}$ & $\begin{array}{c}160 \\
160\end{array}$ & $\begin{array}{c} \\
150\end{array}$ & 140 & $\begin{array}{c}1 \\
130\end{array}$ & $\begin{array}{c}12 \\
120\end{array}$ & $\begin{array}{l}110 \mathrm{~S} 117100 \\
\mathrm{f} 1 \mathrm{(ppm})\end{array}$ & $\begin{array}{c}1 \\
90\end{array}$ & 80 & $\begin{array}{l}1 \\
70\end{array}$ & 60 & $\begin{array}{c}1 \\
50\end{array}$ & $\begin{array}{c}1 \\
40\end{array}$ & 30 & $\begin{array}{l}1 \\
20\end{array}$ & 10 & $\begin{array}{l}1 \\
0\end{array}$ & $\begin{array}{c}1 \\
-10\end{array}$ \\
\hline
\end{tabular}




\begin{tabular}{|ll|}
\hline \multicolumn{1}{|c}{ Parameter } & \multicolumn{1}{c|}{ Value } \\
1 Title & $\mathrm{xz}-1-235 \mathrm{CH}$ \\
2 Solvent & $\mathrm{CDCl} 3$ \\
3 Spectrometer Frequency & 499.86 \\
4 Nucleus & $1 \mathrm{H}$ \\
\hline
\end{tabular}
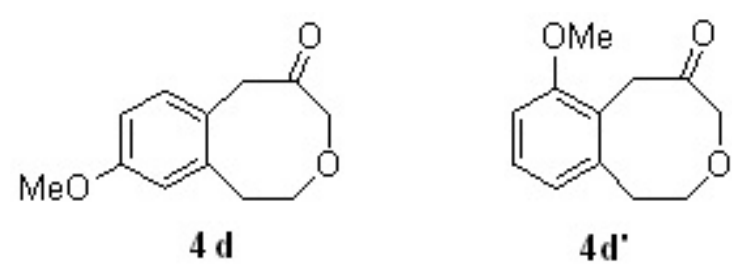

$4 d / 4 d^{\prime}=3 / 1.2$

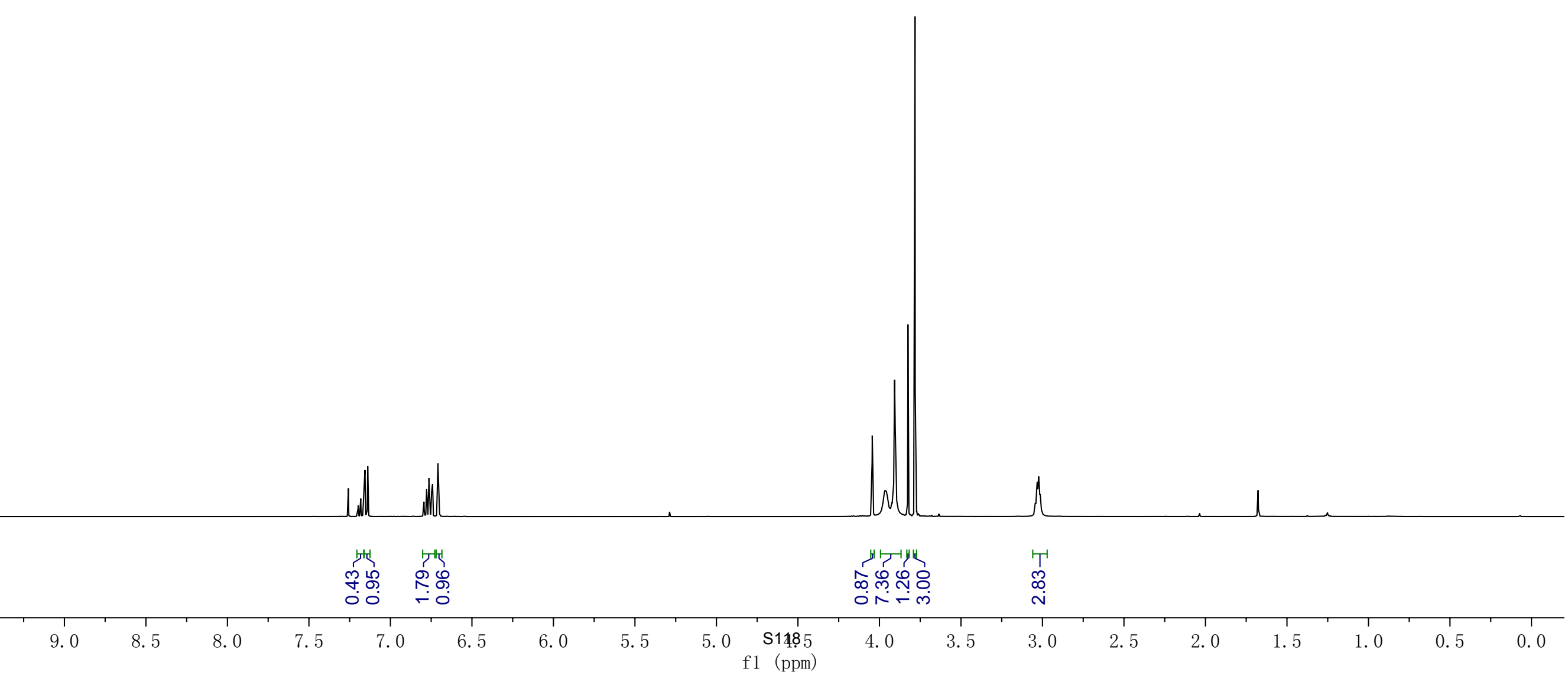




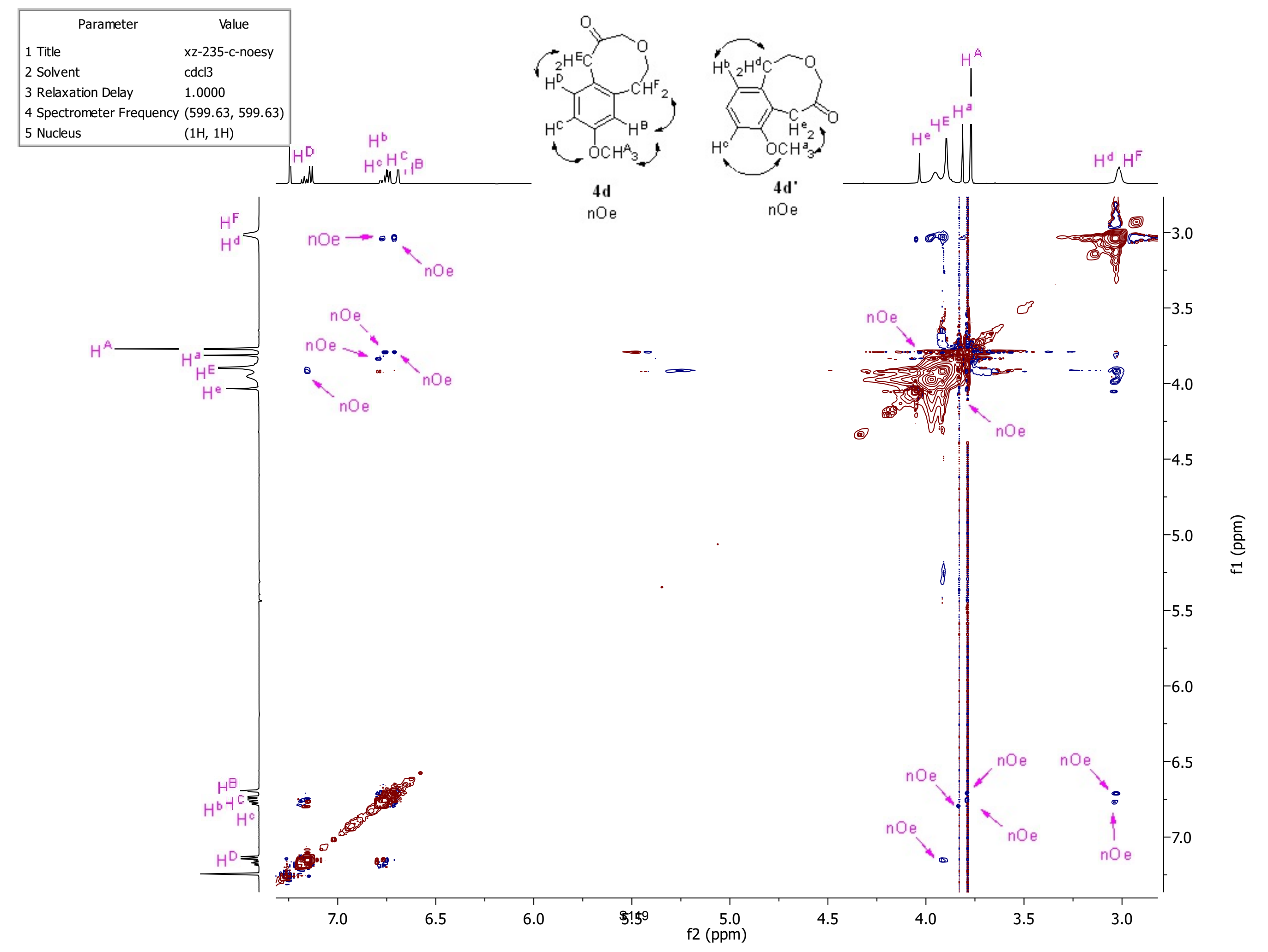




\begin{tabular}{|ll|}
\hline \multicolumn{1}{|c|}{ Parameter } & \multicolumn{1}{c|}{ Value } \\
1 Title & $\mathrm{xz}-1-235$ C500Cbiaopu \\
2 Solvent & $\mathrm{CDCl} 3$ \\
3 Spectrometer Frequency & 125.70 \\
4 Nucleus & $13 \mathrm{C}$ \\
\hline
\end{tabular}
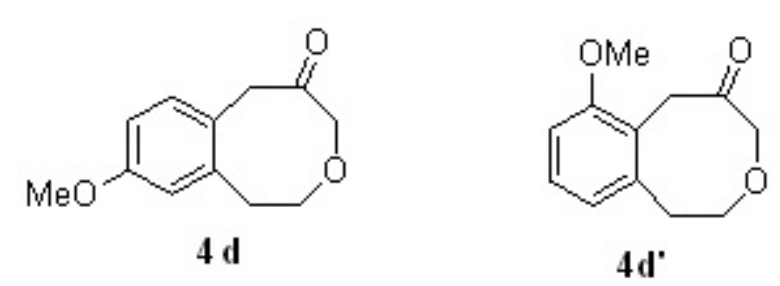

$4 d / 4 d^{\prime}=3 / 1.2$

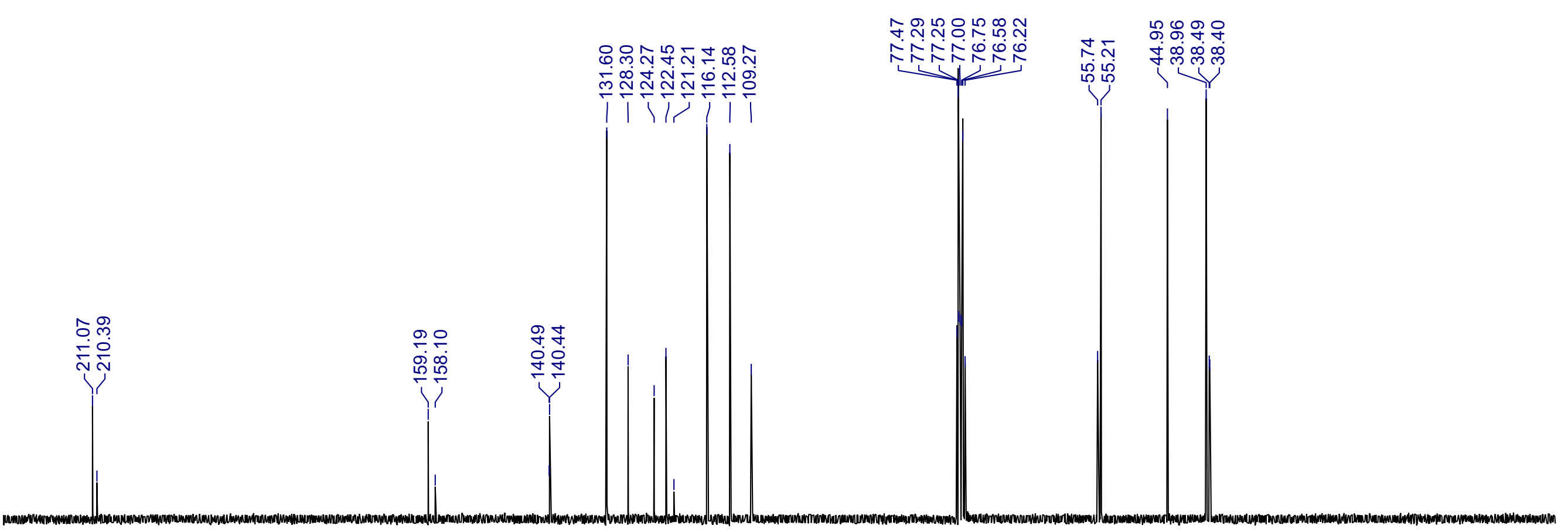

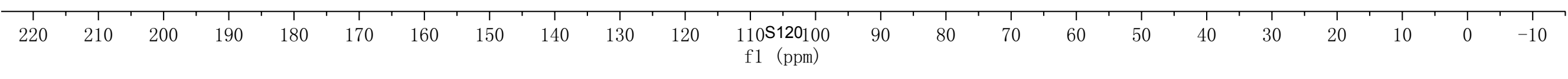




\begin{tabular}{|c|c|}
\hline Parameter & Value \\
\hline 1 Title & xz-1-246A1majorbiaoH-500 \\
\hline 2 Solvent & $\mathrm{CDCl} 3$ \\
\hline 3 Spectrometer Frequenc & 499.86 \\
\hline 4 Nucleus & $1 \mathrm{H}$ \\
\hline
\end{tabular}
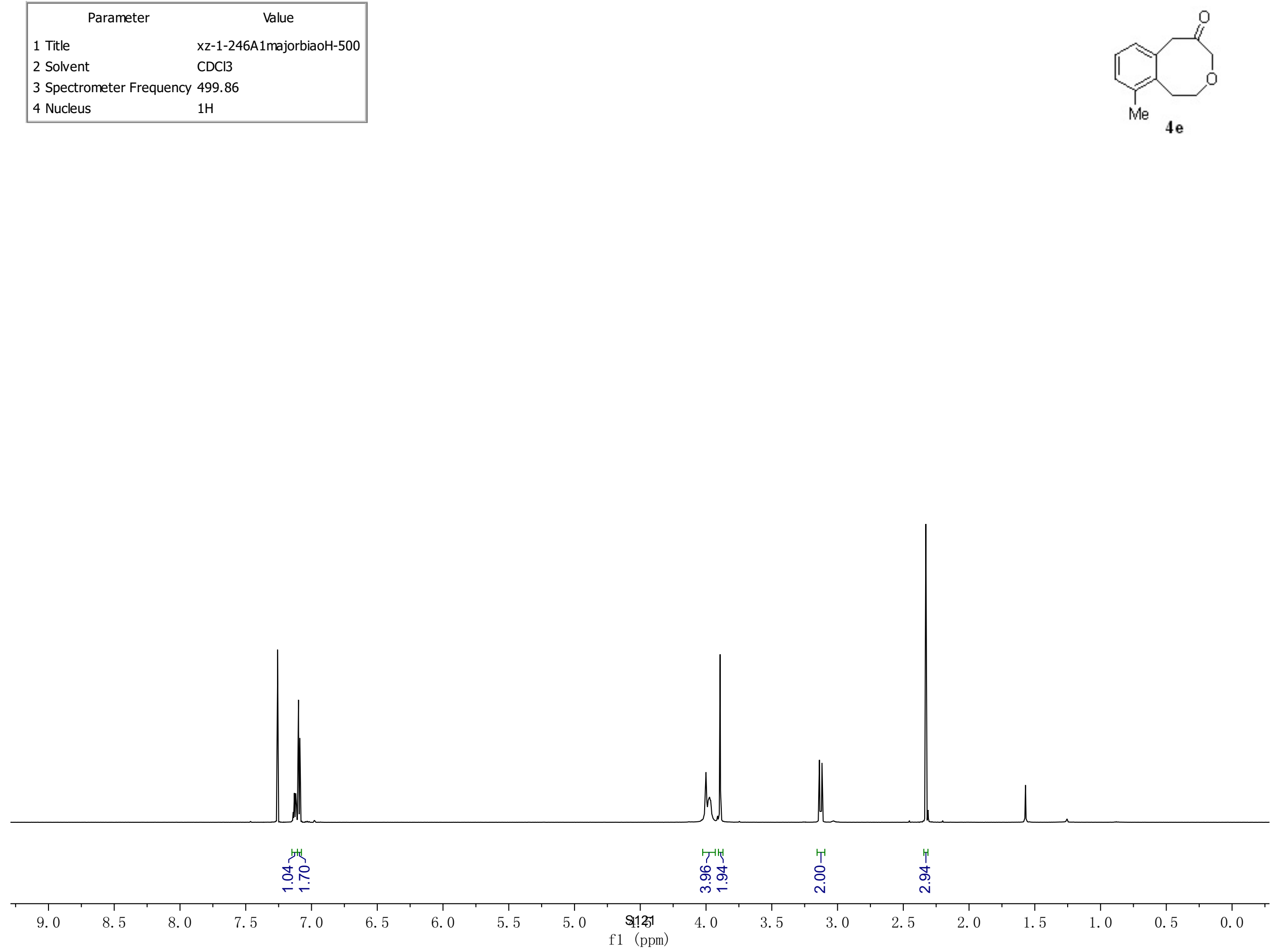


\begin{tabular}{|ll|}
\hline \multicolumn{1}{|c|}{ Parameter } & \multicolumn{1}{c|}{ Value } \\
1 Title & xz-1-244-NOESY \\
2 Solvent & $c d c l 3$ \\
3 Relaxation Delay & 1.0000 \\
4 Spectrometer Frequency & $(599.63,599.63)$ \\
5 Nucleus & $(1 \mathrm{H}, 1 \mathrm{H})$ \\
\hline
\end{tabular}

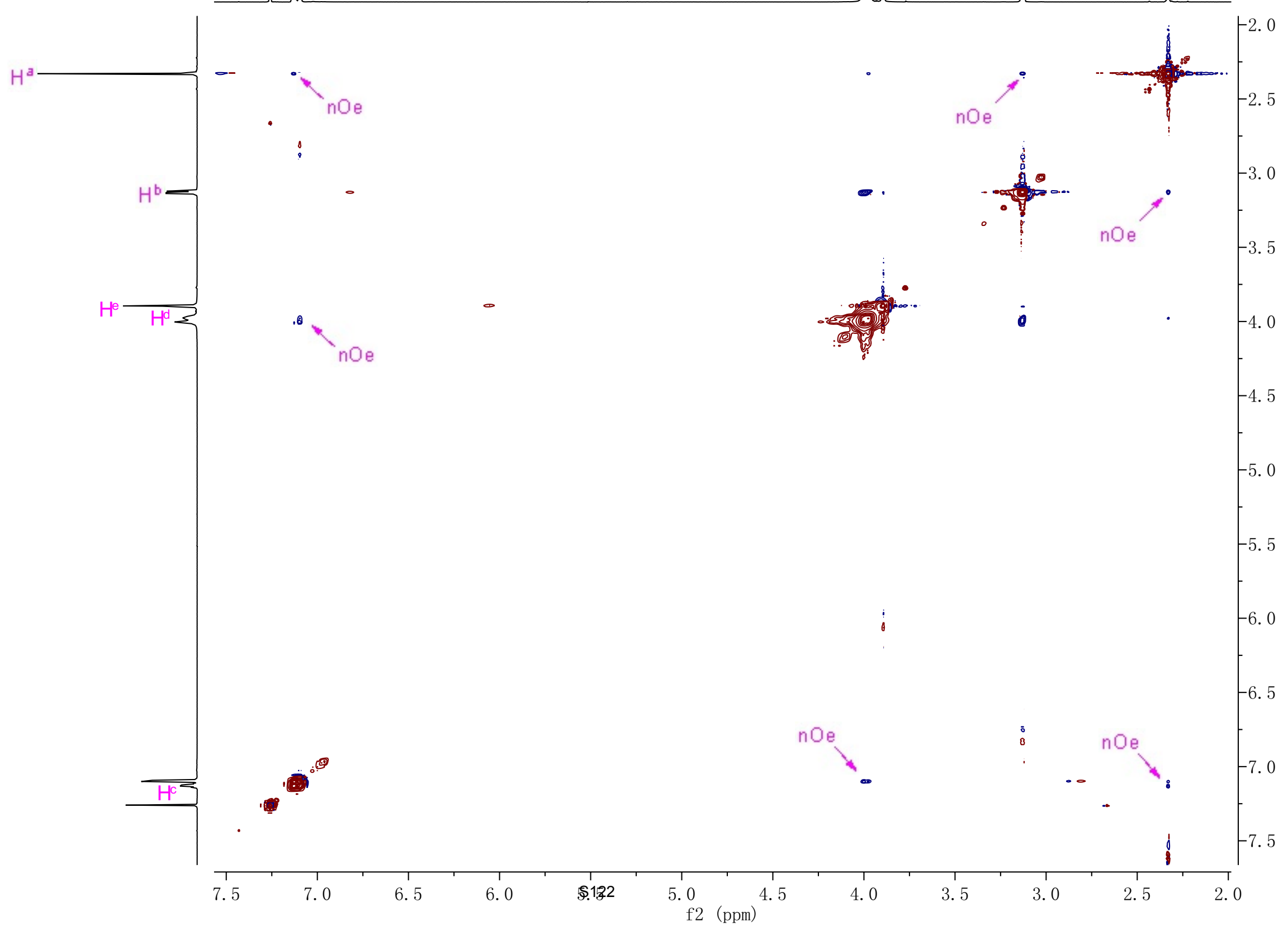




\begin{tabular}{|ll|}
\hline \multicolumn{1}{|c|}{ Parameter } & \multicolumn{1}{c|}{ Value } \\
1 Title & $\mathrm{xz}-1-246 \mathrm{~A} 1 \mathrm{C}-500$ biaopu \\
2 Solvent & $\mathrm{CDCl} 3$ \\
3 Spectrometer Frequency & 125.70 \\
4 Nucleus & $13 \mathrm{C}$ \\
\hline
\end{tabular}
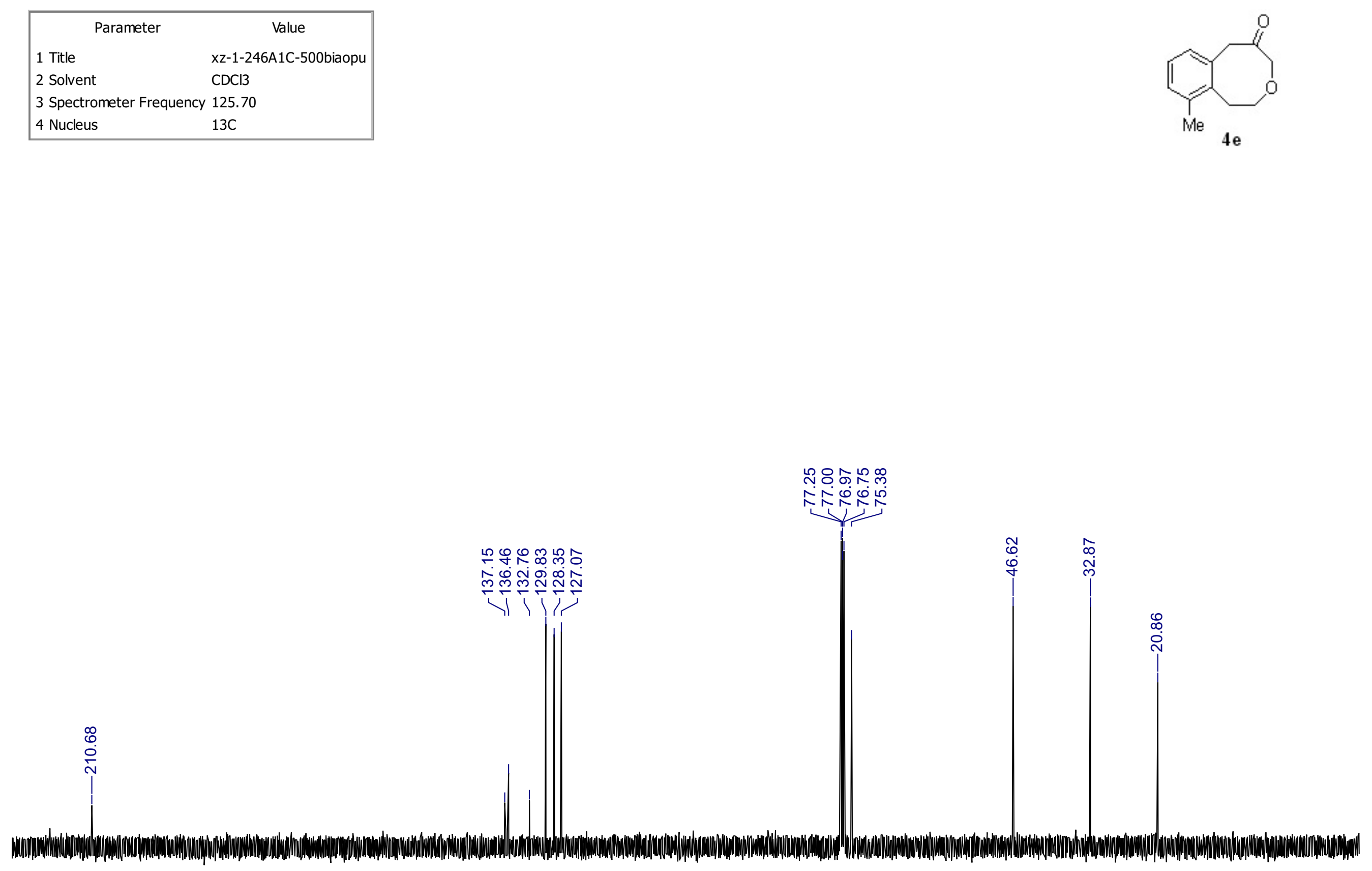

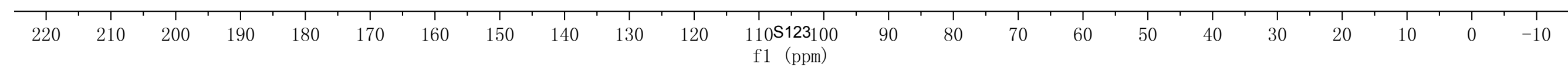




\begin{tabular}{|ll|}
\hline \multicolumn{1}{|c|}{ Parameter } & \multicolumn{1}{c|}{ Value } \\
1 Title & $\mathrm{xz}-1-246 \mathrm{~A} 1 \mathrm{H} 500$ \\
2 Solvent & $\mathrm{CDCl} 3$ \\
3 Spectrometer Frequency & 499.86 \\
4 Nucleus & $1 \mathrm{H}$ \\
\hline
\end{tabular}
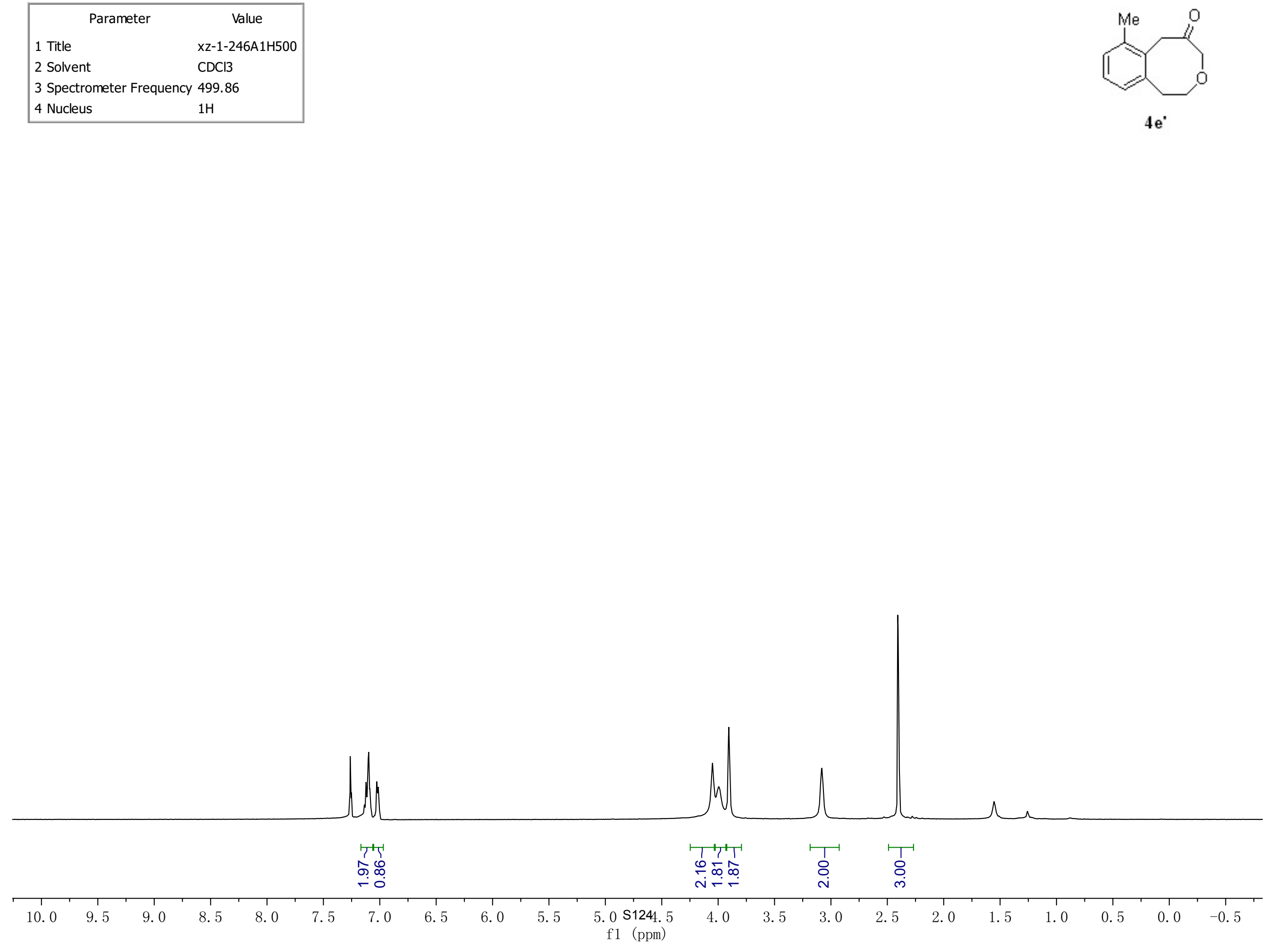


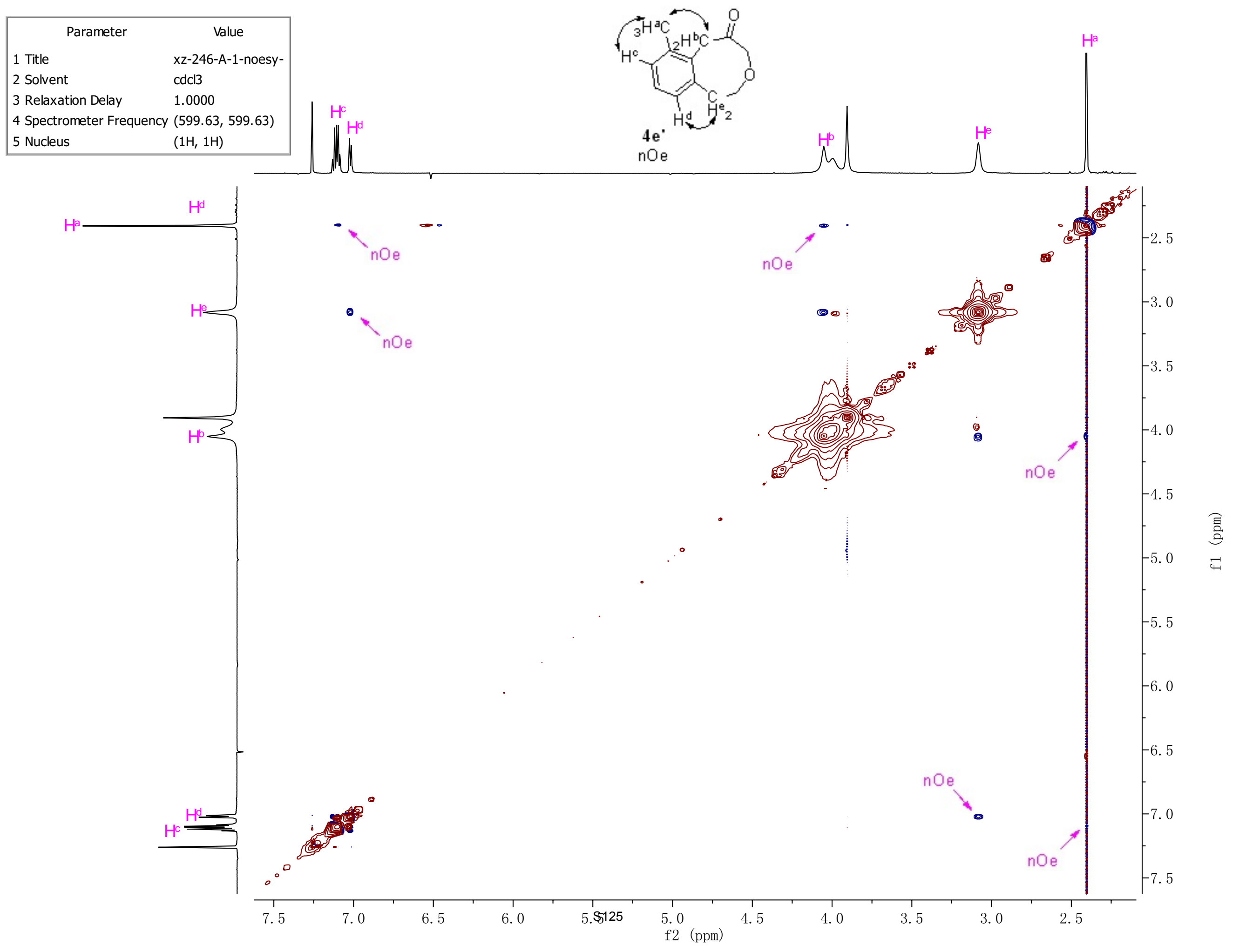




\begin{tabular}{|ll|}
\hline \multicolumn{1}{|c|}{ Parameter } & \multicolumn{1}{c|}{ Value } \\
1 Title & $\mathrm{xz}-1-246 \mathrm{~A} 1 \mathrm{C} 500$ \\
2 Solvent & $\mathrm{CDCl} 3$ \\
3 Spectrometer Frequency & 125.70 \\
4 Nucleus & $13 \mathrm{C}$ \\
\hline
\end{tabular}
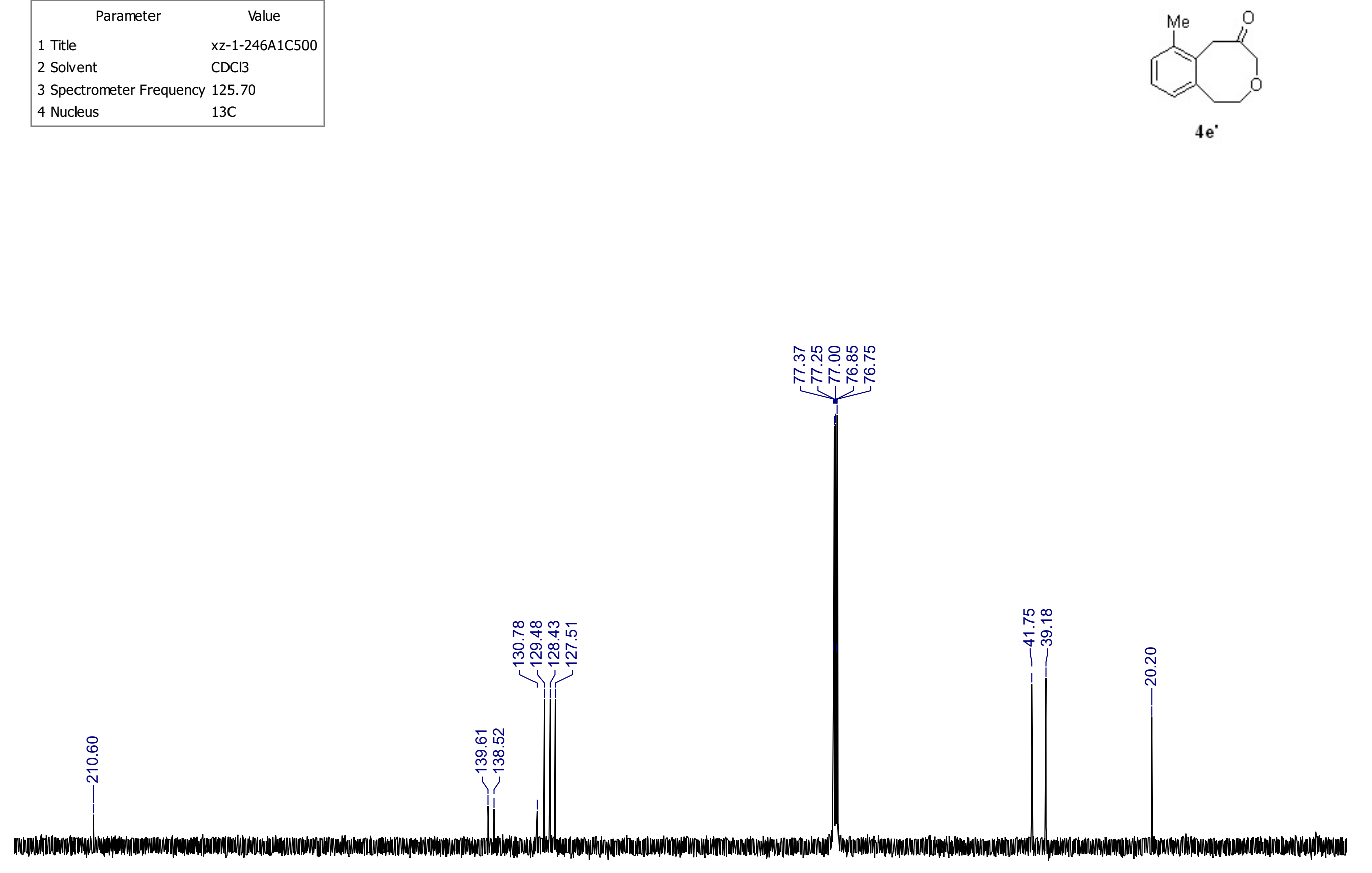

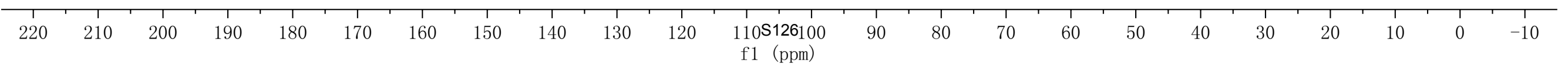




\begin{tabular}{|c|c|}
\hline Parameter & Value \\
\hline 1 Title & xz-1-284AHbiaopu500 \\
\hline 2 Solvent & $\mathrm{CDCl} 3$ \\
\hline 3 Spectrometer Frequency & 499.86 \\
\hline 4 Nucleus & $1 \mathrm{H}$ \\
\hline
\end{tabular}
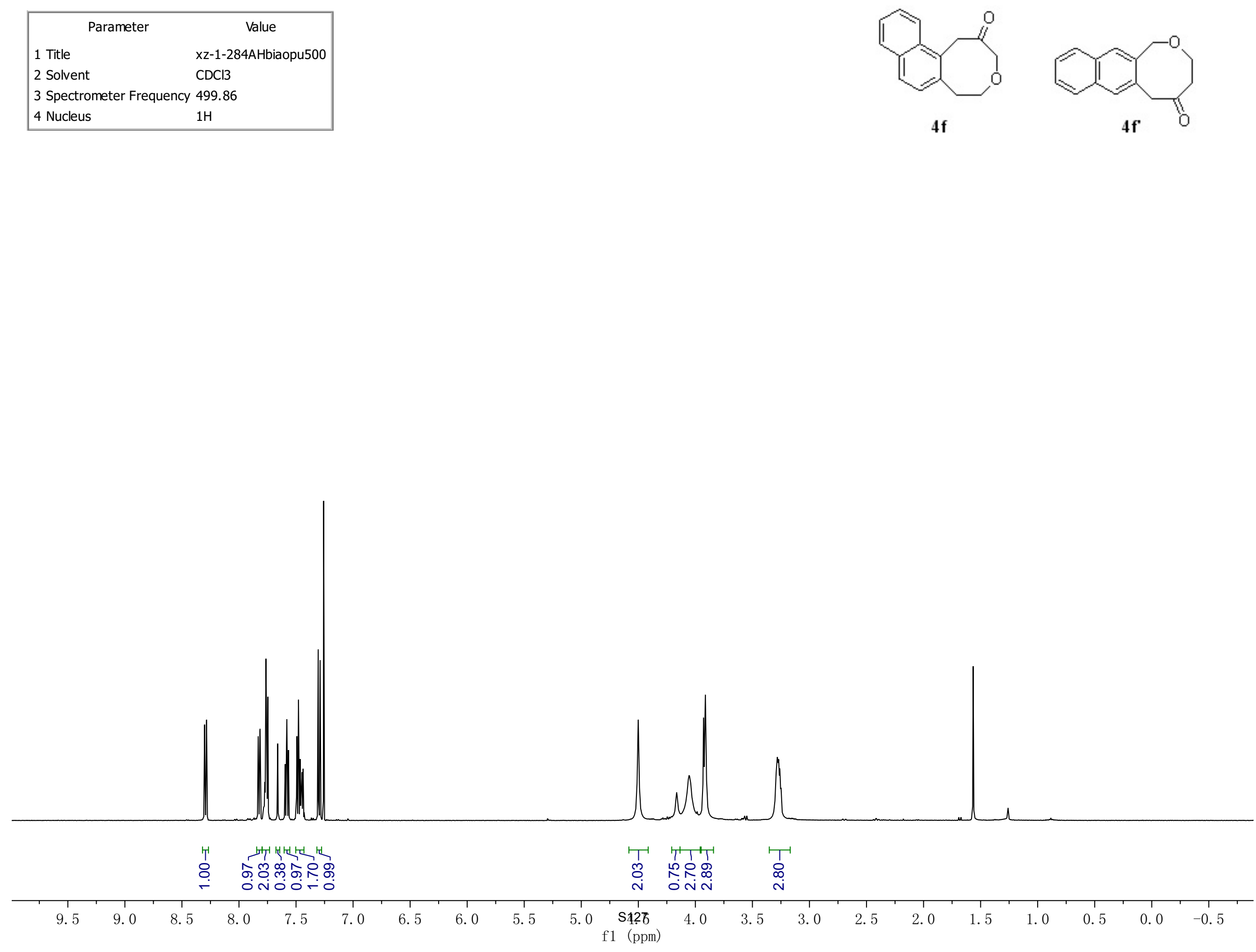


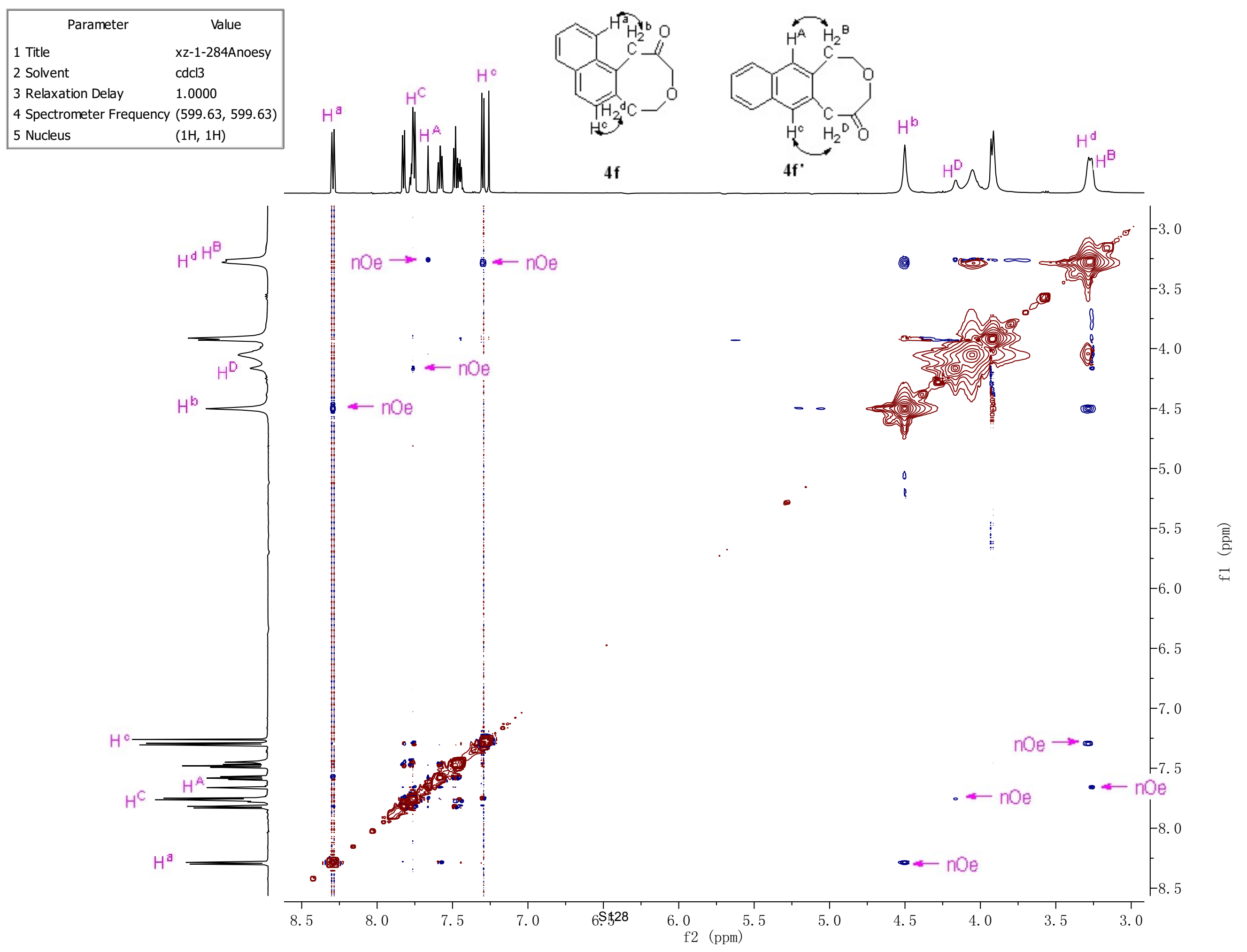




\begin{tabular}{|c|c|}
\hline Parameter & Value \\
\hline 1 Title & xz-1-284AC-500biaopu \\
\hline 2 Solvent & $\mathrm{CDCl} 3$ \\
\hline 3 Spectrometer Frequency & 125.70 \\
\hline 4 Nucleus & $13 C$ \\
\hline
\end{tabular}

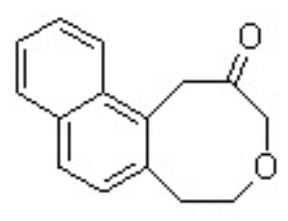

$4 f$

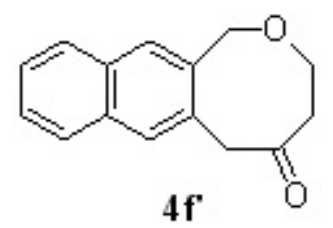

$4 f$

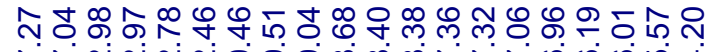

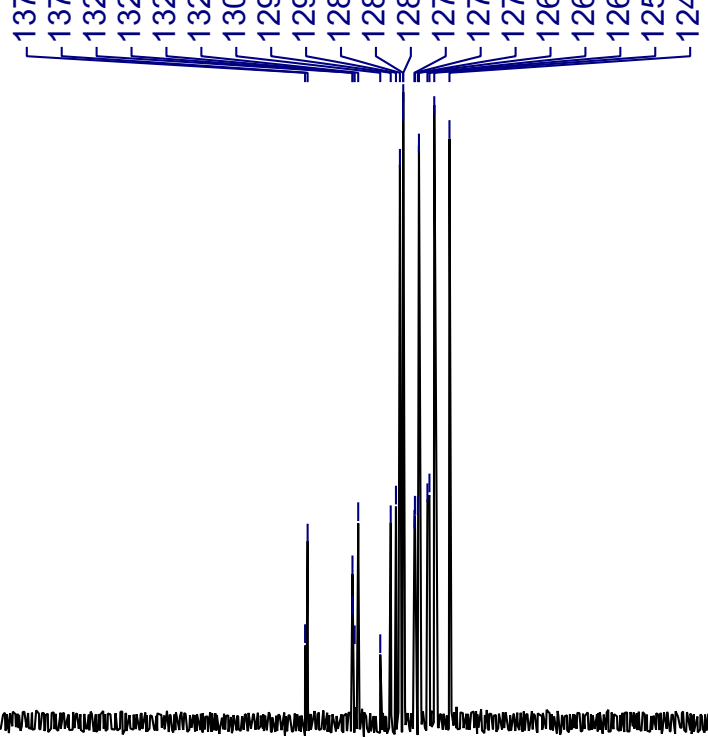

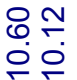

$\bar{N}$
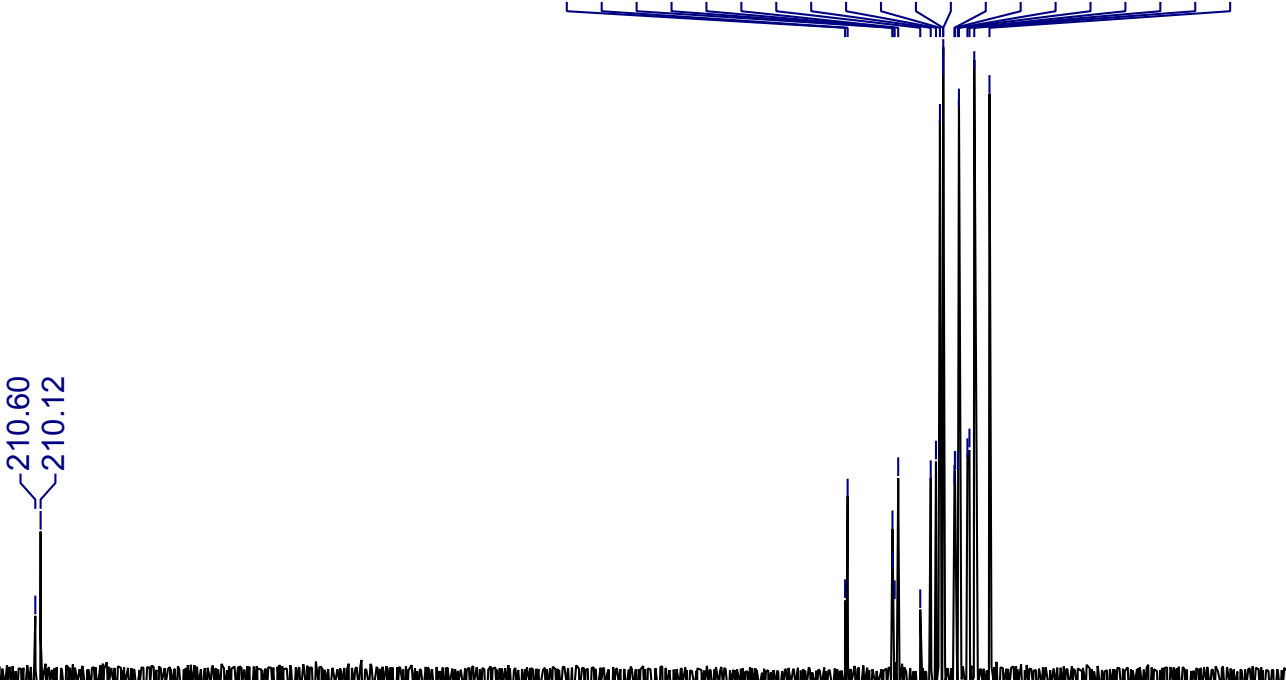

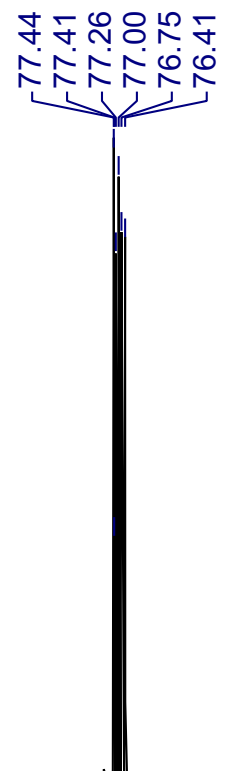

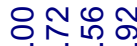

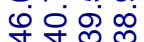

रो

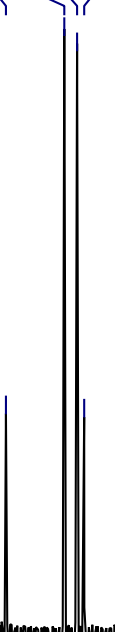

\begin{tabular}{|c|c|c|c|c|c|c|c|c|c|c|c|c|c|c|c|c|c|c|c|c|c|c|}
\hline 220 & 210 & 200 & 190 & 180 & 170 & 160 & 150 & 140 & 130 & 120 & $\begin{array}{l}110 S 129100 \\
\text { f1 (ppm) }\end{array}$ & 90 & 80 & 70 & 60 & 50 & 40 & 30 & 20 & 10 & 0 & -10 \\
\hline
\end{tabular}




\begin{tabular}{|ll|}
\hline \multicolumn{1}{|c|}{ Parameter } & \multicolumn{1}{c|}{ Value } \\
1 Title & $\mathrm{xz}-1-282 \mathrm{BB} 1 \mathrm{Hbiaopu} 500$ \\
2 Solvent & $\mathrm{CDCl} 3$ \\
3 Spectrometer Frequency & 499.86 \\
4 Nucleus & $1 \mathrm{H}$ \\
\hline
\end{tabular}
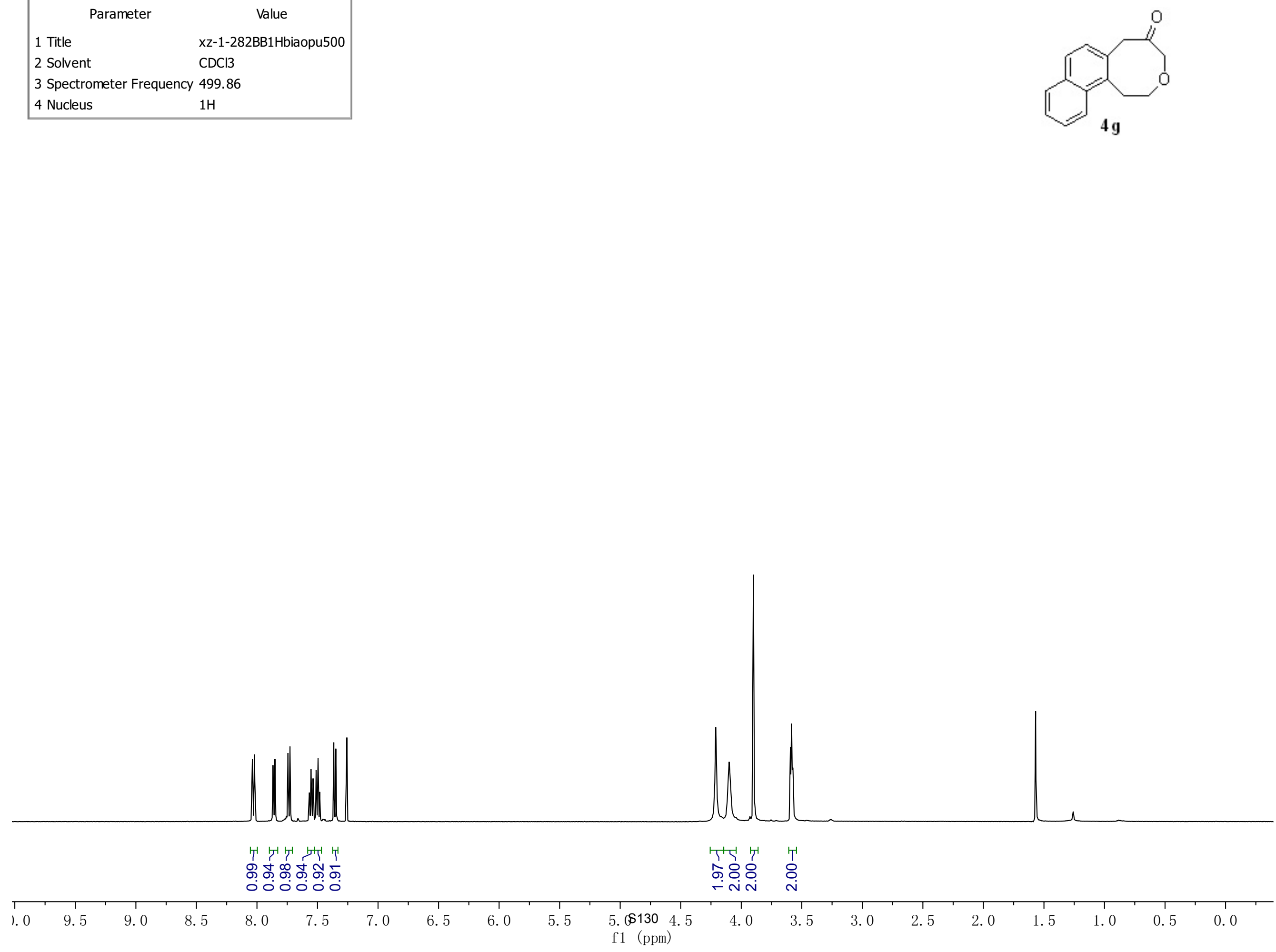


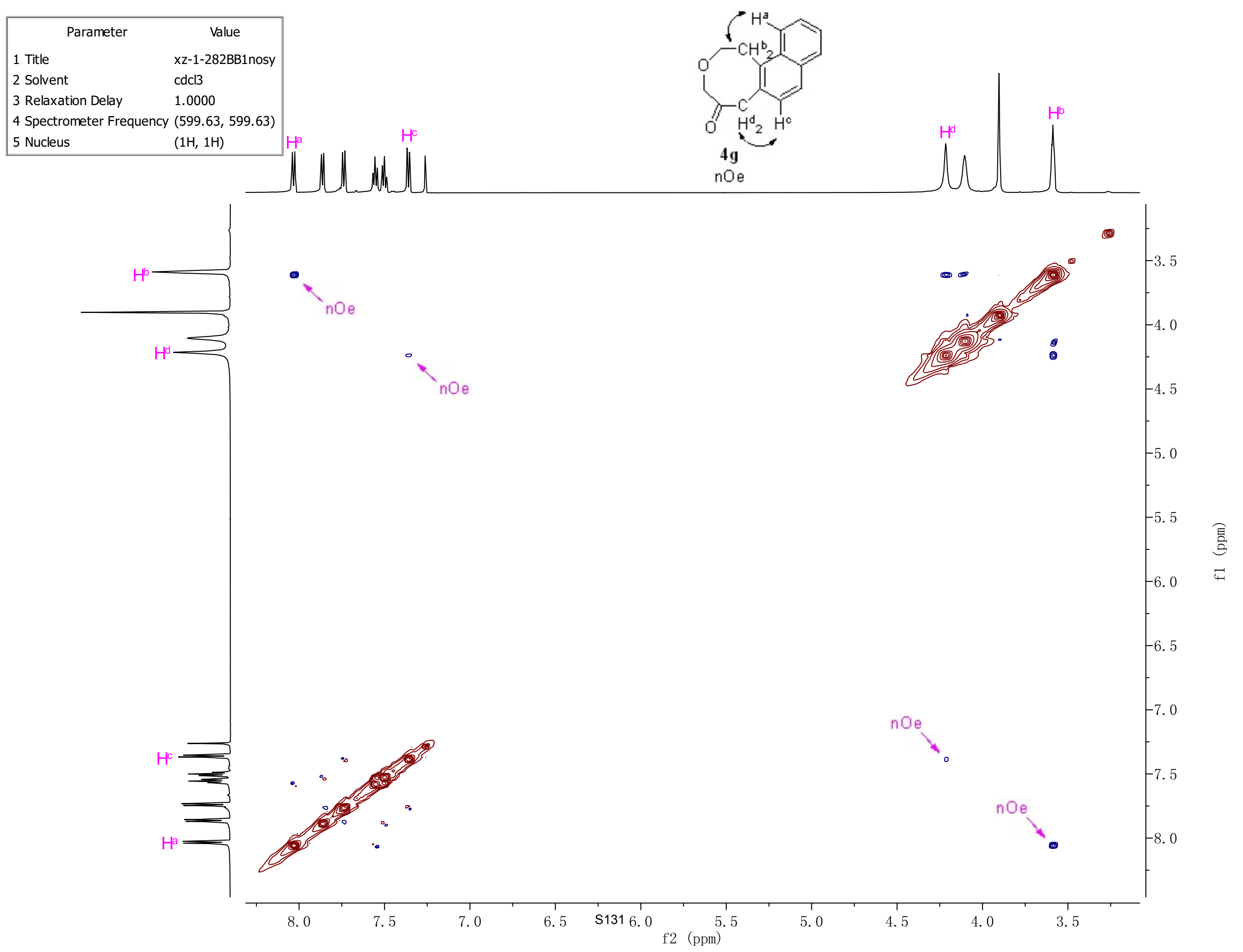



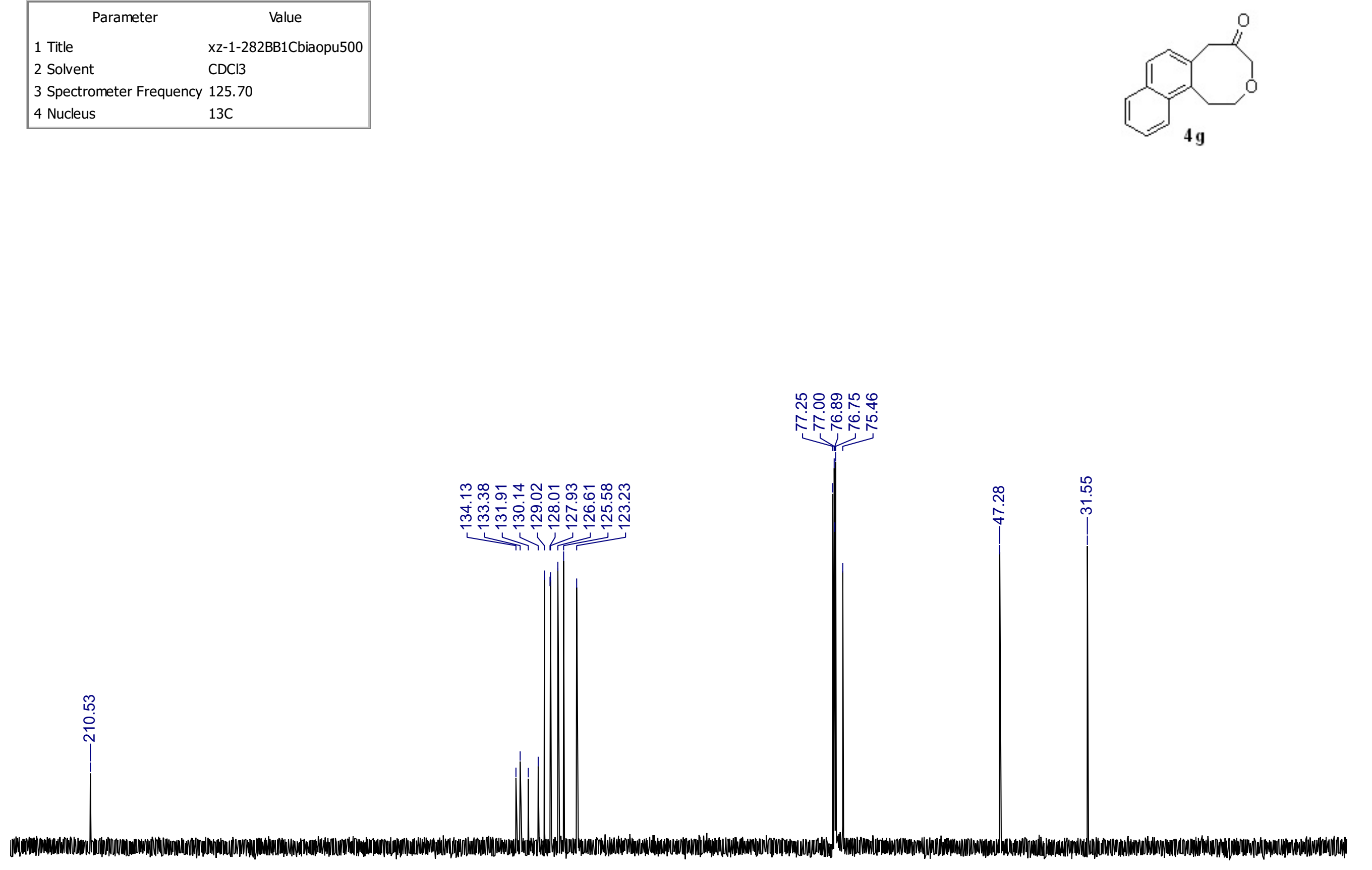

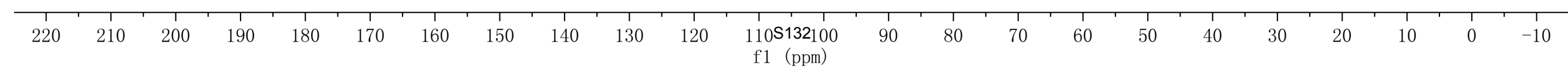




\begin{tabular}{|c|c|}
\hline Parameter & Value \\
\hline 1 Title & $x z-1-282 B B 2 H$ \\
\hline 2 Solvent & $\mathrm{CDCl} 3$ \\
\hline 3 Spectrometer Frequenc & 499.86 \\
\hline 4 Nucleus & $1 \mathrm{H}$ \\
\hline
\end{tabular}
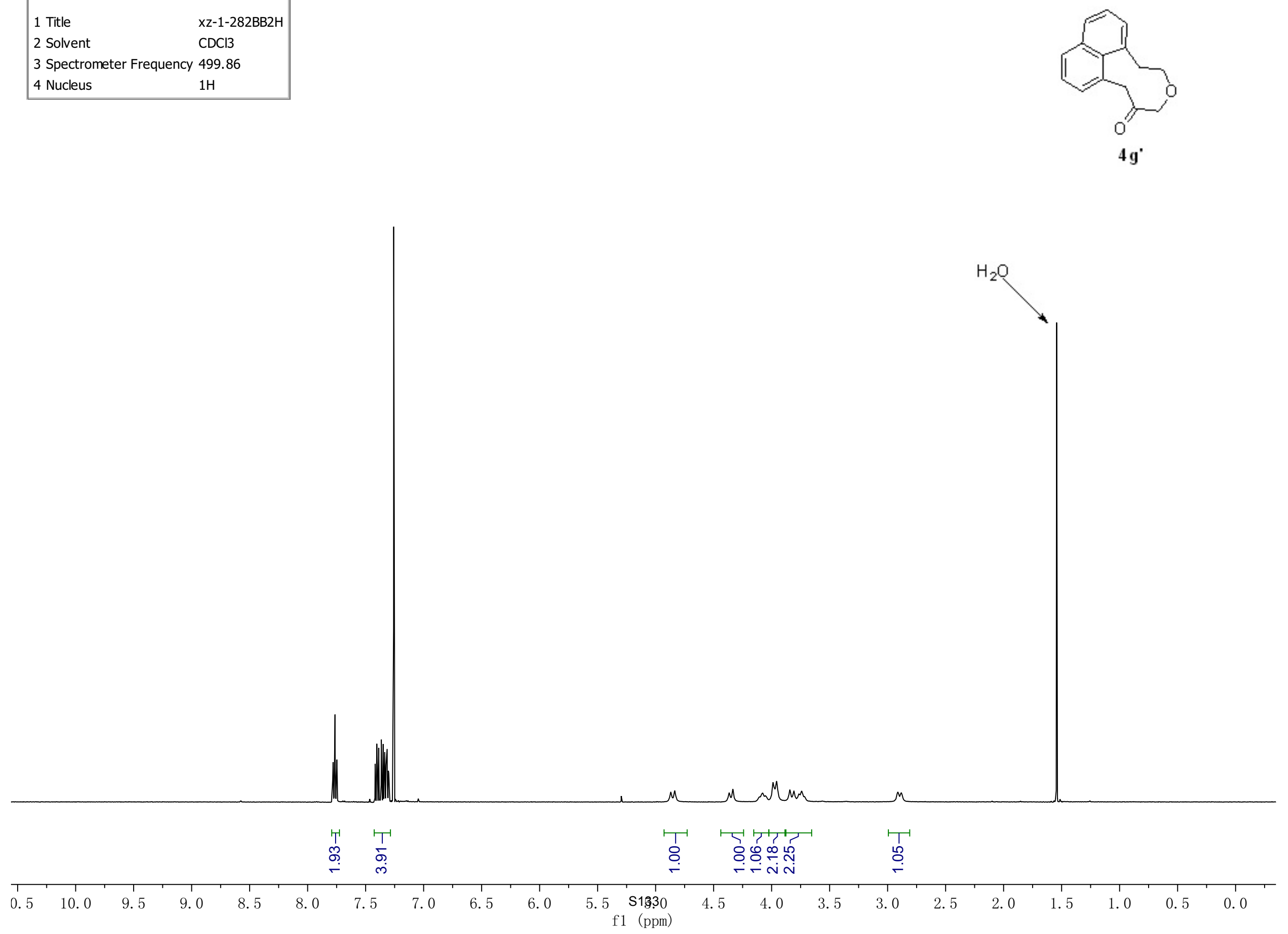


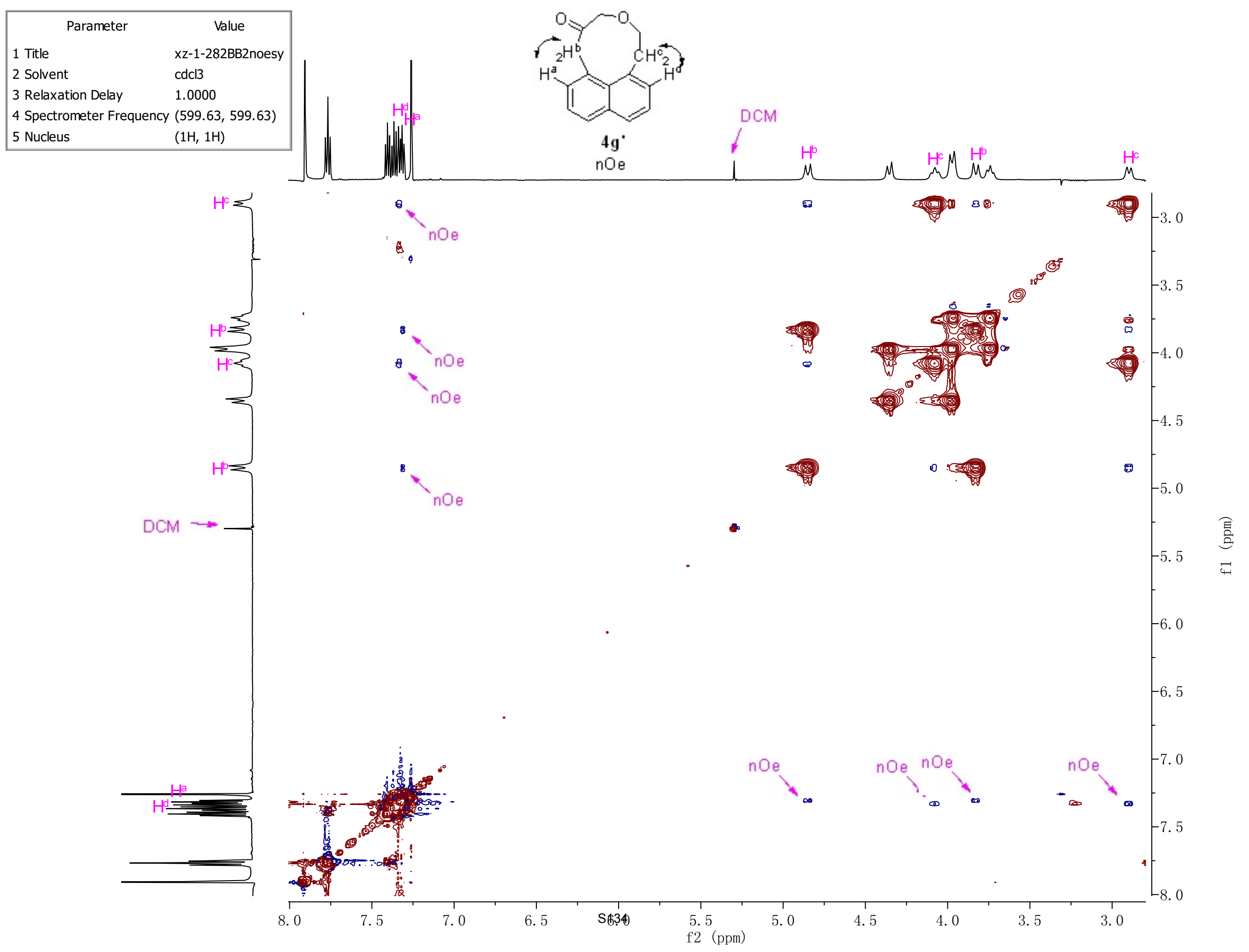




\begin{tabular}{|ll|}
\hline \multicolumn{1}{|c|}{ Parameter } & \multicolumn{1}{c|}{ Value } \\
1 Title & $\mathrm{xz}-1-282 \mathrm{BB} 2-500 \mathrm{Cbiaopu}$ \\
2 Solvent & $\mathrm{CDCl} 3$ \\
3 Spectrometer Frequency & 125.70 \\
4 Nucleus & $13 \mathrm{C}$ \\
\hline
\end{tabular}

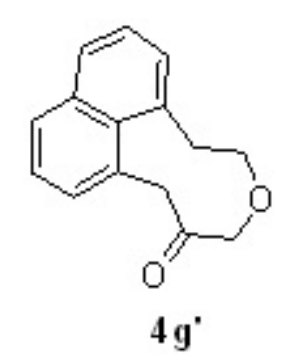

œ

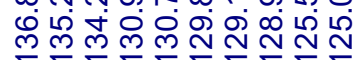
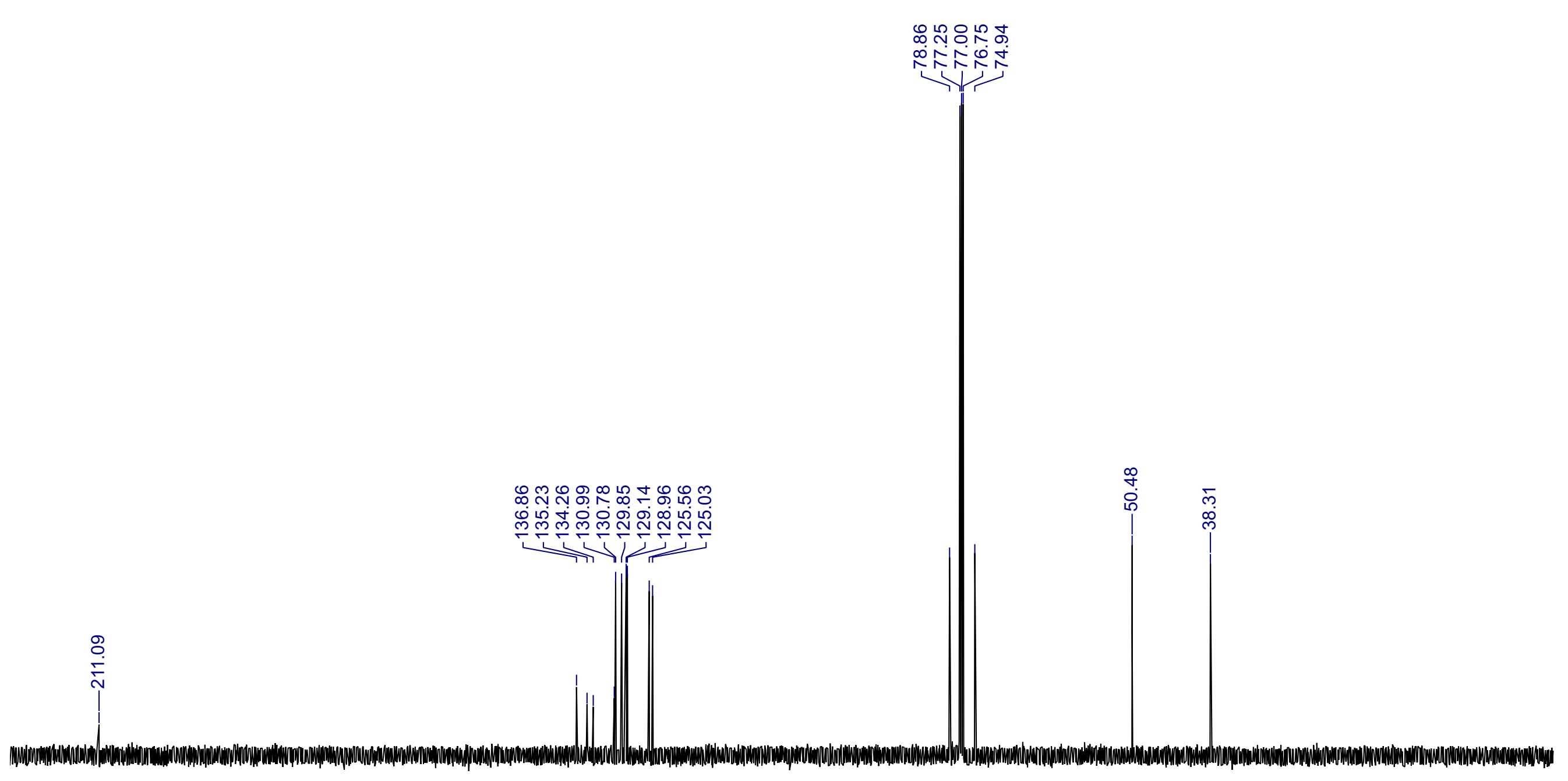

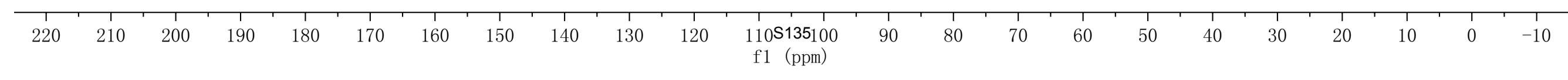




\begin{tabular}{|ll|}
\hline \multicolumn{1}{|c|}{ Parameter } & \multicolumn{1}{c|}{ Value } \\
1 Title & chy-2-295-2-P-1H \\
2 Solvent & CDCl3 \\
3 Spectrometer Frequency & 499.86 \\
4 Nucleus & $1 \mathrm{H}$ \\
\hline
\end{tabular}

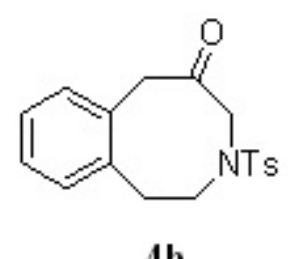

4h

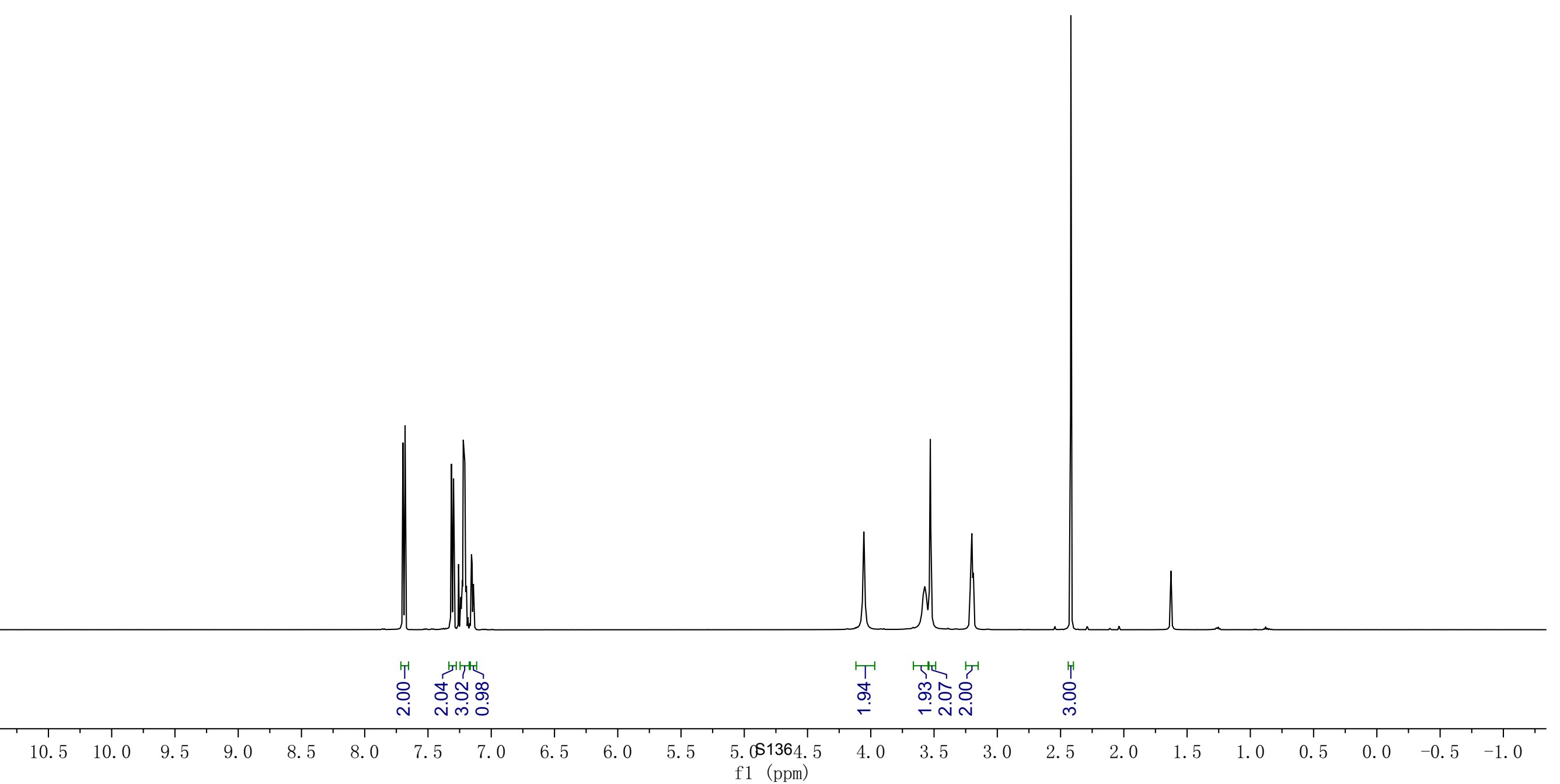




\begin{tabular}{|ll|}
\hline \multicolumn{1}{|c|}{ Parameter } & \multicolumn{1}{c|}{ Value } \\
1 Title & chy-2-295-2-P-13C \\
2 Solvent & CDCl3 \\
3 Spectrometer Frequency & 125.70 \\
4 Nucleus & $13 \mathrm{C}$ \\
\hline
\end{tabular}

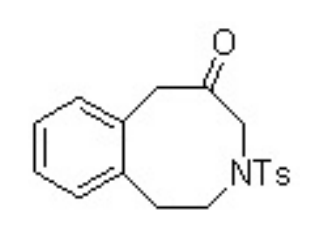

๓

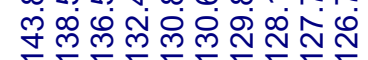

还

$\stackrel{\circ}{\circ}$
仓்
N 


\begin{tabular}{|ll|}
\hline \multicolumn{1}{|c|}{ Parameter } & \multicolumn{1}{c|}{ Value } \\
1 Title & chy-3-144-2-P-1H \\
2 Solvent & $\mathrm{CDCl} 3$ \\
3 Spectrometer Frequency & 499.86 \\
4 Nucleus & $1 \mathrm{H}$ \\
\hline
\end{tabular}

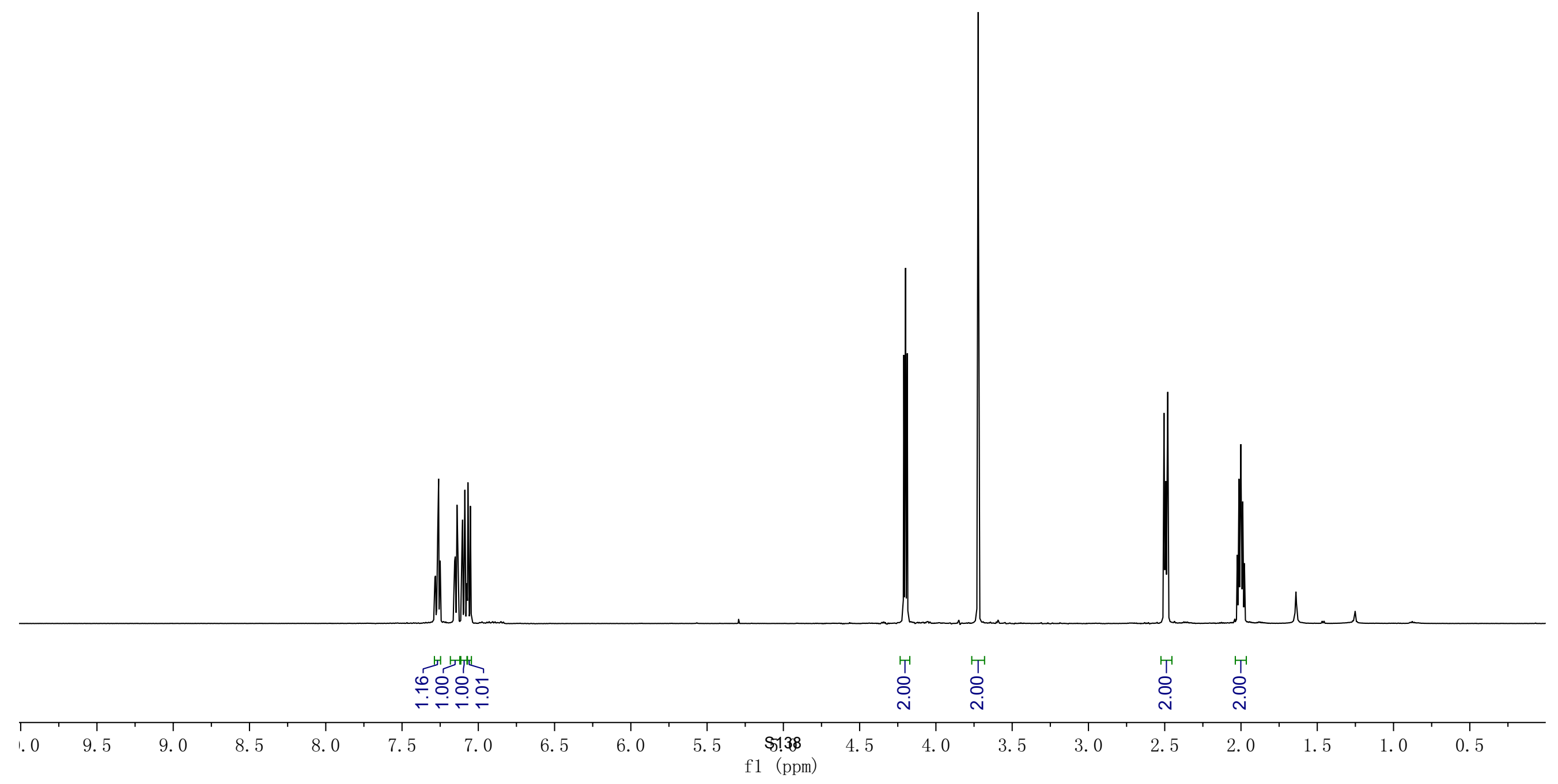


3 Spectrometer Frequency 125.70

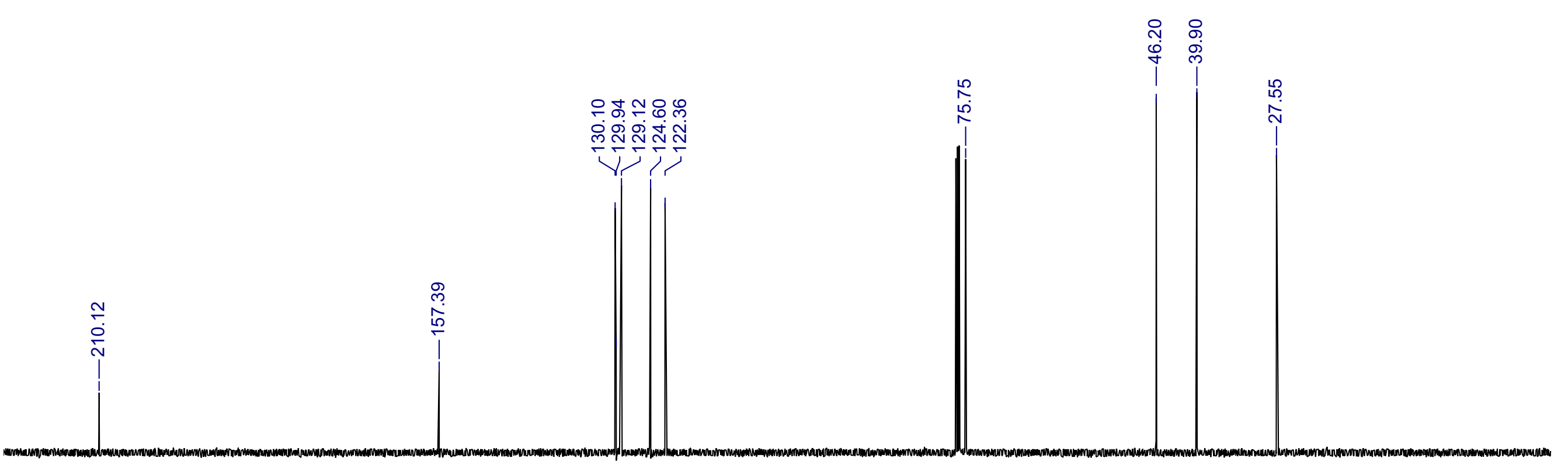

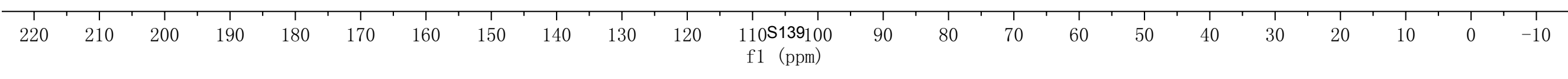




\begin{tabular}{|ll|}
\hline \multicolumn{1}{|c|}{ Parameter } & \multicolumn{1}{c|}{ Value } \\
1 Title & $\mathrm{xz}-1-241 \mathrm{CH} 500$ \\
2 Solvent & $\mathrm{CDCl} 3$ \\
3 Spectrometer Frequency & 499.86 \\
4 Nucleus & $1 \mathrm{H}$ \\
\hline
\end{tabular}

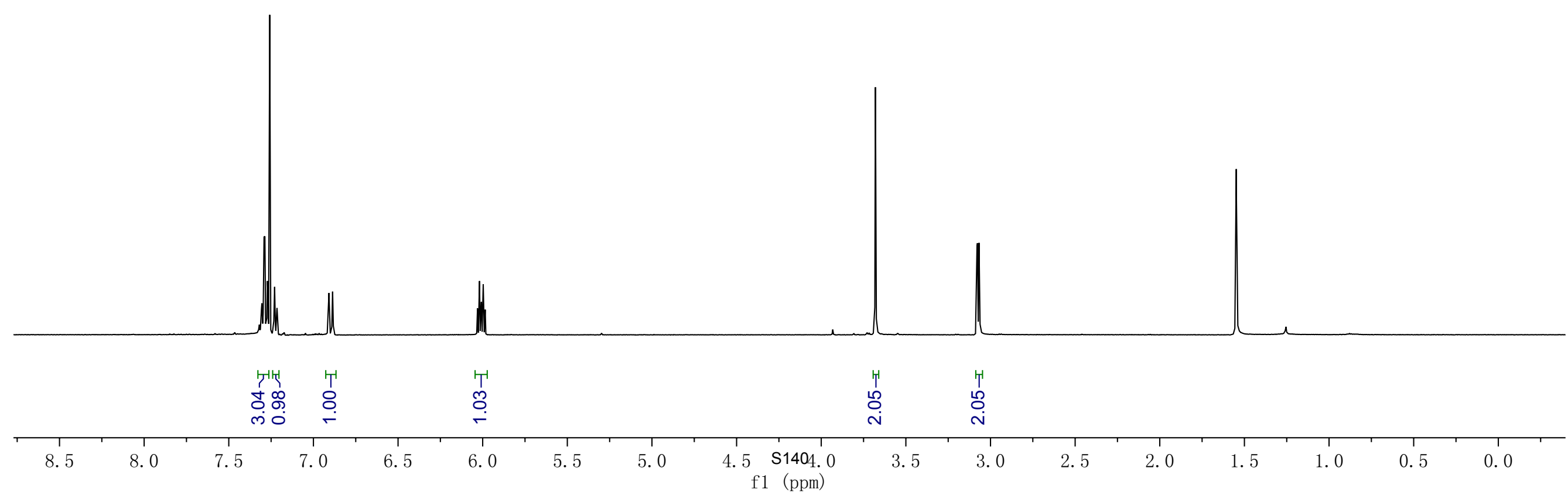




\begin{tabular}{|ll|}
\hline \multicolumn{1}{|c|}{ Parameter } & \multicolumn{1}{c|}{ Value } \\
1 Title & $\mathrm{xz}-1-241 \mathrm{CC}-500$ biaopu \\
2 Solvent & $\mathrm{CDCl3}$ \\
3 Spectrometer Frequency & 125.70 \\
4 Nucleus & $13 \mathrm{C}$ \\
\hline
\end{tabular}

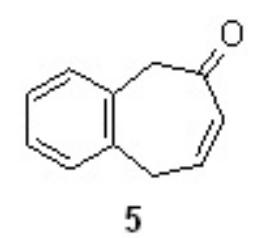

윤ำ 

\section{ำ 8 논}

î́n

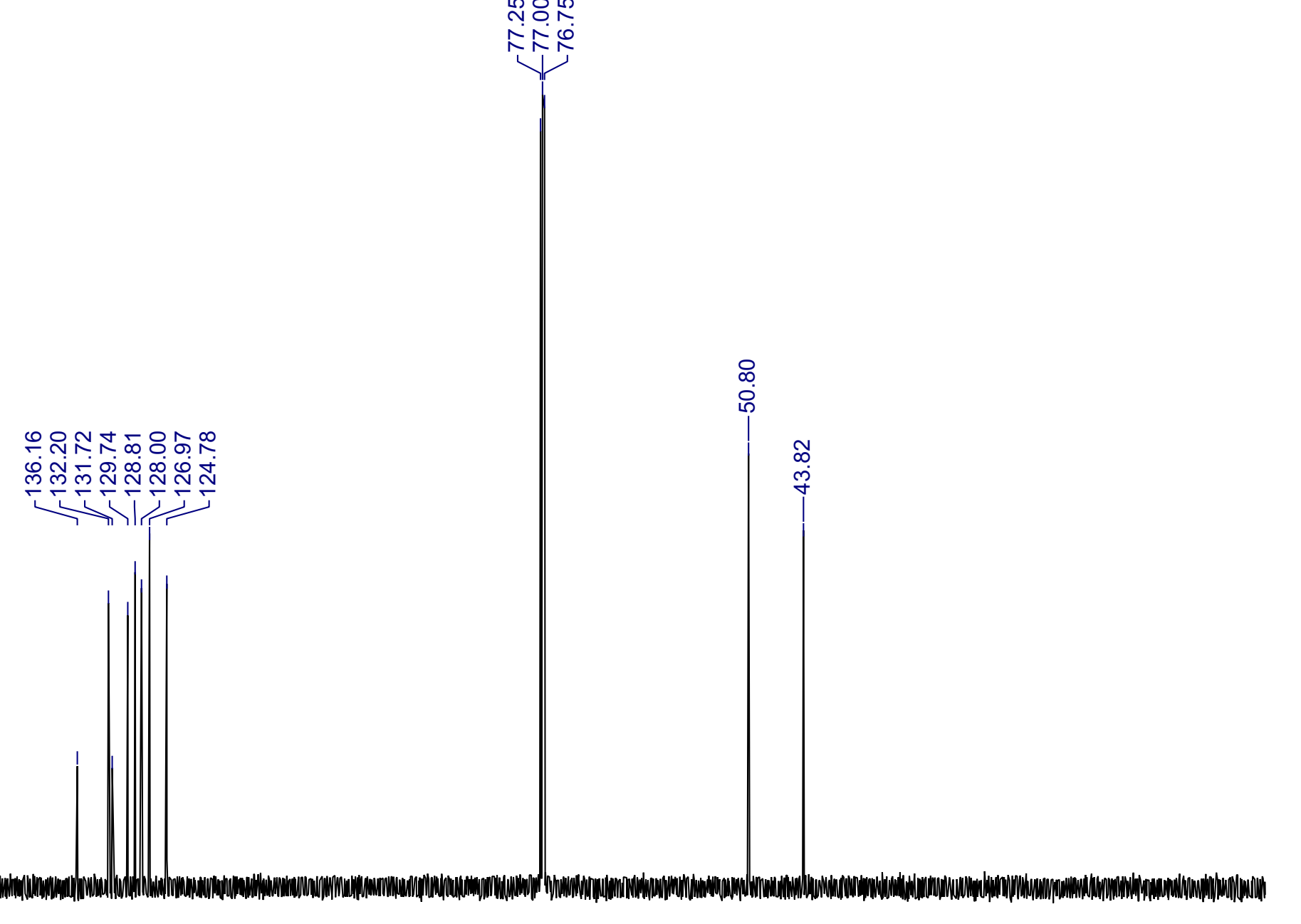

\begin{tabular}{|c|c|c|c|c|c|c|c|c|c|c|c|c|c|c|c|c|c|c|c|c|c|c|}
\hline $\begin{array}{c}1 \\
220\end{array}$ & 210 & 200 & 190 & 180 & 170 & 160 & 150 & 140 & 130 & 120 & $\begin{array}{l}110 \mathrm{~S} 141100 \\
\mathrm{f} 1 \quad(\mathrm{ppm})\end{array}$ & 90 & 80 & $\begin{array}{l}1 \\
70\end{array}$ & 60 & 50 & $\begin{array}{c}1 \\
40\end{array}$ & 30 & 20 & 10 & 0 & -10 \\
\hline
\end{tabular}

